\title{
BENEFITS AND DRAWBACKS OF THE PLACENTA IN LIVE-BEARING FISH
}
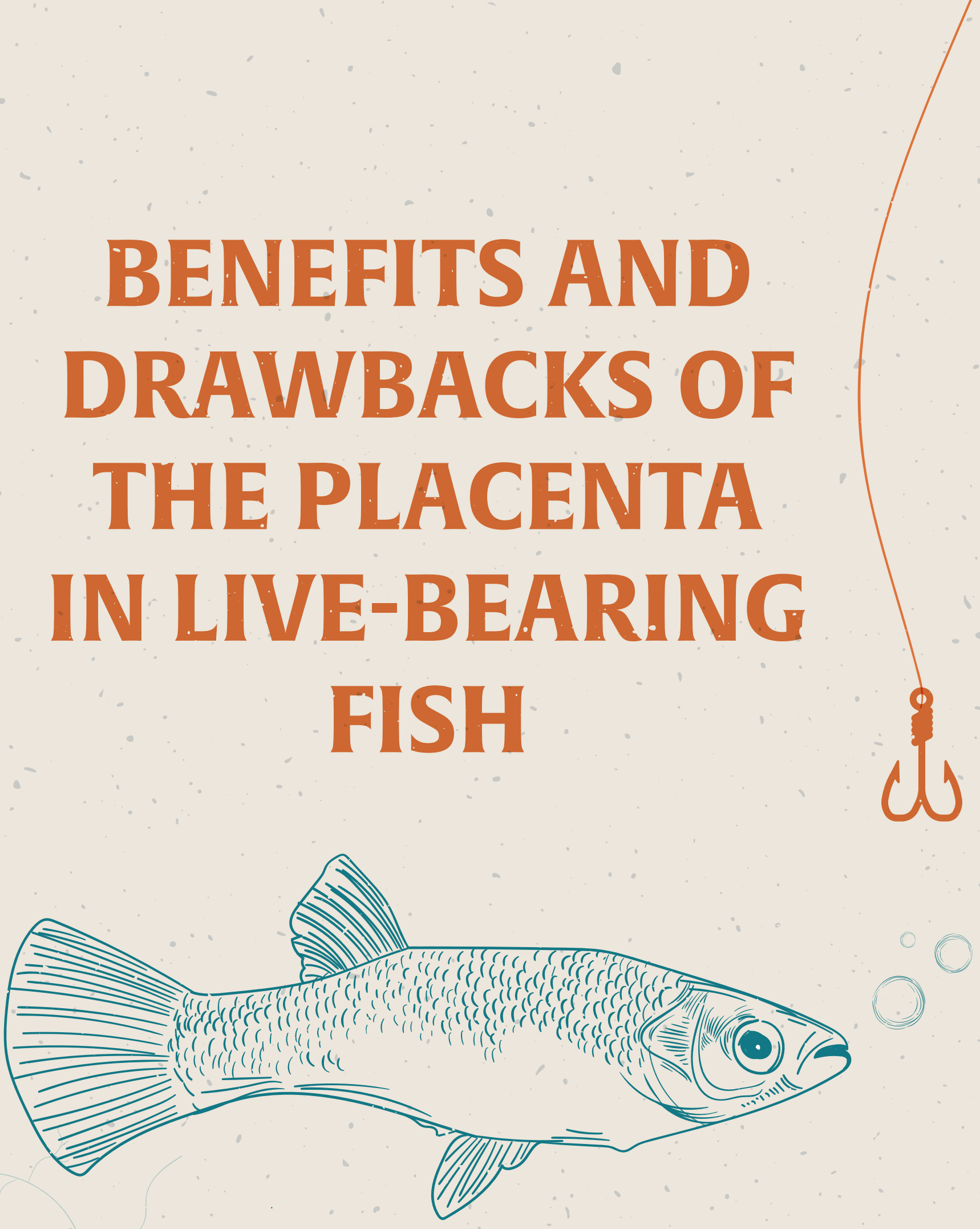

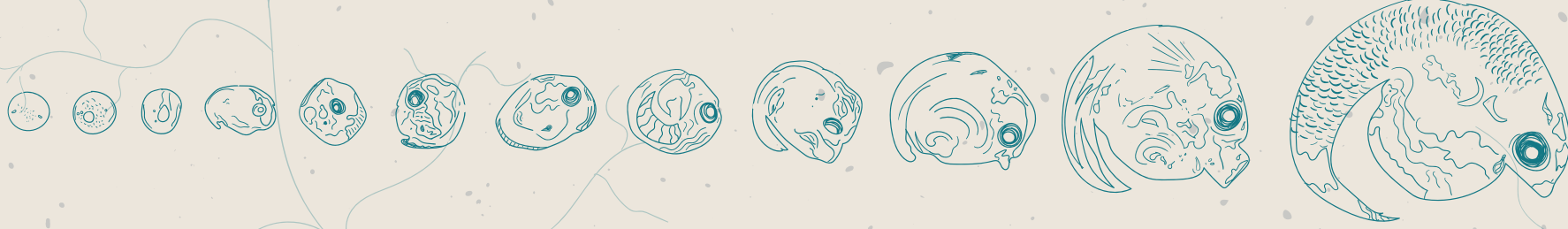





\title{
Propositions
}

1. Placental variation is likely the result of an interaction between phenotypic plasticity acting on offspring mass and evolved differences in egg mass. (this thesis)

2. The intimate link the placenta forms between the mother and fetus poses a risk in environments with low food availability or high parasitism. (this thesis)

3. Multivariate mixed models provide the preferred approach to simultaneously test for multiple hypotheses.

4. Repeated measurements taken over different timescales should be more frequently used to explicitly account for measurement error.

5. The conflict between farmers, hunters, and general public arising from the re-appearance of the wolf in the alps reflects the rapid humaninduced habitat loss during the last century.

6. By forcing researchers to operate in a virtual world, COVID-19 promotes scientific networking, and hence, progress in science.

The propositions belong to the thesis, entitled

\section{Benefits and drawbacks of the placenta in live-bearing fish}

\author{
Andres Hagmayer \\ Wageningen, 26 February 2021
}





\section{Benefits and drawbacks of the placenta in live-bearing fish}




\section{Thesis committee}

\section{Promotor:}

Prof. Dr Johan L. van Leeuwen

Professor of Experimental Zoology

Wageningen University \& Research

\section{Co-promotor:}

Dr Bart J.A. Pollux

Assistant Professor, Experimental Zoology

Wageningen University \& Research

\section{Other members:}

Prof. Dr F. van Langevelde, Wageningen University \& Research

Prof. Dr M.E. Maan, University of Groningen

Dr J.M. Koene, Vrije Universiteit Amsterdam

Prof. Dr C. Ghalambor, Norwegian University of Science \& Technology, Trondheim, Norway

This research was conducted under the auspices of the Graduate School of the Wageningen Institute of Animal Sciences (WIAS) 


\title{
Benefits and drawbacks of the placenta in live-bearing fish
}

\author{
Andres Hagmayer
}

Thesis

submitted in fulfilment of the requirements for the degree of doctor at

Wageningen University

by the authority of the Rector Magnificus

Prof. Dr A.P.J. Mol,

in the presence of the

Thesis Committee appointed by the Academic Board

to be defended in public

on Friday 26 February 2021

at 4 p.m. in the Aula. 
Andres Hagmayer

Benefits and drawbacks of the placenta in live-bearing fish 228 pages.

PhD thesis, Wageningen University, Wageningen, the Netherlands (2021)

With references, with summaries in English and German

ISBN 978-94-6395-627-7

DOI $10.18174 / 535584$ 




\section{Table of contents}

$\begin{array}{lll}\text { Chapter } 1 \text { General introduction } & 9\end{array}$

Chapter 2 Maternal size and body condition predict the amount of postfertilization maternal provisioning in matrotrophic fish

Chapter 3 Predation risk shapes the degree of placentation in natural populations of live-bearing fish

Chapter 4 Differences in ontogenetic and diurnal microhabitat selection by sympatric live-bearing fish species with different reproductive modes

Chapter 5 Parasite infestation influences life history but not boldness behavior in placental live-bearing fish

Chapter 6 Maternal food limitation during pregnancy affects offspring growth and locomotor performance after birth in a matrotrophic live-bearing fish

Chapter 7 General discussion

Declarations

Authorship statements

Ethical statements

Funding

Conflict of interest

Data availability

References

Summaries

Summary

Zusammenfassung

Acknowledgements

Personalia

Curriculum vitae 


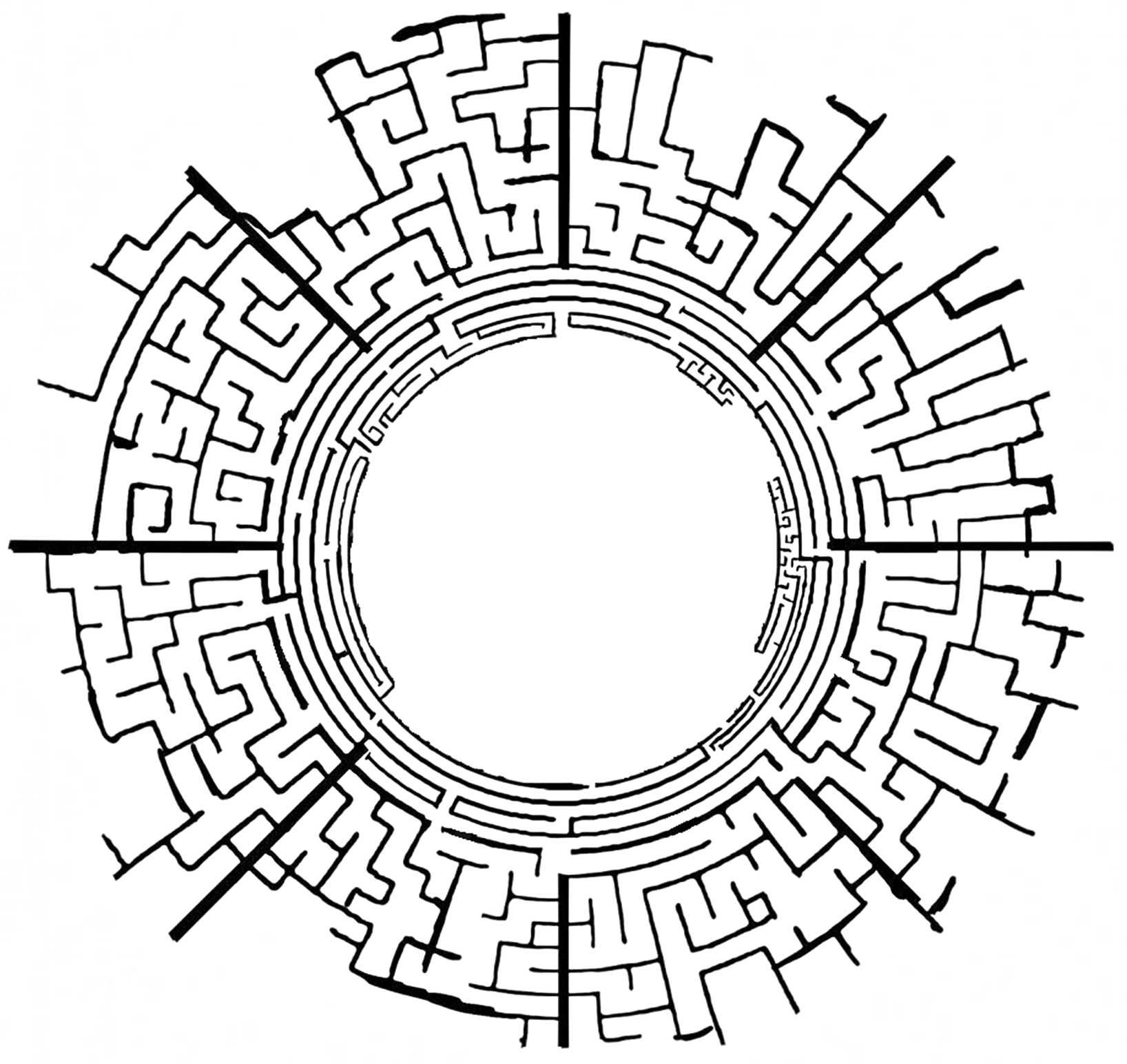




\section{General introduction}

Understanding the origin and elaboration of complex traits is of fundamental interest to scientists and non-scientists alike. Darwin was the first to propose a mechanistic explanation for the evolution of complex traits by suggesting that complexity can evolve by a gradual stepwise process from simple precursors through natural selection (Darwin, 1859). However, finding examples of this stepwise process is challenging, as the intermediate steps in the evolution of a complex trait have often been lost through extinction. The placenta, an intimate fusion of maternal and fetal tissues, is an ideal example of a complex structure that mediates nutrient transfer and physiological interactions between mother and developing offspring (Mossman, 1987). Normal functioning requires a wide range of interdependent factors, all of which seem to be essential for embryo development (Wooding \& Burton, 2008). Placentas have evolved independently throughout the animal kingdom in live-bearing fish (Wourms, 1981), sharks (Hamlett et al., 1993), reptiles (Blackburn, 2015), amphibians (Wake, 2015), mammals (Wooding \& Burton, 2008), and a variety of invertebrates (Ostrovsky et al., 2016). This repeated evolution clearly points to a possible adaptive advantage (Losos et al., 1998), however, the nature of this advantage remains elusive.

In this thesis, I use laboratory experiments and extensive field work in Costa Rica to study different aspects of the phenotype and ecology of live-bearing fish (e.g. life-history, swimming performance, microhabitat selection). Specifically, by drawing on insights from the live-bearing fish family Poeciliidae, I aim to shed light on the obscure origin of the placenta. Furthermore, I examine the impacts of environmental stressors during pregnancy on early life development of offspring, as well as the consequences of acquiring a placenta for a species' ecology.

\subsection{Modes of maternal provisioning in Poeciliidae}

The fish family Poeciliidae contains well known species such as the guppy, swordtails, and mollies that have become model systems in the study of sexual and natural selection (Evans et al., 2011). All species in the family, save one, give live-birth. Strikingly, there is exceptional diversity in the strategy of how to nourish developing embryos, which can be quantified by the Matrotrophy Index (MI), calculated as the ratio of offspring mass 
at birth to egg mass at fertilization (Reznick et al., 2002) (Fig. 1.1a). Most species are lecithotrophs (or 'yolk-feeding'), committing all nutrients required for development during pregnancy to the eggs prior to fertilization (Fig. 1.1b). Embryos subsequently lose dry mass over the course of gestation due to metabolic processes, leading to an MI $<1$. In the remaining species, so-called matrotrophs (i.e. 'mother-feeding'), the resource allocation to offspring takes place during two distinct periods (Wourms, 1981). Part of the nutrients are supplied pre-fertilization during oogenesis and stored as high-energy yolk (Wallace \& Selman, 1981). The rest of the nutrients are transferred post-fertilization to the developing embryos throughout gestation (Wourms, 1981) (Fig. 1.1b). Such species have an MI >1, indicating that embryos gain dry mass during pregnancy. Placentotrophy represents one specific type of matrotrophy, where post-fertilization maternal provisioning is achieved through a follicular placenta, roughly an analog to the mammalian placenta (Pollux et al., 2009).

a

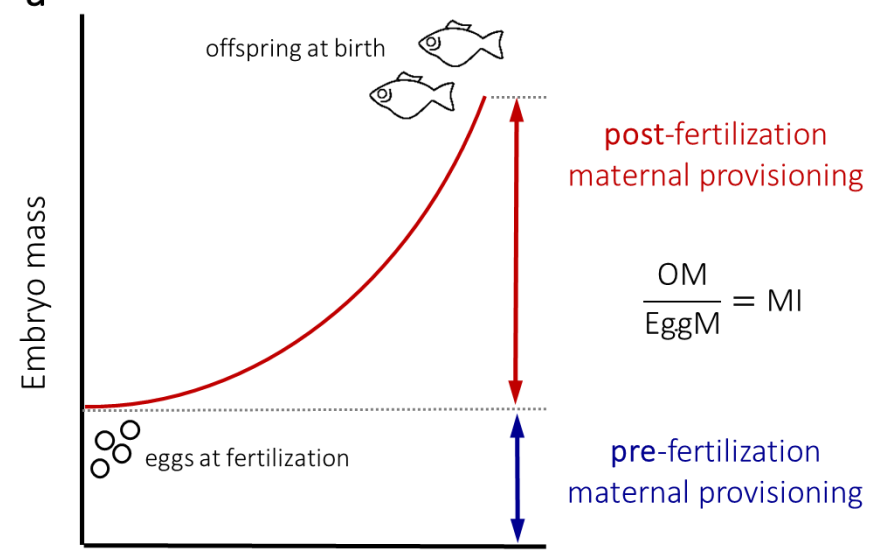

Gestation

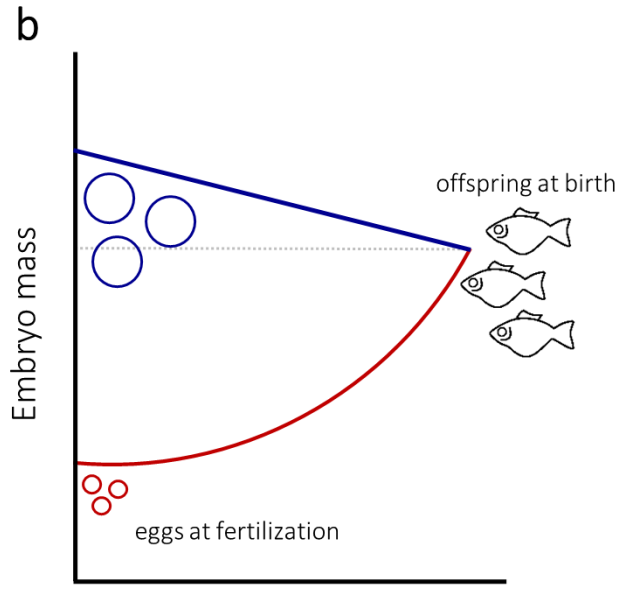

Gestation

Fig. 1.1. a The Matrotrophy Index (MI), calculated as the ratio of offspring mass at birth to egg mass at fertilization, can be used as an unbiased measure of the degree of post-fertilization maternal provisioning, and hence the degree of placentation (Reznick et al., 2002). b Embryo dry mass throughout gestation in live-bearing fish with different modes of reproduction. Lecithotrophic species (blue) produce large eggs at fertilization, providing all maternal resources in the form of yolk prior to fertilization. Embryos subsequently lose dry mass over the course of gestation due to metabolic processes, leading to an $\mathrm{MI}<1$. Matrotrophic species (red) produce small eggs at fertilization with little yolk and provide most resources during embryonic development, leading to an $\mathrm{MI}>1$. The increase in embryo mass is due to post-fertilization maternal provisioning. In placentotrophy, post-fertilization maternal provisioning is achieved through a follicular placenta.

Placentotrophy has evolved independently at least nine times in the family Poeciliidae (Pollux et al., 2009; Furness et al., 2019). Moreover, in this group the MI has been shown to positively correlate with the elaboration of the structures that form the inter- 
face between the mother and offspring, and hence to reflect the complexity of placental morphology (Turner, 1940; Kwan et al., 2015; Olivera-Tlahuel et al., 2019). Overall, these features make poeciliid fish a unique model system for answering questions regarding the causes and consequences of placental evolution (e.g. Pollux et al. 2009, Pollux et al. 2014).

\subsection{Adaptive hypotheses for the evolution of the pla- centa}

To date, two adaptive hypotheses (termed the resource allocation and locomotor cost hypotheses) provide potential explanations for why the placenta evolves. Both hypotheses treat the placenta as a life-history adaptation that evolves in response to ecological selection pressures (Thibault \& Schultz, 1978; Trexler \& DeAngelis, 2003). Furthermore, both hypotheses posit that the ancestral state is for all nutrients to be prepackaged in the form of egg yolk (lecithotrophy) and that the derived state is for nutrients to be supplied to embryos throughout pregnancy via a placenta (placentotrophy). This is presumably achieved via a gradual shift in the timing of nutrient provisioning from pre- to post-fertilization. If true, then the evolution of the placenta should coincide with a reduction in egg mass at fertilization without affecting the size and quality of offspring at birth (Reznick et al., 2002; Trexler \& DeAngelis, 2003; Pollux et al., 2009).

The locomotor cost hypothesis posits that the placenta evolves as an adaptation that reduces a live-bearing female's reproductive allotment (i.e. the proportion of female mass allocated to developing offspring), thus facilitating streamlining and hence locomotor performance, particularly early in pregnancy (Fig. 1.2). The physical and physiological burden of pregnancy negatively affects a female's locomotor performance in a broad range of live-bearing animals (Seigel et al., 1987; Plaut, 2002; Noren et al., 2011; Fleuren et al., 2019). In live-bearing fish, pregnancy causes a less slender body shape (Fleuren et al., 2018), slows the fast-start escape response, and reduces sustained swimming performance (Plaut, 2002; Ghalambor et al., 2004; Quicazan-Rubio et al., 2019). Thus, the production of smaller eggs at fertilization and gradual nutrient allocation to embryos over the course of gestation is predicted to result in less-impaired locomotor performance early in pregnancy without affecting the female's reproductive output (i.e. fecundity). Improved performance is predicted to offer a selective advantage, particularly in high 'performancedemanding' environments (e.g. in high-flow velocity or high-predation localities) due to an enhanced survival probability (Walker et al., 2005; Pollux et al., 2009; Pires et al., 2011) (Fig. 1.2). 


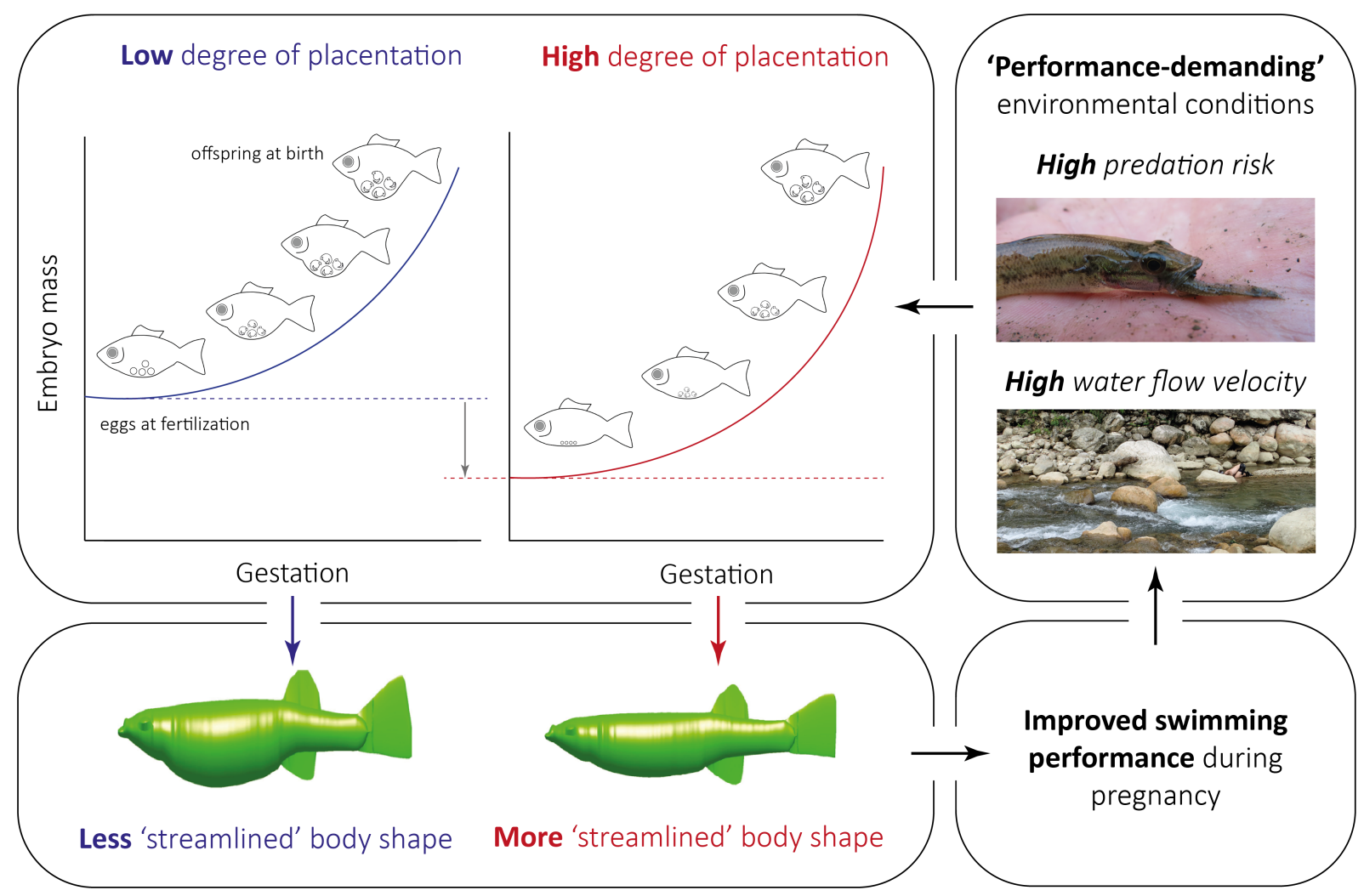

Fig. 1.2. The locomotor costs hypothesis posits that the evolution of the placenta reduces a live-bearing female's reproductive allotment (i.e. the proportion of female mass allocated to developing offspring), thus facilitating streamlining and hence swimming performance, particularly early in pregnancy. This subsequently enhances survival probability in high 'performance-demanding' environments (e.g. in high-flow velocity or high-predation localities) (Pollux et al., 2009).

The resource allocation hypothesis predicts placentas will enhance fecundity in stable, high-resource environments (Trexler \& DeAngelis, 2003). Placental females produce smaller, less expensive eggs, and transfer nutrients to the developing embryos throughout gestation (Wourms, 1981). By reducing the initial cost of eggs, placental females of equal size and body condition have the potential to fertilize more eggs and gestate more embryos. If sufficient nutrients are available to provision all developing embryos, placental provisioning may increase maternal fecundity (Trexler \& DeAngelis, 2003). If food is scarce or fluctuating, females can provision young with stored maternal fat reserves or abort and resorb them and provision only as many as they can fully nourish (Trexler, 1997; Trexler \& DeAngelis, 2003). However, if placental females are unable to abort embryos and recycle the invested energy, the benefits of placentation are greatly reduced. Trexler \& DeAngelis (2003) therefore argued that the ability to selectively abort embryos and recycle this investment, should resource conditions suddenly deteriorate, is a crucial preadaptation for the evolution of placentation. Studies in the Poeciliidae have 
not shown that they are able to do this, suggesting that the conditions under which the placenta might be favored by natural selection are restricted to environments characterized by high and stable resource conditions (Reznick et al., 1996b; Banet \& Reznick, 2008; Banet et al., 2010; Pollux \& Reznick, 2011). Whereas the resource allocation hypothesis has already been the focus of several empirical studies (Banet \& Reznick, 2008; Banet et al., 2010; Pires et al., 2011; Pollux \& Reznick, 2011; Bassar et al., 2014), the locomotor cost hypothesis has not yet been subject to similar systematic investigation.

\subsection{Life-history consequences of the evolution of the pla- centa}

The intimate link the placenta forms between mother and fetus also poses a risk, because maternal exposure to adverse environmental conditions (e.g. malnutrition, parasite infestation) may have unfavorable consequences for fetal development. For instance, manipulations of food availability under laboratory conditions have been demonstrated to impact the pattern of resource allocation to offspring in placental live-bearing fish. Because placental species continuously supply the developing embryos with resources (Wourms, 1981; Pollux et al., 2009), the timing of determining brood size (i.e. number of embryos) and offspring size are decoupled (Reznick et al., 1996b; Pollux \& Reznick, 2011). Whereas brood size is determined prior to fertilization based on current food availability, offspring size may be affected by food availability after fertilization, throughout gestation (Reznick et al., 1996b; Pollux \& Reznick, 2011). Consequently, when resource conditions suddenly deteriorate, placental species may not be able to optimally provision their embryos. Because poeciliid fish may be incapable of selectively aborting embryos in response to adverse food conditions (Reznick et al., 1996b; Banet \& Reznick, 2008; Banet et al., 2010; Pollux \& Reznick, 2011), and maternal body fat might not fully buffer placental females during gestation (Pollux \& Reznick, 2011), sudden resource declines inevitably result in smaller, worse-conditioned offspring at birth (Reznick et al., 1996b; Pollux \& Reznick, 2011). Small offspring size in low food conditions is associated with a lower survival. Therefore, placentotrophy has been argued to be maladaptive in fluctuating resource environments (Reznick et al., 1996b; Trexler \& DeAngelis, 2003; Pollux \& Reznick, 2011). Likewise, maternal parasite infestation can affect fetal growth in two non-mutually exclusive ways: (i) directly, through infestation of the developing fetus by parasites that can cross the placental barrier; and (ii) indirectly, through the modification of maternal physiology or metabolism to such an extent that it interferes with fetal development. However, the consequences of parasite infestation for embryo development, offspring quality, and life-history traits in non-mammalian placental lineages are currently insufficiently understood. 


\subsection{Ecological consequences of the evolution of the pla- centa}

Acquiring a placenta may have consequences for a species' ecology. Placentation is hypothesized to reduce a female's reproductive burden, enhance body streamlining, and improve the locomotor performance, particularly early in pregnancy (Pollux et al., 2009; Pires et al., 2011) (Fig. 1.2). It has therefore been argued that this reproductive adaptation facilitates a life in 'performance-demanding' environments (e.g. high water flow velocity), if the food conditions are sufficiently stable (Pollux et al., 2009). If true, then placental species should be able to utilize higher 'performance-demanding' (micro)habitats compared to closely related non-placental species. However, empirical evidence for the role of placentation in shaping microhabitat selection of live-bearing fish in stream ecosystems is still lacking.

\subsection{Aims and outline of this thesis}

With this project, I attempt to shed light on the causes and consequences of placental evolution by drawing on insights from the live-bearing fish family Poeciliidae. Specifically, I aim to quantify biologically relevant sources of variation in placentation to identify potential selective agents that ultimately drive the evolution of the placenta. Furthermore, I examine the impacts of environmental stressors during pregnancy on early life development of offspring, as well as the consequences of acquiring a placenta for a species' ecology (Fig. 1.3).

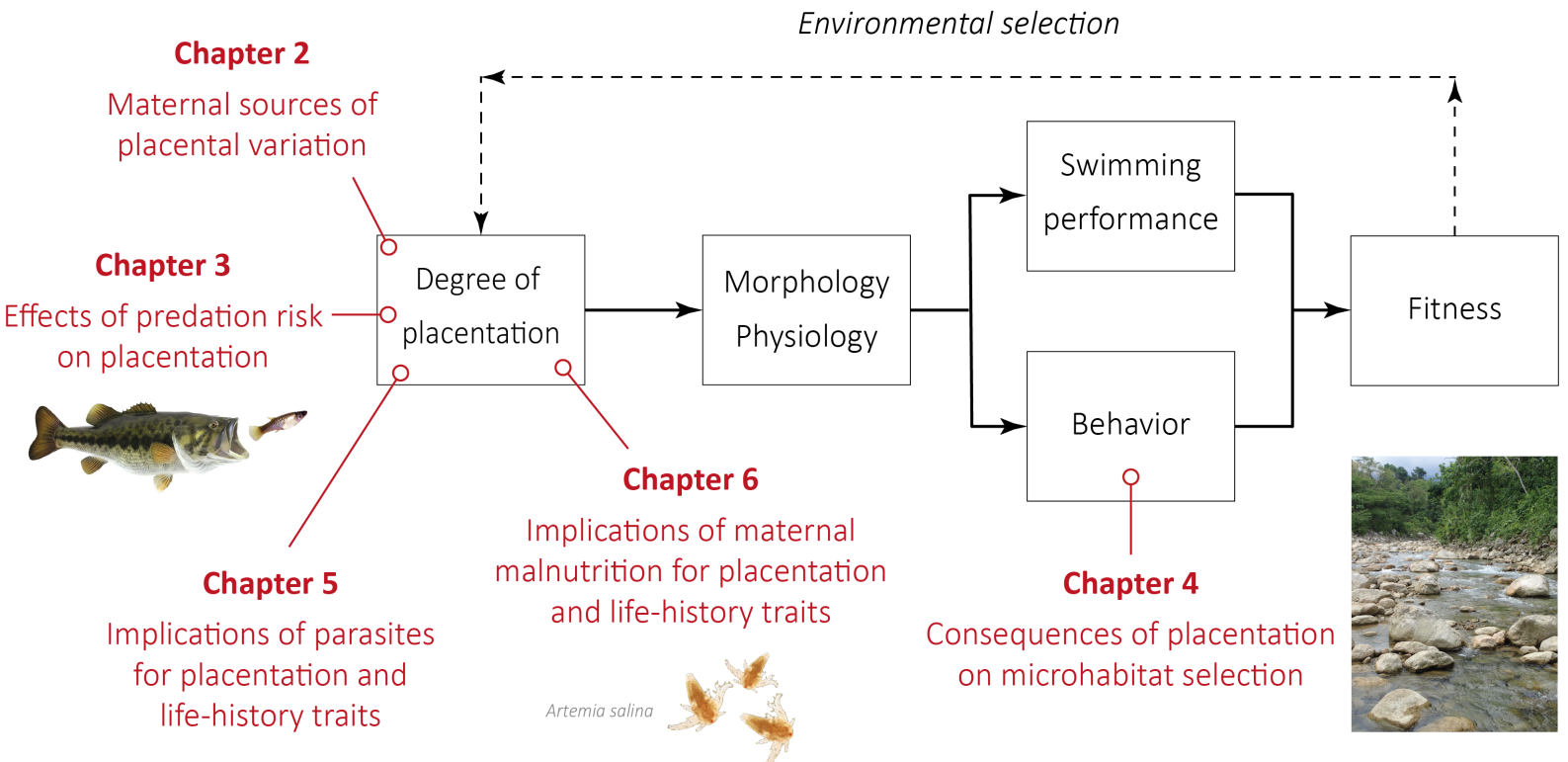


Fig. 1.3. Schematic overview of the thesis chapters. Chapter 2 examines maternal condition-, size- and/or age-related offspring provisioning, and hence maternal sources of placental variation. Chapter 3 tests a key prediction of the locomotor cost hypothesis, which is that placentation evolves in 'performance-demanding' (high predation) environments. Chapter 4 empirically tests the idea that the evolution of the placenta facilitates the utilization of high 'performance-demanding' (micro)habitats (e.g. high-flow velocity) by reducing the reproductive burden of females during pregnancy, and hence, improving locomotor performance. Chapter 5 and 6 examine the potential risks of the intimate link the placenta forms between the mother and fetus when exposed to adverse environmental conditions (maternal parasite infestation and malnutrition). Lastly, in Chapter 7 (not depicted in this picture), the results of the previous chapters will be placed into a wider context with a perspective for future work.

In Chapter 2, we study potential maternal causes and consequences of embryo provisioning during gestation in the placental live-bearing fish species Poeciliopsis retropinna from Costa Rica. Specifically, we examine how maternal traits (i.e. body fat, lean mass, and length) relate to pre- (i.e. allocation to the egg prior to fertilization) and post-fertilization (i.e. allocation to the embryo during pregnancy) maternal provisioning and how this ultimately affects offspring size and body composition at birth. By doing this, we aim to provide new insights into maternal condition-, size- and/or age-related offspring provisioning in placental species, and hence, maternal sources of placental variation.

In Chapter 3, we study natural populations of the placental live-bearing fish species $P$. retropinna in Costa Rica to test a key prediction of the locomotor cost hypothesis, which is that placentation evolves in 'performance-demanding' (high predation) environments. If the placenta is favored under high predation conditions, we should find a higher degree of placentation in high predation populations. Theory further predicts that this higher degree of placentation should be due to a smaller egg size at fertilization without affecting the number, size, or quality of offspring at birth, translating to a lower reproductive burden for females during pregnancy. By studying the ecological drivers that underlie the ongoing evolution of placentas in natural populations, we aim to contribute to a better understanding of the ecologically-driven evolution of complex traits in general.

In Chapter 4, we empirically test the idea that the evolution of the placenta (and superfetation, i.e. the ability to carry several broods at different developmental stages) facilitates a life in 'performance-demanding' (e.g. high-flow velocity) environments by reducing the reproductive burden of females during pregnancy, and hence, improving locomotor performance. If true, we predict placental species and/or species with superfetation to inhabit higher 'performance-demanding' (micro)habitats compared to closely related non-placental species or species without superfetation. For this, we use underwater visual census to study diurnal and ontogenetic microhabitat selection by five sympatric livebearing fish species (family Poeciliidae) in Costa Rica that differ in the absence/presence of placentation and superfetation. By comparing microhabitat use of these five sympatric live-bearing fish species, we aim to provide new insights into the potential effects 
of reproductive adaptations on microhabitat selection and local diurnal and ontogenetic migration.

In Chapter 5, we examine the consequences of a trematode infestation (black spot disease, BSD) in shaping life-history and behavior (boldness trials in the field) in the placental live-bearing fish species $P$. retropinna from Costa Rica. We propose that the intimate link the placenta forms between the mother and fetus poses a risk, because parasite infestation may have unfavorable consequences for fetal development. For example, maternal parasite infestation can affect fetal growth in two non-mutually exclusive ways: (i) directly, through infestation of the developing fetus by parasites that can cross the placental barrier; and (ii) indirectly, through the modification of maternal physiology or metabolism to such an extent that it interferes with fetal development. By examining the potential costs of a trematode infestation in placental live-bearing fish, we aim to contribute to a better understanding of the importance of host-parasite interactions in shaping life-histories and behavior in placental animals.

In Chapter 6, we conduct a 7-week laboratory experiment to study to what extent maternal food limitation during pregnancy affects offspring size and quality (i.e. body fat) at birth, as well as growth and locomotor performance of offspring after birth in the placental live-bearing fish species Phalloptychus januarius. We predict that having a placenta might be a maladaptive strategy in environments where food is temporarily scarce, because sudden reductions in maternal food availability are likely to cause the production of smaller offspring at birth that perform worse during early life. By studying the impacts of environmental stressors during pregnancy on early life development of offspring, we aim to shed light on the potential burden of placentation under adverse environmental conditions.

In the general discussion (Chapter 7), I put the findings of my PhD research into a wider context. First, I restate the different hypotheses for the evolution of the placenta together with the currently available evidence for each. Second, because superfetation is commonly found in Poeciliidae and thought to co-evolve with the evolution of the placenta, I develop an R-package to simulate and graphically illustrate the potential advantages of having superfetation, as well as both, superfetation and placentation. Third, I discuss the potential disadvantages of having a placenta in response to various adverse environmental conditions. Finally, I conclude with an outlook on future research by addressing possible steps and challenges to deepen and expand the knowledge on the causes and consequences of placental evolution. 



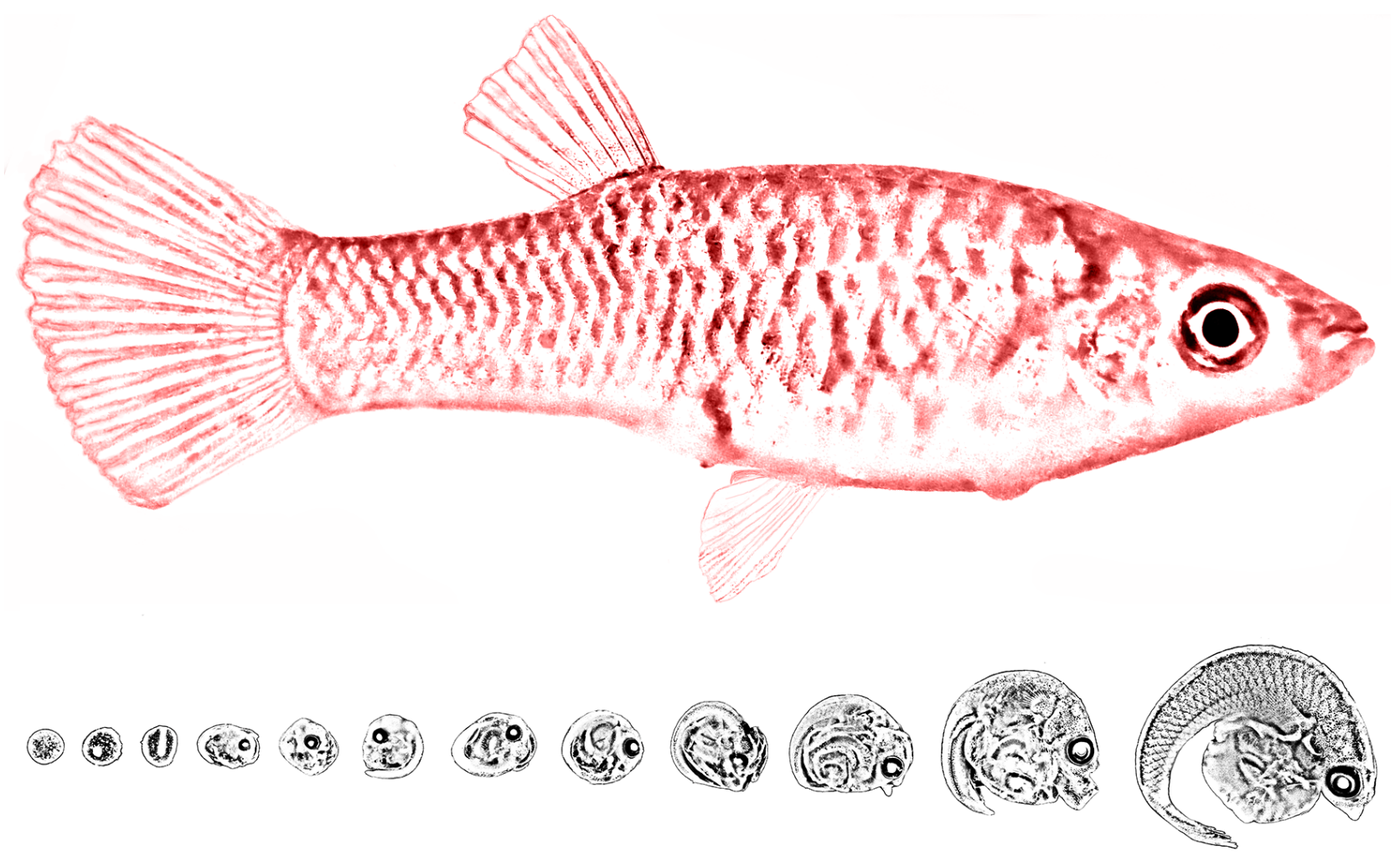




\title{
2 Maternal size and body condition predict the amount of post-fertilization maternal provi- sioning in matrotrophic fish
}

\author{
Andres Hagmayer ${ }^{1}$, Andrew I. Furness ${ }^{2,3}$, David N. Reznick ${ }^{4}$ \\ \& Bart J.A. Pollux ${ }^{1}$
}

1 Department of Animal Sciences, Wageningen University, 6708 WD Wageningen, The Netherlands

2 Department of Ecology and Evolutionary Biology, University of California, CA 92697 Irvine, USA

${ }^{3}$ Department of Biological and Marine Sciences, University of Hull, HU6 7RX Hull, UK

4 Department of Biology, University of California, CA 92521 Riverside, USA

This chapter has been published as: Hagmayer, A., et al. (2018). Maternal size and body condition predict the amount of post-fertilization maternal provisioning in matrotrophic fish. Ecology and Evolution, 8(24):12386-12396. https://doi.org/10.1002/ ece3. 4542 


\section{Abstract}

Maternal effects often provide a mechanism for adaptive transgenerational phenotypic plasticity. The maternal phenotype can profoundly influence the potential for such environmentally-induced adjustments of the offspring phenotype, causing correlations between offspring and maternal traits. Here we study potential effects of the maternal phenotype on offspring provisioning prior to and during gestation in the matrotrophic live-bearing fish species Poeciliopsis retropinna. Specifically, we examine how maternal traits such as body fat, lean mass, and length relate to pre- (i.e. allocation to the egg prior to fertilization) and post-fertilization (i.e. allocation to the embryo during pregnancy) maternal provisioning and how this ultimately affects offspring size and body composition at birth. We show that pre- and post-fertilization maternal provisioning is associated with maternal length and body fat, but not with maternal lean mass. Maternal length is proportionally associated with egg mass at fertilization and offspring mass at birth, notably without changing the ratio of pre- to post-fertilization maternal provisioning. This ratio, referred to as the matrotrophy index (MI), is often used to quantify the level of matrotrophy. By contrast, the proportion of maternal body fat is positively associated with post-fertilization, but not pre-fertilization, maternal provisioning and consequently is strongly positively correlated with the MI. We furthermore found that the composition of embryos changes throughout pregnancy. Females invest first in embryo lean mass, then allocate fat reserves to embryos very late in pregnancy. We argue that this delay in fat allocation may be adaptive, because it delays an unnecessary high reproductive burden to the mother during earlier stages of pregnancy, potentially leading to a more slender body shape and improved locomotor performance. In conclusion, our study suggests that (i) offspring size at birth is a plastic trait that is predicted by both maternal length and body fat, and (ii) the MI is a plastic trait that is predicted solely by the proportion of maternal body fat. It herewith provides new insights into the potential maternal causes and consequences of embryo provisioning during pregnancy in matrotrophic live-bearing species.

Keywords: live-bearing, matrotrophy, placenta, placentotrophy, Poeciliidae, maternal effect, superfetation, viviparity

\subsection{Introduction}

Maternal effects represent the influence of the mother's phenotype on the offspring phenotype independently of the female's genetic contribution to her offspring (Mousseau \& Fox, 1998a). In species where a female's environment is a reliable predictor of the environmental conditions that her future offspring are likely to experience, females may evolve the ability to adjust offspring phenotype in ways that best prepare them for life in their future environment. Here, maternal effects will provide a mechanism for adaptive 
transgenerational phenotypic plasticity (Mousseau \& Fox, 1998b).

Maternal effects often entail complex, multiple-interacting trade-offs. Maternal nutrient provisioning to offspring, for instance, can have profound implications for offspring size at birth and hence offspring survival (Mousseau \& Fox, 1998a). By changing the offspring phenotype, maternal provisioning may also directly affect maternal fitness, as offspring size is commonly constrained by offspring number (Stearns, 1992). The optimal offspring size is given by the offspring size-performance relationship that is determined by the environment experienced by the offspring (Kaplan, 1992; Fox \& Czesak, 2000; Marshall $\&$ Keough, 2008b). Thus, selection is expected to favor the production of differently sized offspring in different environments (Mousseau \& Fox, 1998b). Generally, selection favors the production of larger offspring in relatively harsher environments (Sibly \& Calow, 1983; Marshall et al., 2010). For instance, strong intra- and interspecific competition typically favors larger offspring (Parker \& Begon, 1986), as larger offspring have been shown to be better competitors for food (Bashey, 2008; Leips et al., 2013). In addition, a variety of other environmental factors, including positive size-dependent predation, low temperature, salinity or food availability can induce adaptive plasticity leading to an increase in offspring size (Kaplan, 1992; Marshall \& Keough, 2008a; Jørgensen et al., 2011).

It has also been argued that the maternal phenotype can significantly influence the potential for environmentally-induced adjustments of the offspring phenotype (Mousseau \& Fox, 1998b; Marshall \& Keough, 2008a). This causes correlations between offspring size and maternal traits such as body condition, size and age across a wide range of taxa (Stearns, 1992). In general, better-conditioned, larger and older mothers are often seen to produce larger offspring (Roff, 1992; Berkeley et al., 2004; Marshall \& Keough, 2008a; Marshall et al., 2010). Moreover, large mothers typically produce more offspring than small mothers (Calder, 1984). A high rate of offspring production may lead to densitydependent sibling competition. Under this condition mothers gain fitness benefits by producing larger, competitive offspring (Parker \& Begon, 1986; Leips et al., 2013). In live-bearers, the survival of the offspring to birth depends on the survival of the mother. If the mother's survival increases with her body size, a theoretical model also predicts a positive mother-offspring size relationship (Jørgensen et al., 2011). Alternatively, size or age-related differential maternal provisioning might be driven by morphological and physiological constraints that limit the maximal offspring size that a female can produce, rather than being caused by response to selection (Congdon \& Gibbons, 1987; Fox \& Czesak, 2000). Thus, maternal phenotype can have important effects, either directly or indirectly, on maternal provisioning, making condition-, size- or age-related differential maternal provisioning an important potential cause of variation in offspring size and fitness.

Although mother-offspring size relationships have been shown to be common in a wide range of taxa (Stearns, 1992), little is known about the influence of maternal condition, size and age on embryo provisioning in matrotrophic live-bearing species. Instead of al- 
locating all resources to the eggs prior to fertilization (i.e. lecithotrophy), matrotrophic species transfer their nutrients to the developing embryos throughout gestation (Wourms, 1981). Since they continuously supply their developing embryos with resources (Wourms, 1981; Pollux et al., 2009), the timing of determining brood size and offspring size can be decoupled (Reznick et al., 1996b; Pollux \& Reznick, 2011). Whereas brood size is determined prior to fertilization based on prior food availability, offspring size is determined after fertilization based on food availability throughout gestation (Reznick et al., 1996b; Pollux \& Reznick, 2011). Matrotrophy, therefore, has been suggested to be a maladaptive strategy in environments where food is scarce or fluctuating because low food availability causes the production of smaller offspring at a time when being large at birth is favored (Reznick et al., 1996b; Trexler \& DeAngelis, 2003, 2010; Pollux \& Reznick, 2011). It has further been argued that matrotrophic species would be better buffered against fluctuating food availability, if they had the ability to diminish brood size via abortion and resorb the invested energy and/or the ability to store large quantities of fat reserves that prevent females from undernourishing their embryos (Trexler, 1997; Trexler \& DeAngelis, 2003). Although there is no evidence for embryo abortion due to low food availability in matrotrophic species of the family Poeciliidae, they do sacrifice their fat reserves to sustain their developing embryos under such conditions (Reznick et al., 1996b; Banet \& Reznick, 2008; Banet et al., 2010; Pollux \& Reznick, 2011). Therefore, maternal size and fat reserves are of evolutionary relevance, especially under adverse food conditions.

Here, we study potential maternal causes and consequences of embryo provisioning during gestation in the matrotrophic live-bearing fish species Poeciliopsis retropinna (family Poeciliidae; Regan, 1908; Fig. 2.1). Specifically, we quantify the relationship of maternal traits (i.e. the proportion of maternal body fat, lean mass, and standard length) with: (i) embryo size and body composition during pregnancy, (ii) offspring size and body composition at birth, (iii) the matrotrophy index (MI), which is a measure of post-fertilization maternal provisioning (Reznick et al., 2002; Pollux et al., 2014), and (iv) fecundity. Body fat is believed to be a good indicator of fish condition (Leips et al., 2013) and standard length has been shown to be a good proxy for age (Reznick et al., 1996a). Poeciliopsis retropinna has superfetation, or the ability to carry several broods at various developmental stages (Turner, 1937), making it a good study system to obtain reliable estimates of embryo mass throughout gestation. We first quantify phenotypic variation in embryo traits (i.e. dry mass, body fat, and lean mass) as a function of developmental stage (Haynes, 1995). The number of embryos is used here as a surrogate measure for maternal fecundity. We then correlate this variation to maternal traits (i.e. the proportion of maternal body fat, lean mass, and standard length) to detect maternal condition-, size- and/or age-related offspring provisioning. Furthermore, we evaluate the consequences for the MI and fecundity. Finally, we discuss our results in light of the evolution of matrotrophy in live-bearing animals. 
Fig. 2.1. Female Poeciliopsis retropinna (family Poeciliidae). Photo: Andrew Furness.

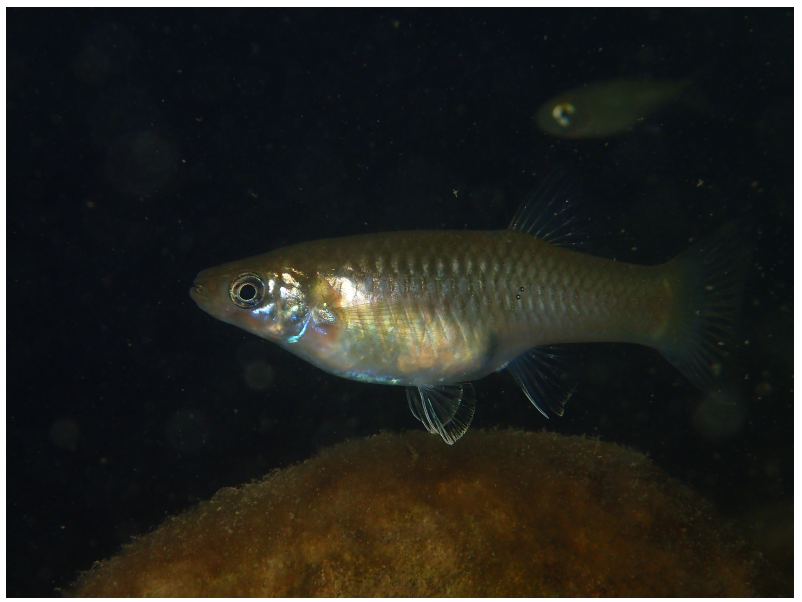

\subsection{Material and methods}

\subsubsection{Study species and collection sites}

Poeciliopsis retropinna, a live-bearing fish species in the family Poeciliidae, reaches a maximum standard length of approximately $80 \mathrm{~mm}$. This species is found in freshwater streams of all current velocities from sea level to $940 \mathrm{~m}$ elevation in Costa Rica and Panama (Bussing, 2002). During gestation, P. retropinna females transfer nutrients to developing embryos via a placenta-like structure (i.e. they exhibit matrotrophy) (Pollux et al., 2009). The degree of post-fertilization maternal provisioning in this species is extensive, with offspring increasing in dry mass more than 100-fold during gestation (MI = 117) (Reznick et al., 2002).

During March 2013, P. retropinna were collected at five different locations in the Rio Terraba drainage in the province of Puntarenas, Costa Rica (Table S.2.1). At each location, 10-30 adult females were collected using seine and cast nets, euthanized with an overdose of MS-222 (Sigma-Aldrich Co., St. Louis, Missouri, USA), and preserved in $5 \%$ formaldehyde (Fisher Scientific, Fair Lawn, New Jersey, USA). The fish samples were transported to the Reznick lab (University of California Riverside, USA) for anatomical dissections.

\subsubsection{Laboratory measurements}

The standard length of preserved specimens was measured to the nearest mm from the tip of the upper jaw to the outer margin of the hypural plate, using a caliper. Female dry mass was measured to the nearest $0.01 \mathrm{mg}$ on a Mettler Toledo AE163 Microbalance (Mettler Instruments Corp., Hightstown, New Jersey, USA) after removing the ovary and air-drying the female overnight at $60^{\circ} \mathrm{C}$ in a drying oven. Only four of the five sampled populations contained pregnant females (Table S.2.1), therefore all subsequent anatomical and statistical analyses were carried out only with the females from these four populations. Female lean mass was measured by extracting the fat twice with anhydrous 
diethyl ether (Fisher Scientific) to remove triglycerides, and by subsequently air-drying and re-weighting the female (see above). The proportion of maternal body fat was then calculated by subtracting maternal lean mass from maternal dry mass divided by maternal dry mass. The ovaries were dissected to count the number of embryos (i.e. fecundity), and to determine the developmental stage and average mass of the embryos for a given brood. Since the embryos are counted across all broods, fecundity reflects a combination between the effects of brood size and superfetation. The developmental stages are based on morphological criteria described in Haynes (1995) and range from 0 (eggs at fertilization, no development) to 45 (fully developed embryos), with stage 50 representing newborn offspring (Haynes, 1995; Reznick et al., 2002). The embryo dry mass for a given brood was calculated as the dry mass of the brood, measured to the nearest $0.01 \mathrm{mg}$ after airdrying overnight at $60^{\circ} \mathrm{C}$ (see above), divided by the number of embryos in the brood (Pollux \& Reznick, 2011). The embryo lean mass was then measured by extracting the fat twice with anhydrous diethyl ether then air-drying and re-weighting the brood, and by dividing by the number of embryos. The proportion of embryo body fat was calculated as described above. Fecundity was calculated by excluding stage 0 embryos (since it was difficult to assess if they were all fertilized). A detailed explanation of the measured maternal and embryo traits is given in Table 2.1.

Table 2.1. Summary of maternal and embryo traits.

\begin{tabular}{|c|c|}
\hline \multicolumn{2}{|r|}{ Maternal traits } \\
\hline Standard length & $\begin{array}{l}\text { Length from the tip of the upper jaw to the outer margin of the hypural } \\
\text { plate; used as a proxy for age }\end{array}$ \\
\hline Dry mass & Dry mass after removing ovary \\
\hline Lean mass & Dry mass after removing ovary and triglycerides \\
\hline Body fat & Maternal lean mass subtracted from maternal dry mass \\
\hline Proportion body fat & $\begin{array}{l}\text { Maternal body fat divided by maternal dry mass; used as a proxy for body } \\
\text { condition (i.e. well-being or fitness) }\end{array}$ \\
\hline Fecundity & Number of embryos carried by a female counted across all broods \\
\hline \multicolumn{2}{|r|}{ Embryo traits } \\
\hline Dry mass & Brood dry mass divided by the number of embryos in the brood \\
\hline Lean mass & $\begin{array}{l}\text { Brood dry mass after removing triglycerides divided by the number of } \\
\text { embryos in the brood }\end{array}$ \\
\hline Body fat & Embryo lean mass subtracted from embryo dry mass \\
\hline Proportion body fat & $\begin{array}{l}\text { Embryo body fat divided by embryo dry mass; used as a proxy for body } \\
\text { condition }\end{array}$ \\
\hline Developmental stage & $\begin{array}{l}\text { Based on morphological criteria that range from } 0 \text { (eggs at fertilization, no } \\
\text { development) to } 45 \text { (fully developed embryos), with stage } 50 \text { representing } \\
\text { newborn offspring }\end{array}$ \\
\hline
\end{tabular}




\subsubsection{Statistical analysis}

To identify potential condition-, size- and/or age-related effects on embryo provisioning, a series of linear mixed effects models were fitted by Maximum Likelihood. The best model was selected on the basis of Akaike's information criterion adjusted for small sample sizes (AICc) (Burnham \& Anderson, 2002). Ln-transformed embryo dry mass was the response variable. Fixed effects in the full model included the proportion of maternal body fat, lean mass, standard length, and the interaction between each of these variables and the developmental stage of the embryos. Mother identity was fitted as random intercept to correct for pseudo-replication and for between-female variation in maternal provisioning that is not accounted by the fixed effects. Population identity was fitted as an additional random intercept accounting for spatial non-independence of observations.

A significant interaction effect between the proportion of maternal body fat, lean mass, or standard length and embryo developmental stage, implies that the maternal effect is not constant during pregnancy and depends on the developmental stage of the embryos. Consequently, a change in the maternal trait will lead to a change in the matrotrophy index (MI), defined as the estimated dry mass of the offspring at birth (stage 45; derived from the model equation) divided by the estimated dry mass of eggs at fertilization (stage 0) (Reznick et al., 2002; Pollux et al., 2014). A non-significant interaction effect, by contrast, implies that the maternal trait is proportionally associated with egg mass at fertilization and offspring mass at birth without changing the MI. In case of a significant interaction term, the relationship between the corresponding maternal trait and egg mass at fertilization is given by the maternal main effect, since main effects are estimated where all other predictors are zero (i.e. stage 0; eggs at fertilization) (Schielzeth, 2010). The relationship between the maternal trait and offspring mass at birth, by contrast, is given by the maternal main effect after subtracting the developmental stage at birth $\left(s_{\text {birth }}\right)$ from the actual developmental stage of the $j$ th brood in the $i$ th mother $\left(s_{i, j}\right)$ in a second model (i.e. $s_{i, j}^{*}=s_{i, j}-s_{\text {birth }}$ ).

To illustrate the association of the maternal traits with embryo body composition (i.e. body fat and lean mass) during gestation, we fit embryo body fat, embryo lean mass, and the proportion of embryo body fat as a function of the same fixed effect structure found to best explain embryo dry mass according to the AICc. Consequently, all the model structures are uniform, which facilitates parameter comparisons and interpretations. Mother and population identity were fitted as random intercepts to correct for pseudo-replication, between-female and -population variation in maternal provisioning that is not accounted by the fixed effects. In case of a significant interaction term, the relationship between the corresponding maternal trait and offspring mass at birth is again quantified by subtracting the developmental stage at birth from the actual developmental stage of a given brood.

We tested the association of the maternal traits with fecundity by fitting a generalized linear mixed effects model by Maximum Likelihood and a log link for the Poisson-distributed 
response. The fixed effects included the maternal traits found to predict embryo dry mass. Population identity was included as a random effect as was the latest developmental stage of embryos (to account for females early in the reproductive cycle).

To optimize normality and homoscedasticity of the model residuals, absolute embryo weights (i.e. dry mass, lean mass, and body fat) were ln-transformed, the proportion of maternal and embryo body fat was arcsin square-root transformed, and developmental stage was transformed to the square-root of its third power. To compare the strength of the relationship with individual maternal traits, the regression coefficients $(\beta)$ were additionally standardized by multiplying with the phenotypic standard deviation of the maternal trait and by dividing by the phenotypic standard deviation of the response variable $\left(\beta^{\prime}\right)$ (Schielzeth, 2010). All the analyses were carried out in $\mathrm{R}$ v 3.1.3 ( $\mathrm{R}$ Core Team, 2020): mixed models were fitted using the lme4 package (Bates et al., 2015), and significance tests for the fixed effects were performed with lmerTest (Kuznetsova et al., 2016).

\subsection{Results}

\subsubsection{Maternal effects on embryo dry mass}

Variation in embryo dry mass throughout development is best explained by the proportion of maternal body fat, standard length, developmental stage of the embryo, and an interaction between the proportion of maternal body fat and the developmental stage of the embryo (Table S.2.2, Online Table S3). Maternal lean mass and the interaction between both maternal lean mass and standard length with developmental stage were excluded as predictors in the final model (Table S.2.2). Embryo dry mass (mg) significantly increases throughout development, especially during late developmental stages $\left(t_{59.442}=5.638\right.$, $P<0.001$; Online Table S3; Fig. 2.2). An increase in maternal standard length (mm) is associated with a proportional increase in both egg dry mass (mg) at fertilization and offspring dry mass $(\mathrm{mg})$ at birth $\left(t_{30.025}=3.214, P=0.003\right.$; Online Table S3) and, hence, the MI does not change (Fig. 2.3a). By contrast, an increase in the proportion of maternal body fat is associated with a significant increase in offspring dry mass $(\mathrm{mg})$ at birth $\left(t_{74.654}=5.369, P<0.001\right.$; Online Table S3.b), but not with egg dry mass $(\mathrm{mg})$ at fertilization $\left(t_{54.291}=-0.343, P=0.733\right.$; Online Table S3.a). An increase in the proportion of maternal body fat thus predicts a significantly higher MI (Fig. 2.3b). 
Fig. 2.2. Embryo dry mass, lean mass and body fat as a function of developmental stage of embryos $( \pm 95 \% \mathrm{Cl})$ predicted for a female of average standard length (i.e. $\left.\bar{y}_{\text {standard length }}=67 \mathrm{~mm}\right)$ and average proportion of maternal body fat (i.e. $\bar{y}_{\text {body fat }}=$ $0.20)$. This prediction is based on the model parameters described in Online Table S3-S5.

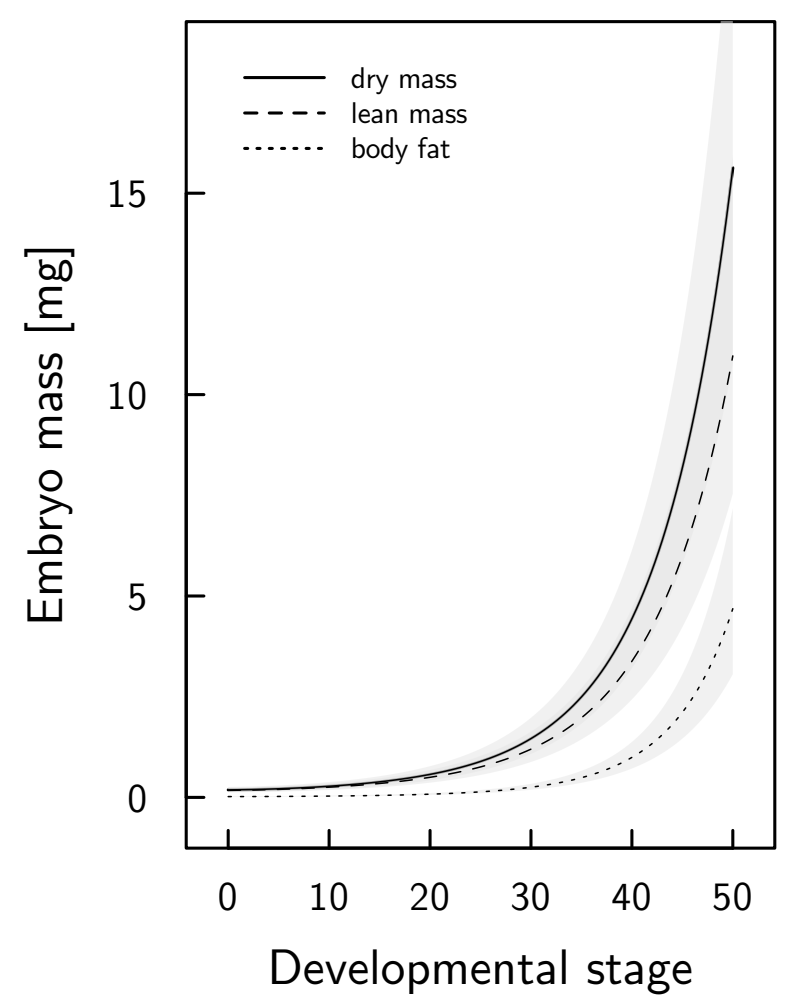

\subsubsection{Maternal effects on embryo lean mass}

Embryo lean mass (mg) significantly increases throughout development, especially late in pregnancy $\left(t_{59.460}=5.776, P<0.001 ;\right.$ Online Table S4; Fig. 2.2$)$. In addition, embryo lean mass increased as a function of increased maternal body fat and standard length (Online Table S4). Maternal standard length $(\mathrm{mm})$ is proportionally related to egg lean mass at fertilization and offspring lean mass at birth $\left(t_{30.608}=3.379, P=0.002\right.$; Online Table S4). The proportion of maternal body fat is positively associated with offspring lean mass $(\mathrm{mg})$ at birth $\left(t_{73.671}=5.046, P<0.001\right.$; Online Table S4.b), but is not significantly correlated to egg lean mass $(\mathrm{mg})$ at fertilization $\left(t_{54.965}=0.066, P=0.948\right.$; Online Table S4.a).

\subsubsection{Maternal effects on absolute amount of embryo body fat}

Embryo body fat $(\mathrm{mg})$ significantly increases during development, but only very late in pregnancy $\left(t_{56.922}=5.499, P<0.001\right.$; Online Table S5; Fig. 2.2). Maternal standard length $(\mathrm{mm})$ is not significantly related to the amount of embryo body fat $(\mathrm{mg})\left(t_{32.052}=\right.$ 0.591, $P=0.559$; Online Table S5). The proportion of maternal body fat, on the other hand, is significantly associated with the amount of body fat (mg) in the offspring at birth $\left(t_{73.273}=4.373, P<0.001\right.$; Online Table S5.b), yet does not correlate with the quantity of fat $(\mathrm{mg})$ in the egg at fertilization $\left(t_{56.605}=0.551, P=0.584\right.$; Online Table S5.a). 

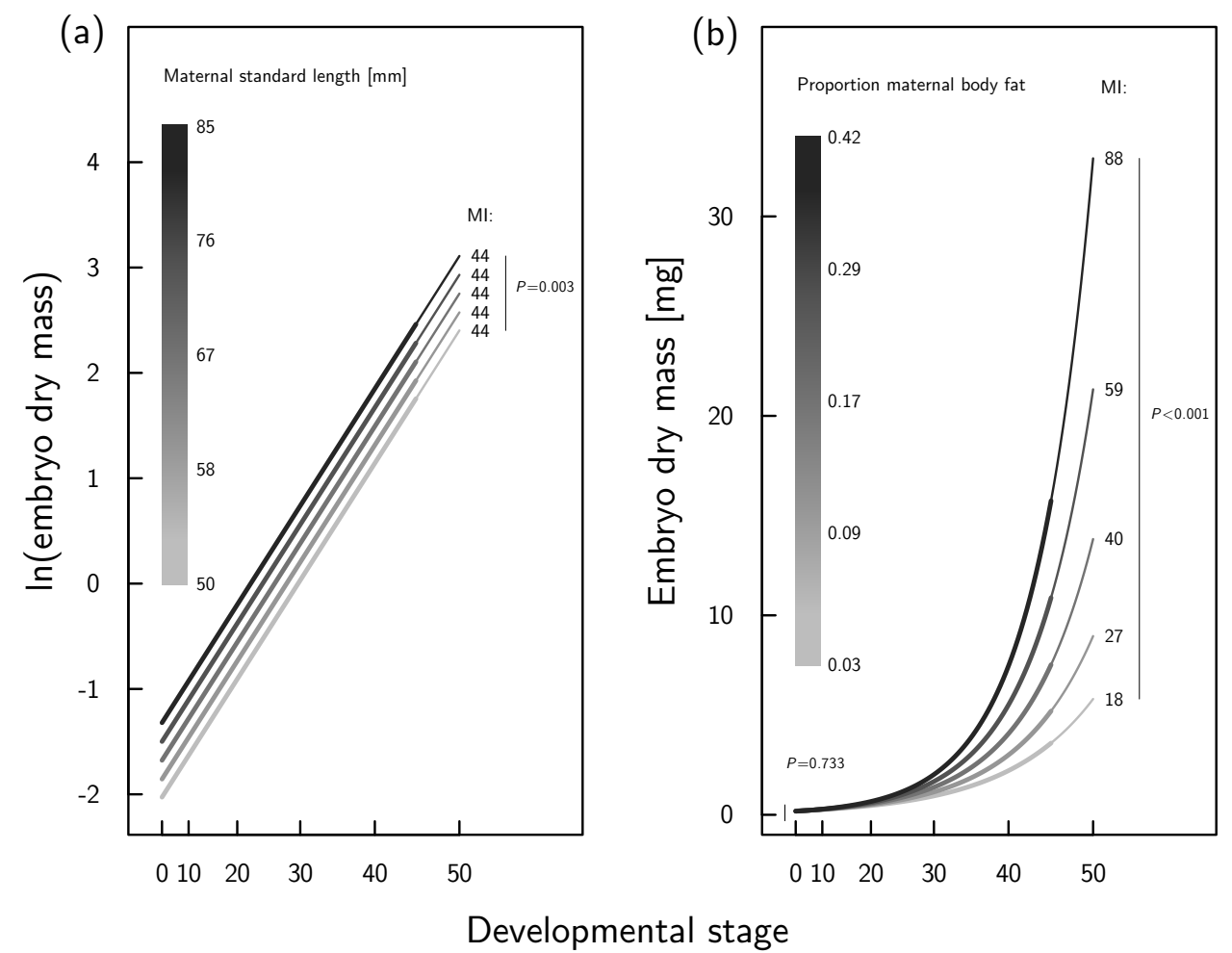

Fig. 2.3. (a) The relationship between maternal standard length and embryo dry mass throughout gestation. Embryo dry mass is predicted as a function of developmental stage, for the population mean of the proportion of maternal body fat (i.e. $\bar{y}_{\text {body fat }}=0.20$ ), and for five hypothetical females of different length (i.e. $50,58,67,76$, and $85 \mathrm{~mm}$ ). The prediction is based on the model that best describes variation in embryo dry mass according to the AICc ( $n=68$ broods from 40 mothers; Table S.2.2, Online Table S3). To illustrate the proportional association of maternal standard length with egg dry mass at fertilization and offspring dry mass at birth, both axes are shown on the same scale as used to fit the model (i.e. $y$-axis: In-scale, $x$-axis: $\sqrt{x^{3}}$ ). Please note that the $y$-intercepts on In-scale are significantly different $\left(t_{30.025}=3.214, P=0.003\right)$, while the slopes are the same. This suggests that female length is proportionally associated with egg mass at fertilization and offspring mass at birth, which means that the predicted matrotrophy indices (shown on the right) remain unaffected (i.e. $\mathrm{MI}=44$ for all five females). The thin-lined parts correspond to the time of birth (i.e. developmental stage 45-50). (b) The interaction effect between the proportion of maternal body fat and developmental stage of embryos estimated in the best model explaining variation in embryo dry mass according to the AICc ( $n=68$ broods from 40 mothers; Table S.2.2, Online Table S3). Embryo dry mass is predicted as a function of developmental stage, for the population mean of maternal standard length (i.e. $\bar{y}_{\text {standard length }}=67$ $\mathrm{mm}$ ), and for five hypothetical females with different proportions of body fat (i.e. $0.03,0.09,0.17$, 0.29 , and 0.42 proportion body fat). The $x$-axis is shown on the same scale as used to fit the model (i.e. $\sqrt{x^{3}}$ ). The $y$-axis is shown on the un-transformed scale to illustrate the exponential increase in embryo dry mass. Please note that the y-intercepts are not significantly different $\left(t_{54.291}=\right.$ $-0.343, P=0.733)$, while the slopes differ significantly among the five females $\left(t_{64.077}=3.723\right.$, $P<0.001$; Online Table S3). This indicates that maternal body fat is not related to the amount of pre-fertilization maternal provisioning, but strongly correlates with the amount of post-fertilization provisioning to the embryo during pregnancy and hence offspring dry mass at birth and the $\mathrm{MI}$ $\left(t_{74.654}=5.369, P<0.001\right)$. 

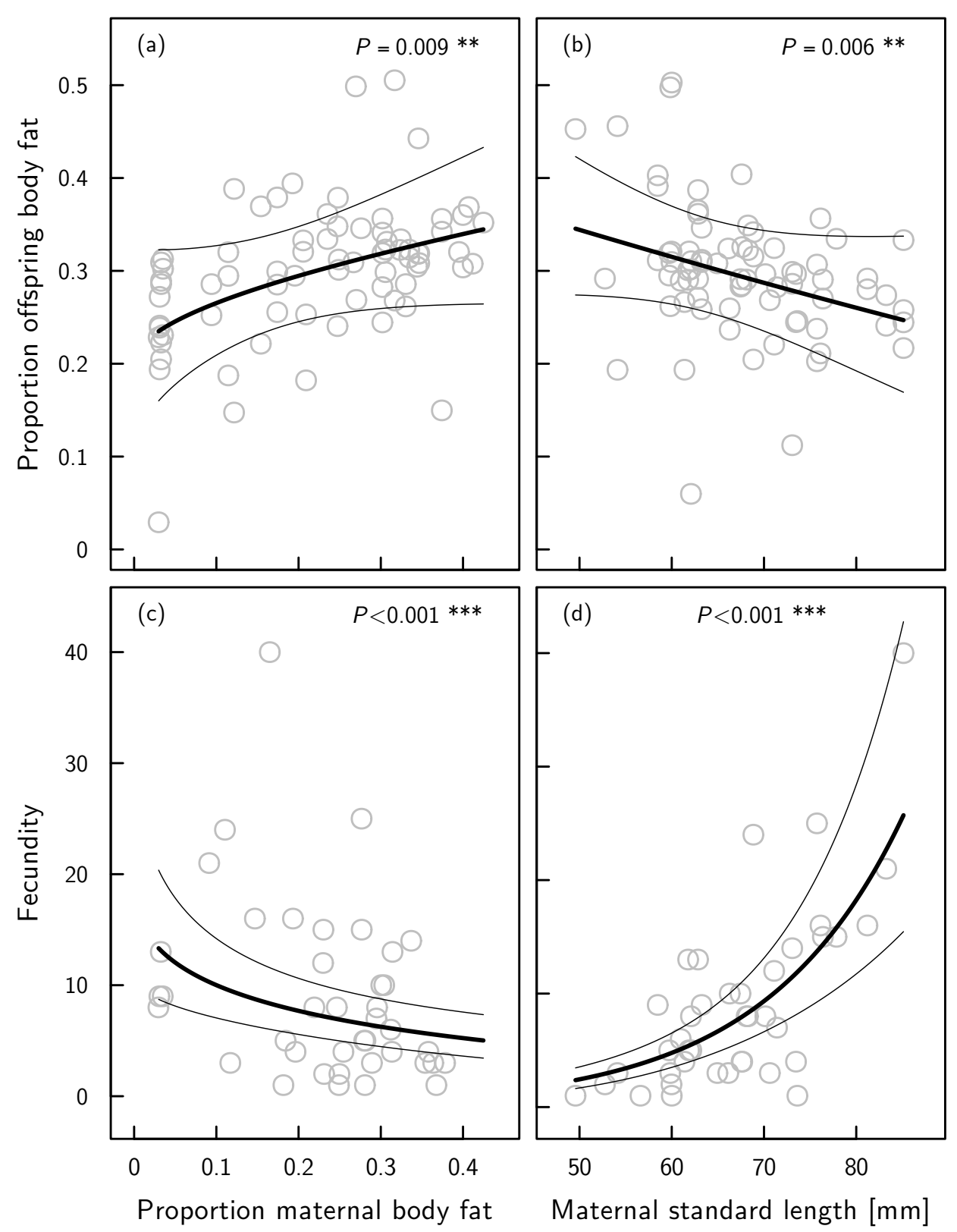

Fig. 2.4. The top panel shows the association of (a) the proportion of maternal body fat and (b) maternal standard length with the proportion of offspring body fat at birth $( \pm 95 \% \mathrm{Cl})$ estimated in the model described in Online Table S6.b. Residuals and model fit account for maternal standard length in (a) and the proportion of maternal body fat in (b) that are kept constant at their population mean (i.e. $\bar{y}_{\text {standard length }}=67 \mathrm{~mm}, \bar{y}_{\text {body fat }}=0.20$ ). The bottom panel shows the association of the number of embryos (i.e. fecundity) with (c) proportion of maternal body fat, and $(d)$ maternal standard length $( \pm 95 \% \mathrm{Cl})$ estimated in the model described in Online Table S7. Model fit accounts for maternal standard length in (c) and the proportion of maternal body fat in (d) that are kept constant at their population mean (i.e. $\bar{y}_{\text {standard length }}=67 \mathrm{~mm}$, $\left.\bar{y}_{\text {body fat }}=0.20\right)$. 


\subsubsection{Maternal effects on proportion of embryo body fat}

The proportion of embryo body fat also increases significantly throughout gestation $\left(t_{74}=\right.$ 2.084, $P=0.041$; Online Table S6). Maternal standard length is negatively related to the proportion of embryo body fat throughout development $\left(t_{74}=-2.849, P=0.006\right.$; Online Table S6; Fig. 2.4b). The proportion of maternal body fat is positively associated with the proportion of offspring body fat at birth $\left(t_{74}=2.675, P=0.009\right.$; Online Table S6.b; Fig. 2.4a), but does not correlate with the proportion of egg fat at fertilization $\left(t_{74}=-0.109, P=0.914\right.$; Online Table S6.a $)$.

\subsubsection{Fecundity}

Both the proportion of maternal body fat and standard length were significantly associated with maternal fecundity (Online Table S7). Maternal fecundity is negatively related to an increasing proportion of maternal body fat $(z=-4.022, P<0.001$; Online Table S7; Fig. 2.4c) and strongly positively related to an increasing standard length $(z=7.606$, $P<0.001$; Online Table S7; Fig. 2.4d).

\subsection{Discussion}

Maternal traits can profoundly influence the development and hence phenotype of offspring at birth (Mousseau \& Fox, 1998a,b). We studied the associations of maternal traits with offspring provisioning prior to and during gestation in field-collected specimens of the matrotrophic live-bearing fish species Poeciliopsis retropinna (family Poeciliidae). We found temporal variation in the composition of embryos throughout gestation: while lean mass is continuously accumulating, fat reserves are predominantly allocated to embryos very late in pregnancy (Fig. 2.2). Furthermore, we found that the level of maternal provisioning to the developing embryos correlates with the proportion of maternal body fat and standard length, but not with maternal lean mass. The proportion of maternal body fat is positively associated with offspring dry mass at birth, but not with egg dry mass at fertilization. This suggests that an increase in the proportion of maternal body fat is likely associated with an increase in the matrotrophy index (MI). By contrast, maternal standard length is proportionally associated with both egg dry mass at fertilization and offspring dry mass at birth, indicating that the MI does not change with increasing maternal standard length (Fig. 2.3a,b).

\subsubsection{Effect of maternal body fat}

We found that the proportion of maternal body fat in $P$. retropinna is associated with an increase in post- but not pre-fertilization maternal provisioning. Females that have more fat reserves produce larger offspring at birth than females with lower fat reserves, without investing more in egg size at fertilization. Consequently, females with more fat reserves 
are likely to have higher 'levels of matrotrophy', or in other words, higher MIs. The potential mechanisms behind the increased level of post-fertilization maternal provisioning by better-conditioned mothers is unclear. In general, the amount of resources a female can transfer to her developing offspring per unit of time is likely the result of a balance between maternal energy uptake (via feeding), her own caloric utilization (maintenance) and the amount of excess energy that is subsequently available for reproduction (Stearns, 1992). It is possible that better-conditioned females that carry more fat reserves simply have more energy available that can be used to invest in developing embryos. In this case, rather than being an adaptive strategy, worse-conditioned females would be physiologically hindered to produce larger offspring.

Better-conditioned mothers (i.e. those carrying larger fat reserves) produced fewer, but better-conditioned offspring. One potential explanation for this is the trade-off between offspring size and number; the production of large and high-quality offspring may necessarily entail the production of fewer offspring owing to the limited size of the female body cavity (Stearns, 1992). Moreover, variation in offspring size and composition at birth is likely to have profound effects on offspring fitness, since larger offspring size at birth and a higher proportion of offspring body fat both have been shown to improve survival under specific environmental conditions. Larger offspring, for instance, perform better when food is scarce and competition for food is high, though the advantage of being relatively larger disappears when competition is insignificant (Parker \& Begon, 1986; Bashey, 2008; Leips et al., 2013). In fish, larger offspring have better escape performance (Gibb et al., 2006; Dial et al., 2016), which is likely to increase survival in high predation environments. Similarly, larger offspring are more resistant to starvation (Gliwicz \& Guisande, 1992), probably because larger offspring contain more maternal reserves that prevent them from starvation under adverse food conditions (Tessier et al., 1983). In situations where the maternal environment provides a reliable predictor of future environmental conditions that offspring are likely to experience, females may evolve the ability to adaptively adjust offspring phenotype at birth as a response to environmental cues (Mousseau \& Fox, 1998b). Matrotrophic fish, for instance, have been shown to increase offspring size in response to increasing conspecific densities (Leips et al., 2013). This pattern is interpreted as adaptive as it increases offspring fitness under highly competitive conditions.

Here we show that the maternal phenotype is strongly correlated with offspring phenotype in a matrotophic fish; offspring size and composition at birth is strongly associated with maternal body condition. Although we cannot determine whether offspring size and composition at birth is due to adaptive transgenerational plasticity or morphological and/or physiological constraints, the observed pattern of differential embryo provisioning is seemingly more consistent with a physiological constraint. In matrotrophic fish, it has been proposed that maternal fat reserves buffer females against low or fluctuating food availability (Trexler, 1997; Trexler \& DeAngelis, 2003). Since matrotrophic species continuously supply their developing embryos with resources (Wourms, 1981; Pollux et al., 2009), the timing of determining brood size and offspring size are likely to be decoupled 
(Reznick et al., 1996b; Pollux \& Reznick, 2011). Whereas brood size is determined prior to fertilization based on current food availability, offspring size is determined after fertilization based on food availability throughout gestation (Reznick et al., 1996b; Pollux \& Reznick, 2011). In environments where food is scarce or fluctuating, therefore, matrotrophic species presumably run the risk of fertilizing more eggs than can be thoroughly provisioned during gestation. Stored maternal fat reserves could theoretically prevent females from undernourishing their embryos and enable them to sustain their brood under adverse food conditions (Trexler, 1997; Trexler \& DeAngelis, 2003). However, available evidence suggests that maternal body fat might not fully buffer matrotrophic females against unfavorable environmental conditions (Pollux \& Reznick, 2011). The matrotrophic fish species Heterandria formosa, Poeciliopsis turneri, P. prolifica and Phalloptychus januarius all responded to low food conditions under laboratory conditions by producing smaller offspring at birth with less fat reserves (Reznick et al., 1996b; Pollux \& Reznick, 2011). In concurrence with these laboratory studies, we show, under natural conditions in fieldcollected specimens of Poeciliopsis retropinna, that decreasing maternal body condition (i.e. proportion body fat) is negatively associated with offspring size and body condition at birth. Since smaller offspring with less fat reserves are expected to have lower fitness under adverse food and hence highly competitive conditions (Tessier et al., 1983; Parker \& Begon, 1986; Bashey, 2008; Leips et al., 2013), matrotrophy has been suggested to be a maladaptive strategy in environments characterized by low or fluctuating food availability (Reznick et al., 1996b; Pollux \& Reznick, 2011).

\subsubsection{Timing of fat allocation to developing embryos}

Live-bearing fish larvae from the family Poeciliidae are super precocial, having functional prey capture abilities at birth (Lankheet et al., 2016). The newborn's prey capturing ability, however, is far from perfect and requires a rapid integrated development of the visuo-motor system during the first days after birth to optimize prey capture success rate and ensure a sufficient uptake of resources for survival (Lankheet et al., 2016). This is particularly important when offspring are born in low resource environments where prey availability may be sparse. In these environments, offspring may benefit from having a 'back pack with fat reserves' that may help them to survive the first days after birth (Chambers et al., 1989).

We found that embryos from the matrotrophic fish species Poeciliopsis retropinna gain fat only very late in pregnancy. The same pattern has been shown in placental mammals, where fetal fat mobilization also occurs late in pregnancy (Petterson et al., 1994). In mammals, fetal fat deposition during pregnancy is however relatively insignificant (Elphick et al., 1979), since most lipids are allocated to offspring postnatally, i.e. during lactation (Bell, 1995). One might argue that the late (in fish) or relatively insignificant (in mammals) allocation of fat during pregnancy could be an adaptive feature of mobile matrotrophic live-bearing animals in general, because studies have shown that an increase 
in reproductive allocation (i.e. the proportion of the mother's mass allocated to developing offspring) during pregnancy can lead to a less slender body shape (Fleuren et al., 2018) and negatively affect female locomotor performance in a wide range of viviparous taxa (e.g. fish, Plaut 2002; Reznick et al. 2004; reptiles, Seigel et al. 1987; and mammals, Noren et al. 2011), which consequently may reduce survival probability (Plath et al., 2011; Laidlaw et al., 2014). Thus, we suggest that the late allocation of fat might be adaptive, because earlier allocation of resources would unnecessarily increase the reproductive burden suffered by a pregnant female, and negatively impact her locomotor performance and, hence, her chance of survival.

\subsubsection{Effect of maternal size}

Larger (i.e. longer) females had greater fecundity. This finding is consistent with patterns observed in a wide range of taxa that exhibit indeterminate growth (Reznick, 1983), since fecundity increases as a consequence of space available in the female's body cavity (Shine, 1992). In addition, we found that maternal standard length is positively correlated with offspring size at birth; although this effect is approximately three times smaller than the effect of the proportion of maternal body fat $\left(\beta_{\mathrm{SL}}^{\prime}=0.098\right.$ versus $\left.\beta_{\mathrm{fat}}^{\prime}=0.279\right)$. Larger females produced more and larger offspring at birth. Optimality models predict that the total reproductive effort will increase with age, as expected future reproductive success decreases (Pianka \& Parker, 1975). Since standard length is positively correlated with age (Reznick et al., 1996a), the production of more and larger offspring by larger females might be explained by an increased reproductive investment with age, rather than by female size per se.

We further found that maternal length is negatively related with offspring body condition. Larger females produce more and larger offspring, but they contain less fat reserves. As a female's physiology changes during ageing, offspring quality might be expected to decrease with maternal age as a consequence of senescence. Offspring of old females have been shown to have reduced survival probability (Descamps et al., 2008), lower egg hatching success (Kern et al., 2001), and lower fat reserves (McIntyre \& Gooding, 2000). Although the total reproductive investment (i.e. size and number of offspring) is likely to increase with maternal age as a consequence of decreasing future reproductive success (Pianka \& Parker, 1975), older mothers might provide their offspring with less fat reserves as a consequence of senescence. Since we do not have direct measurements of a female's age, however, we cannot disentangle maternal size effects due to morphological constraints (i.e. body cavity volume) and maternal age effects due to senescence and/or age-dependent reproductive effort.

\subsubsection{The matrotrophy index is (at least partly) a phenotypically plastic trait}

Our results strongly suggest that the MI exhibits phenotypic plasticity and can change throughout a female's lifetime (i.e. due to maternal body condition but not length) 
or across environmental conditions (e.g. due to food availability). The extent of postfertilization maternal provisioning has already been shown to plastically respond to environmental conditions such as food availability in other matrotrophic poeciliids (Reznick et al., 1996b; Pollux \& Reznick, 2011). Specifically, females produced smaller offspring at birth under low food availability. This is likely to reduce the MI. However, in these studies measurements of the egg phenotype (i.e. the extent of pre-fertilization maternal provisioning) were either not reported or analyzed, and consequently it was not possible to make inferences about the MI. Nevertheless, these studies show an almost instantaneous link between food availability and offspring size at birth. Here we demonstrate that offspring size at birth strongly correlates with maternal body condition and that maternal body condition relates to the MI primarily through post-, rather than pre-fertilization maternal provisioning. The extent to which maternal provisioning is a direct consequence of food availability (i.e. nutrients that are provided without transit through maternal fat reserves) and an indirect consequence mediated through maternal body condition (i.e. nutrients that are mobilized from maternal fat reserves) remains to be tested. Whereas under nutrient-rich conditions females may provision their embryos with resources that are not previously stored as maternal fat, they may increasingly rely on fat reserves when food is scarce, making body condition an important and direct embryonal food source under adverse conditions.

\section{S.2 Supplementary Information}

Additional supplementary information can be found in the online version of the article: https://onlinelibrary.wiley.com/doi/full/10.1002/ece3.4542

\section{S.2.1 Supplementary Methods}

Table S.2.1. Sampling locations (plus coordinates) in the Rio Terraba (Costa Rica), sampling dates and number of collected $(n)$ and pregnant $\left(n_{\text {preg }}\right)$ females at each location.

\begin{tabular}{|c|c|c|c|c|}
\hline Location & Coordinates & Sampling date & $n$ & $n_{\text {preg }}$ \\
\hline Pacuar & $\mathrm{N} 09^{\circ} 21^{\prime}, \mathrm{W} 83^{\circ} 44^{\prime}$ & 25.03 .2013 & 20 & 3 \\
\hline Pedregoso & $\mathrm{N} 09^{\circ} 21^{\prime}, \mathrm{W} 83^{\circ} 43^{\prime}$ & 25.03 .2013 & 30 & 0 \\
\hline Ceibo & $\mathrm{N} 09^{\circ} 09^{\prime}, \mathrm{W} 83^{\circ} 22^{\prime}$ & 26.03.2013 & 24 & 8 \\
\hline Sucio & $\mathrm{N} 08^{\circ} 49^{\prime}, \mathrm{W} 82^{\circ} 55^{\prime}$ & 27.03.2013 & 24 & 23 \\
\hline Copal & $N 08^{\circ} 48^{\prime}, W 82^{\circ} 55^{\prime}$ & 27.03 .2013 & 10 & 6 \\
\hline
\end{tabular}




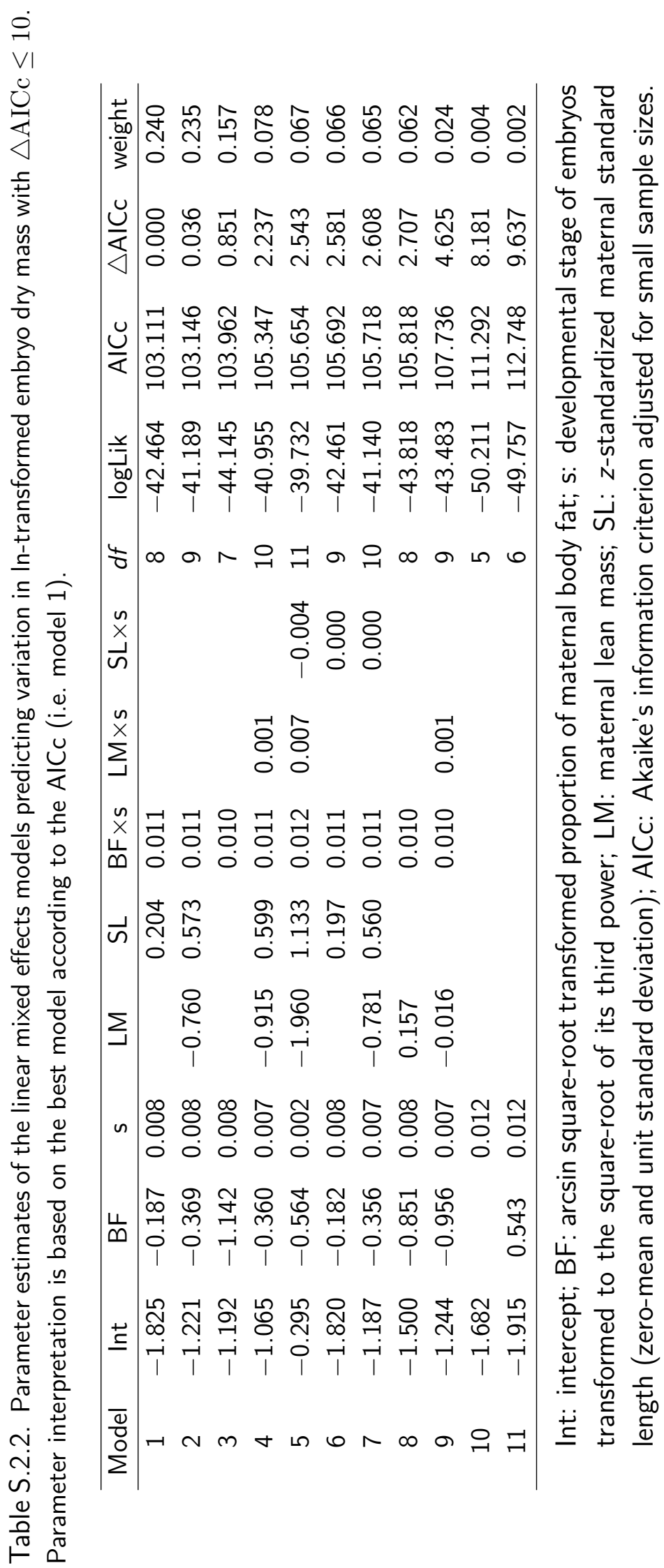




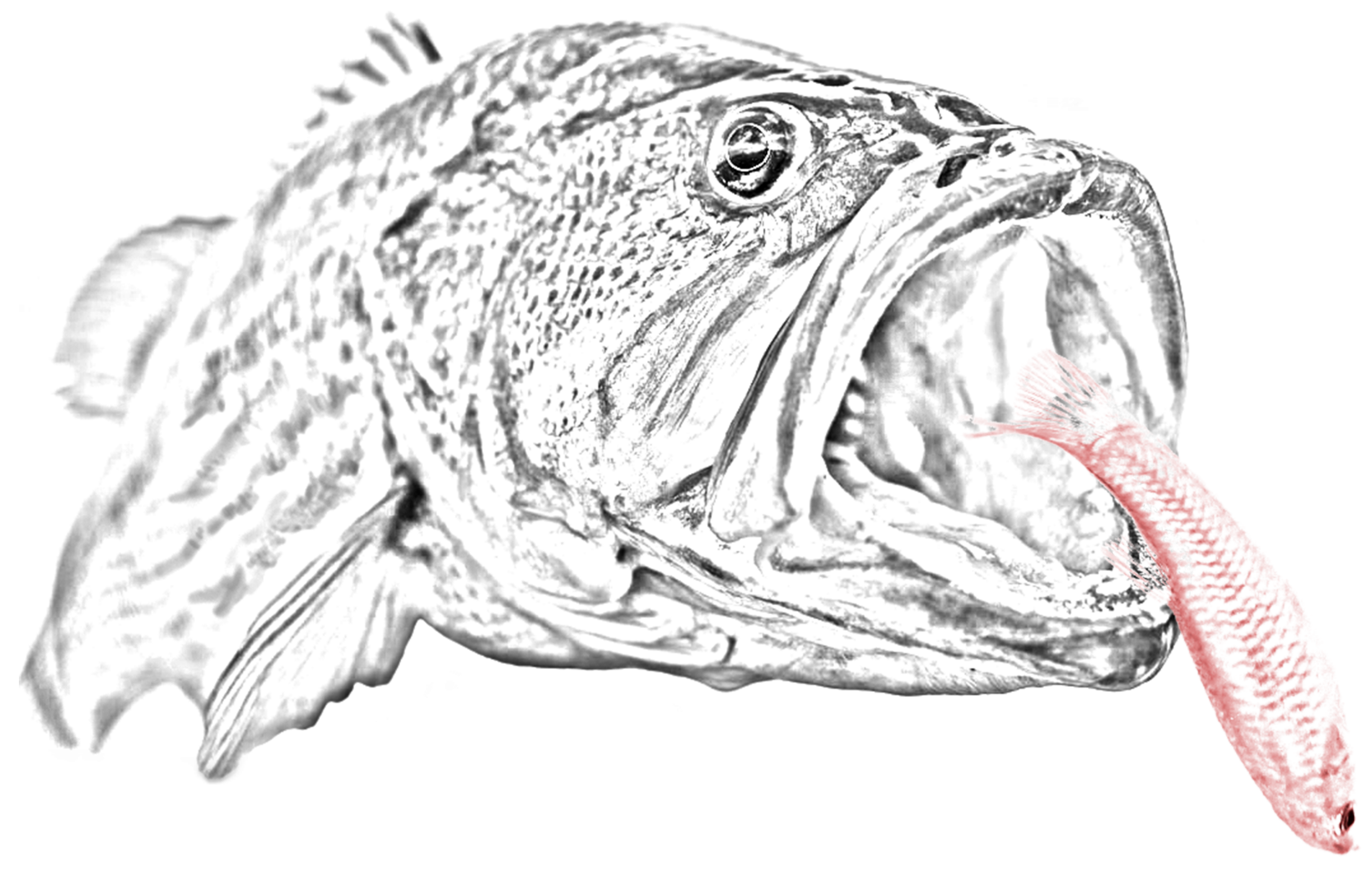




\section{Predation risk shapes the degree of placen- tation in natural populations of live-bearing fish}

Andres Hagmayer ${ }^{1}$, Andrew I. Furness ${ }^{2,3}$, David N. Reznick ${ }^{4}$, Myrthe L. Dekker ${ }^{1} \&$ Bart J.A. Pollux ${ }^{1}$

1 Department of Animal Sciences, Wageningen University, 6708 WD Wageningen, The Netherlands

2 Department of Ecology and Evolutionary Biology, University of California, CA 92697 Irvine, USA

3 Department of Biological and Marine Sciences, University of Hull, HU6 7RX Hull, UK

${ }^{4}$ Department of Biology, University of California, CA 92521 Riverside, USA

This chapter has been published as: Hagmayer, A., et al. (2020). Predation risk shapes the degree of placentation in natural populations of live-bearing fish. Ecology Letters, 23(5):831-840. https://doi .org/10.1111/ele.13487 


\section{Abstract}

The placenta is a complex life-history trait that is ubiquitous across the tree of life. Theory proposes that the placenta evolves in response to high performance-demanding conditions by shifting maternal investment from pre- to post-fertilization, thereby reducing a female's reproductive burden during pregnancy. We test this hypothesis by studying populations of the fish species Poeciliopsis retropinna in Costa Rica. We found substantial variation in the degree of placentation among natural populations associated with predation risk: females from high predation populations had significantly higher degrees of placentation compared to low predation females, while number, size and quality of offspring at birth remained unaffected. Moreover, a higher degree of placentation correlated with a lower reproductive burden and hence likely an improved swimming performance during pregnancy. Our study advances an adaptive explanation for why the placenta evolves by arguing that an increased degree of placentation offers a selective advantage in high predation environments.

Keywords: life-history, live-bearing, matrotrophy, placenta, placentotrophy, Poeciliidae, predation, superfetation, Trexler-DeAngelis, viviparity

\subsection{Introduction}

Understanding the origin and elaboration of complex traits is of fundamental interest to evolutionary biologists. Placentas are complex organs that have independently evolved many times throughout the animal kingdom in widely divergent lineages (Wourms, 1981; Wooding \& Burton, 2008; Blackburn, 2015; Wake, 2015; Ostrovsky et al., 2016). This repeated evolution points to a possible adaptive advantage (Losos et al., 1998), however, the nature of this advantage remains elusive.

To date, two adaptive hypotheses (termed the resource allocation and locomotor cost hypotheses) provide potential explanations for why the placenta evolves (i.e. its selective advantage). Both hypotheses treat the placenta as a life-history adaptation that evolves in response to ecological selection pressures (Thibault \& Schultz, 1978; Trexler \& DeAngelis, 2003). Furthermore, both hypotheses posit that the ancestral state is for all nutrients to be prepackaged in the form of egg yolk (lecithotrophy) and that the derived state is for nutrients to be supplied to embryos throughout pregnancy via a placenta (placentotrophy). This is presumably achieved via a gradual shift in the timing of nutrient provisioning from pre- to post-fertilization. If true, then the evolution of the placenta should coincide with a reduction in egg mass at fertilization without affecting the total investment per neonate at birth (Reznick et al., 2002; Trexler \& DeAngelis, 2003; Pollux et al., 2009). The live-bearing fish family Poeciliidae is a useful system in which to test these ideas, because it contains species that span a continuum of pre- versus post-fertilization nutrient provisioning, ranging from strictly lecithotrophic to highly placentotrophic. In addition, 
placentotrophy has evolved independently numerous times in this family (Reznick et al., 2002; Pollux et al., 2014; Furness et al., 2019).

The resource allocation hypothesis (Trexler \& DeAngelis, 2003) posits that the evolution of the placenta permits females to produce greater brood sizes and hence attain higher fecundity than lecithotrophic females when sufficient resources are available to carry the developing embryos to term. Moreover, placental females rely on a steady nutritional supply to provision embryos throughout pregnancy. Trexler \& DeAngelis (2003) therefore argued that the ability to selectively abort embryos and recycle this investment, should resource conditions suddenly deteriorate, is a crucial preadaptation for the evolution of placentation. Studies in the Poeciliidae have not shown that they are able to do this, suggesting that the conditions under which the placenta might be favoured by natural selection are restricted to environments characterized by high and stable resource conditions (Reznick et al., 1996b; Trexler, 1997; Banet \& Reznick, 2008; Banet et al., 2010; Pollux \& Reznick, 2011).

The locomotor cost hypothesis (Thibault \& Schultz, 1978; Pollux et al., 2009; Pires et al., 2011) postulates that the placenta evolves to offset some of the locomotor costs associated with a live-bearing mode of reproduction. The physical and physiological burden of pregnancy negatively affects a female's locomotor performance in a broad range of live-bearing animals (Seigel et al., 1987; Plaut, 2002; Noren et al., 2011; Fleuren et al., 2019). The evolution of the placenta should allow females to attain higher fitness, because the production of smaller eggs at fertilization reduces a female's reproductive burden during pregnancy. This improves body streamlining and locomotor performance, notably without sacrificing reproductive output. The presumed benefit of a higher degree of placentation is that the improved swimming performance offers a selective advantage to females in performancedemanding (e.g. high predation) environments because it enhances survival probability (Pollux et al., 2009; Pires et al., 2011). Whereas the resource allocation hypothesis has already been the focus of several empirical studies (Banet \& Reznick, 2008; Banet et al., 2010; Pires et al., 2011; Pollux \& Reznick, 2011; Bassar et al., 2014), the locomotor cost hypothesis has not yet been subject to similar systematic investigation.

Here, we quantify the degree of placentation in high and low predation populations of the placental live-bearing fish species Poeciliopsis retropinna (family Poeciliidae, Regan 1908) in Costa Rica. We test a key prediction of the locomotor cost hypothesis, which is that placentation evolves in performance-demanding (high predation) environments. If the placenta is favored under high predation conditions, we should find a higher degree of placentation in high predation populations. Theory further predicts that this higher degree of placentation should be due to a smaller egg size at fertilization without affecting the number, size, or quality of offspring at birth, translating to a lower reproductive burden for females during pregnancy (Thibault \& Schultz, 1978; Pollux et al., 2009; Pires et al., 2011). Finally, we discuss the potential fitness advantage of evolving a higher degree of placentation in high predation populations. 


\subsection{Material and methods}

\subsubsection{Study species and collection sites}

Poeciliopsis retropinna is found in freshwater streams of varying water velocity and predation pressure in Costa Rica and Panama (Bussing, 2002). They are observed in habitats with low predation risk (no strongly piscivorous species present), or co-occurring with one or more piscivorous predator species: Parachromis dovii, Eleotris picta, and Gobiomorus maculatus. During gestation, P. retropinna females transfer nutrients to developing embryos via a placenta. The degree of post-fertilization maternal provisioning in this species is extensive, with offspring increasing in dry mass more than 100-fold during gestation $(\mathrm{MI}=117)$ (Reznick et al., 2002). Moreover, P. retropinna has superfetation, the ability to carry several broods at different developmental stages.

During February and March 2013, 2017, and 2018, P. retropinna were collected at 27 different locations in the Rio Terraba and Rio Coto drainages in the province of Puntarenas, Costa Rica (Table S.3.1). Two of the locations were repeatedly sampled resulting in 29 study populations. When water visibility was high (i.e. in all but one location), the occurrence of piscivorous predator species was most effectively detected using underwater visual census by three independent snorkeler. Censuses took place during daytime $(\sim 3-5$ hours per location/snorkeler) at different positions along each river. In one location (Rio Conte), water visibility was low and predator community was assessed using seine and cast nets. We found 17 'high' predation localities where piscivorous predator species were present, and 12 'low' predation localities where predators were absent (Table S.3.1). At each location, 5-37 adult females were collected, euthanized with an overdose of MS-222, and preserved in $5 \%$ formaldehyde.

\subsubsection{Laboratory measurements}

Maternal standard length and the proportion of body fat were measured using established protocols (Supplementary Methods S.3.1.1). 28 of the 29 sampled populations contained pregnant females (Table S.3.1), therefore all subsequent anatomical and statistical analyses were carried out only with females from 28 populations $\left(n_{\text {preg }}=463\right)$. The ovaries were dissected to count the total number of embryos (fecundity), regressors (aborted embryos), broods at different developmental stages (superfetation), embryos in a brood (brood size), and to determine the developmental stage and average dry mass of embryos in a brood (Table 3.1). The developmental stages are based on morphological criteria described in Haynes (1995) and range from 0 (eggs at fertilization, no development) to 45 (fully-developed embryos). Fecundity was calculated by excluding stage 0 embryos, because it was difficult to assess if 0-staged eggs were fertilized or not. Instead, embryos at developmental stage 2 , rather than 0 , were defined as 'eggs at fertilization'. 
Table 3.1. Summary of maternal life-history traits.

\begin{tabular}{|c|c|}
\hline \multicolumn{2}{|r|}{ Maternal life-history traits } \\
\hline Egg mass at fertilization & Dry mass of eggs at fertilization (i.e. developmental stage 2) \\
\hline Offspring mass at birth & Dry mass of fully-developed embryos (i.e. developmental stage 45) \\
\hline Proportion egg fat & Egg fat at fertilization divided by dry mass of eggs at fertilization \\
\hline Proportion offspring fat & Offspring fat at birth divided by offspring dry mass at birth \\
\hline Reproductive allotment & $\begin{array}{l}\text { Proportion of the mother's dry mass allocated to reproduction (i.e. } \\
\text { embryo dry mass, regressor dry mass, and placental dry mass) }\end{array}$ \\
\hline $\begin{array}{l}\text { Absolute reproductive } \\
\text { allotment }\end{array}$ & $\begin{array}{l}\text { Total dry mass allocated to reproduction (i.e. embryo dry mass, regres- } \\
\text { sor dry mass, and placental dry mass) }\end{array}$ \\
\hline Brood size & Number of embryos in a given brood \\
\hline Fecundity & $\begin{array}{l}\text { Number of embryos carried by a female counted across all broods ex- } \\
\text { cluding stage } 0 \text { embryos }\end{array}$ \\
\hline Superfetation & Number of broods at different developmental stages \\
\hline Abortion incidence & $\begin{array}{l}\text { Number of regressors (i.e. aborted embryos) divided by the sum of the } \\
\text { number of regressors and embryos }\end{array}$ \\
\hline
\end{tabular}

\subsubsection{Quantification of the degree of placentation}

The Matrotrophy Index (MI), calculated as the ratio of offspring mass at birth to egg mass at fertilization, was used as an unbiased measure of the degree of placentation (Reznick et al., 2002). Some live-bearing species allocate all resources to eggs prior to fertilization in the form of large fully-yolked eggs (termed lecithotrophy). These embryos lose dry mass over the course of gestation due to metabolic processes, leading to an $\mathrm{MI}<1$. Other species allocate nutrients to the developing offspring post-fertilization throughout pregnancy (termed matrotrophy). Such species have an MI > 1, indicating embryos gain dry mass during pregnancy. Placentotrophy represents one specific type of matrotrophy that is achieved through a follicular placenta, roughly an analog to the mammalian placenta (Pollux et al., 2009). Because the MI is determined by both egg mass at fertilization and offspring mass at birth, an increase in the degree of placentation can be brought about by an increase in offspring mass at birth and/or a decrease in egg mass at fertilization.

The MI for P. retropinna was estimated in relation to (i) a given population in a specific year, and (ii) high and low predation risk by using the Bayesian programming environment JAGS (Plummer, 2003) in R v 3.5 (R Core Team, 2020) (Supplementary Methods S.3.1.2, S.3.1.3).

In (i), ln-transformed embryo dry mass was fitted as a function of the developmental stage of embryos (stage), stage $^{2}$, proportion of maternal body fat (BF), maternal standard length (SL), and BF $\times$ stage. For each population, the model estimates year-specific intercepts and slopes on stage. This allows for the prediction of MI for a given population in a specific year. In addition, the model includes mother identity as additional intercept 
to allow for variation among females that is not accounted by maternal BF and SL. The population-specific MI's were subsequently calculated by dividing offspring mass at birth (stage 45) by egg mass at fertilization (stage 2) that were predicted for a given population in a specific year and for a female of overall average SL and BF. Since the MI's are predicted for a female of the same SL and BF, the resulting MI's are independent of these traits (Fig. S.3.1).

In (ii), ln-transformed embryo dry mass was fitted as a function of stage, stage ${ }^{2}$, predation, $\mathrm{BF}, \mathrm{SL}, \mathrm{BF} \times$ stage, and predation $\times$ stage. For each population, the model estimates yearspecific intercepts to account for systematic differences among populations within a year. Moreover, the model includes mother identity as additional intercept to allow for variation among females that is not accounted for by maternal BF and SL. The MI for high and low predation females was subsequently calculated by dividing offspring mass at birth by egg mass at fertilization that were predicted for a given predation regime and for a female of overall average SL and BF.

\subsubsection{Quantification of life-history variation among predation regimes}

The effects of predation on life-history traits (egg mass at fertilization, offspring mass at birth, proportion of egg and offspring fat, reproductive allotment, brood size, fecundity, superfetation, and abortion incidence) were analyzed by fitting each trait as a function of (i) high and low predation risk, and (ii) predator community in a series of (generalized) linear mixed effects models in R v 3.5 (R Core Team, 2020), using the package lme4 (Bates et al., 2015). Considering Parachromis dovii (P), Eleotris picta (E), and Gobiomorus maculatus $(\mathrm{G})$ as predator species, we observed six different predation categories in nature: low (no strongly piscivorous species present), G, E, EG, PG, and P. Except in the case of reproductive allotment, additional fixed effects included the proportion of maternal body fat (BF) and standard length (SL). Reproductive allotment is defined as the proportion of the mother's dry mass allocated to reproduction, and hence accounts for female dry mass, rather than BF and SL. Maternal BF predictably responds to experimental manipulation of food availability in the laboratory (Reznick et al., 1996b; Banet \& Reznick, 2008; Banet et al., 2010; Pollux \& Reznick, 2011), and is believed to be a good indicator of fish condition (Leips et al., 2013). Therefore, accounting for maternal BF may enable us to partly decouple the effects of predation risk and food availability on life-history traits. The association of life-history traits with maternal BF and SL is reported and discussed in the Supplementary Information (Online Table S2-S10; Fig. S.3.2). In the case of reproductive allotment, fecundity and superfetation, the developmental stage of the most-developed brood was fitted as an additional fixed effect to account for females early in the reproductive cycle. Population, year, river, and population $\times$ year were fitted as random intercepts accounting for spatio-temporal non-independence of observations. Likewise, in the case of brood size, mother identity was fitted as additional random intercept to correct for pseudo-replication, because brood size is measured multiple times 
in females with superfetation.

To optimize normality and homoscedasticity of model residuals, reproductive allotment, abortion incidence, proportion of egg and offspring fat, and maternal BF were arcsin square-root transformed. Fecundity, superfetation, and brood size were fitted in generalized linear mixed effects models using a log link for the Poisson-distributed responses.

\subsubsection{Path analysis}

Differences in reproductive allotment (RA) among populations could be due to effects on several life-history traits. For instance, RA could be decreased by reducing brood size or superfetation, which in turn decreases the number of embryos (fecundity). Alternatively, RA diminishes when producing smaller eggs at fertilization or offspring at birth. We used a path analysis to determine the contribution of each of these life-history traits to differences in RA among predation regimes. In total, three (generalized) linear mixed effects models, implemented in MCMCglmm (Hadfield, 2010), were used to estimate all paths (Supplementary Methods S.3.1.4). Each model was re-fitted as a function of an intercept only (null model) to compare the deviance information criterion (DIC) of the full model against that of the null model $(\triangle \mathrm{DIC})$.

In the first model, egg mass at fertilization, offspring mass at birth, average brood size for a given mother, and superfetation were fitted in a multivariate model as a function of high and low predation risk allowing for the covariance between the residuals of all responses $(\triangle \mathrm{DIC}=-272.75)$. In the second model, maternal fecundity was fitted as a function of superfetation and average brood size for a given mother, as changes in both brood size and superfetation will affect fecundity $(\triangle \mathrm{DIC}=-388.449)$. The third model subsequently predicts absolute RA as a function of fecundity, egg mass at fertilization, and offspring mass at birth $(\triangle \mathrm{DIC}=-135.094)$.

All three models included the proportion of maternal body fat and standard length as fixed effects. In the case of superfetation and fecundity, the developmental stage of the most-developed brood was an additional fixed effect (see above). To aid convergence, we did not fit a random year effect (which was effectively zero), but otherwise the random effect structure was the same as above. Furthermore, all continuous input variables were $z$-standardized to obtain standardized partial regression coefficients $\left(\beta^{*}\right)$ that take values between -1 and 1 (Schielzeth, 2010). In the case of Poisson-distributed responses (fecundity and superfetation), $\beta^{*}$ was obtained retrospectively by dividing the estimated slope $(\beta)$ by the phenotypic standard deviation of the response variable $\left(\sigma_{Y}\right) . \sigma_{Y}$ was indirectly estimated using the variance of the predicted model fits on the link scale $\left(\sigma_{\log (\hat{Y})}^{2}\right)$ and the pseudo- $R$-squared $\left(R^{2}\right)$ (Menard, 2011):

$$
\sigma_{Y}=\sqrt{\sigma_{\log (\hat{Y})}^{2}+R^{2}}
$$


The effect of predation on RA, mediated through a specific maternal life-history trait, is then given by the direct effect of predation on the life-history trait and its contribution to the RA. This effect is quantified by multiplying $\beta^{*}$ of predation on the life-history trait with $\beta^{*}$ of the life-history trait on RA.

\subsection{Results}

There was a more than two-fold range in the estimated degree of placentation among the 28 natural populations of $P$. retropinna (MI ranging from 14.87 to 32.34; Table S.3.1). Consistent with the locomotor cost hypothesis, we found that $P$. retropinna females from high predation (HP) localities exhibit a significantly higher degree of placentation $\left(\mathrm{MI}_{\mathrm{HP}}\right.$ : mean $=22.86,95 \% \mathrm{CI}=21.30-24.46)$, compared to females from low predation (LP) populations $\left(\mathrm{MI}_{\mathrm{LP}}:\right.$ mean $=19.86,95 \% \mathrm{CI}=18.06-21.73 ; P_{\mathrm{MCMC}}=0.013 ;$ Fig. 3.1; Fig. S.3.3). This lends compelling support to the idea that predation risk may drive the evolution of placentas at a micro-evolutionary level.

Fig. 3.1. The degree of placentation in Poeciliopsis retropinna populations, expressed as the Matrotrophy Index ( $\mathrm{MI} \pm 95 \%$ posterior density $\mathrm{Cl}$ ), in relation to high and low predation risk (piscivorous predator species present or absent, respectively). The $\mathrm{MI}$ is predicted for a female of overall average standard length and proportion of body fat (i.e. body fat $=$ 0.16 , standard length $=53 \mathrm{~mm})$. The posterior Bayesian $P$-value $\left(P_{\mathrm{MCMC}}\right)$ is given at the top.

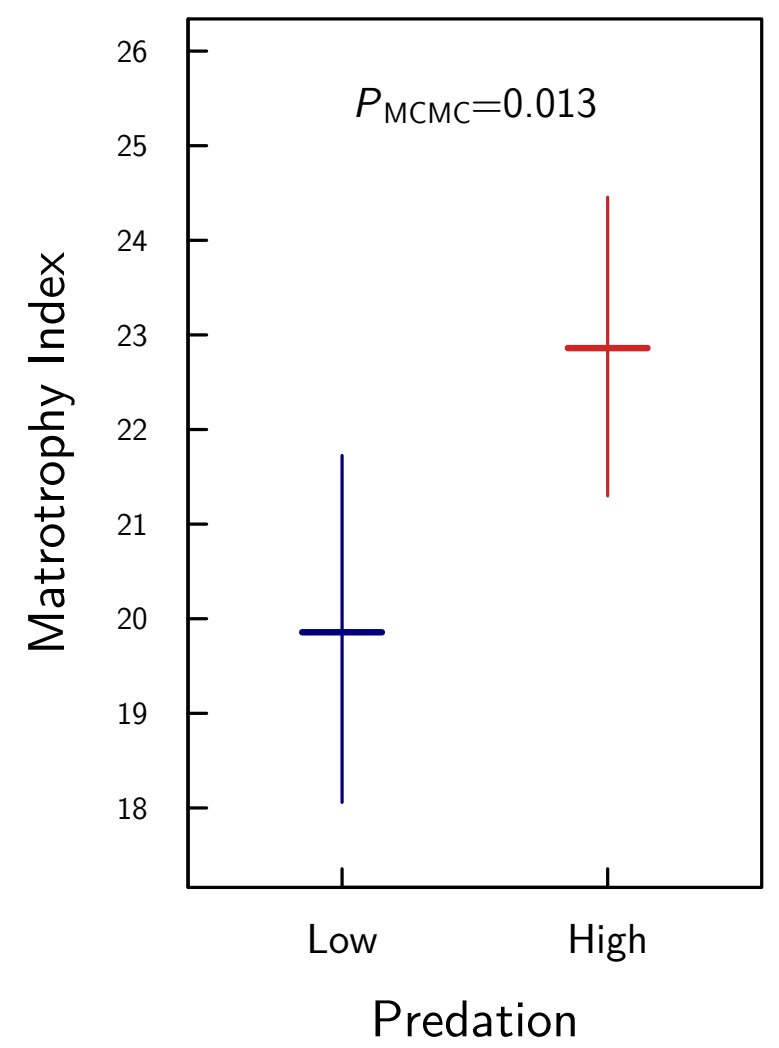



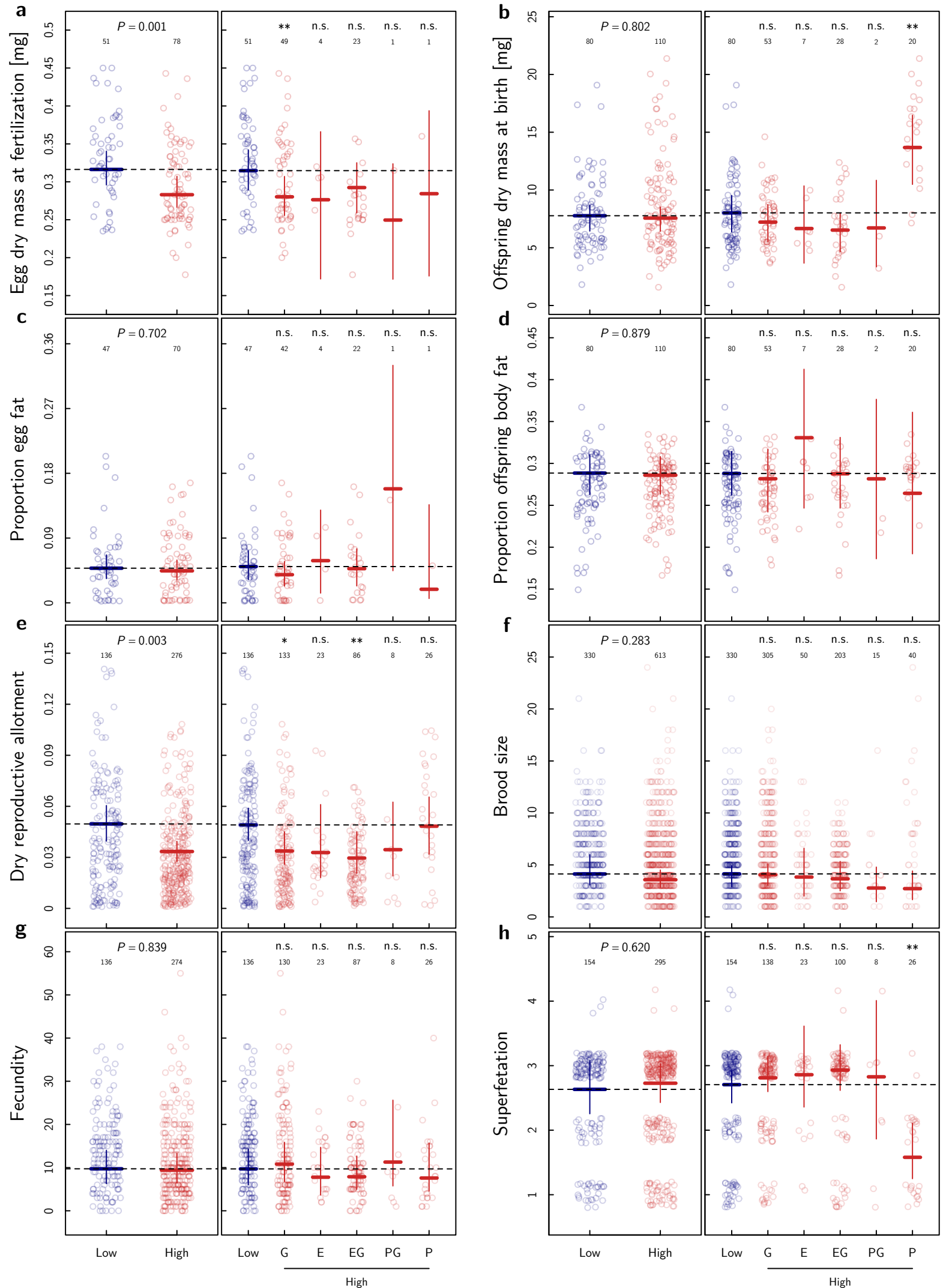

Predation 
Fig. 3.2. Life-history characteristics of Poeciliopsis retropinna in relation to predation risk. a Egg dry mass at fertilization (developmental stage 2), b offspring dry mass at birth (developmental stage 45), c proportion egg fat at fertilization, d proportion offspring fat at birth, e dry reproductive allotment, $\mathbf{f}$ brood size, $\mathbf{g}$ maternal fecundity (number embryos in all broods combined), and $\mathbf{h}$ degree of superfetation $( \pm 95 \% \mathrm{Cl})$ as a function of high and low predation risk (piscivorous predator species present or absent; left panels) and predator community (G: Gobiomorus maculatus; E: Eleotris picta; P: Parachromis dovii; right panels) estimated in the models described in Online Table S2-S9, S22-S29. Except in e, all model predictions account for the proportion of maternal body fat and maternal standard length, which are kept constant at the overall population mean (i.e. body fat $=0.16$, standard length $=53 \mathrm{~mm}$ ). In $\mathbf{e}$ and $\mathbf{g}-\mathbf{h}$, the developmental stage of the mostdeveloped brood carried by the female is kept constant at the overall median (i.e. developmental stage 42.5). Data points (red: high predation; blue: low predation) correspond to the 'jittered' raw data. Sample size and $P$-value are given at the top of each panel. Significant codes: $P<0.001^{* * *}$, $<0.01^{* *}, \leq 0.05^{*},>0.05$ n.s.

However, to test the validity of the locomotor cost hypothesis, at least three additional subpredictions have to be evaluated. First, the higher degree of placentation in HP populations should not be correlated with systematic differences in environmental conditions, because then other local environmental conditions account (at least partly) for the observed inter-population variation in the degree of placentation, either by influencing egg mass at fertilization or offspring mass at birth. We measured several water quality parameters at each location (salinity, water velocity, hardness, $\mathrm{NH}_{4}^{+}, \mathrm{PO}_{4}^{3-}$, and dissolved oxygen) (Supplementary Methods S.3.1.5), but did not find any clear differences between HP and LP populations (Online Table S11-S16; Fig. S.3.4). Moreover, when inter-population variation in placentation is directly related to predation risk and the water quality parameters, we still found a higher degree of placentation in HP populations (Supplementary Methods S.3.1.6; Fig. S.3.5). Second, the higher degree of placentation of HP females should not be associated with a difference in maternal traits such as proportion of body fat (BF) and standard length (SL), as they are known to influence the degree of placentation (Hagmayer et al., 2018). This subprediction is met, as the degree of placentation of HP and LP females is predicted for a female of the same SL and BF. In addition, comparisons of maternal traits did not reveal any difference in maternal SL $\left(t_{19.078}=-0.353, P=0.728\right.$; Online Table S17) or BF $\left(t_{5.315}=0.395, P=0.708\right.$; Online Table S18) between HP and LP populations (Fig. S.3.6). Third, the higher degree of placentation of HP females should be due to the production of smaller eggs at fertilization. The locomotor cost hypothesis predicts that an increase in the degree of placentation will convey a potential adaptive benefit to females only if the higher MI is the consequence of producing smaller eggs at fertilization, while offspring size and number at birth, as well as the degree of superfetation remain unchanged. Consistent with these predictions, we found that independent of maternal BF and SL, HP females displayed an average 11\% reduction in egg dry mass at fertilization compared to LP females $\left(t_{109.112}=-3.316\right.$, $P=0.001$; Online Table S2; Fig. 3.2a), while offspring dry mass at birth did not differ 
between HP and LP females $\left(t_{20.752}=-0.253, P=0.802\right.$; Online Table S3; Fig. 3.2b). Likewise, the proportion of egg fat at fertilization $\left(t_{43.513}=-0.386, P=0.702\right.$; Online Table S4; Fig. 3.2c), proportion of offspring fat at birth $\left(t_{20.866}=-0.153, P=0.879\right.$; Online Table S5; Fig. 3.2d), and the degree of superfetation $(z=0.496, P=0.620$; Online Table S6; Fig. 3.2h) did not differ between HP and LP females.
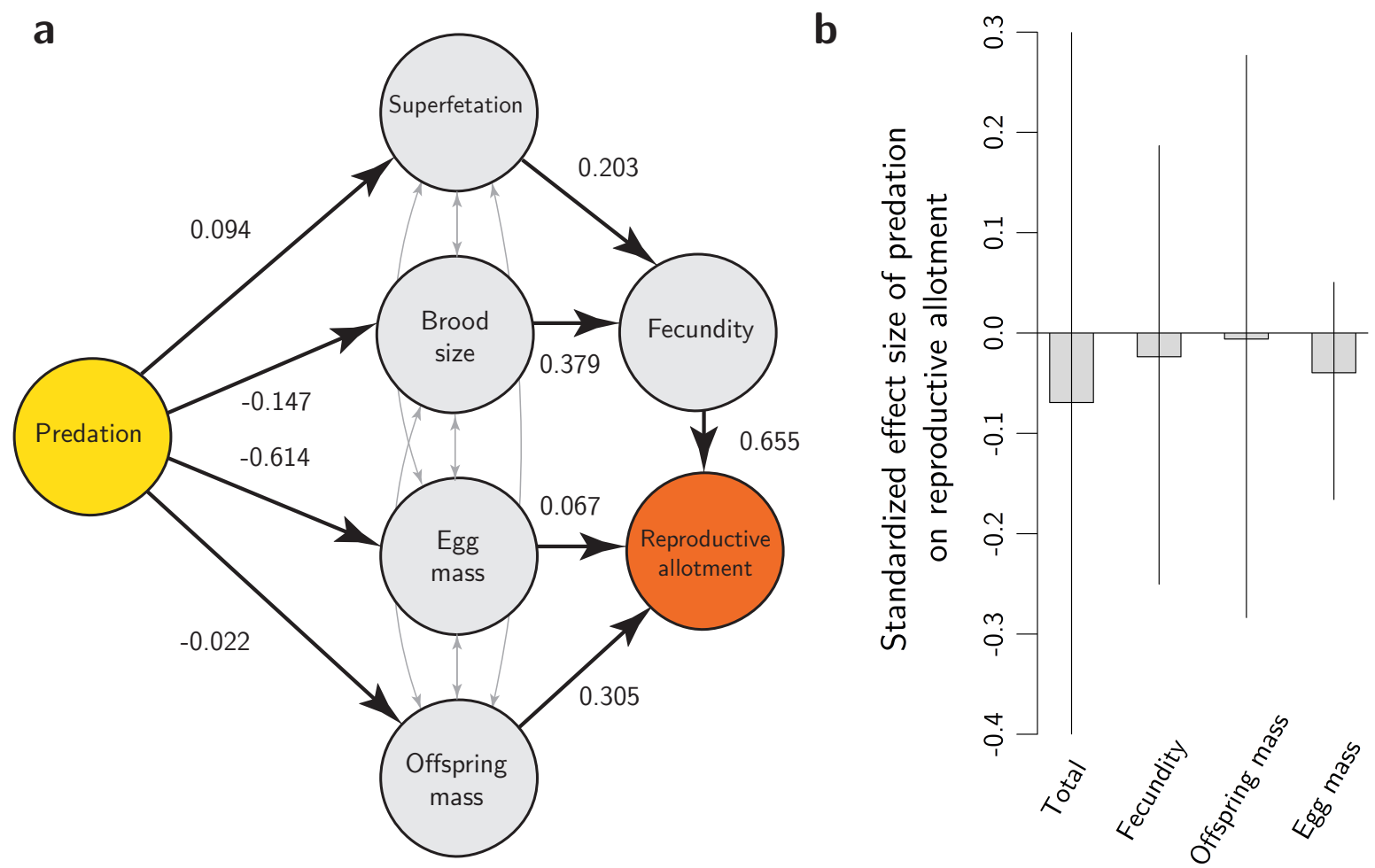

Fig. 3.3. a Graphical illustration of the relationships between predation, egg dry mass at fertilization (i.e. developmental stage 2), offspring dry mass at birth (i.e. developmental stage 45), brood size, fecundity, superfetation, and absolute dry reproductive allotment. The direction of the arrows represents the directionality of the relationships. The numbers equal standardized partial regression coefficients (i.e. the strength of the relationships), which take values between -1 and 1. Grey arrows represent the covariances between the residuals of the responses measured on the same observational unit. b Standardized effect size of predation on the reproductive allotment mediated through the maternal life-history traits $( \pm 95 \% \mathrm{Cl})$. These values equal the contribution of each of the maternal life-history traits to differences in the reproductive allotment among predation regimes. The paths via superfetation and brood size are summed together, as both are mediated via fecundity. Note that $\mathbf{a}$ and $\mathbf{b}$ show that the total effect of predation on the reduction of reproductive allotment is mainly mediated through egg mass at fertilization.

This raises the question how an increase in the degree of placentation in HP populations could confer a potential adaptive advantage to females during pregnancy? If all the above criteria are met, a higher degree of placentation should result in a lower reproductive allotment (RA) during pregnancy, notably without sacrificing fecundity. In line with this, 
we found that HP females had dry RA's that were on average $33 \%$ smaller than those of LP females $\left(t_{21.342}=-3.290, P=0.003\right.$; Online Table S7; Fig. 3.2e), while brood size $(z=-1.074, P=0.283$; Online Table S8; Fig. 3.2f) and fecundity $(z=-0.203$, $P=0.839$; Online Table S9; Fig. 3.2g) did not differ between HP and LP females. Notably, the observed reduction in dry RA by $33 \%$ (17.9 mg) equals the dry mass of 2.6 offspring at birth (stage 45) that are produced by a female of average dry mass (Supplementary Methods S.3.1.7; Online Table S19; Fig. S.3.7). Moreover, we found that the observed maternal traits and life-history pattern in HP females are independent of the type of predator community (Online Table S20-S29; Fig. 3.2, S.3.6). This suggests that different piscivorous predator species drive similar life-history adaptations in P. retropinna.

Finally, the standardized effect sizes $\left(\beta^{*}\right)$ confirm that the reduction in RA in HP females is mainly mediated through egg mass at fertilization $\left(\beta^{*}=-0.040\right)$, rather than offspring mass at birth $\left(\beta^{*}=-0.006\right)$, or brood size, superfetation, and maternal fecundity $\left(\beta^{*}=-0.024\right)$ (Fig. 3.3). In other words, the seemingly small difference in egg mass at fertilization of $11 \%$ largely contributes to the observed difference in RA of $33 \%$. Collectively, these findings show that females from HP localities have a higher degree of placentation and that this significantly reduces their reproductive burden during pregnancy, without negatively affecting their reproductive output.

\subsection{Discussion}

\subsubsection{The degree of placentation in natural populations is shaped by predation}

Comparisons of the degree of placentation among natural populations are of particular interest, because they may provide insights into the evolutionary processes that drive the elaboration of placentas at a macro-evolutionary level (Hansen \& Martins, 1996; Arnold et al., 2001; Reznick \& Ricklefs, 2009; Rolland et al., 2018). To date, few studies have evaluated inter-population variation in the degree of placentation and these did not examine (or used insufficient replicates to make reliable inferences about) the ecological factors that may have driven differences in the degree of placentation among populations (e.g. Schrader \& Travis 2005, compared two populations of the highly placental Heterandria formosa; Pires et al. 2007, two populations of the highly placental Poeciliopsis prolifica; Gorini-Pacheco et al. 2017, three populations of the moderate placental Phalloceros harpagos).

Here, we show that predation risk in streams correlates with the degree of placentation among 28 natural populations of the highly placental $P$. retropinna. Specifically, female $P$. retropinna that co-occur with piscivorous predator species produce smaller eggs at fertilization, while giving birth to an equal number, size, and quality of offspring. The life-history adjustments lead to a significantly higher MI, and hence degree of placentation, in $\mathrm{HP}$ females $\left(\mathrm{MI}_{\mathrm{HP}}=22.86\right)$ compared to LP females $\left(\mathrm{MI}_{\mathrm{LP}}=19.86\right)$.

Our results show that the production of smaller eggs at fertilization, associated with an 
increase in the degree of placentation in HP populations, results in a lower reproductive allotment (RA) for females during pregnancy. Interestingly, comparative studies in the fish families Poeciliidae and Zenarchopteridae show that placentation is consistently associated with a lower RA (Reznick et al., 2007; Bassar et al., 2014). In this study, we show that similar patterns are found at an intra-specific level, among natural populations of a placental species. This suggests that the association between (higher degrees of) placentation and a lower RA may be a general feature of the evolution of placentas, both at a micro- and macro-evolutionary level.

The locomotor cost hypothesis proposes that a lower RA may offer an adaptive benefit to pregnant live-bearing females, because it leads to a more slender body shape and hence improved swimming performance. A number of recent studies seem to support this idea. Fleuren et al. (2018) showed that placental species are more slender at the beginning of pregnancy compared to non-placental ones and that, consistent with the locomotor cost hypothesis (Pollux et al., 2009), this morphological advantage of the placenta diminishes over the course of gestation. Superfetation may amplify this morphological advantage (Pollux et al., 2009) (Fig. S.3.8). A lower RA and streamlined body shape are associated with a reduced drag on the female body (Quicazan-Rubio et al., 2019), higher sustained swimming performance (Plaut, 2002), improved fast-start escape response (Ghalambor et al., 2004; Fleuren et al., 2019), and enhanced survival probability (Walker et al., 2005; Plath et al., 2011; Laidlaw et al., 2014).

The increased degree of placentation and associated decreased RA in HP populations may thus lead to an enhanced predator escape performance. The question is: what is the resulting 'survival benefit' of HP females compared to LP females? In an attempt to quantify the survival advantage of a HP versus a LP female P. retropinna, we used estimates of the effects of body shape on locomotor performance in Poeciliopsis turneri (Fleuren et al., 2019) and the probability of evading a predator strike in Poecilia reticulata (Walker et al., 2005). The difference in wet reproductive allotment between HP and LP female $P$. retropinna is largest just before the parturition of a brood (210.31 mg; Online Table S30; Fig. S.3.8). Relative to body size $\left(0.0013 \mathrm{SL}^{-3}\right)$, this difference is equivalent to an improvement in maximum escape velocity of $1.12 \mathrm{SL} \cdot \mathrm{s}^{-1}$ in $P$. turneri (Fleuren et al., 2019). Based on P. reticulata, such an improvement in escape performance would translate to an increased chance of successfully evading a predator strike of $1.2 \%$ (Walker et al., 2005) (Supplementary Methods S.3.1.8; Fig. S.3.8). At first glance, this may not seem large, however, the strength of selection needed to explain observed rates of evolution can be extremely weak (Lande, 1976). If we assume that, (i) the degree of placentation of HP and LP females represent two different genotypes, (ii) the HP genotype has a survival advantage of $1.2 \%$, (iii) there is no mixing of genotypes, and (iv) the HP genotype begins with a frequency of $1 \%$ in the population, then it only takes 625 generations (or 469 years based on the generation time of 0.75 years in $P$. retropinna) to make up $95 \%$ of the population (Supplementary Methods S.3.1.9; Fig. S.3.9). The maximum time available for the evolution of extensive placentation in the genus Poeciliopsis is estimated 
to be $0.75-2.36$ million years (Reznick et al., 2002). Nevertheless, care must be taken when translating the estimates of the effects of body shape on locomotor performance and survival probability based on other poeciliid species to P. retropinna. Although the relationship between body shape and escape performance was shown to not differ between three different placental poeciliid species (Fleuren et al., 2019), the relationship between locomotor performance and survival probability is based on P. reticulata. Compared to $P$. retropinna, $P$. reticulata is non-placental without superfetation which may affect the association with survival also other than via improving locomotor performance (e.g. behavioral differences).

To be considered an adaptation, differences in placentation among HP and LP populations must be heritable, rather than entirely phenotypically plastic. Turcotte et al. (2008) have investigated the patterns of maternal provisioning in naturally occurring hybrids between Poeciliopsis monacha, a lecithotrophic species that produces large eggs at fertilization, and P. lucida, a moderately matrotrophic species that produces small eggs and provisions embryos with nutrients throughout gestation. Hybrids produced intermediate-sized eggs, suggesting that egg mass at fertilization is to some degree genetically controlled. Given that egg mass is heritable and subject to selection, the observed differences in egg mass at fertilization in response to predation risk are thus likely to be evolved.

In conclusion, our results provide the first compelling evidence in support of the locomotor cost hypothesis by showing that an increase in the degree of placentation can offer a selective advantage in high 'performance-demanding' (i.e. high-predation localities) environments (Pollux et al., 2009; Pires et al., 2011).

\subsubsection{No support for the resource allocation hypothesis}

The resource allocation hypothesis proposes that the placenta evolved because the associated reduction in egg size at fertilization allows females to attain higher fitness through increased brood sizes. Trexler \& DeAngelis (2003) argued that the ability to selectively abort embryos and recycle this investment, should resource conditions suddenly deteriorate, is a crucial preadaptation for the evolution of placentation and that female fat reserves might serve to buffer placental species from fluctuating resource conditions.

Two findings appear to tentatively argue against this hypothesis. First, prior studies in four independent placental lineages have shown that poeciliid fish are not able to abort embryos in response to sudden adverse resource conditions (Reznick et al., 1996b; Banet \& Reznick, 2008; Banet et al., 2010; Pollux \& Reznick, 2011). Second, if female fat storage serves to buffer placental species from fluctuating resource conditions, then abortion incidence should increase when food conditions become unfavorable and maternal fat reserves insufficient to fully buffer females during gestation. In other words, betterconditioned females (those carrying larger fat reserves) should be less likely to abort embryos. Contrary to this expectation, we found an increase in the abortion incidence with a higher proportion of maternal body fat: better-conditioned females were more 
likely to abort embryos $\left(t_{230.335}=2.186, P=0.03\right.$; Online Table S10; Fig. S.3.2). The mechanisms behind this higher rate of embryo abortion in better-conditioned female $P$. retropinna are currently unclear and require further investigation.

\subsubsection{Conclusion: placentation facilitates the evolution of new life-history opportunities}

To date, the effect of predation risk on poeciliid life-histories has almost exclusively been studied in non-placental species including Poecilia reticulata (Reznick \& Endler, 1982; Reznick et al., 1990; Reznick \& Bryga, 1996), Brachyrhaphis rhabdophora (Johnson \& Belk, 2001), Brachyrhaphis episcopi (Jennions et al., 2006), Gambusia hubbsi (Downhower et al., 2000), and Xiphophorus hellerii (Basolo \& Wagner, 2004). As predicted by theoretical studies (Gadgil \& Bossert, 1970; Law, 1979; Michod, 1979), guppies adaptively respond to increased adult mortality by devoting a larger percentage of their body mass to their developing offspring (Reznick \& Endler, 1982; Reznick et al., 1990). Furthermore, they exhibit shorter time intervals between successive broods and produce more, but smaller, offspring (Reznick, 1982b,a, 1983; Reznick \& Endler, 1982; Reznick et al., 1990). These predation-driven life-history differences were shown to have a genetic basis (Reznick, 1982b). Smaller offspring, however, have a lower ability to capture prey (Lankheet et al., 2016), compete for food (Bashey, 2006), escape predators (Gibb et al., 2006), and hence have a lower survival probability in low food and/or high predation environments (Bashey, 2006; Dial et al., 2016). Thus, it has been argued that selection for increased maternal fecundity and smaller offspring in Trinidadian guppies indicates that maternal fitness, rather than offspring fitness, may have dominated in shaping the evolution of offspring size in response to predation (Dial et al., 2016).

Our study reveals that matrotrophic species may be able to adopt strikingly different solutions to deal with high predation, solutions that are not possible in lecithotrophic species such as the guppy. In high predation environments, females of the placental P. retropinna reduced their reproductive allotment, notably without reducing the size or number of offspring at birth. This is likely to be advantageous for the mother, as the decreased reproductive allotment improves her locomotor performance (Plaut, 2002; Ghalambor et al., 2004; Fleuren et al., 2019; Quicazan-Rubio et al., 2019) and survival probability in high predation environments (Plath et al., 2011; Laidlaw et al., 2014). This life-history adaptation is however unavailable to lecithotrophic species. In theory, lecithotrophic species could respond to high predation risk by (i) reducing the reproductive allotment at the expense of either fecundity or offspring size, or (ii) increasing fecundity at the expense of maternal mortality. Studies have shown that lecithotrophic species principally do the latter (Reznick \& Endler, 1982; Reznick et al., 1990; Reznick \& Bryga, 1996; Downhower et al., 2000; Johnson \& Belk, 2001; Basolo \& Wagner, 2004; Jennions et al., 2006). The evolution of the placenta opens up novel opportunities for females to respond to high predation risk that are not available to lecithotrophic species. Specifically, placental females are able to reduce their reproductive allotment seemingly without any reproductive cost; 
i.e. without sacrificing either fecundity or offspring quality at birth.

\section{S.3 Supplementary Information}

Additional supplementary information can be found in the online version of the article: https://onlinelibrary.wiley.com/doi/full/10.1111/ele.13487

\section{S.3.1 Supplementary Methods}

\section{S.3.1.1 Laboratory measurements}

The fish samples were transported to the Reznick lab (University of California Riverside, USA) and Pollux lab (Wageningen University, The Netherlands) for anatomical dissections. The standard length of preserved specimens was measured to the nearest $\mathrm{mm}$ from the tip of the upper jaw to the outer margin of the hypural plate, using a caliper. Female dry mass was measured to the nearest $0.01 \mathrm{mg}$ on a Mettler Toledo AE163 Microbalance (specimens collected in 2013) or Mettler Toledo XP205 delta range (specimens collected in 2017 and 2018) (Mettler Instruments Corp., Hightstown, New Jersey, USA) after removing the ovary and air-drying the female overnight at $60^{\circ} \mathrm{C}$ in a drying oven. Female lean mass was measured by extracting the fat twice with anhydrous diethyl ether (Fisher Scientific) to remove triglycerides, and by subsequently air-drying and re-weighting the female (see above). The proportion of maternal body fat was then calculated by subtracting maternal lean mass from maternal dry mass divided by maternal dry mass. The embryo dry mass for a given brood was calculated as the dry mass of the brood, measured to the nearest $0.01 \mathrm{mg}$ after air-drying overnight at $60^{\circ} \mathrm{C}$ (see above), divided by the number of embryos in the brood (Pollux \& Reznick, 2011).

\section{S.3.1.2 Population-specific Matrotrophy Indices}

Population-specific Matrotrophy Indices (MI's) were estimated using the Bayesian programming environment JAGS (Plummer, 2003). For this, ln-transformed embryo dry mass was fitted in a linear model as a function of the developmental stage of embryos (second order polynomial), the proportion of maternal body fat, maternal standard length, and the interaction between the developmental stage of embryos and the proportion of maternal body fat. For each population, the model estimates year-specific intercepts and year-specific slopes on the developmental stage of the embryos (second order polynomial). This allows to predict the MI for a given population in a specific year. In addition, the model includes the mother identity as additional intercept to account for variation among females that is not accounted by maternal body fat and standard length. We used $N\left(0,5^{2}\right)$ priors for the proportion of maternal body fat, maternal standard length, and the interaction between the developmental stage of embryos and the proportion of maternal body fat. For mother identity, we used a $N\left(0, \sigma^{2}\right)$ prior with the common variance $\sigma^{2} \sim$ Inv-Gamma $(0.01,0.01)$, parameterized with shape and rate parameters. The 
year-specific intercepts and year-specific slopes on the developmental stage of the embryos (second order polynomial) were estimated using $N\left(\mu, \sigma^{2}\right)$ priors, with the common mean $\mu \sim N\left(0,2^{2}\right)$ and the common standard deviation $\sigma \sim$ Student- $_{(0, \infty)}\left(0,1^{2}, 1\right)$, parameterized with mean, variance, and degrees of freedom.

The population-specific MI's were subsequently calculated by dividing offspring dry mass at birth (developmental stage 45) by egg dry mass at fertilization (developmental stage 2) that were predicted for a given population in a specific year and for a female of overall average standard length (i.e. $\bar{y}_{\text {standard length }}=53 \mathrm{~mm}$ ) and proportion of body fat (i.e. $\left.\bar{y}_{\text {body fat }}=0.16\right)$. Since all population-specific MI's are predicted for a female of the same length and body fat, the resulting MI's are independent of these traits (Fig. S.3.1). However, the 'raw' MI for each population in a specific year (i.e. predicted based on the observed, rather than the average maternal body fat and standard length) is also provided (Table S.3.1).

Three MCMC chains were run for 1,500,000 iterations, with a burnin of 500,000 and a thinning of 1000. Convergence was assessed by visual examination of the traces and by checking that $\hat{R}<1.01$. The model fit was assessed using a posterior predictive check on the predictions of embryo mass.

$$
\begin{aligned}
\log \left(\overline{\mathrm{EM}}_{i, j}\right) \sim & N\left(\alpha_{\text {pop } \times \text { year }}+\alpha_{\text {mother }}+\right. \\
& \beta_{1, \text { pop } \times \text { yearstage }}+\beta_{2, j}+\beta_{2 \text { pop } \times \text { yearstage }}^{2}+, \\
& \left.\beta_{3} \mathrm{mBF}_{i}+\beta_{4} \mathrm{mSL}_{i}+\beta_{5} \mathrm{mBF}_{i} \times \text { stage }_{i, j}, \sigma^{2}\right),
\end{aligned}
$$

where $\log \left(\overline{\mathrm{EM}}_{i, j}\right)$ corresponds to the ln-transformed mean embryo dry mass of the $j$ th brood carried by the $i$ th female. $\alpha_{\text {pop } \times \text { year }}$ corresponds to the year-specific intercept of a given population and $\alpha_{\text {mother }}$ to the mother identity. $\beta_{1, \text { pop } \times \text { yearstage }}{ }_{j, i}$ is the year-specific slope of a given population on the developmental stage of the $j$ th brood carried by the $i$ th female (second order polynomial), $\mathrm{mBF}_{i}$ the arcsin square-root transformed proportion of body fat of the $i$ th female, $\mathrm{mSL}_{i}$ the standard length of the $i$ th female, and $\sigma$ the residual standard deviation.

\section{S.3.1.3 Predation-specific Matrotrophy Indices}

Predation-specific Matrotrophy Indices (MI's) were estimated using the Bayesian programming environment JAGS (Plummer, 2003). For this, ln-transformed embryo dry mass was fitted in a linear model as a function of the developmental stage of embryos (second order polynomial), high and low predation risk (i.e. piscivorous predator species present or absent), the proportion of maternal body fat, maternal standard length, the interaction between the developmental stage of embryos and the proportion of maternal body fat, and the interaction between the developmental stage of embryos and predation risk. For each population, the model estimates an additional year-specific intercept to correct for differences between populations in a specific year. Moreover, the model includes the mother identity as additional intercept to account for variation among females that is 
not accounted by maternal body fat and standard length. We used $N\left(0,5^{2}\right)$ priors for all fixed effects. For mother identity, we used a $N\left(0, \sigma^{2}\right)$ prior with the common variance $\sigma^{2} \sim \operatorname{Inv}$-Gamma $(0.01,0.01)$, parameterized with shape and rate parameters. The year-specific intercepts and year-specific slopes on the developmental stage of the embryos (second order polynomial) were estimated using $N\left(\mu, \sigma^{2}\right)$ priors, with the common mean $\mu \sim N\left(0,1^{2}\right)$ and the common standard deviation $\sigma \sim$ Student- $t_{(0, \infty)}\left(0,1^{2}, 1\right)$, parameterized with mean, variance, and degrees of freedom.

The MI for high and low predation females was subsequently calculated by dividing offspring dry mass at birth (developmental stage 45) by egg dry mass at fertilization (developmental stage 2) that were predicted for a given predation regime (i.e. piscivorous predator species present or absent) and for a female of overall average standard length (i.e. $\bar{y}_{\text {standard length }}=53 \mathrm{~mm}$ ) and proportion of body fat (i.e. $\bar{y}_{\text {body fat }}=0.16$ ).

Three MCMC chains were run for 1,500,000 iterations, with a burnin of 500,000 and a thinning of 1000. Convergence was assessed by visual examination of the traces and by checking that $\hat{R}<1.01$. The model fit was assessed using a posterior predictive check on the predictions of embryo mass.

$$
\begin{aligned}
\log \left(\overline{\mathrm{EM}}_{i, j}\right) \sim & N\left(\alpha_{\text {pop } \times \text { year }}+\alpha_{\text {mother }}+\right. \\
\text { intercept }+ & \beta_{1} \text { stage }_{i, j}+\beta_{2} \text { stage }_{i, j}^{2}+\beta_{3} \text { predation }_{i}+ \\
& \beta_{4} \mathrm{mBF}_{i}+\beta_{5} \mathrm{mSL}_{i}+\beta_{6} \mathrm{mBF}_{i} \times \text { stage }_{i, j}+ \\
& \left.\beta_{7} \text { predation }_{i} \times \text { stage }_{i, j}, \sigma^{2}\right),
\end{aligned}
$$

where $\log \left(\overline{\mathrm{EM}}_{i, j}\right)$ corresponds to the ln-transformed mean embryo dry mass of the $j$ th brood carried by the $i$ th female. $\alpha_{\text {pop } \times \text { year }}$ corresponds to the year-specific intercept of a given population and $\alpha_{\text {mother }}$ to the mother identity. intercept is the overall intercept, $\beta_{1}$ stage $_{j, i}$ the developmental stage of the $j$ th brood carried by the $i$ th female (second order polynomial), predation $_{i}$ the predation risk experienced by the $i$ th female, $\mathrm{mBF}_{i}$ the arcsin square-root transformed proportion of body fat of the $i$ th female, $\mathrm{mSL}_{i}$ the standard length of the $i$ th female, and $\sigma$ the residual standard deviation.

\section{S.3.1.4 Path analysis}

Differences in reproductive allotment among populations could be due to effects on several life-history traits. For instance, reproductive allotment could be decreased by reducing brood size or superfetation, which in turn decreases the number of embryos (i.e. fecundity). Alternatively, reproductive allotment diminishes when producing smaller eggs at fertilization or offspring at birth. We used a path analysis to determine the contribution of each of these life-history traits to differences in reproductive allotment among predation regimes. In total, three (generalized) linear mixed effects models implemented in MCMCglmm (Hadfield, 2010) were used to estimate all paths.

In the first model, we estimated the direct effect of predation risk on $z$-standardized egg dry mass at fertilization (developmental stage 2) (ze), z-standardized offspring dry mass 
at birth (developmental stage 45) (zo), z-standardized average brood size for a given mother $(z b)$, and the degree of superfetation $(s)$ in a multivariate model:

$$
[z e, z o, z b, s] \sim \mu+\mathbf{X} \beta+\mathbf{Z} r
$$

where $\mu$ is the intercept vector, $\beta$ the vector of fixed effects, $\mathbf{X}$ the corresponding design matrix, and $\mathbf{Z}$ is a design matrix for additional random terms $r$. $z$-standardized egg dry mass at fertilization, offspring dry mass at birth, and the average brood size for a given mother are assumed to be Gaussian-distributed. Superfetation was formulated in a generalized linear mixed model framework using a log link for the Poisson-distributed response. Fixed effects include predation risk (i.e. piscivorous predator species present or absent), $z$-standardized proportion of maternal body fat, $z$-standardized maternal standard length, and the $z$-standardized developmental stage of the most-developed brood in the case of superfetation. Besides an error, the random terms include population and river identity accounting for spatio-temporal non-independence of observations. Moreover, the interaction between population identity and year quantifies variation of a given population between years. The multivariate framework allows for the covariance between the residuals of all responses. As priors, we used the default normal priors for the fixed effects with the expected value of 0 and variance $10^{12}$, and inverse-Wishart priors for the variances with the expected value of 1 and degree of belief of 3.002. The number of iterations was 5,500,000, with a burnin of 500,000 and a thinning of 5000 .

In the second model, maternal fecundity was fitted as a function of superfetation and average brood size for a given mother, as changes in both brood size and superfetation will affect the number of embryos:

$$
\begin{aligned}
f_{i} \sim & P o\left(\lambda_{i}\right), \log \left(\lambda_{i}\right)=\alpha+\alpha_{\text {pop }}+\alpha_{\text {river }}+\alpha_{\text {pop } \times \text { year }}+ \\
& \beta_{1} z s_{i}+\beta_{2} z b_{i}+\beta_{3} \mathrm{mBF}_{i}+\beta_{4} \mathrm{mBF}_{i}^{2}+\beta_{5} \mathrm{mSL}_{i}+\beta_{6} \text { latestStage }_{i}
\end{aligned}
$$

where $f_{i}$ corresponds to the fecundity (i.e. number embryos) of the $i$ th female. $\alpha$ corresponds to the overall intercept, and $\alpha_{\text {pop }}, \alpha_{\text {river }}$, and $\alpha_{\text {pop } \times \text { year }}$ to the random intercepts. $z s_{i}$ is the $z$-standardized degree of superfetation, $z b_{i}$ the $z$-standardized average brood size, $z \mathrm{mBF}_{i}$ the $z$-standardized proportion of maternal body fat (second order polynomial), $z \mathrm{mSL}_{i}$ the $z$-standardized maternal standard length, and $z$ latestStage ${ }_{i}$ the $z$-standardized developmental stage of the most developed brood of the $i$ th female. As priors, we used the default normal priors for the fixed effects with the expected value of 0 and variance $10^{12}$, and inverse-Wishart priors for the variances with the expected value of 1 and degree of belief of 0.002 . The number of iterations was 1,500,000, with a burnin of 500,000 and a thinning of 1000 .

The third model subsequently predicts the absolute dry reproductive allotment as a function of fecundity, egg dry mass at fertilization, and offspring dry mass at birth: 


$$
\begin{aligned}
z \mathrm{ra}_{i} \sim & N\left(\alpha+\alpha_{\text {pop }}+\alpha_{\text {river }}+\alpha_{\text {pop } \times \text { year }}+\right. \\
& \left.\beta_{1} z f_{i}+\beta_{2} z e_{i}+\beta_{3} z o_{i}+\beta_{4} z \mathrm{mBF}_{i}+\beta_{5} z \mathrm{mSL}_{i}, \sigma^{2}\right),
\end{aligned}
$$

where $z \mathrm{ra}_{i}$ corresponds to the absolute dry reproductive allotment of the $i$ th female. $\alpha$ corresponds to the overall intercept, and $\alpha_{\text {pop }}, \alpha_{\text {river }}$, and $\alpha_{\text {pop } \times \text { year }}$ to the random intercepts. $z f_{i}$ is the $z$-standardized maternal fecundity (i.e. number embryos), $z e_{i}$ the $z$-standardized egg dry mass at fertilization (developmental stage 2), $z o_{i}$ the $z$-standardized offspring dry mass at birth (developmental stage 45 ), $z \mathrm{mBF}_{i}$ the $z$-standardized proportion of maternal body fat, $z \mathrm{mSL}_{i}$ the $z$-standardized maternal standard length of the $i$ th female, and $\sigma$ the residual standard deviation. As priors, we used the default normal priors for the fixed effects with the expected value of 0 and variance $10^{12}$, and inverse-Wishart priors for the variances with the expected value of 1 and degree of belief of 0.002 . The number of iterations was 1,500,000, with a burnin of 500,000 and a thinning of 1000 .

For all models, convergence was assessed by visual examination of the traces and by checking that the autocorrelations of the parameter chain was less than 0.1. In addition, each model was re-fitted as a function of an intercept only (null model) to compare the deviance information criterion (DIC) of the full model against the DIC of the null model $(\Delta \mathrm{DIC})$.

\section{S.3.1.5 Measurements of water parameters}

The water velocity was measured at each location to the nearest $0.01 \mathrm{~m} \cdot \mathrm{s}^{-1}$ with a Höntzsch Vane Wheel FA current meter (type ZS30 GFE md20 T/100-2/p10, Höntzsch Instruments, Waiblingen, Germany). Depending on the uniformity of the flow, the water velocity was taken at 9-17 incremental observation points across a transect of the stream. At each observation point, the mean water velocity was defined as the average of three repeated measurements at a height above the stream bed equal to 0.4 times the depth at that location. When the water depth exceeded $60 \mathrm{~cm}$, the mean water velocity was calculated as the average between the velocities measured at 0.2 times the water depth and 0.8 times the water depth (Hauer \& Lamberti, 2007). Each location was additionally characterized by measuring salinity (S) by using the ExStik II pH/conductivity/TDS meter (Extech Instruments, Nashua, USA), hardness $\left(\mathrm{mg} \cdot \mathrm{L}^{-1}\right)$ with a titrimetric color-test kit (Merck KGaA, Darmstadt, Germany), ammonium concentration $\left(\mathrm{NH}_{4}^{+}\right)\left(\mathrm{mg} \cdot \mathrm{L}^{-1}\right)$ as a proxy for the nitrogen loading of a stream by using a colorimetric ammonium test (Merck KGaA, Darmstadt, Germany), phosphate concentration $\left(\mathrm{PO}_{4}^{3-}\right)\left(\mathrm{mg} \cdot \mathrm{L}^{-1}\right)$, as phosphorus is an important determinant of primary production in freshwater ecosystems (Schindler, 1977) by using a colorimetric phosphate test (Merck KGaA, Darmstadt, Germany), and dissolved oxygen (\%) with the ExStik DO600 meter (Extech Instruments, Nashua, USA). Salinity and dissolved oxygen were measured 1-3 times at each location, whereas hardness, ammonium concentration, and phosphate concentration were measured only once. 
Salinity was then predicted for each location at $25^{\circ} \mathrm{C}$ in the following linear model by Maximum Likelihood:

$$
\mathrm{S}_{i, j} \sim N\left(\alpha+\beta_{1} \operatorname{location}_{i}+\beta_{2} \text { temperature }_{i, j}, \sigma^{2}\right),
$$

where $\mathrm{S}_{i, j}$ corresponds to the $j$ th measurement of salinity at location $i, \alpha$ to the overall intercept, location $_{i}$ to the $i$ th location, temperature $i_{i, j}$ to the $j$ th measurement of water temperature at location $i$, and $\sigma$ to the residual standard deviation.

Dissolved oxygen was predicted for each location at 13:00 hours and $25^{\circ} \mathrm{C}$ in the following linear model by Maximum Likelihood:

$$
\text { oxygen }_{i, j} \sim N\left(\alpha+\beta_{1} \text { location }_{i}+\beta_{2} \text { temperature }_{i, j}+\beta_{3} \text { time }_{i, j}+\beta_{4} \text { time }_{i, j}^{2}, \sigma^{2}\right),
$$

where oxygen ${ }_{i, j}$ corresponds to the $j$ th measurement of dissolved oxygen at location $i, \alpha$ to the overall intercept, location $_{i}$ to the $i$ th location, temperature ${ }_{i, j}$ to the $j$ th measurement of water temperature at location $i$, time $i, j$ to the $z$-standardized numeric day time of the $j$ th measurement of dissolved oxygen at location $i$, and $\sigma$ to the residual standard deviation.

\section{S.3.1.6 Relationship between population-specific Matrotrophy Indices and environmental variables}

Either by influencing egg mass at fertilization or offspring mass at birth, many populationspecific factors are likely to contribute to the observed inter-population variation in the degree of placentation. To quantify the potential effects of additional water parameters at each location on the degree of placentation, the population-specific Matrotrophy Indices (MI's) estimated in the model described in Equation S.3.1 were fitted as a function of all measured environmental variables (predation risk, salinity, water velocity, hardness, $\mathrm{NH}_{4}^{+}, \mathrm{PO}_{4}^{3-}$, and dissolved oxygen) in a linear mixed effects model using Restricted Maximum Likelihood. The continuous predictors were $z$-standardized in order to make them comparable (Schielzeth, 2010). Moreover, the model includes river identity as additional intercept to account for variation among rivers that is not accounted by the measured environmental parameters.

$$
\begin{aligned}
z \mathrm{MI}_{i} \sim & N\left(\alpha_{\text {river }}+\text { intercept }+\right. \\
& \beta_{1} \text { predation }_{i}+\beta_{2} z \text { salinity }_{i}+\beta_{3} z \text { hardness }_{i}+ \\
& \left.\beta_{4} z \text { water velocity }_{i}+\beta_{5} z \mathrm{NH}_{4, i}^{+}+\beta_{6} z \mathrm{PO}_{4, i}^{3-}+\beta_{7} z \mathrm{O}_{2, i}, \sigma^{2}\right),
\end{aligned}
$$

where $z \mathrm{MI}_{i}$ corresponds to the Matrotrophy Index for the $i$ th population in a specific year estimated in the model described in Equation S.3.1. $\alpha_{\text {river }}$ corresponds to the riverspecific intercept and intercept to the the overall intercept. predation ${ }_{i}$ is the predation 
risk (i.e. piscivorous predator species present or absent), $z_{\text {salinity }}$ the $z$-standardized water salinity $(\mathrm{S})$, zhardness ${ }_{i}$ the $z$-standardized hardness $\left(\mathrm{mg} \cdot \mathrm{L}^{-1}\right)$, zwater velocity ${ }_{i}$ the $z$-standardized mean water velocity $\left(\mathrm{m} \cdot \mathrm{s}^{-1}\right), z \mathrm{NH}_{4, i}^{+}$the $z$-standardized ammonium concentration $\left(\mathrm{mg} \cdot \mathrm{L}^{-1}\right), z \mathrm{PO}_{4, i}^{3-}$ the $z$-standardized phosphate concentration $\left(\mathrm{mg} \cdot \mathrm{L}^{-1}\right), z \mathrm{O}_{2, i}$ the $z$-standardized dissolved oxygen (\%), and $\sigma$ the residual standard deviation.

\section{S.3.1.7 Embryo growth during gestation}

Embryo growth during gestation was estimated as the exponential relationship between embryo dry mass and the developmental stage of embryos (second order polynomial) in a linear mixed effects model by Restricted Maximum Likelihood (Hagmayer et al., 2018). The model included maternal dry mass, and the interaction between the developmental stage of embryos and maternal dry mass as additional fixed effects (Fig. S.3.7). Mother identity was fitted as random intercept to correct for pseudo-replication. Population, year, and river identity were fitted as random intercepts accounting for spatio-temporal non-independence of observations. Moreover, the interaction between population identity and year was fitted as random intercept to quantify the variation of a given population between years:

$$
\begin{aligned}
\log \left(\overline{\mathrm{EM}}_{i, j}\right) \sim & N\left(\alpha+\alpha_{\text {mother }}+\alpha_{\text {pop }}+\alpha_{\text {year }}+\alpha_{\text {river }}+\alpha_{\text {pop } \times \text { year }}+\right. \\
& \left.\beta_{1} \text { stage }_{i, j}+\beta_{2} \text { stage }_{i, j}^{2}+\beta_{3} \mathrm{mM}_{i}+\beta_{4} \mathrm{mM}_{i} \times \text { stage }_{i, j}, \sigma^{2}\right),
\end{aligned}
$$

where $\log \left(\overline{\mathrm{EM}}_{i, j}\right)$ corresponds to the ln-transformed mean embryo dry mass of the $j$ th brood carried by the $i$ th female. $\alpha$ corresponds to the overall intercept, $\alpha_{\text {mother }}, \alpha_{\text {pop }}$, $\alpha_{\text {year }}, \alpha_{\text {river }}$, and $\alpha_{\text {pop } \times \text { year }}$ to the random intercepts. stage $_{i, j}$ is the developmental stage of the $j$ th brood carried by the $i$ th female, $\mathrm{mM}_{i}$ the dry mass of the $i$ th female, and $\sigma$ the residual standard deviation.

\section{S.3.1.8 Relationship between reproductive allotment, locomotor performance, and survival probability}

Fleuren et al. (2019) studied the locomotor performance in three placental live-bearing fish species (family Poeciliidae) that exhibit different levels of superfetation. Particularly, they used computer-vision based techniques to study changes in body shape (e.g. volume) and three-dimensional fast-start escape performance (e.g. maximum escape velocity) during pregnancy in Poeciliopsis turneri, Heterandria formosa, and Phalloptychus januarius. The slope between body shape and escape performance was not significantly different between the three species. Here we used the relationship between female volume and maximum escape velocity in Poeciliopsis turneri to predict the maximum escape velocity in Poeciliopsis retropinna. P. retropinna and P. turneri are similar regarding their degree of post-fertilization maternal provisioning and degree of superfetation. We derived female volume from female wet mass, assuming an uniform tissue density $(\rho)$ of $1 \mathrm{~g} \cdot \mathrm{cm}^{-3}$ 
(Robertson et al., 2008). The relationship between female volume and maximum escape velocity is then given by:

$$
\hat{v}_{\max }=\alpha+\beta_{1} \frac{m}{\rho \mathrm{SL}^{3}}+\beta_{2} r_{\max }
$$

where $\hat{v}_{\max }$ corresponds to the normalized maximum escape velocity (normalized for standard length SL), $\alpha$ to the overall intercept, and $\frac{m}{\rho \mathrm{SL}^{3}}$ to the normalized female volume (normalized for standard length $\mathrm{SL}^{3}$ ) derived from the tissue density $\rho$, female wet mass $m$, and the average standard length of a pregnant $P$. retropinna female. $r_{\max }$ is the maximum caudal peduncle curvature rate in the kinematic stage 2 of the fast-start escape response.

The estimated maximum escape velocity for a given wet mass was then used to predict the probability of evading the strike of a natural predator. Based on a study by Walker et al. (2005) with Guppies (Poecilia reticulata), the probability of evading a predator $(\omega)$ was predicted using four parameters: (i) the initial distance between predator and prey $\left(D_{\text {pred }}\right)$, (ii) the average speed of the predator $\left(\bar{\nu}_{\text {pred }}\right)$, (iii) the evasion path of the prey relative to the strike path of the predator $\left(\theta_{\text {pred }}\right)$, and (iv) the maximum velocity of the prey $\left(v_{\max }=\hat{v}_{\max } \cdot \mathrm{SL}_{\text {Guppy }}\right)$ estimated in Equation S.3.10:

$$
\operatorname{logit}(\omega)=\alpha+\beta_{1} v_{\max }+\beta_{2} \theta_{\text {pred }}+\beta_{3} \bar{\nu}_{\text {pred }}+\beta_{4} D_{\text {pred }}
$$

\section{S.3.1.9 Simulating selection}

To illustrate that the strength of selection needed to explain observed rates of evolution, assuming that genetic drift is not involved, can be extremely weak, we have simulated the frequency $p^{2}$ of a high predation genotype $\left(A_{1} A_{1}\right)$, and the complementary frequency $q^{2}$ of a low predation genotype $\left(A_{2} A_{2}\right)$ over time (i.e. generations). The HP genotype is assumed to have a survival advantage $(s)$ of $1.2 \%$, and there is no mixing of the genotypes. The initial frequency $\left(p_{0}^{2}\right)$ of the advantageous genotype in the population is assumed to be 0.01 .

The initial genotype frequencies are given by:

$$
p_{0}^{2}, \quad \text { and } \quad q_{0}^{2}=1-p_{0}^{2}
$$

The fitness $\omega$ of the genotypes $A_{1} A_{1}$ and $A_{2} A_{2}$ are given by:

$$
\omega_{11}=1, \quad \text { and } \quad \omega_{22}=1-s
$$

The mean fitness $\varpi$ of all the individuals in the population at time $t$ is then given by:

$$
\varpi_{t}=p_{t}^{2} \omega_{11}+q_{t}^{2} \omega_{22}
$$


The genotype frequencies after selection can then be calculated by:

$$
p_{t}^{\prime 2}=\frac{p_{t}^{2} \omega_{11}}{\varpi_{t}}, \quad \text { and } \quad q_{t}^{\prime 2}=\frac{q_{t}^{2} \omega_{22}}{\varpi_{t}}
$$

\section{S.3.2 Supplementary Results}

\section{S.3.2.1 Relationship between maternal traits and life-history}

Both the proportion of maternal body fat and standard length are significantly associated with maternal fecundity. The correlation between maternal fecundity and standard length is strongly positive $(z=16.993, P<0.001$; Fig. S.3.2). The linear and quadratic relationship between fecundity and body fat are both negative (linear: $z=-5.506, P<0.001$; Fig. S.3.2; quadratic: $z=-4.474, P<0.001)$. Similarly, brood size shows a negative correlation with proportion of maternal body fat $(z=-4.701, P<0.001$; Fig. S.3.2) and a strong positive correlation with standard length $(z=15.713, P<0.001$; Fig. S.3.2). By contrast, the degree of superfetation is not significantly correlated with the proportion of maternal body fat $(z=-1.712, P=0.087$; Fig. S.3.2) or standard length $(z=-0.483$, $P=0.629$; Fig. S.3.2). In addition, egg dry mass at fertilization (mg) increases as a function of increased maternal standard length $\left(t_{117.432}=4.008, P<0.001\right.$; Fig. S.3.2), but is not correlated with the proportion of maternal body fat $\left(t_{116.329}=-1.270, P=0.207\right.$; Fig. S.3.2). Offspring dry mass at birth $(\mathrm{mg})$ is positively associated with the proportion of maternal body fat $\left(t_{79.502}=4.208, P<0.001\right.$; Fig. S.3.2) and standard length $\left(t_{132.520}=2.191, P=0.030 ;\right.$ Fig. S.3.2). Abortion incidence shows a negative linear $\left(t_{213.123}=-3.615, P<0.001 ;\right.$ Fig. S.3.2 $)$ and a positive quadratic association with standard length $\left(t_{391.638}=2.873, P=0.004\right.$; Fig. S.3.2). Moreover, abortion incidence is positively correlated with the proportion of maternal body fat $\left(t_{230.335}=2.186, P=0.03\right.$; Fig. S.3.2).

\section{S.3.3 Supplementary Discussion}

\section{S.3.3.1 Relationship between maternal traits and life-history}

Maternal traits can profoundly influence the offspring phenotype and maternal life-history (Mousseau \& Fox, 1998b). Consistent with previous findings in P. retropinna (Hagmayer et al., 2018), larger females have greater fecundity, produce larger eggs at fertilization, and offspring at birth (Fig. S.3.2). Furthermore, females that have more fat reserves produce fewer but larger offspring at birth, without investing more in egg size at fertilization. The negative correlation between the proportion of maternal body fat and fecundity may reflect a trade-off between offspring size and number; the production of large offspring may necessarily entail the production of fewer offspring owing to the limited size of the female body cavity (Stearns, 1992). The potential mechanisms behind the increased postfertilization maternal provisioning by larger and better-conditioned females, however, is unclear (Hagmayer et al., 2018). By contrast, maternal fecundity is proposed to increase 
with female size, as a consequence of more space available in the female's body cavity (Shine, 1992). The physical constraint of the body cavity is also displayed by the populations where $P$. retropinna co-occur with $P$. dovii as the only predator species. Independently of the proportion of maternal body fat and standard length, these females produce very large offspring at birth (Fig. 3.2), but carry significantly fewer broods at different developmental stages (i.e. superfetation; Fig. 3.2). These females were mainly collected from a single stream (Rio Sucio) with exceptional high nitrogen loading $\left(\mathrm{NH}_{4}^{+}\right.$ concentration measured in $20172.71 \times$ larger than average), and probably nutrient-rich water. As a result, the females are very large (Fig. S.3.6), contain a large amount of body fat (Fig. S.3.6), and invest more in offspring size at birth (Fig. 3.2). Here, we additionally show that the degree of superfetation does not correlate with the proportion of maternal body fat or size in P. retropinna (Fig. S.3.2).

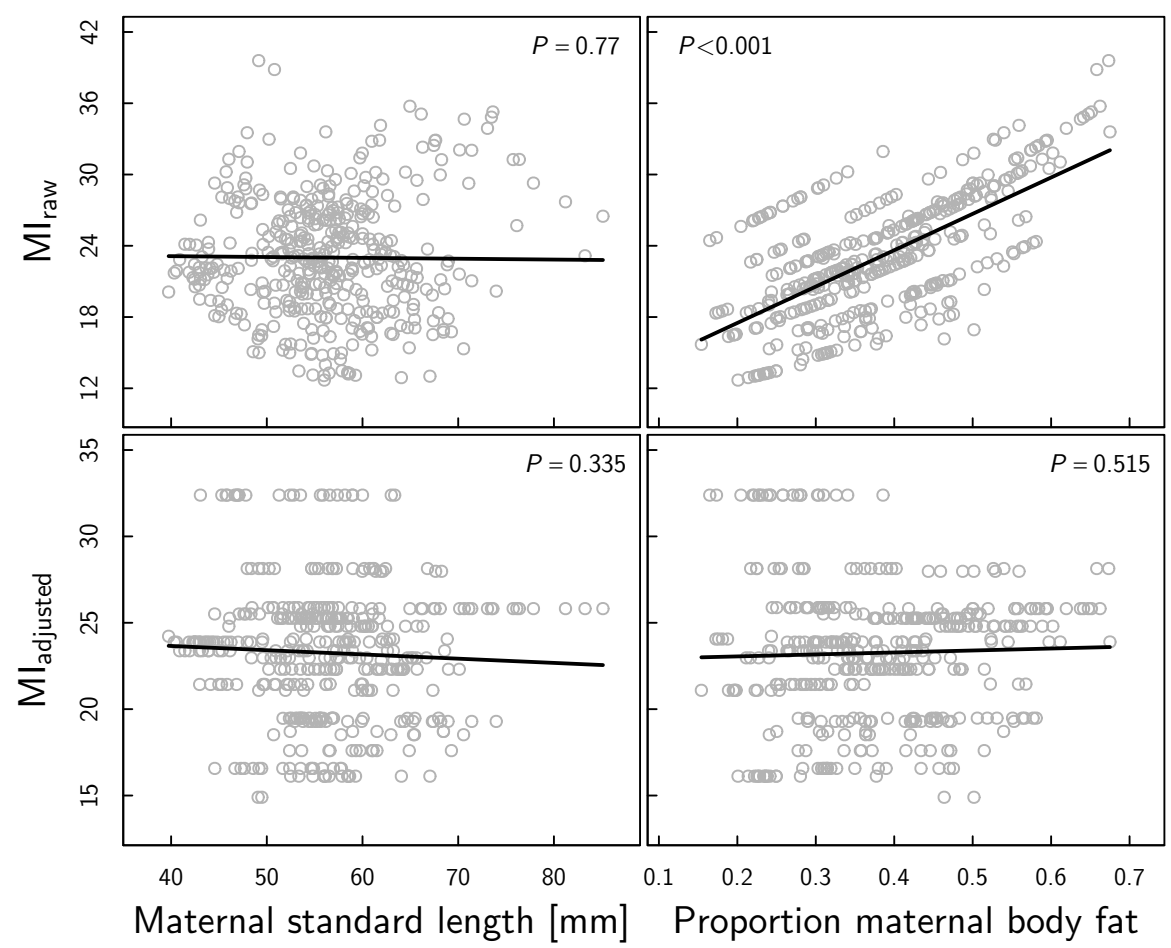

Fig. S.3.1. Association of the 'raw' Matrotrophy Indices (MI's) (upper panels) and 'adjusted' Ml's (lower panels) estimated in the model described in Equation S.3.1 with maternal standard length ( $\mathrm{mm}$ ) (left panels) and proportion of maternal body fat (right panels). The 'raw' Ml's are predicted for a given population in a specific year and for the observed values of maternal standard length and body fat, and hence, are not independent of these traits. As it was shown in Hagmayer et al. (2018), the MI is positively associated with maternal body fat, but does not correlate with maternal standard length. By contrast, the 'adjusted' Ml's are predicted for a given population in a specific year and for a female of overall average standard length and proportion of body fat. Since the 'adjusted' MI's are predicted for a female of the same length and body fat, the resulting Ml's are independent of these traits. $P$-value is given at the top. 


$\begin{array}{lll}\text { Rio Pacuar } & \nabla \text { Rio Coloradito } & \oplus \text { Rio Balsar } \\ \text { Rio Pedregoso } & \nabla \text { Rio Canaza } & \oplus \text { Rio Seco } \\ \text { Rio Ceibo } & \otimes \text { Rio Incendio } & \otimes \text { Rio Caracol } \\ \text { Rio Sucio } & * \text { Rio Lagarto } & \oplus \text { Rio Guineal }\end{array}$

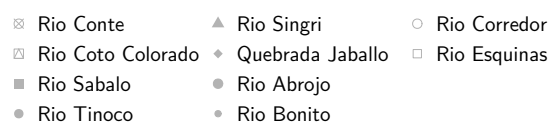

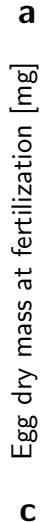

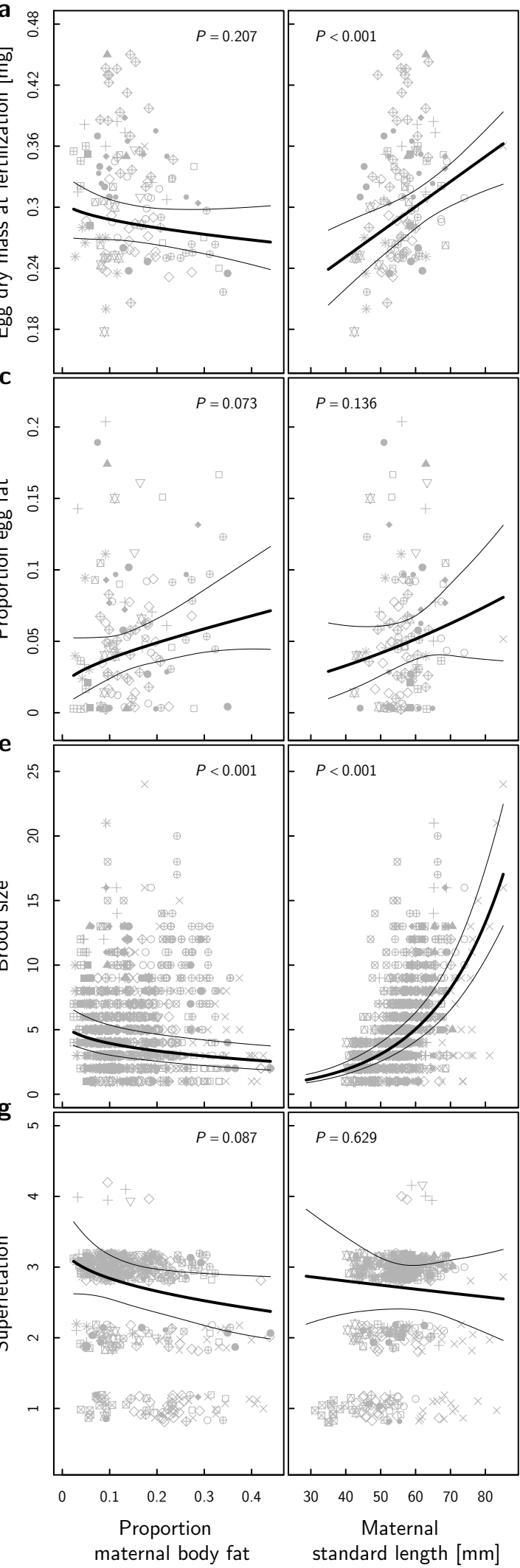

b

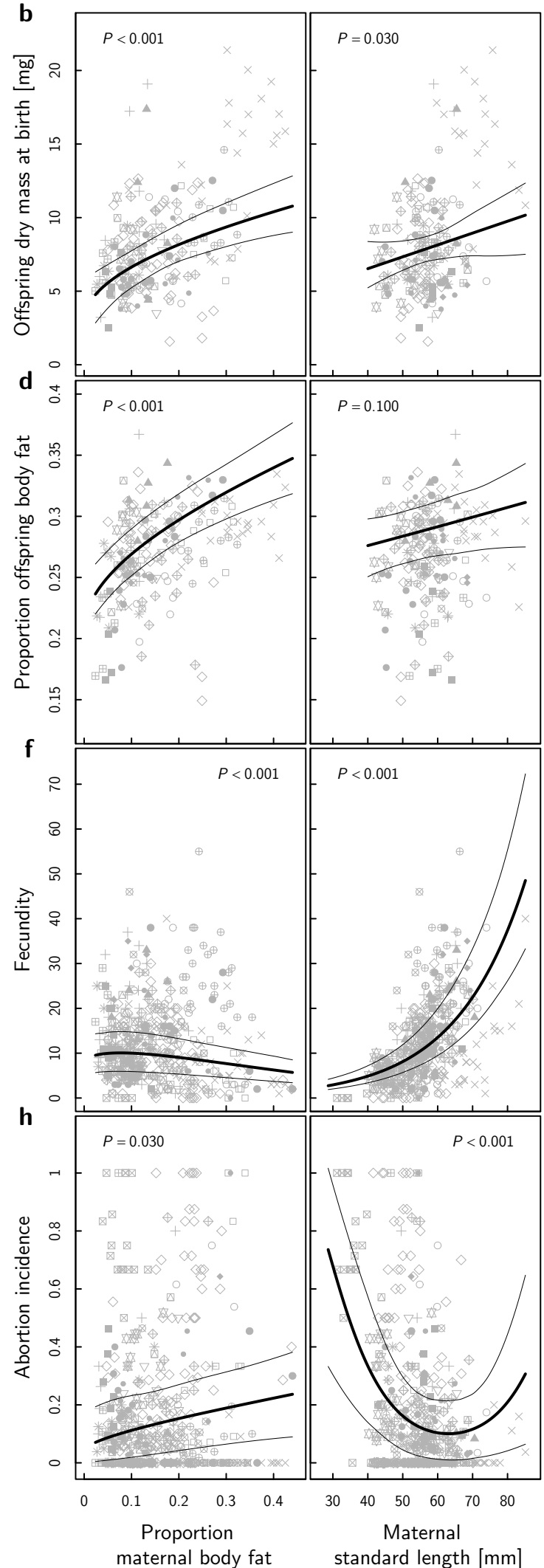


Fig. S.3.2. Life-history characteristics of Poeciliopsis retropinna in relation to maternal body fat and size. a Egg dry mass at fertilization (i.e. developmental stage 2$)(n=129)$, b offspring dry mass at birth (i.e. developmental stage 45$)(n=190)$, c proportion of egg fat at fertilization (i.e. developmental stage 2) $(n=117)$, d proportion of offspring fat at birth (i.e. developmental stage 45) $(n=190)$, e brood size $(n=943), \mathbf{f}$ maternal fecundity (i.e. number embryos) $(n=411), \mathbf{g}$ degree of superfetation $(n=449)$, and $\mathbf{h}$ abortion incidence $(n=463)( \pm 95 \% \mathrm{Cl})$ as a function of the proportion of maternal body fat (left panels) and standard length (right panels). The model predictions are based on a high predation female and account for maternal standard length (left panels) and the proportion of maternal body fat (right panels), which are kept constant at the overall population mean (i.e. $\bar{y}_{\text {body fat }}=0.16, \bar{y}_{\text {standard length }}=53 \mathrm{~mm}$ ). In $\mathbf{f}$ and $\mathbf{g}$, the developmental stage of the most-developed brood carried by the female is kept constant at the overall median (i.e. developmental stage 42.5). Data points correspond to the river-specific raw data. $P$-value is given at the top.
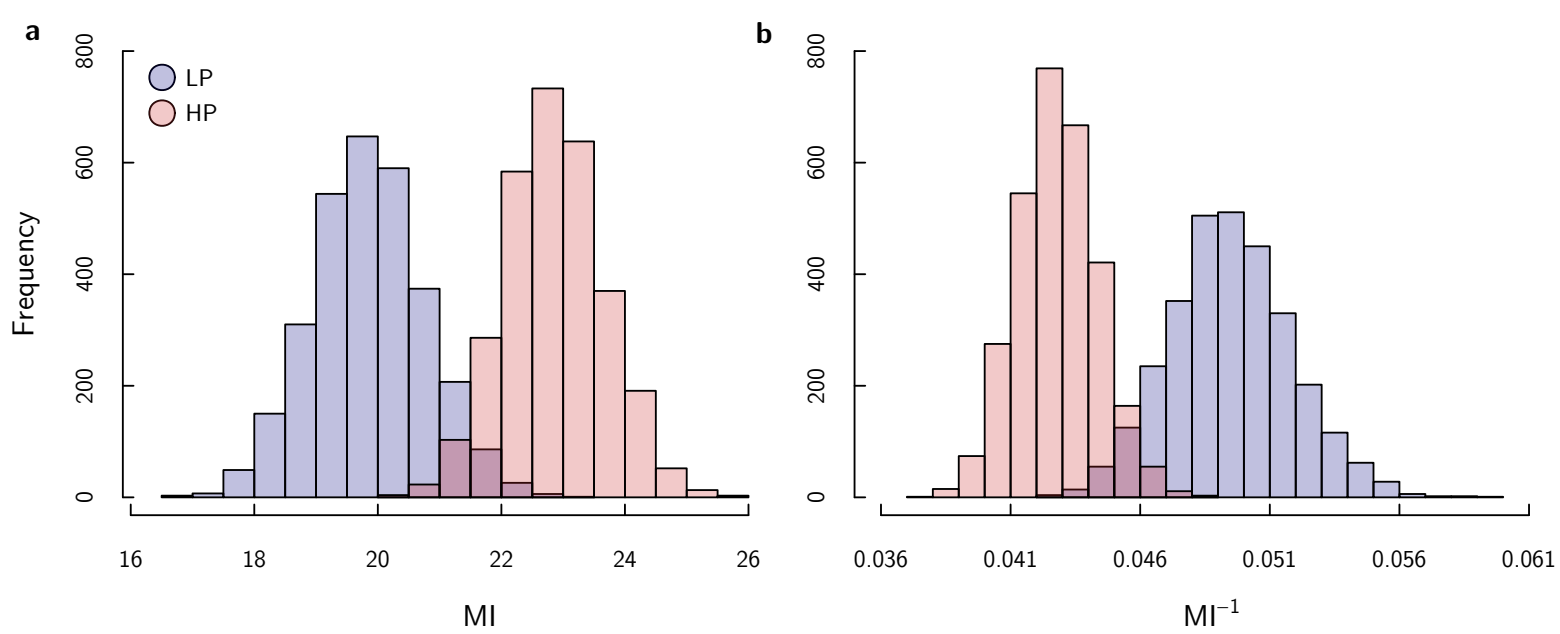

Fig. S.3.3. a Frequency distribution of the posterior estimates of the Matrotrophy Indices (Ml's) estimated in the model described in Equation S.3.2. The MI's are predicted for a given predation regime (HP: piscivorous predator species present; LP: piscivorous predator species absent) and for a female of overall average standard length (i.e. $\bar{y}_{\text {standard length }}=53 \mathrm{~mm}$ ) and proportion of body fat (i.e. $\bar{y}_{\text {body fat }}=0.16$ ). b Frequency distribution of the posterior estimates of the inverse MI's (i.e. calculated by dividing egg dry mass at fertilization by offspring mass at birth) to show that all MCMC samples are $<1$. 

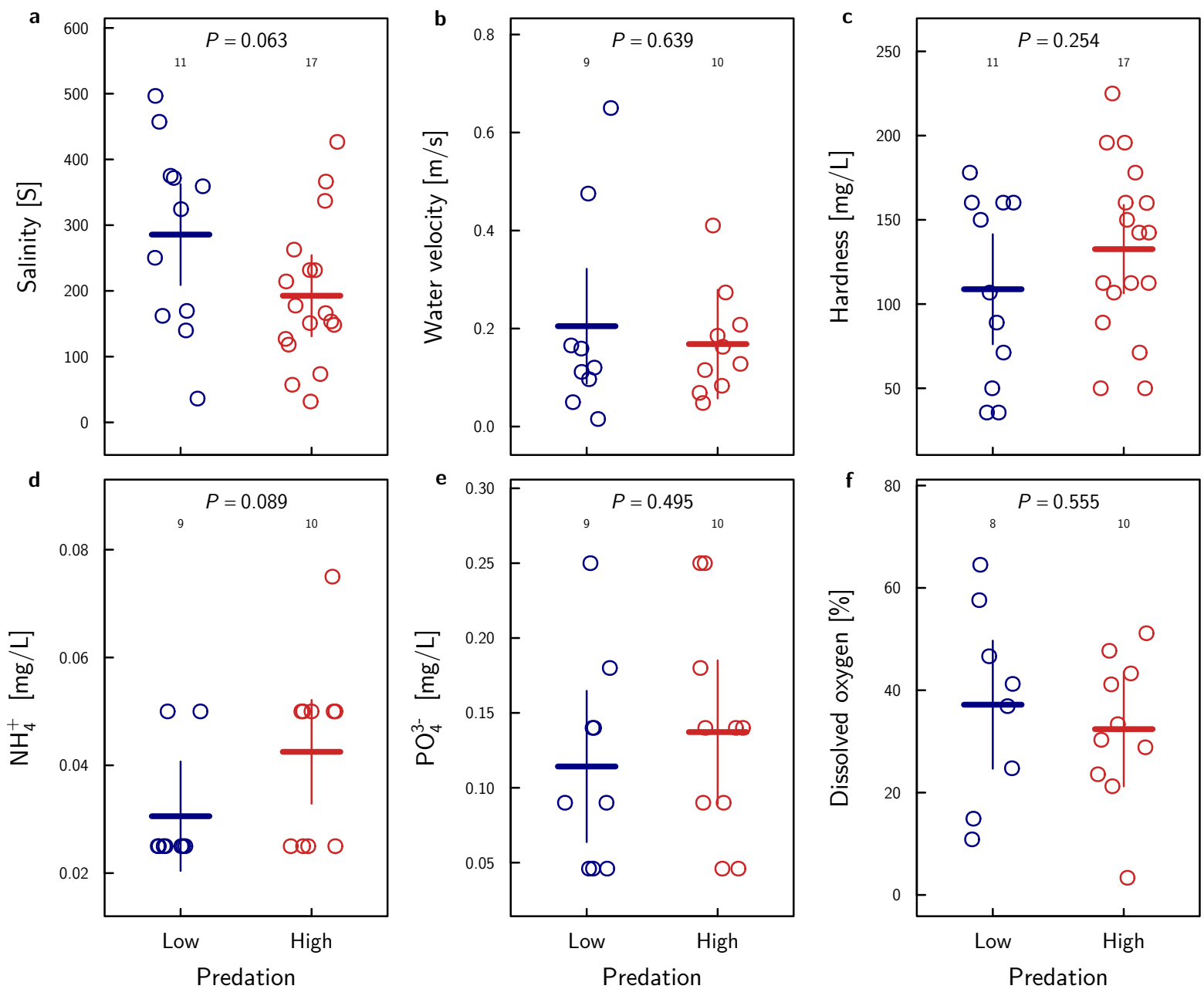

Fig. S.3.4. Water quality parameters as a function of high and low predation risk (i.e. piscivorous predator species present or absent). a Salinity, $\mathbf{b}$ mean water velocity, $\mathbf{c}$ hardness, $\mathbf{d}$ ammonium concentration $\left(\mathrm{NH}_{4}^{+}\right)$, e phosphate concentration $\left(\mathrm{PO}_{4}^{3-}\right)$, and $\mathbf{f}$ dissolved oxygen $( \pm 95 \% \mathrm{Cl})$. Data points (red: high predation; blue: low predation) correspond to the 'jittered' raw data. Sample size and $P$-value are given at the top. These data tentatively suggest that there are no obviously large differences in water quality parameters between study locations with and without predators. However, these results should be interpreted with care, because salinity and dissolved oxygen were measured only 1-3 times at each location, and hardness, ammonium concentration, and phosphate concentration were measured even only once. Water quality parameters are likely to vary during the day and throughout the year. A single or only a few measurements per location taken at different times during the day, on different days, and even in different years are therefore unlikely to accurately reflect the yearly mean local abiotic conditions experienced by the fish in each population. 


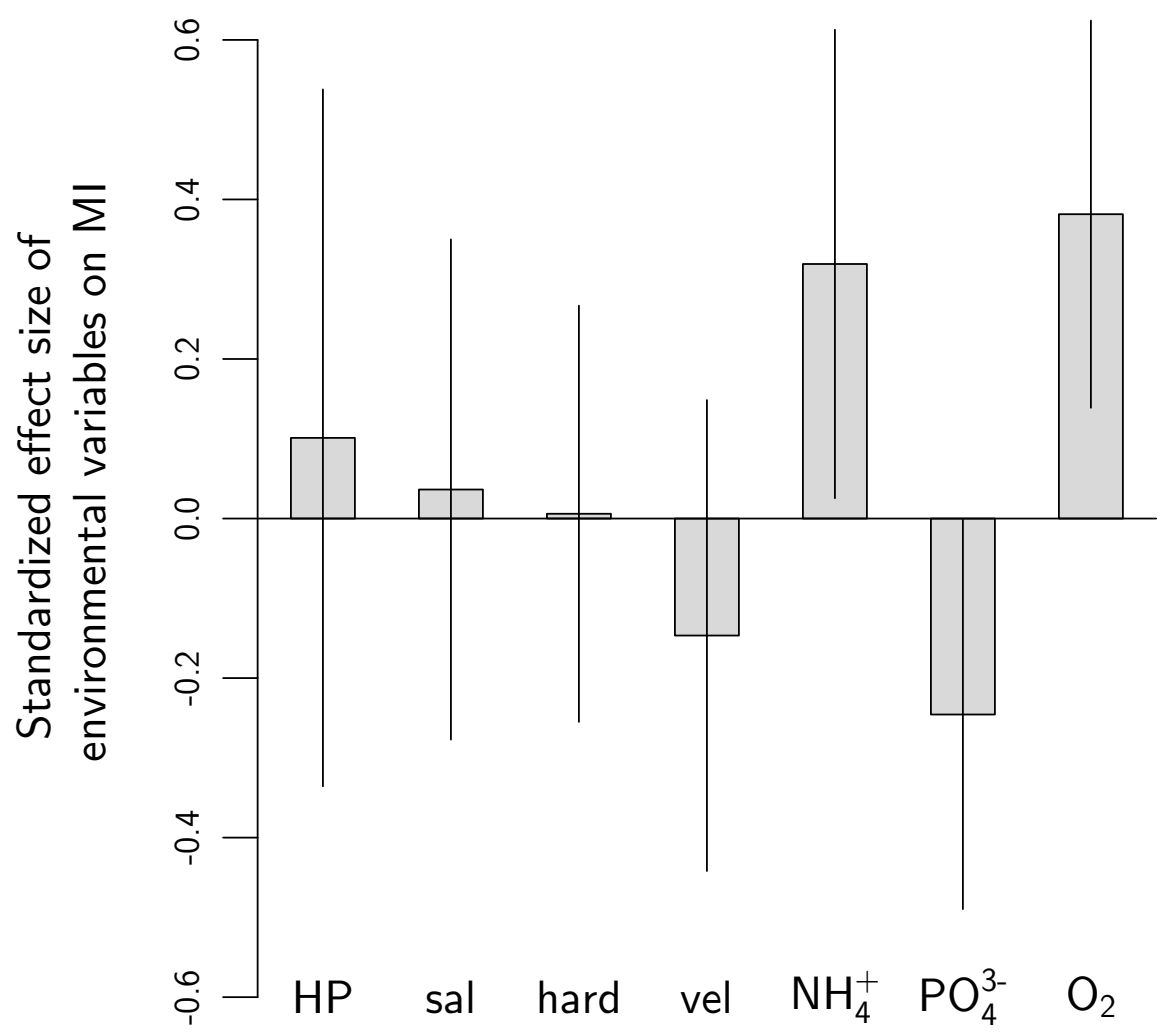

Fig. S.3.5. Standardized partial regression coefficients $\left(\beta^{*}\right)( \pm \mathrm{se})$ for the measured environmental variables on the population-specific Matrotrophy Indices (Ml's) estimated in the model described in Equation S.3.8. The population-specific Matrotrophy Indices (Ml's) were estimated in the model described in Equation S.3.1. The environmental variables include predation risk (HP: high predation, i.e. piscivorous predator species present), salinity $(\mathrm{S})$, hardness (hard), mean water velocity (vel), ammonium concentration $\left(\mathrm{NH}_{4}^{+}\right)$, phosphate concentration $\left(\mathrm{PO}_{4}^{3-}\right)$, and dissolved oxygen $\left(\mathrm{O}_{2}\right)$. The $\beta^{* \prime}$ s are equivalent to standardized effect sizes that take values between -1 and 1 (Schielzeth, 2010). Please note that salinity and dissolved oxygen were measured only 13 times at each location, and hardness, ammonium concentration, and phosphate concentration were measured even only once. The water quality parameters are likely to vary during the day and throughout the year. A single or only a few measurements per location taken at different times during the day, on different days, and even in different years are therefore unlikely to accurately reflect the yearly mean local abiotic conditions experienced by the fish in each population. Thus, care must be taken when interpreting the relationship between the $\mathrm{Ml}$ and the water quality parameters. Still, these data show that independently of the measured water quality parameters, we still predict a higher degree of placentation in high predation populations. 


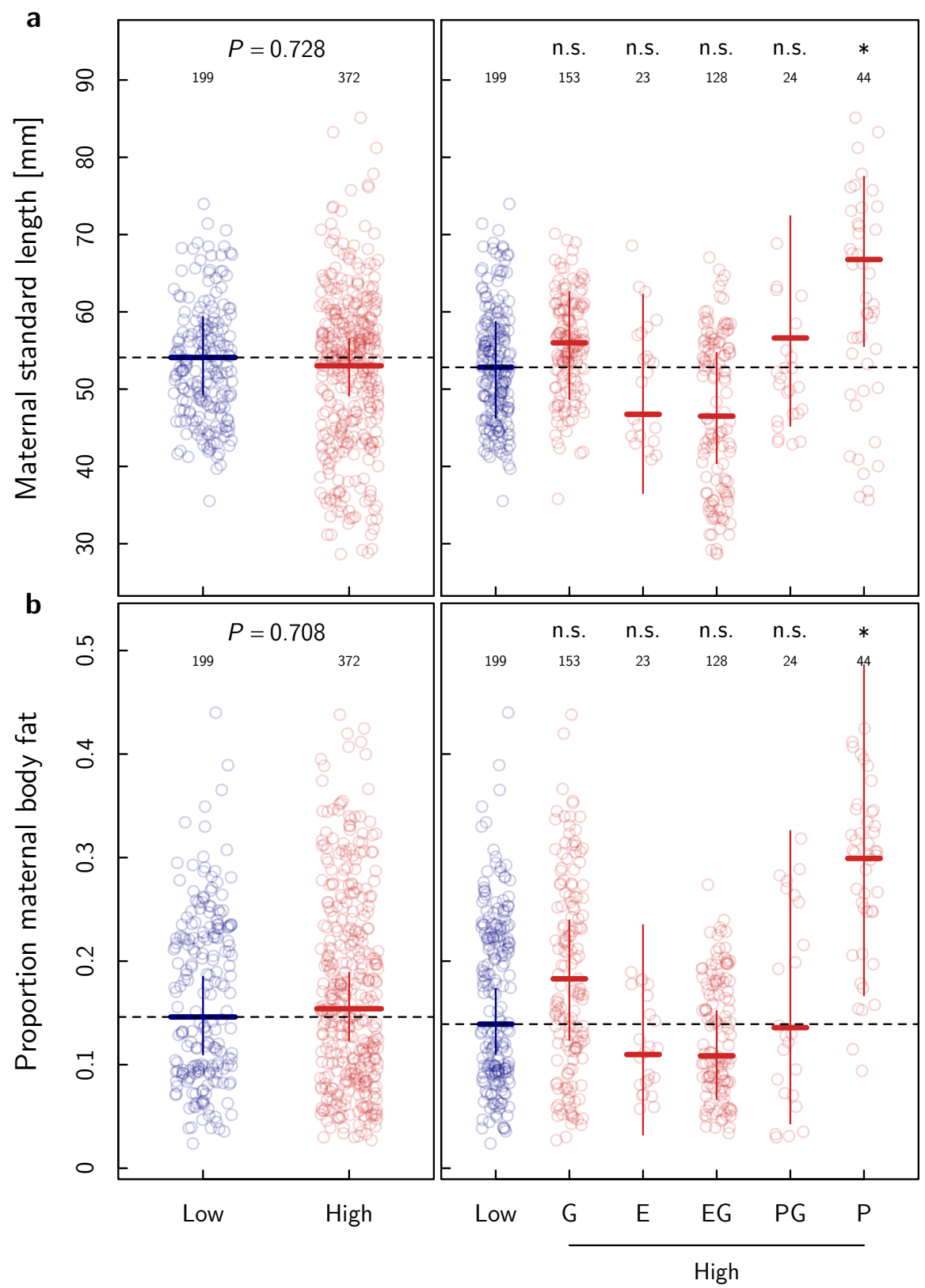

Predation

Fig. S.3.6. a Maternal standard length and b proportion of maternal body fat $( \pm 95 \% \mathrm{Cl})$ as a function of predation risk. The model predictions account for the proportion of maternal body fat in $\mathbf{a}$ and maternal standard length in $\mathbf{b}$ that are kept constant at the overall population mean (i.e. $\bar{y}_{\text {body fat }}=0.16, \bar{y}_{\text {standard length }}=53 \mathrm{~mm}$ ). left panels: high and low predation risk (i.e. piscivorous predator species present or absent); right panels: predator community. G: Gobiomorus maculatus; E: Eleotris picta; P: Parachromis dovii. Data points (red: high predation; blue: low predation) correspond to the 'jittered' raw data. Sample size and $P$-value are given at the top. Significant codes: $P<0.001^{* * *},<0.01^{* *}, \leq 0.05^{*},>0.05$ n.s. 

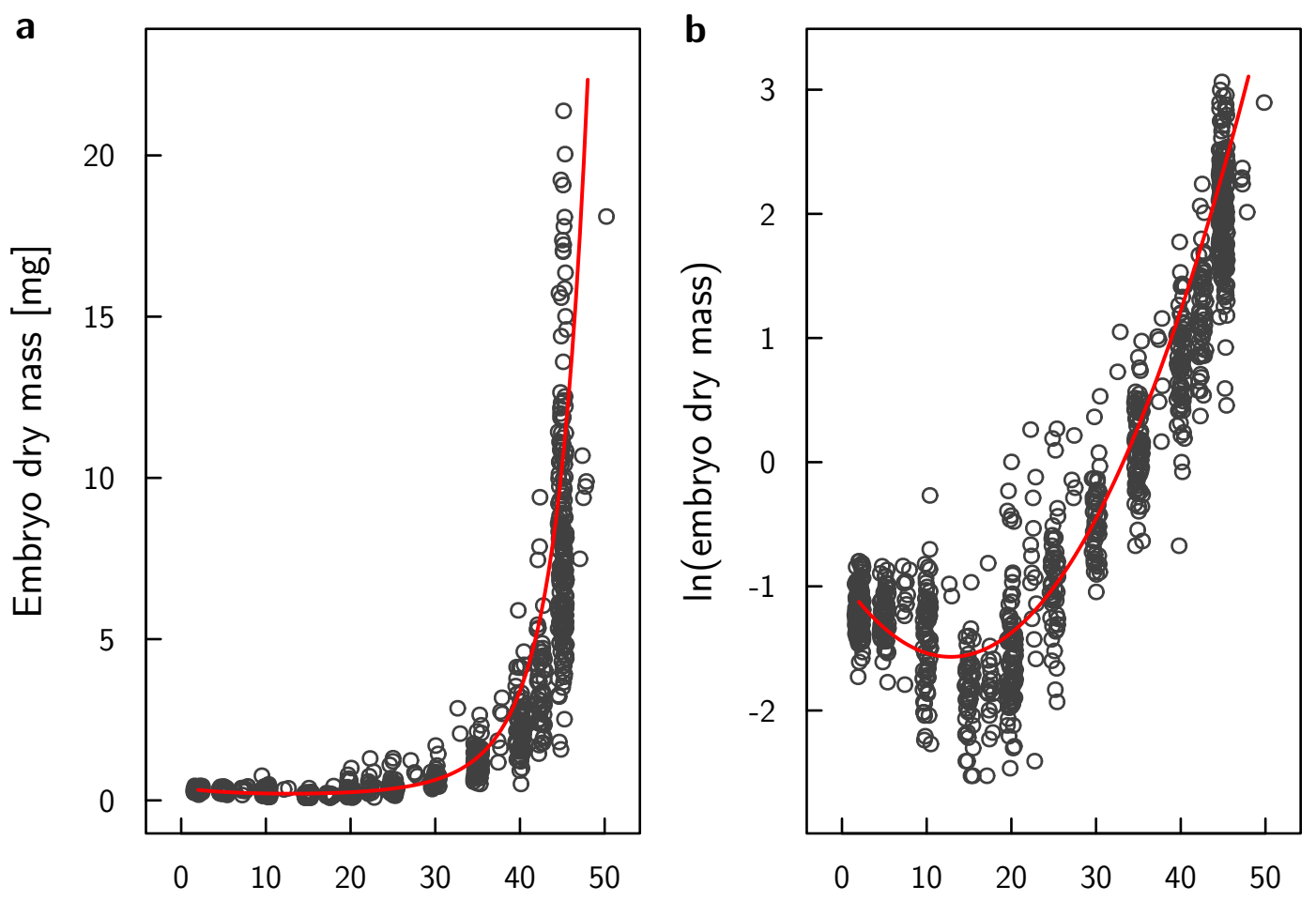

Developmental stage

Fig. S.3.7. a Embryo dry mass [mg] and $\mathbf{b}$ In-transformed embryo dry mass as a function of the developmental stage of embryos (2-50) estimated in the model described in Equation S.3.9. The model prediction (red) accounts for maternal dry mass that is kept constant at the overall population mean (i.e. $\bar{y}_{\text {dry mass }}=0.982 \mathrm{~g}$ ). Data points (dark grey) correspond to the 'jittered' raw data. Please note that embryo dry mass decreases until developmental stage 13 . The placenta might not be fully functional during early embryo development. Thus, the mass loss as a result of metabolic processes may outweigh the mass gain as a result of placental provisioning early in pregnancy. 
Predation drives placental evolution
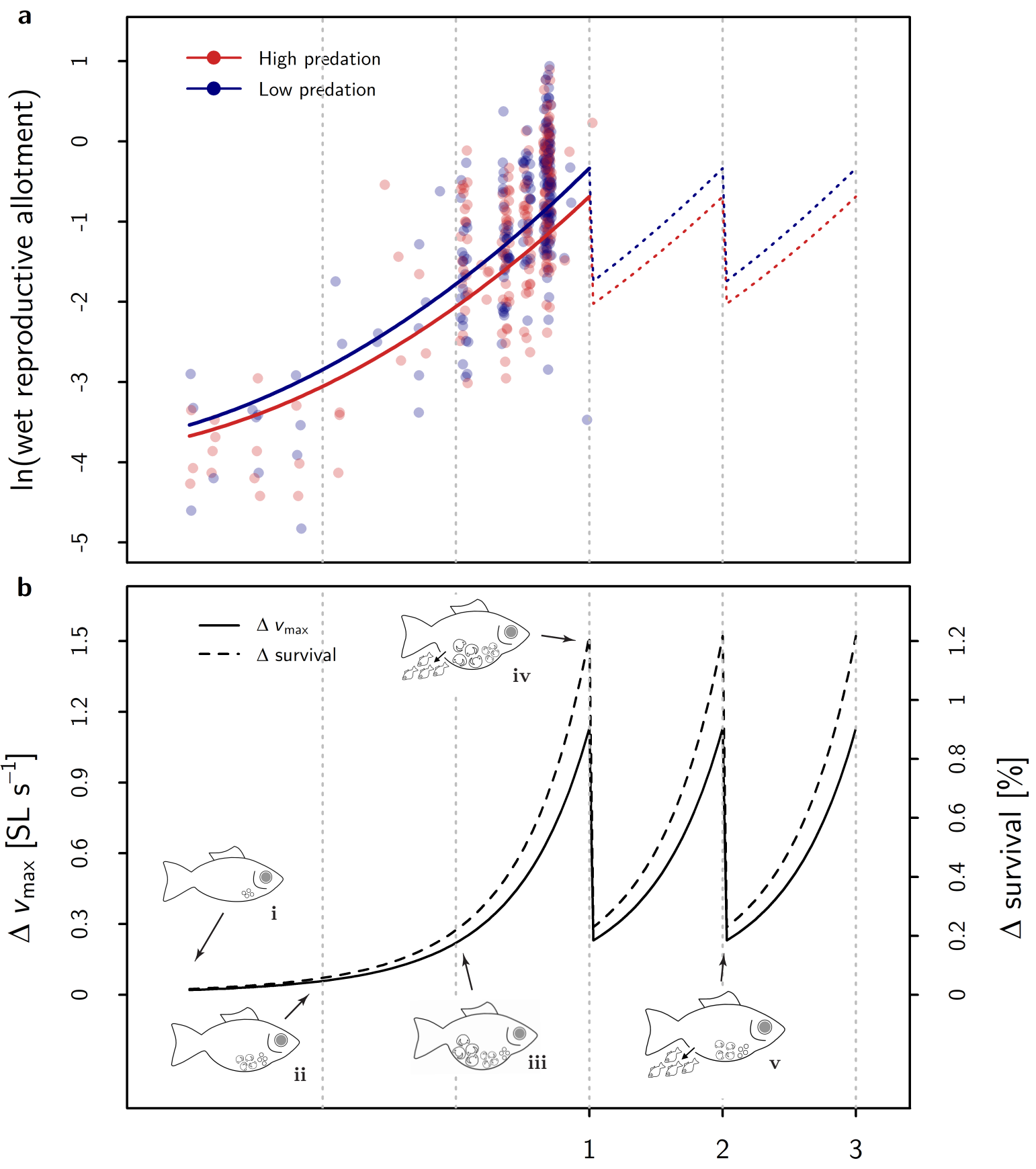

Number of produced broods

Time 
Fig. S.3.8. a In-transformed absolute wet reproductive allotment as a function of time and high (red) and low (blue) predation risk (i.e. piscivorous predator species present or absent) in $P$. retropinna. Data points correspond to the 'jittered' raw data. The developmental stage of the most developed brood was used to define the time from fertilization to parturition of the first brood (thick solid line). Continuous brood production (i.e. superfetation) subsequently leads to a repetitive pattern of frequent parturition (thin dotted line). Note that high predation females produce smaller eggs at fertilization, which contributes to the decreased wet reproductive allotment at fertilization. Despite producing the same size and number of offspring, superfetation prevents the difference in reproductive allotment at fertilization from becoming zero towards the end of gestation. The difference in reproductive allotment between high and low predation populations is largest before the parturition of a brood (dotted grey lines). b Predicted difference in maximum escape velocity $\left(\mathrm{SL} \cdot \mathrm{s}^{-1}\right.$; solid line) and survival probability (dotted line) between high and low predation females based on Poeciliopsis turneri (Fleuren et al., 2019), and Poecilia reticulata (Walker et al., 2005), respectively. i Female early in pregnancy carrying a single brood at developmental stage 2 (i.e. eggs at fertilization); ii female fertilizes a second brood; most-developed brood at developmental stage 18; iii female fertilizes a third brood; intermediate-developed brood at developmental stage 18; most-developed brood at developmental stage 34; iv least-developed brood at developmental stage 18; intermediate-developed brood at developmental stage 34; most-developed brood ready to get born (i.e. stage 50); $\mathbf{v}$ female fertilizes a third brood; intermediate-developed brood at developmental stage 18; most-developed brood at developmental stage 34 .

Fig. S.3.9. Simulated frequency of a high (HP) and low (LP) predation genotype over time (i.e. generations). The HP genotype is assumed to have a survival advantage of $1.2 \%$, and there is no mixing of the genotypes. The initial frequency $\left(p_{0}^{2}\right)$ of the advantageous genotype in the population is assumed to be 0.01 . The dotted line corresponds to the point in time, at which the advantageous genotype makes up $95 \%$ of the population.

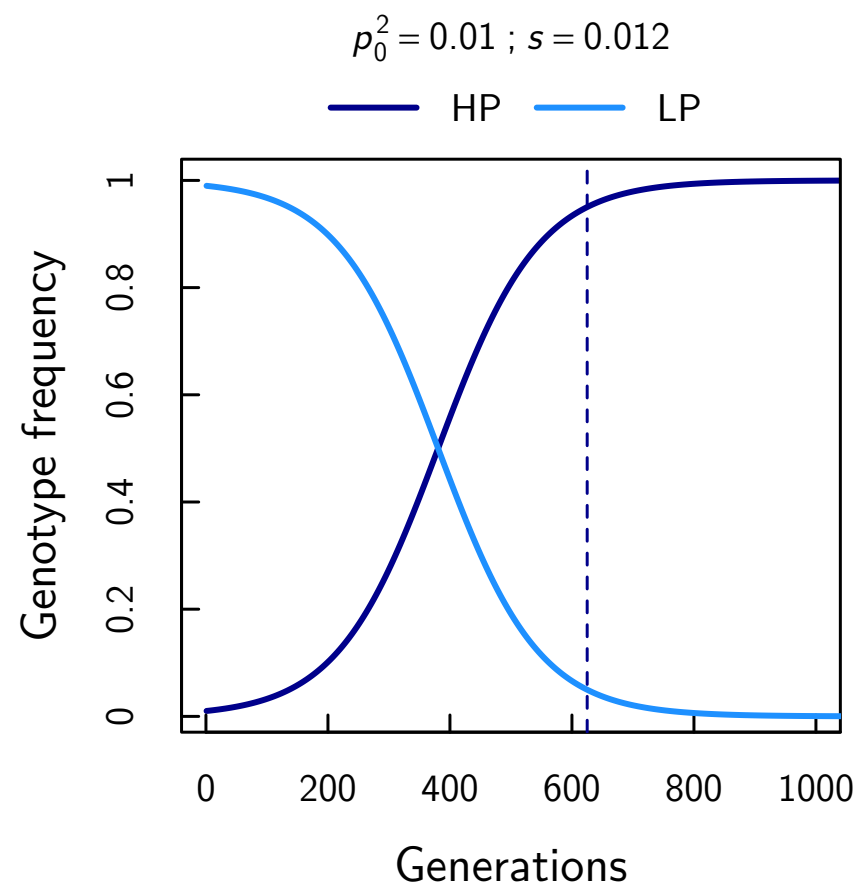


Table S.3.1. Sampling locations (plus coordinates), sampling dates, predation risk (HP: piscivorous predator species present; LP: piscivorous predator species absent), number of collected $(n)$ and pregnant ( $\left.n_{\text {preg }}\right)$ females at each location, and population-specific Matrotrophy Indices (MI's). The Ml's are estimated using the Bayesian programming environment JAGS (Plummer, 2003) and are predicted for a given population in a specific year and for a female of overall average standard length and proportion of body fat. The $\mathrm{MI}_{\text {raw }}$ 's are predicted for the observed, rather than the average maternal body fat and standard length.

\begin{tabular}{|c|c|c|c|c|c|c|c|c|}
\hline Location & River & Predation & Coordinates & Sampling date & $n$ & $n_{\text {preg }}$ & $\mathrm{MI}$ & $\mathrm{MI}_{\text {raw }}$ \\
\hline Canaza & Rio Canaza & $\mathrm{HP}$ & $\mathrm{N} 08^{\circ} 39^{\prime}, \mathrm{W} 83^{\circ} 11^{\prime}$ & 29.03 .2013 & 15 & 2 & 22.80 & 22.70 \\
\hline Ceibo downstream & Rio Ceibo & $\mathrm{HP}$ & $\mathrm{N} 09^{\circ} 09^{\prime}, \mathrm{W} 83^{\circ} 22^{\prime}$ & 26.03 .2013 & 24 & 8 & 24.02 & 21.52 \\
\hline Coloradito downstream & Rio Coloradito & HP & N $08^{\circ} 36^{\prime}$, W $82^{\circ} 54^{\prime}$ & 28.03.2013 & 26 & 24 & 28.10 & 27.42 \\
\hline Incendio & Rio Incendio & $\mathrm{HP}$ & $\mathrm{N} 08^{\circ} 27^{\prime}, \mathrm{W} 82^{\circ} 60^{\prime}$ & 29.03.2013 & 27 & 17 & 24.17 & 20.07 \\
\hline Lagarto & Rio Lagarto & $\mathrm{HP}$ & $\mathrm{N} 08^{\circ} 41^{\prime} \mathrm{W} 83^{\circ} 05^{\prime}$ & 30.03 .2013 & 25 & 23 & 32.34 & 27.43 \\
\hline Pacuar & Rio Pacuar & $\mathrm{HP}$ & $\mathrm{N} 09^{\circ} 21^{\prime}, \mathrm{W} 83^{\circ} 44^{\prime}$ & 25.03 .2013 & 20 & 3 & 21.41 & 25.82 \\
\hline Pedregoso & Rio Pedregoso & LP & $\mathrm{N} 09^{\circ} 21^{\prime}, \mathrm{W} 83^{\circ} 43^{\prime}$ & 25.03.2013 & 30 & 0 & NA & NA \\
\hline Sucio & Rio Sucio & $\mathrm{HP}$ & $\mathrm{N} 08^{\circ} 49^{\prime}, \mathrm{W} 82^{\circ} 55^{\prime}$ & 27.03 .2013 & 24 & 23 & 25.78 & 31.36 \\
\hline Copal & Rio Sucio & LP & $\mathrm{N} 08^{\circ} 48^{\prime}, \mathrm{W} 82^{\circ} 55^{\prime}$ & 27.03.2013 & 10 & 6 & 27.95 & 31.61 \\
\hline Coloradito middle & Rio Coloradito & LP & $\mathrm{N} 08^{\circ} 40^{\prime}, \mathrm{W} 82^{\circ} 53^{\prime}$ & 28.03.2013 & 28 & 23 & 14.87 & 16.52 \\
\hline Canaza & Rio Canaza & HP & $\mathrm{N} 08^{\circ} 38^{\prime}, \mathrm{W} 83^{\circ} 10^{\prime}$ & 08.03 .2017 & 10 & 10 & 19.43 & 19.10 \\
\hline Caracol & Rio Caracol & $\mathrm{HP}$ & $\mathrm{N} 08^{\circ} 39^{\prime}, \mathrm{W} 83^{\circ} 00^{\prime}$ & 09.03 .2017 & 25 & 25 & 23.83 & 21.73 \\
\hline Claro & Rio Coto Colorado & $\mathrm{HP}$ & $\mathrm{N} 08^{\circ} 41^{\prime}, \mathrm{W} 83^{\circ} 06^{\prime}$ & 09.03 .2017 & 23 & 23 & 23.33 & 21.75 \\
\hline Conte & Rio Conte & $\mathrm{HP}$ & $\mathrm{N} 08^{\circ} 26^{\prime}, \mathrm{W} 83^{\circ} 02^{\prime}$ & 07.03 .2017 & 10 & 10 & 25.45 & 26.52 \\
\hline Palmar Norte & Rio Balsar & $\mathrm{HP}$ & $\mathrm{N} 08^{\circ} 59^{\prime}, \mathrm{W} 83^{\circ} 31^{\prime}$ & 11.03 .2017 & 32 & 31 & 22.26 & 22.43 \\
\hline Seco & Rio Seco & $\mathrm{HP}$ & N $08^{\circ} 39^{\prime}$, W $82^{\circ} 56^{\prime}$ & 06.03.2017 & 25 & 23 & 24.75 & 28.79 \\
\hline Coloradito middle & Rio Coloradito & $\mathrm{HP}$ & $\mathrm{N} 08^{\circ} 35^{\prime}, \mathrm{W} 82^{\circ} 52^{\prime}$ & 05.03 .2017 & 37 & 36 & 25.21 & 26.22 \\
\hline Porto Grande & Rio Guineal & LP & $\mathrm{N} 09^{\circ} 01^{\prime}, \mathrm{W} 83^{\circ} 09^{\prime}$ & 01.03 .2017 & 11 & 11 & 21.05 & 16.71 \\
\hline Ceibo upstream & Rio Ceibo & LP & $\mathrm{N} 09^{\circ} 12^{\prime}, \mathrm{W} 83^{\circ} 18^{\prime}$ & 27.02 .2017 & 22 & 21 & 22.92 & 21.45 \\
\hline Bonito & Rio Bonito & $\mathrm{HP}$ & $\mathrm{N} 08^{\circ} 43^{\prime}, \mathrm{W} 83^{\circ} 12^{\prime}$ & 25.03 .2018 & 15 & 14 & 17.57 & 17.59 \\
\hline Esquinas & Rio Esquinas & $\mathrm{HP}$ & $\mathrm{N} 08^{\circ} 44^{\prime}, \mathrm{W} 83^{\circ} 10^{\prime}$ & 24.03.2018 & 20 & 17 & 19.44 & 22.41 \\
\hline Sabalo & Rio Sabalo & $\mathrm{HP}$ & $\mathrm{N} 08^{\circ} 52^{\prime}, \mathrm{W} 83^{\circ} 19^{\prime}$ & 26.03 .2018 & 15 & 15 & 16.09 & 13.37 \\
\hline Abrojo & Rio Abrojo & LP & N $08^{\circ} 37^{\prime}$, W $82^{\circ} 52^{\prime}$ & 23.03.2018 & 12 & 12 & 23.84 & 26.61 \\
\hline Corredor & Rio Corredor & LP & $\mathrm{N} 08^{\circ} 40^{\prime}, \mathrm{W} 82^{\circ} 54^{\prime}$ & 23.03 .2018 & 20 & 20 & 19.25 & 19.43 \\
\hline Jaballo & Quebrada Jaballo & LP & $\mathrm{N} 08^{\circ} 57^{\prime}, \mathrm{W} 83^{\circ} 06^{\prime}$ & 16.03 .2018 & 5 & 5 & 18.68 & 17.88 \\
\hline Pavon & Rio Balsar & LP & $\mathrm{N} 09^{\circ} 00^{\prime}, \mathrm{W} 83^{\circ} 31^{\prime}$ & 26.03 .2018 & 17 & 17 & 16.54 & 15.79 \\
\hline Singri & Rio Singri & LP & $\mathrm{N} 08^{\circ} 59^{\prime}, \mathrm{W} 83^{\circ} 06^{\prime}$ & 15.03 .2018 & 6 & 6 & 18.48 & 17.28 \\
\hline Tinoco & Rio Tinoco & LP & $\mathrm{N} 08^{\circ} 54^{\prime}, \mathrm{W} 83^{\circ} 22^{\prime}$ & 26.03.2018 & 18 & 18 & 21.39 & 18.97 \\
\hline Coloradito upstream & Rio Coloradito & LP & $\mathrm{N} 08^{\circ} 35^{\prime}, \mathrm{W} 82^{\circ} 51^{\prime}$ & 25.03 .2018 & 20 & 20 & 25.83 & 22.83 \\
\hline Total & & & & & 572 & 463 & & \\
\hline
\end{tabular}





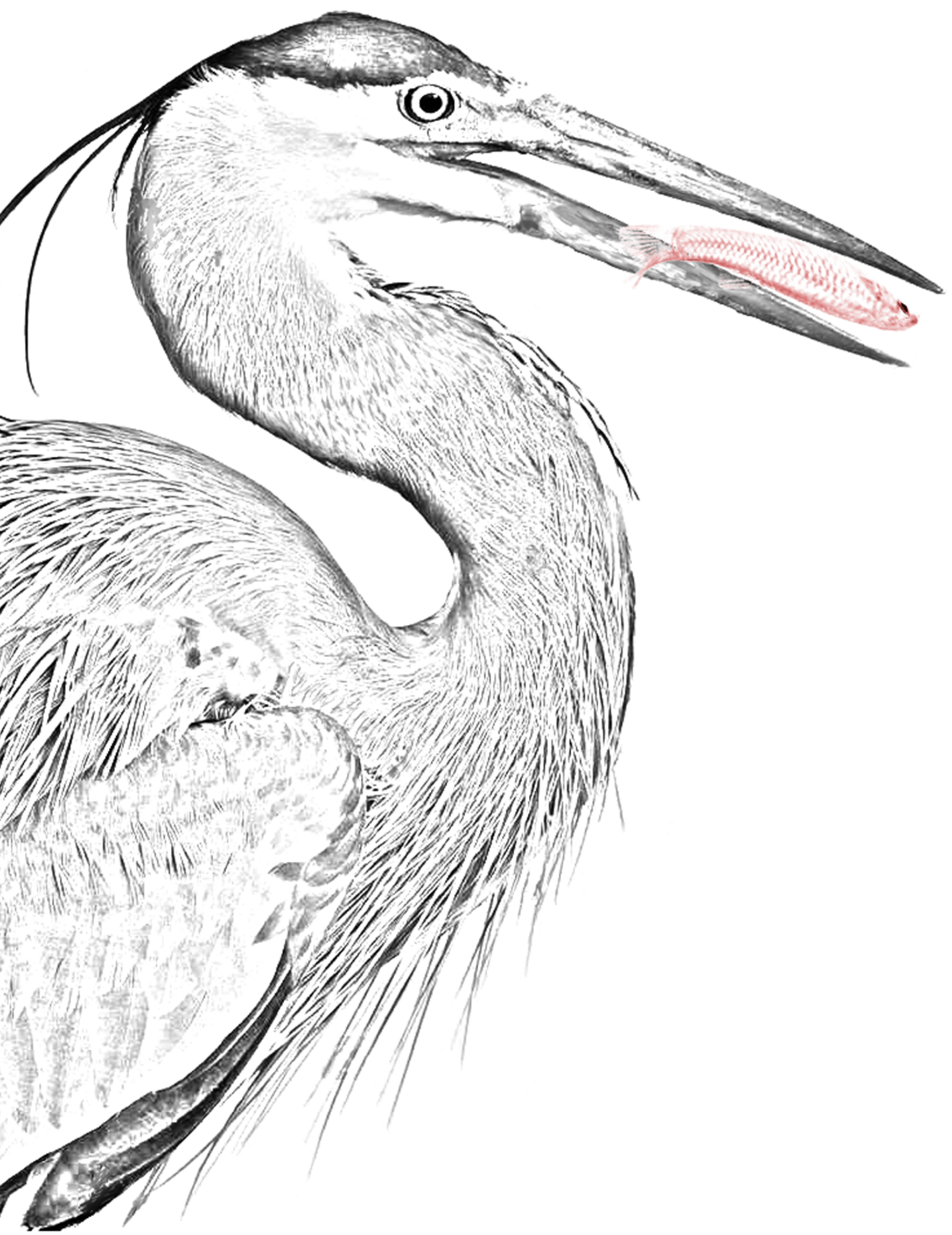




\section{Differences in ontogenetic and diurnal mi- crohabitat selection by sympatric live-bearing fish species with different reproductive modes}

Andres Hagmayer ${ }^{1}$, Andrew I. Furness ${ }^{2,3}$ \& Bart J.A. Pollux ${ }^{1}$

1 Department of Animal Sciences, Wageningen University, 6708 WD Wageningen, The Netherlands

2 Department of Ecology and Evolutionary Biology, University of California, CA 92697 Irvine, USA

${ }^{3}$ Department of Biological and Marine Sciences, University of Hull, HU6 7RX Hull, UK 


\section{Abstract}

A pregnancy imposes a heavy reproductive burden on females. Some live-bearing species have evolved reproductive adaptations to reduce this burden, which may influence their ability to utilize specific microhabitats. We investigate whether two such reproductive adaptations, placentation (embryo provisioning via a placenta) and superfetation (the ability to carry multiple broods at various developmental stages), influence microhabitat selection by five sympatric Costa Rican live-bearing fish species (family Poeciliidae). Theory predicts that placentation and superfetation should both reduce the reproductive burden of females during pregnancy, improve their body streamlining, and swimming performance, and consequently allow them to utilize 'more performance-demanding' microhabitats. Here we apply underwater visual fish surveys to test a key prediction of this hypothesis, which is that the presence of these two reproductive adaptations is correlated with the utilization of microhabitats in the river that are characterized by a higher-flow velocity. Consistent with our predictions, we observed significant interspecific differences in daytime microhabitat use: species that had both placentation and superfetation were found in deeper and faster-flowing parts of the river, species that lacked both adaptations were confined to shallow slow-flowing areas, and species with one adaptation (i.e. only superfetation) inhabited intermediate areas. This interspecific daytime microhabitat use was strongest in reproductive adults, intermediate in immatures, and absent in juveniles (the latter of which were all found in shallow low-velocity zones), suggesting that ontogeny influences species-specific microhabitat use. Finally, at night, all fishes, regardless of the species or age-class, congregated in shallow slow-flowing waters to rest (sleep) on the river bottom. Taken together, our results suggest that placentation and superfetation may be hitherto unrecognized reproductive features that help to understand differences in ontogenetic and diurnal microhabitat preferences between sympatric live-bearing fish species living in environments characterized by large flow variation.

Keywords: habitat selection, matrotrophy, placenta, Poeciliidae, superfetation

\subsection{Introduction}

Microhabitat selection affects community assembly and structure in response to a variety of biotic and abiotic gradients (e.g. competition, predation risk, parasites, resource availability) (Rosenzweig, 1991; Morris, 2003). It enables the use of a different set of local resources by different species, minimizing competition with co-occurring species (Bolnick et al., 2003, 2007). Microhabitat selection results from optimization processes involving individual fitness costs and benefits (Sibly \& McFarland, 1976). For instance, in environments where patches vary in food availability, optimal foraging theory predicts that individuals should prefer habitats with high food density (MacArthur \& Pianka, 1966). However, when these patches are simultaneously subject to high predation risk, individu- 
als must balance the conflicting demands of food acquisition and predator avoidance (Sih, 1980). Different species may balance these conflicting demands (feeding versus predator avoidance) in different ways, resulting in species-specific differences in microhabitat selection and affecting the spatial and temporal dynamics of species interactions (Morris, 2003). Microhabitat selection is therefore an important mechanism that allows for the competitive coexistence of species (Rosenzweig, 1981).

Microhabitat selection not only differs among species but can also vary within species. For instance, microhabitat use can change throughout an individual's life depending on their age, size or developmental stage (i.e. ontogenetic microhabitat shifts). Size-dependent predation risk was shown to account for differences in habitat use between bluegill sunfish size classes (Lepomis macrochirus), with large bluegills preferring open-water habitats with high foraging returns and small bluegills choosing less profitable habitats close to vegetation to avoid predation (Mittelbach, 1981; Werner \& Hall, 1988). Likewise, armored catfish (Loricariidae) display size-specific spatial distributions, with small catfish preferring shallow water to reduce predation risk by piscivorous predators while large catfish avoid shallow water where they are vulnerable to avian predators (Power, 1984). In coastal lagoons, many fish species display size-related shifts from settlement habitats (larvae) to nursery areas (juveniles) and subsequently to nearby coral reefs (adults). Here ontogenetic shifts in (micro)habitat use may be related to ontogenetic changes in dietary preferences or susceptibility to predation (Werner, 1984; Cocheret de la Morinière et al., 2002, 2003a,b; Pollux et al., 2007).

Many species furthermore display pronounced day-night shifts in habitat use (diurnal microhabitat shifts). Well-known examples include the diurnal vertical migration of freshwater and marine pelagic invertebrates from deeper water during the day to avoid visually orienting predators, to shallower surface waters at night to feed (Bollens \& Frost, 1989; Bollens et al., 1992; Kaartvedt et al., 2007; Sainmont et al., 2013). Juvenile Atlantic salmon (Salmo salar L.) furthermore show a temperature-dependent shift in diel activity and habitat use, which is likely the result of maximizing feeding efficiency in summer, and reducing predation risk in winter (Fraser et al., 1993). The Eurasian lynx (Lynx lynx) selects open habitats at night where prey is more abundant and shifts to dense understory cover and rugged terrain during the day to avoid human activity (Filla et al., 2017). Such diel differences in activity and microhabitat use are often the result of a complex trade-off between feeding and avoiding predators (Metcalfe et al., 1999; Fraser et al., 2004; van der Vinne et al., 2019).

Finally, microhabitat selection can also change in individuals depending on their reproductive state (reproductive microhabitat shifts). Gravid and pregnant females, for example, may temporarily select 'low performance-demanding' microhabitats: e.g. areas where they are less prone to predation or, in stream ecosystems, where they are less exposed to strong currents. The reason is that gravidity and pregnancy may negatively affect the locomotor performance of females (Seigel et al., 1987; Plaut, 2002; Noren et al., 2011; Fleuren et al., 2019), increasing their susceptibility to predation (Pollux et al., 2009; Pires et al., 
2011). This risk is compounded by the fact that predators often prefer gravid or pregnant females (Trexler et al., 1994), because they are large and (due to the presence of eggs or embryos) represent a high-quality resource that is rich in energy and nutrition. Pregnant Bighorn Sheep (Ovis canadensis), for instance, spend less time in optimal feeding areas where the mortality risk is highest, compared to females that recently gave birth (Berger, 1991). Moreover, gravid three-spined stickle-back (Gasterosteus aculeatus) remain closer to refuges when inhabiting lakes with predators than their non-gravid counterparts (Rodewald \& Foster, 1998). Similarly, the Trinidadian guppy (Poecilia reticulata) uses habitats with lower water velocity late in pregnancy to offset some of the performance-related costs of pregnancy (Banet et al., 2016). Thus, when gravidity or pregnancy are associated with increased vulnerability to high performance-demanding conditions, this is likely to induce a shift in habitat use to less performance-demanding microhabitats.

Here, we study microhabitat selection (Allee et al., 1949) by five different live-bearing fish species belonging to the family Poeciliidae (Poeciliopsis retropinna, P. paucimaculata, P. turrubarensis, Poecilia gillii, and Brachyrhaphis roseni) that co-occur in rivers and streams in Costa Rica. We quantify differences in diurnal, ontogenetic, and reproductive microhabitat use among these species and their piscivorous predator (Gobiomorus maculatus). These five co-occurring poeciliid fish species are of similar size and generally prefer similar environmental conditions (Bussing, 2002; Meyer, 2015). However, they differ in the absence/presence of two reproductive adaptations (Table 4.1). The first reproductive adaptation is the placenta: $P$. turrubarensis, $P$. gillii, and $B$. roseni are lecithotrophic (or 'yolk-feeding'), committing all nutrients required for development during pregnancy to the eggs prior to fertilization, while P. retropinna and P. paucimaculata are placentotrophic (i.e. 'mother-feeding') provisioning nutrients to the developing embryos throughout pregnancy via a placenta (Pollux et al., 2014; Furness et al., 2019). The second reproductive adaptation is superfetation: $P$ retropinna, $P$. paucimaculata, and $P$. turrubarensis have superfetation, which means that they are able to carry multiple broods at different developmental stages, while P. gillii and B. roseni lack superfetation (Pollux et al., 2009).

Theory predicts that (different combinations of) these two reproductive adaptations may be associated with differences in microhabitat use. The reason is that the presence of the placenta and superfetation are both thought to reduce a female's reproductive burden during pregnancy (Reznick et al., 2007; Pollux et al., 2009; Bassar et al., 2014; Hagmayer et al., 2020c). A reduced reproductive burden has further been associated with an enhanced body streamlining and improved locomotor performance of females during pregnancy (Thibault \& Schultz, 1978; Pollux et al., 2009; Zúñiga-Vega et al., 2010; Pires et al., 2011; Fleuren et al., 2018, 2019; Quicazan-Rubio et al., 2019), potentially allowing the utilization of different stream (micro)habitats (Banet et al., 2016). Here, we test a key prediction of this hypothesis, namely that the two reproductive adaptations should be associated with a species' microhabitat utilization. Specifically, (i) we predict that reproductive adults of species with a placenta and/or superfetation will inhabit relatively 
deeper, faster-flowing sections in the middle of the river compared to species that lack both reproductive adaptations. (ii) Furthermore, we know from preliminary nocturnal observations (Hagmayer, Furness \& Pollux, personal observations) that poeciliid species become inactive at night and tend to move to the shallows to rest. We therefore predict that if adults of placental species with superfetation indeed inhabit deeper, faster-flowing parts of the river during the day (see prediction (i)), then they should show a far more pronounced diurnal shift at dusk towards shallower, slow-flowing microhabitats compared to species that lack both reproductive adaptations. (iii) Finally, we expect that juveniles and immatures of all five species tend to avoid the faster-flowing sections of the river, because their swimming abilities are still limited (Dial et al., 2016; Lankheet et al., 2016). If true, then these younger ontogenetic stages (juveniles and to a lesser extent immatures) should display a similar habitat selection regardless of the species, with all preferring shallow, slow-velocity areas near the riverbank. By comparing microhabitat use of these five sympatric live-bearing fish species, our study provides new insights into the potential effects of reproductive adaptations on microhabitat selection and local diurnal and ontogenetic migration.

\subsection{Material and methods}

\subsubsection{Study species}

P. retropinna, P. paucimaculata, P. turrubarensis, P. gillii, and B. roseni all inhabit freshwater streams in the Rio Terraba and Rio Coto drainages in the province of Puntarenas, Costa Rica (Bussing, 2002). With the exception of Brachyrhaphis sp., which mainly feed on ants and tiny dipterans (Pichler \& Schiemer, 2008), all study species browse on detritus, ooze, and algae (Bussing, 2002). However, the study species differ in the absence/presence (or degree) of two reproductive adaptations: placentation and superfetation (Table 4.1). The degree of placentation is quantified as the ratio of offspring mass at birth to egg mass at fertilization, also referred to as the Matrotrophy Index (MI) (Reznick et al., 2002; Pollux et al., 2014). Some live-bearing species, known as lecithotrophs (yolkfeeding), allocate all resources to eggs prior to fertilization in the form of large fully-yolked eggs. Embryos subsequently lose dry mass over the course of gestation due to metabolic processes. Such species have an MI less than 1. Other live-bearing species, known as matrotrophs (mother-feeding), allocate nutrients to the developing offspring post-fertilization throughout pregnancy. Such species have an MI greater than 1, indicating embryos gain dry mass during pregnancy. Placentotrophy represents one specific type of matrotrophy that is achieved through a follicular placenta, roughly an analog to the mammalian placenta (Pollux et al., 2009). The degree of superfetation is the number of broods at various developmental stages that are carried by a female (Turner, 1937). Females with superfetation tend to produce smaller broods, but do so more often (Reznick \& Miles, 1989). 
$P$. retropinna and $P$. paucimaculata are both characterized by superfetation and postfertilization maternal provisioning (i.e. matrotrophy). P. retropinna females carry up to four broods at various developmental stages and offspring increase in dry mass more than 100-fold during pregnancy $(\mathrm{MI}=117)$ (Reznick et al., 2002). P. paucimaculata females carry up to two broods at different stages and the embryos increase approximately 10-fold in dry mass during gestation $(\mathrm{MI}=7.8)$ (Reznick et al., 2002). P. turrubarensis exhibits superfetation, carrying up to four different broods (Zúñiga-Vega et al., 2007), but does not provide nutrients to the embryos after fertilization (i.e. $\mathrm{MI}<1$ ). In contrast, P. gillii and $B$. roseni are both non-placental species and carry embryos of only a single brood (i.e. no superfetation) (Pollux et al., 2009, 2014). All five live-bearing fish species commonly co-occur with G. maculatus, a benthic piscivorous predator found on the Pacific versant of Costa Rica (Bussing, 2002).

Table 4.1. Summary of the reproductive modes among the study species.

\begin{tabular}{lccccl}
\hline Species & Placentation & $\begin{array}{c}\text { Matrotrophy } \\
\text { Index }\end{array}$ & Superfetation & $\begin{array}{c}\text { Degree of } \\
\text { superfetation }\end{array}$ & Reference \\
\hline $\begin{array}{l}\text { Poeciliopsis } \\
\text { retropinna }\end{array}$ & $\checkmark$ & 117 & $\checkmark$ & 4 & $\begin{array}{l}\text { Reznick et al. 2002; } \\
\text { Hagmayer et al. 2018 }\end{array}$ \\
$\begin{array}{l}\text { Poeciliopsis } \\
\text { paucimaculata }\end{array}$ & $\checkmark$ & 7.8 & $\checkmark$ & 2 & Reznick et al. 2002 \\
$\begin{array}{l}\text { Poeciliopsis } \\
\text { turrubarensis }\end{array}$ & $\boldsymbol{X}$ & $<1$ & $\checkmark$ & 4 & Zúñiga-Vega et al. 2007 \\
$\begin{array}{l}\text { Poecilia gillii } \\
\text { Brachyrhaphis }\end{array}$ & $\mathbf{X}$ & $<1$ & $\boldsymbol{X}$ & 1 & Pollux et al. 2009 \\
roseni & $<$ & $<1$ & $\boldsymbol{X}$ & 1 & Pollux et al. 2009 \\
\hline
\end{tabular}

\subsubsection{Underwater visual census}

In February and March (i.e. end of dry season) 2017-2019, we selected ten sites in the province of Puntarenas in Costa Rica. The average monthly precipitation at the study sites in February and March is low ( $80 \mathrm{~mm}$; Fick \& Hijmans 2017), and thus, variation in water depth and velocity can be expected to be relatively small. At each site, 7-17 transects of 4-24 $\mathrm{m}$ were placed using two $5 \mathrm{~mm}$ ropes separated by $1 \mathrm{~m}$ (Fig. S.4.1). Each transect was divided in quadrats of $1 \mathrm{~m}$, which results in 1406 total quadrats of 1 $\mathrm{m} \times 1 \mathrm{~m}$, an average of 141 quadrats per site (Table 4.2,4.3). Underwater visibility was high at all study sites. We recorded the occupancy (i.e. presence or absence) of juveniles, immatures, and adults of all study species including G. maculatus during daytime in each quadrat of each transect. Specifically, fish occurring in deep-water transects in the middle of the river were identified by means of underwater visual census while snorkeling (Pollux et al., 2007). Specifically, the snorkeler began at the downstream end of the transect and slowly worked his way upstream, meter by meter, while recording the occupancy (i.e. presence or absence) of each species and size class after completing each meter mark (Fig. 
S.4.1). In very shallow transects (too shallow to snorkel), fish were instead identified from above while standing or sitting on the shore. Poeciliid fish can be closely approached by a snorkeler without being disturbed (e.g. without scaring them away or altering feeding behavior). All individuals were classified into three categories based on their ontogenetic stage: Adults (A), defined as large (potentially pregnant) females and mature males (fully developed gonopodium present); immatures (I), defined as small (non-pregnant) females and males that did not have fully developed gonopodia; and juveniles $(\mathrm{J})$, defined as fish $<2 \mathrm{~cm}$.

Table 4.2. Study locations (plus coordinates), study species present (PR: Poeciliopsis retropinna; PP: Poeciliopsis paucimaculata; PT: Poeciliopsis turrubarensis; PG: Poecilia gillii; BR: Brachyrhaphis roseni; GM: Gobiomorus maculatus), census dates, and number of transects $\left(n_{t}\right)$ at each site.

\begin{tabular}{|c|c|c|c|c|}
\hline River & Species & Coordinates & Census date & $n_{t}$ \\
\hline Rio Pedregoso & PR, PP & $\mathrm{N} 09^{\circ} 21^{\prime}, \mathrm{W} 83^{\circ} 43^{\prime}$ & 12.03 .2017 & 7 \\
\hline Rio Balsar & $P R, P G, G M$ & N $08^{\circ} 59^{\prime}, W 83^{\circ} 31^{\prime}$ & 27.03 .2018 & 11 \\
\hline Rio Tinoco & $P R, P G, B R$ & N $08^{\circ} 54^{\prime}, W 83^{\circ} 22^{\prime}$ & 27.03 .2018 & 9 \\
\hline Rio Corredor & $\mathrm{PR}, \mathrm{BR}$ & $\mathrm{N} 08^{\circ} 40^{\prime}, \mathrm{W} 82^{\circ} 54^{\prime}$ & 28.03 .2018 & 14 \\
\hline Rio Esquinas & PR, PP & $\mathrm{N} 08^{\circ} 44^{\prime}, \mathrm{W} 83^{\circ} 10^{\prime}$ & 29.03 .2018 & 15 \\
\hline Rio Union (downstream) & $P R, P P, P G$ & N $09^{\circ} 15^{\prime}, W 83^{\circ} 34^{\prime}$ & 09.02 .2019 & 17 \\
\hline Rio Nuevo & $\mathrm{PT}, \mathrm{GM}$ & $\mathrm{N} 08^{\circ} 31^{\prime}, \mathrm{W} 83^{\circ} 21^{\prime}$ & 12.02 .2019 & 16 \\
\hline Rio Agujas & $\mathrm{PR}, \mathrm{PT}, \mathrm{PG}, \mathrm{BR}, \mathrm{GM}$ & N $08^{\circ} 34^{\prime}, W 3^{\circ} 23^{\prime}$ & 13.02.2019 & 17 \\
\hline Rio Union (upstream) & $P P, P G$ & N $09^{\circ} 17^{\prime}$, W $83^{\circ} 32^{\prime}$ & 14.02 .2019 & 16 \\
\hline Rio Pacuar & $P R, P P, P G$ & $\mathrm{~N} 09^{\circ} 21^{\prime}, \mathrm{W} 83^{\circ} 44^{\prime}$ & 15.02 .2019 & 15 \\
\hline Rio Corredor & $\mathrm{PR}, \mathrm{BR}$ & $\mathrm{N} 08^{\circ} 40^{\prime}, \mathrm{W} 82^{\circ} 54^{\prime}$ & 19.02 .2019 & 6 \\
\hline Total & & & & 143 \\
\hline
\end{tabular}

Immediately following the daytime census, we measured the (i) water depth to the nearest cm by using an aluminum meter stick and (ii) water velocity to the nearest $0.01 \mathrm{~m} \cdot \mathrm{s}^{-1}$ with a Höntzsch Vane Wheel FA current meter (type ZS30 GFE md20 T/100-2/p10, Höntzsch Instruments, Waiblingen, Germany) three times separately in the center of each $1 \times 1 \mathrm{~m}$ quadrat. In quadrats where the water depth exceeded $60 \mathrm{~cm}$, the water velocity was calculated as the average between the velocities measured at 0.2 times the water depth and 0.8 times the water depth in the center of the quadrat (Hauer \& Lamberti, 2007). This ultimately yielded a mean water velocity for each $1 \times 1 \mathrm{~m}$ quadrat, calculated as the average of the three (or six if water depth exceeded $60 \mathrm{~cm}$ ) repeated measurements in its center.

Finally, to quantify diurnal (day-night) shifts in microhabitat use, each transect was censused a second time that same night (as described above) (Fig. S.4.1). For this, we used a 2000 Lumen ThorFire S1 underwater lamp to identify fish occurring in deep-water transects while snorkeling and headlamps for identifying fish in the shallow shore transects. To ensure the same transect positions between day and night census, the transects placed 
during the day were left in place until we returned the same night.

Table 4.3. Study locations, mean and range of water depth $(\mathrm{m})$ and velocity $\left(\mathrm{m} \cdot \mathrm{s}^{-1}\right)$, number of quadrats $\left(n_{q}\right)$, and percentage of occupied quadrats at day and night at each site.

\begin{tabular}{lccccc}
\hline River & $\begin{array}{c}\text { mean depth } \\
{[\mathrm{min}, \mathrm{max}]}\end{array}$ & $\begin{array}{c}\text { mean velocity } \\
{[\mathrm{min}, \mathrm{max}]}\end{array}$ & $\begin{array}{c}n_{q} \\
\% \text { occupied } \\
\text { day }\end{array}$ & $\begin{array}{c}\text { \% occupied } \\
\text { night }\end{array}$ \\
\hline Rio Pedregoso & $0.26[0.04,0.60]$ & $0.23[0.00,1.28]$ & 98 & 20 & 44 \\
Rio Balsar & $0.37[0.05,1.20]$ & $0.10[0.00,0.78]$ & 110 & 48 & 40 \\
Rio Tinoco & $0.26[0.05,0.55]$ & $0.05[0.00,0.37]$ & 73 & 95 & 88 \\
Rio Corredor & $0.20[0.05,0.60]$ & $0.20[0.00,1.03]$ & 133 & 89 & 62 \\
Rio Esquinas & $0.29[0.01,0.80]$ & $0.37[0.00,1.35]$ & 132 & 40 & 31 \\
Rio Union (downstream) & $0.38[0.05,0.80]$ & $0.16[0.00,0.95]$ & 169 & 75 & 31 \\
Rio Nuevo & $0.28[0.05,0.95]$ & $0.21[0.01,0.76]$ & 160 & 61 & 26 \\
Rio Agujas & $0.24[0.05,0.65]$ & $0.22[0.00,0.89]$ & 168 & 61 & 40 \\
Rio Union (upstream) & $0.29[0.05,0.85]$ & $0.21[0.00,0.78]$ & 158 & 65 & 44 \\
Rio Pacuar & $0.20[0.01,0.55]$ & $0.11[0.00,0.58]$ & 150 & 79 & 64 \\
Rio Corredor & $0.23[0.05,0.80]$ & $0.11[0.00,0.42]$ & 55 & 93 & 75 \\
\hline Total & & 1406 & & \\
\hline
\end{tabular}

\subsubsection{Data analysis}

The habitat preference by the different species was quantified using the Bayesian programming environment JAGS (Plummer, 2003) in R v 3.5 (R Core Team, 2020).

For this, we quantified (i) the occupancy probability of a given age-class of a species as a function of water depth and velocity, as well as (ii) the preferred mean water depth and velocity for a given age-class of a species.

For (i), the occupancy (i.e. presence or absence) of a given age-class of a species per quadrat was fitted in a Bernoulli generalized linear mixed model as a function of the three-way interaction between water depth, age, and day-night cycle, and the three-way interaction between water velocity, age, and day-night cycle. Additional variables included the second order polynomials of water depth and velocity. The model estimates species-specific slopes on each of the parameters and quadrat-, transect-, and site-specific random intercepts to account for pseudo-replication and between-transect/site variation, respectively. In the case of G. maculatus, information about 'age' is not available. Thus, occupancy per quadrat was fitted as a function of the two-way interaction between water depth and day-night cycle, and the two-way interaction between water velocity and daynight cycle. The quadrat-, transect-, and site identity were fitted as additional intercepts (see above), and the second order polynomials of water depth and velocity as additional slopes.

For (ii), the water depth and velocity of the occupied quadrats only (i.e. quadrats with occupancy $=1$ ) were fitted as a function of the three-way interaction between age, daynight cycle, and species in a bivariate Gaussian model allowing for the covariance between 
the residuals of both responses. In the case of $G$. maculatus, the water depth and velocity of the occupied quadrats were fitted in a bivariate Gaussian model as a function of the day-night cycle only.

Convergence was assessed by visual examination of the traces and by checking that $\hat{R}<1.01$. The model fits were assessed using posterior predictive checks on the model predictions. The priors, number of MCMC chains, iterations, burnin, and thinning is given in the Supplementary Methods S.4.1.1.

\subsection{Results}

\subsubsection{Ontogenetic microhabitat use during daytime}

During the day, adults of all species occupied deeper, faster-flowing water than immatures, and immatures occupied deeper, faster-flowing water than juveniles (Table S.4.1; Fig. 4.1: left panels, Fig. 4.2). This ontogenetic habitat use was most pronounced in P. retropinna and P. paucimaculata, intermediate in P. turrubarensis, and least obvious in P. gillii and B. roseni (Table S.4.2, S.4.3; Fig. 4.1: left panels). In other words, adults of species that have a placenta and superfetation ( $P$. retropinna and $P$. paucimaculata) tend to inhabit deeper, faster-flowing parts in the middle of the river compared to adults of species that lack both reproductive adaptations ( $P$. gillii and B. roseni). P. turrubarensis which has superfetation but lacks a placenta inhabits intermediate water depth and velocity. Moreover, juveniles (and to a lesser extent immatures) display a similar habitat selection regardless of the species. Specifically, they prefer shallow, slow-velocity areas near the riverbank (Table S.4.1; Fig. 4.1: left panels). 

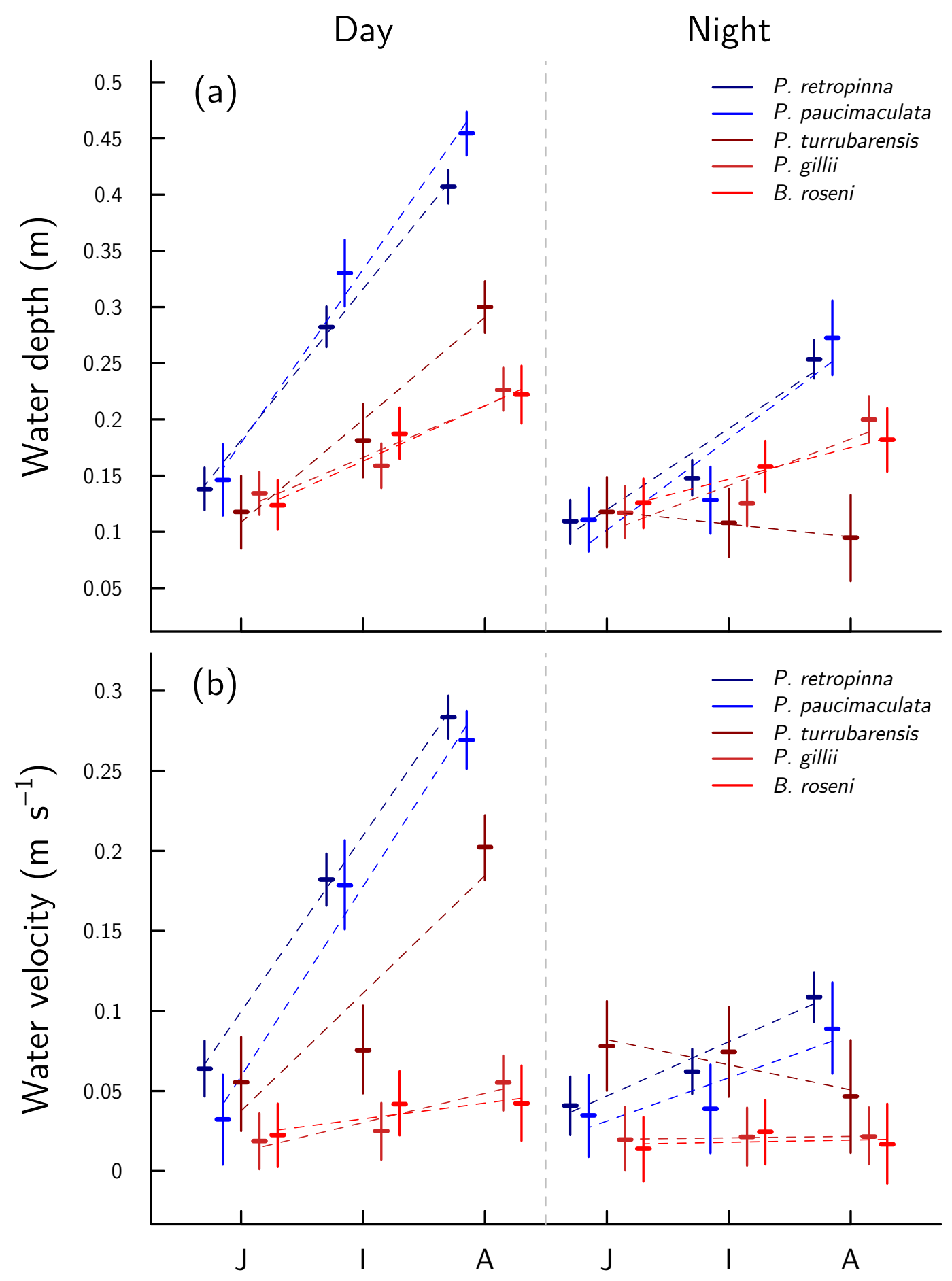

Fig. 4.1. (a) Mean water depth and (b) velocity ( $\pm 95 \%$ posterior density $\mathrm{Cl}$ ) occupied by a given age-class of a species during day and night. Dotted line corresponds to a linear fit throughout the posterior samples of a given species at day or night, respectively. J: juvenile, I: immature, A: adult. Blue: placental species with superfetation; brown: non-placental species with superfetation; red: non-placental species without superfetation. 


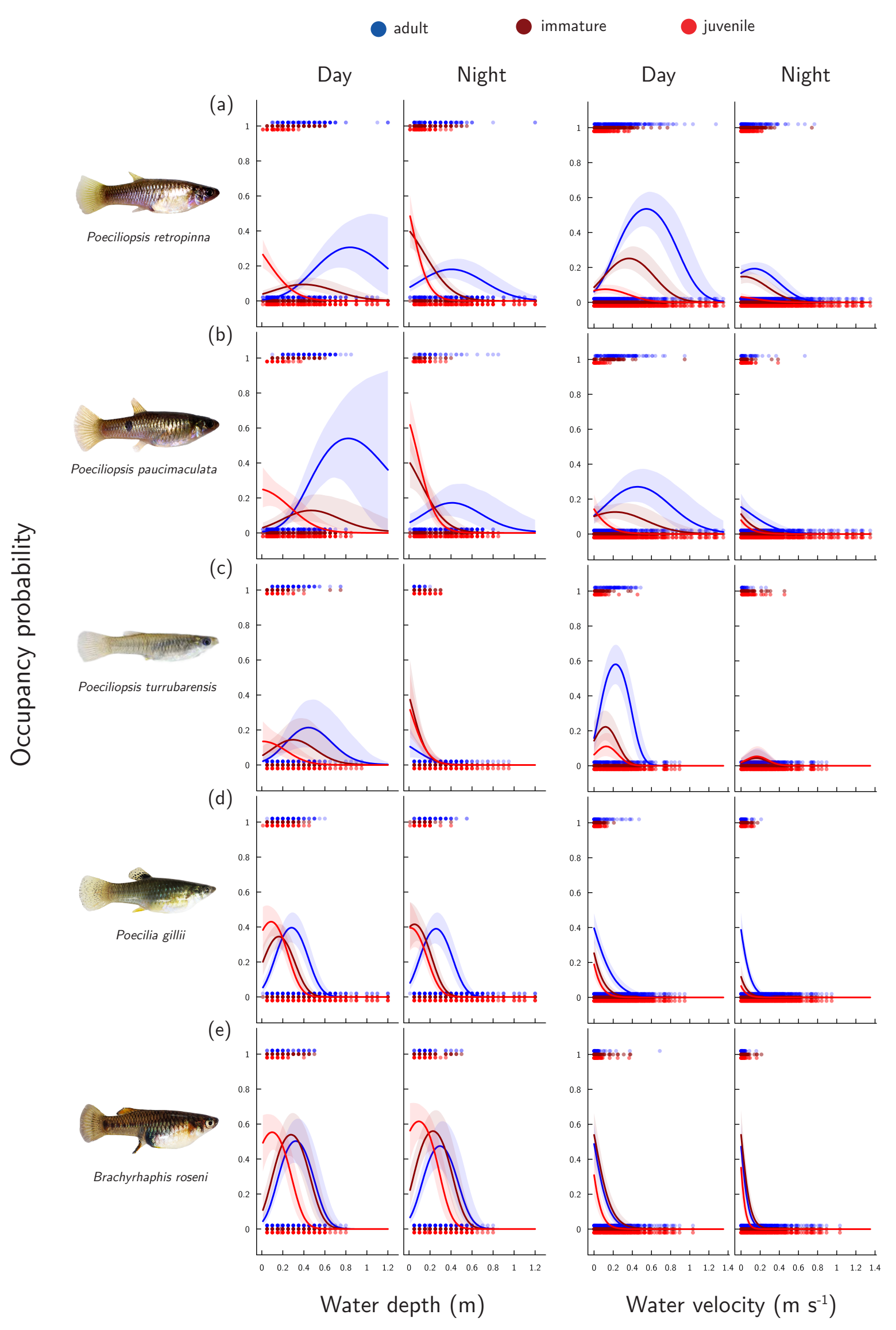


Fig. 4.2. Occupancy probability ( $\pm 95 \%$ posterior density $\mathrm{Cl}$ ) of a given age-class of a species as a function of water depth (water velocity kept constant at zero), velocity (water depth kept constant at mean, i.e. $0.29 \mathrm{~m}$ ), and day-night cycle (left panels: day; right panels: night). red: juvenile, brown: immature, blue: adult. (a) Poeciliopsis retropinna, (b) Poeciliopsis paucimaculata, (c) Poeciliopsis turrubarensis, (d) Poecilia gillii, and (e) Brachyrhaphis roseni. Data points correspond to the raw data (i.e. whether a quadrat is occupied by a given age-class of a species or not).

\subsubsection{Ontogenetic microhabitat use disappears at night}

At night, the ontogenetic microhabitat use observed during daytime disappears. At night fall, all age-classes, regardless of the species, either remain in or move towards the shallow river shore where they congregate in the slow-flowing waters presumably to sleep (Table S.4.1; Fig. 4.1: right panels, Fig. 4.2). As a result, adults of species that have a placenta and superfetation ( $P$. retropinna and $P$. paucimaculata) show a far more pronounced diurnal (day-night) migration towards shallower, slow-flowing microhabitats compared to species that lack both reproductive adaptations ( $P$. gillii and B. roseni). P. turrubarensis which has superfetation but lacks a placenta displays an intermediate diurnal microhabitat shift (Table S.4.2, S.4.3; Fig. 4.1). Interestingly, the piscivorous ambush predator Gobiomorus maculatus likewise moves to the slower-flowing and shallower areas at night (depth: $\beta_{\text {post.mean }}=0.111,95 \% \mathrm{CI}=0.046-0.175, P_{\mathrm{MCMC}}=0.002$; velocity: $\beta_{\text {post.mean }}=0.063,95 \% \mathrm{CI}=0.005-0.118, P_{\mathrm{MCMC}}=0.035 ;$ Fig. $\left.4.3,4.4\right)$.
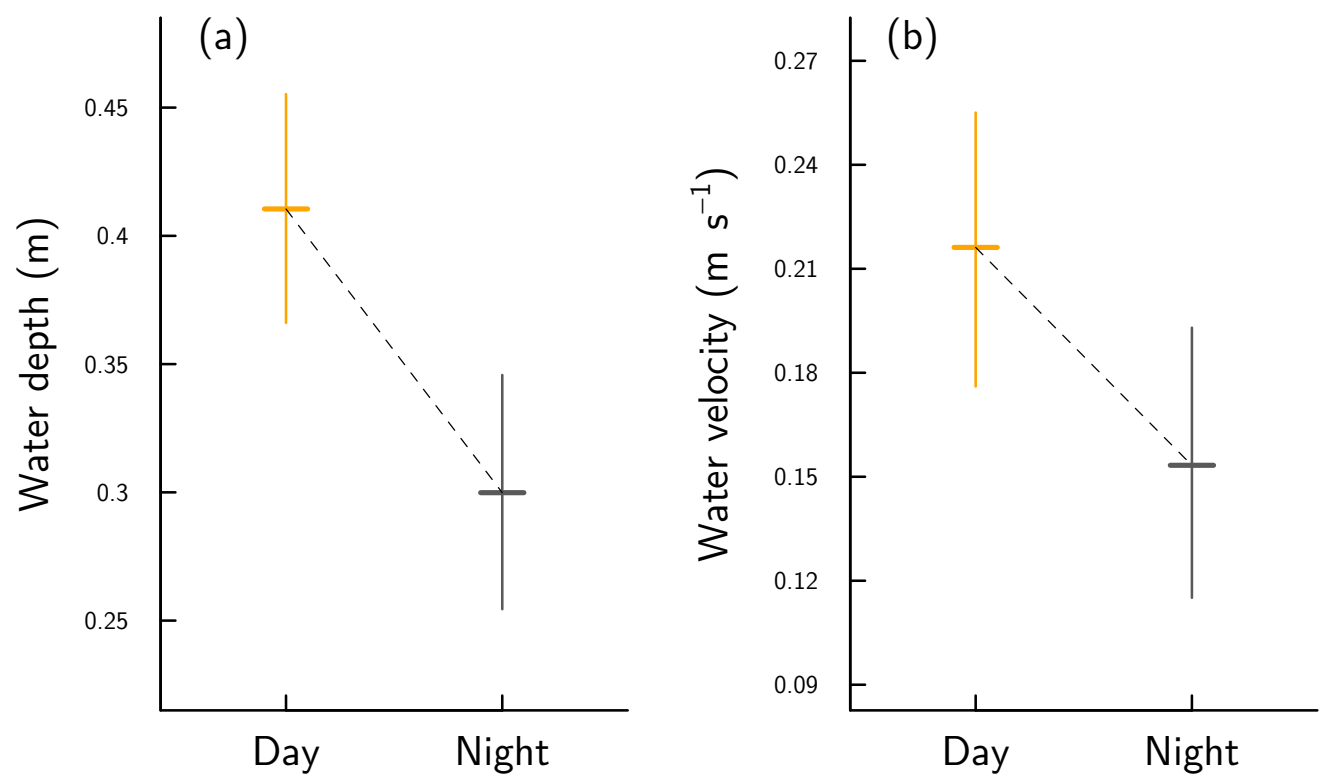

Fig. 4.3. (a) Mean water depth and (b) velocity ( $\pm 95 \%$ posterior density $\mathrm{Cl}$ ) occupied by the predator species Gobiomorus maculatus during day (yellow) and night (grey). Dotted line corresponds to a linear fit throughout the posterior samples of the day-night cycle. 


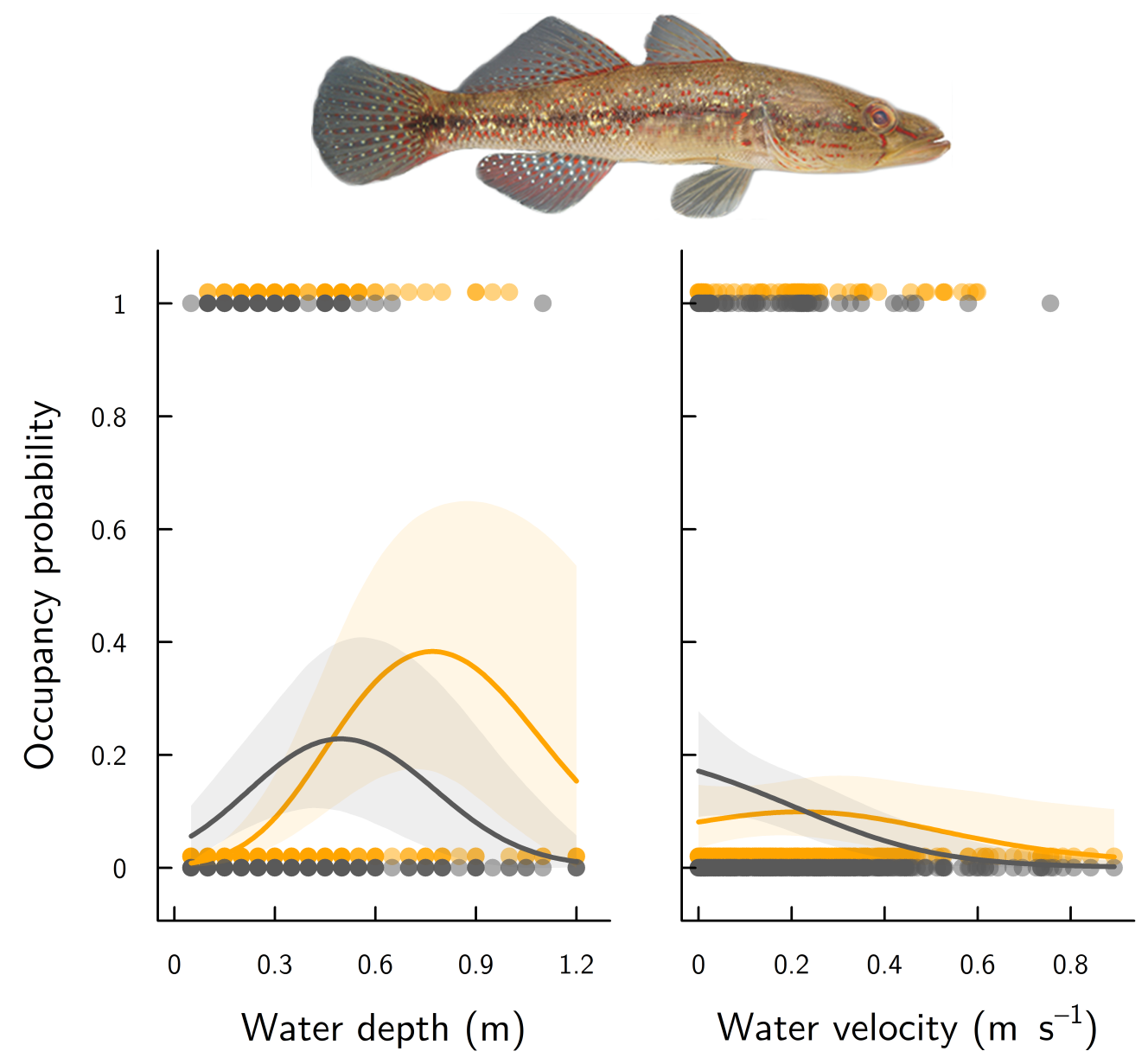

Fig. 4.4. Occupancy probability $( \pm 95 \%$ posterior density $\mathrm{Cl})$ of the piscivorous predator fish Gobiomorus maculatus as a function of water depth (left panel; water velocity kept constant at zero), velocity (water depth kept constant at mean, i.e. $0.28 \mathrm{~m}$; right panel), and day-night cycle (yellow: day; grey: night). Data points correspond to the raw data (i.e. whether a quadrat is occupied by a given age-class of a species or not). Photo: Fredy Nugra.

\subsection{Discussion}

\subsubsection{Ontogenetic (age-related) microhabitat use during daytime}

We found that adults generally tend to prefer faster-flowing and deeper water during the day than immatures, and that immatures prefer faster-flowing and deeper water than juveniles. This is most likely due to the positive association between fish size (and thus, indirectly, age or ontogenetic stage; Reznick et al. 1996a) and swimming capability in Teleost fish (Gibb et al., 2006). Newborn poeciliid fish have relatively poor swimming abilities (Dial et al., 2016; Lankheet et al., 2016). They are physically incapable of maintaining their position in fast-flowing water and thus seek refuge in low-velocity areas of 
the river near the shore. An added benefit for these small juveniles is that here they can inhabit very shallow water (sometimes less than $1 \mathrm{~cm}$ deep) where they are out of reach of piscivorous predators. The subsequent growth of fish is associated with physical ability and better control of the locomotor system (Fuiman \& Higgs, 1997; Gibb et al., 2006; Lankheet et al., 2016), leading to an increase in swimming ability with increasing body length (Gibb et al., 2006). This allows larger and physically stronger adults to inhabit the relatively faster-flowing parts of the river.

The above may explain why only adult individuals are physically able to swim in the fast flow. However, it does not explain why some would want to move to the fast-flowing parts of the river in the first place, considering that there they are exposed to high drag forces (Quicazan-Rubio et al., 2019) making it energetically expensive for them to swim. We tentatively propose that some adults may move to deeper water to avoid avian predation. Together with various other piscivorous birds (e.g. herons), the southern Pacific region of Costa Rica is inhabited by four species of kingfisher (Garrigues \& Dean, 2014), which mainly feed on fish (Fry et al., 1999). Piscivorous birds are very effective predators in shallow shore water or close to the surface (Whitfield \& Cyrus, 1978; Kramer et al., 1983), where they show a preference for larger fish (Power, 1984; Trexler et al., 1994). This means that large adult poeciliids are more vulnerable to avian predation in shallow water than small juveniles. At the same time, piscivorous fish are presumably feeding in deeper water to prevent avian predation (Power, 1984). Indeed, we found that the piscivorous predator G. maculatus inhabits relatively deeper water during the day (i.e. when most piscivorous birds are active in the shallows). It has furthermore been shown that larger prey fish are able to perform faster escape responses, making them less vulnerable to predation by piscivorous fish (Gibb et al., 2006). Thus, one could argue that the mortality risk of livebearing fish during daytime is highest for adults in shallow habitats from piscivorous birds, and highest for juveniles in deep habitats from piscivorous fish (Power, 1984; Schlosser, 1988). If true, then it would be advantageous for juveniles to inhabit shallow waters near the shore, while it would be beneficial for larger adults to move to deeper water as they outgrow the vulnerability to piscivorous fish (Power, 1984). Collectively, these findings suggest that the observed ontogenetic microhabitat preference in live-bearing fish during daytime may be an adaptive response to predation risk.

\subsubsection{Diurnal (day-night) shifts in microhabitat use}

Many fish exhibit diurnal shifts in microhabitat use (Lowe-McConnell, 1975; Helfman, 1986), which have been attributed to shifts in foraging activity (Piet \& Guruge, 1997), the use of shallow water as refuge from predation (Copp \& Jurajda, 1993; Arrington \& Winemiller, 2003) or the use of slow-flowing areas to reduce energy expenditure while resting at night (Matheney IV \& Rabeni, 1995; Sempeski \& Gaudin, 1995). In our study, we found that at dusk all fish, regardless of their ontogenetic stage (juvenile, immature or adult), tend to move to shallow waters near the shore, where they sleep lying on the bottom 
in low-velocity areas. Occasionally, large adult individuals (particularly P. retropinna and P. paucimaculata) can be found sleeping while wedged into crevices or behind stones in deeper and faster flowing stretches of the river. Interestingly, G. maculatus, the most common piscivorous fish species in our study sites, is primarily a nocturnal sit-and-wait bottom predator (Swing, 1992). The finding that this piscivorous predator also moves towards shallow water at night, suggests that the observed diurnal microhabitat shifts in poeciliid fish towards shallow water are not related to predator avoidance. Instead, it is more likely that the day-night shifts are driven by a preference for low-velocity areas in the river to avoid being washed away while resting at night.

\subsubsection{Differences in diurnal and ontogenetic microhabitat use among poeciliid species with different reproductive adaptations}

All five studied poeciliid species occur sympatrically in freshwater streams in Costa Rica (Bussing, 2002), yet show remarkable differences in ontogenetic and diurnal microhabitat use. This appears to be correlated with the absence/presence of the two reproductive adaptations. During daytime, adult $P$. retropinna and $P$. paucimaculata (which both have a placenta and superfetation) can be found in very fast-flowing water (of up to 1.3 $\mathrm{m} \cdot \mathrm{s}^{-1}$ ), while adult $P$. gillii and B. roseni (which both lack a placenta and superfetation) are confined to shallow and slow-flowing areas. Adult $P$. turrubarensis (which lacks a placenta but has superfetation) notably prefers an intermediate habitat. At night these differences in ontogenetic microhabitat segregation among species disappear when all ontogenetic stages (juveniles, immatures, and adults) of all five species move towards shallow waters presumably to sleep in low-velocity areas.

This raises the question why the adults of $P$. retropinna and $P$. paucimaculata, and to a somewhat lesser extent of $P$. turrubarensis are found in fast-flowing water during daytime? It has been argued that the evolution of placentation and superfetation both reduce a female's reproductive burden during pregnancy, yet achieve this in fundamentally different ways (Thibault \& Schultz, 1978; Pollux et al., 2009; Pires et al., 2011). The evolution of the placenta is associated with a shift in the timing of maternal provisioning from pre- to post-fertilization. Non-placental live-bearers (e.g. P. gillii, B. roseni, and P. turrubarensis) typically produce large fully-yolked eggs, committing all the nutrients required for embryo development to the egg prior to fertilization. Placental species ( $P$. retropinna and $P$. turrubarensis), by contrast, produce relatively small eggs and instead provide most nutrients to their offspring throughout pregnancy via a placenta (Wourms, 1981; Pollux et al., 2009). The shift in the timing of maternal provisioning from pre- to postfertilization reduces a female's reproductive burden (Reznick et al., 2007; Bassar et al., 2014; Fleuren et al., 2018). The evolution of superfetation furthermore correlates with the more frequent production of smaller broods (Reznick \& Miles, 1989). By spreading reproduction more evenly over time, superfetation is thought to reduce a female's peak reproductive allotment during gestation without reducing maternal fecundity (Pollux et al., 
2009). Thus, placentation and superfetation are both thought to reduce a female's reproductive burden during pregnancy (Thibault \& Schultz, 1978; Reznick et al., 2007; Pollux et al., 2009; Pires et al., 2011; Bassar et al., 2014; Hagmayer et al., 2020c). This is likely to cause a more slender body shape (Zúñiga-Vega et al., 2007; Fleuren et al., 2018, 2019), reduced body drag (Quicazan-Rubio et al., 2019), as well as improved sustained swimming performance (Plaut, 2002) and fast-start escape response (Ghalambor et al., 2004; Fleuren et al., 2019). Thus, one might argue that placentation and superfetation are reproductive adaptations that facilitate the utilization of high 'performance-demanding' microhabitats (e.g. high-flow areas) in the river. Our study provides the first empirical evidence in support of one aspect of this hypothesis, namely the idea that placentation and superfetation are reproductive adaptations that can drive differences in ontogenetic, diurnal, and reproductive microhabitat use between sympatric live-bearing species.

\subsection{Conclusion}

We report large differences in adult microhabitat use between five sympatric poeciliid species during daytime (but not nighttime). We argue that it may be selectively advantageous for large adults to move to deeper and faster-flowing water, because here the risk of avian predation (Whitfield \& Cyrus, 1978) and interspecific resource competition with other poeciliid species (Lanza, 1983) is reduced. Previous studies suggest that both placentation and superfetation improve body streamlining and hence swimming ability (Fleuren et al., 2018, 2019; Quicazan-Rubio et al., 2019). This may explain, at least in part, why adult $P$. retropinna and $P$. paucimaculata, and to a lesser extent $P$. turrubarensis, inhabit deeper, faster-flowing areas during daytime, while B. roseni and P. gillii are more confined to the shallow waters near the shore. The finding that, at night, all fish (regardless of species or age-class) move to shallow, low-velocity areas to rest, lends additional support to this idea. Collectively our results suggest that a female's reproductive strategy (e.g. placentation and superfetation) may be a hitherto unrecognized biological feature that may help to understand microhabitat preferences between sympatric live-bearing fish species. Our study can be seen as a first step on which future, ideally experimental, studies can build upon to assess the costs of locomotion as a function of reproductive mode and pregnancy state. Future studies should focus on comparing microhabitat use in more live-bearing fish (e.g. from the family Poeciliidae, Anablepidae, Goodeidae or Zenarchopteridae), but also other aquatic live-bearing animals (e.g. amphibians, reptiles, and mammals), to assess the generality of these findings. 


\section{S.4 Supplementary Information}

\section{S.4.1 Supplementary Methods}

\section{S.4.1.1 Data analysis}

The habitat preference by the different species was quantified using the Bayesian programming environment JAGS (Plummer, 2003). For this, we modeled (1) the occupancy (i.e. presence or absence) of a given age-class of a species per quadrat in a Bernoulli generalized linear mixed model, and (2) water depth and velocity of the occupied quadrats only (i.e. quadrats with occupancy $=1$ ) in a bivariate Gaussian model allowing for the covariance between the residuals of both responses. In the case of Gobiomorus maculatus, information about 'age' is not available. Thus, each model was fitted separately to poeciliid fish (1a and $2 \mathrm{a}$ ) and G. maculatus (1b and $2 \mathrm{~b}$ ).

For (1a), we used $N\left(0,5^{2}\right)$ priors for all fixed effects. The priors for the second order polynomials of water depth and velocity were furthermore restricted to be negative. For quadrat identity, we used a $N\left(0, \sigma^{2}\right)$ prior with the common variance $\sigma^{2} \sim$ Inv-Gamma(0.01,0.01), parameterized with shape and rate parameters. The transectand site-specific intercepts were estimated using $N\left(\mu, \sigma^{2}\right)$ priors, with the common mean $\mu \sim N\left(0,1^{2}\right)$ and the common standard deviation $\sigma \sim$ Student- $t_{(0, \infty)}\left(0,1^{2}, 1\right)$, parameterized with mean, variance, and degrees of freedom. Three MCMC chains were run for 75,000 iterations, with a burnin of 25,000 and a thinning of 50. Convergence was assessed by visual examination of the traces and by checking that $\hat{R}<1.01$.

For (1b), we used $N\left(0,5^{2}\right)$ priors for all fixed effects. The priors for the second order polynomials of water depth and velocity were furthermore restricted to be negative. For quadrat identity, we used a $N\left(0, \sigma^{2}\right)$ prior with the common variance $\sigma^{2} \sim \operatorname{Inv-Gamma}(0.1,0.1)$, parameterized with shape and rate parameters. The transectand site-specific intercepts were estimated using $N\left(\mu, \sigma^{2}\right)$ priors, with the common mean $\mu \sim N\left(0,1^{2}\right)$ and the common standard deviation $\sigma \sim$ Student- $t_{(0, \infty)}\left(0,1^{2}, 1\right)$, parameterized with mean, variance, and degrees of freedom. Three MCMC chains were run for $1,500,000$ iterations, with a burnin of 500,000 and a thinning of 1000 . Convergence was assessed by visual examination of the traces and by checking that $\hat{R}<1.01$.

For $(2 \mathrm{a}$ and $2 \mathrm{~b})$, we used $N\left(0,5^{2}\right)$ priors for all fixed effects. For the residual (co)variances we used Student- $t_{(0, \infty)}\left(0,1^{2}, 1\right)$ priors, parameterized with mean, variance, and degrees of freedom. Finally, we used a $N_{(-1,1)}\left(0,1^{2}\right)$ prior for the residual correlation between water depth and velocity. Three MCMC chains were run for 1,500,000 iterations, with a burnin of 500,000 and a thinning of 1000 . Convergence was assessed by visual examination of the traces and by checking that $\hat{R}<1.01$. 


\section{S.4.2 Supplementary Results}

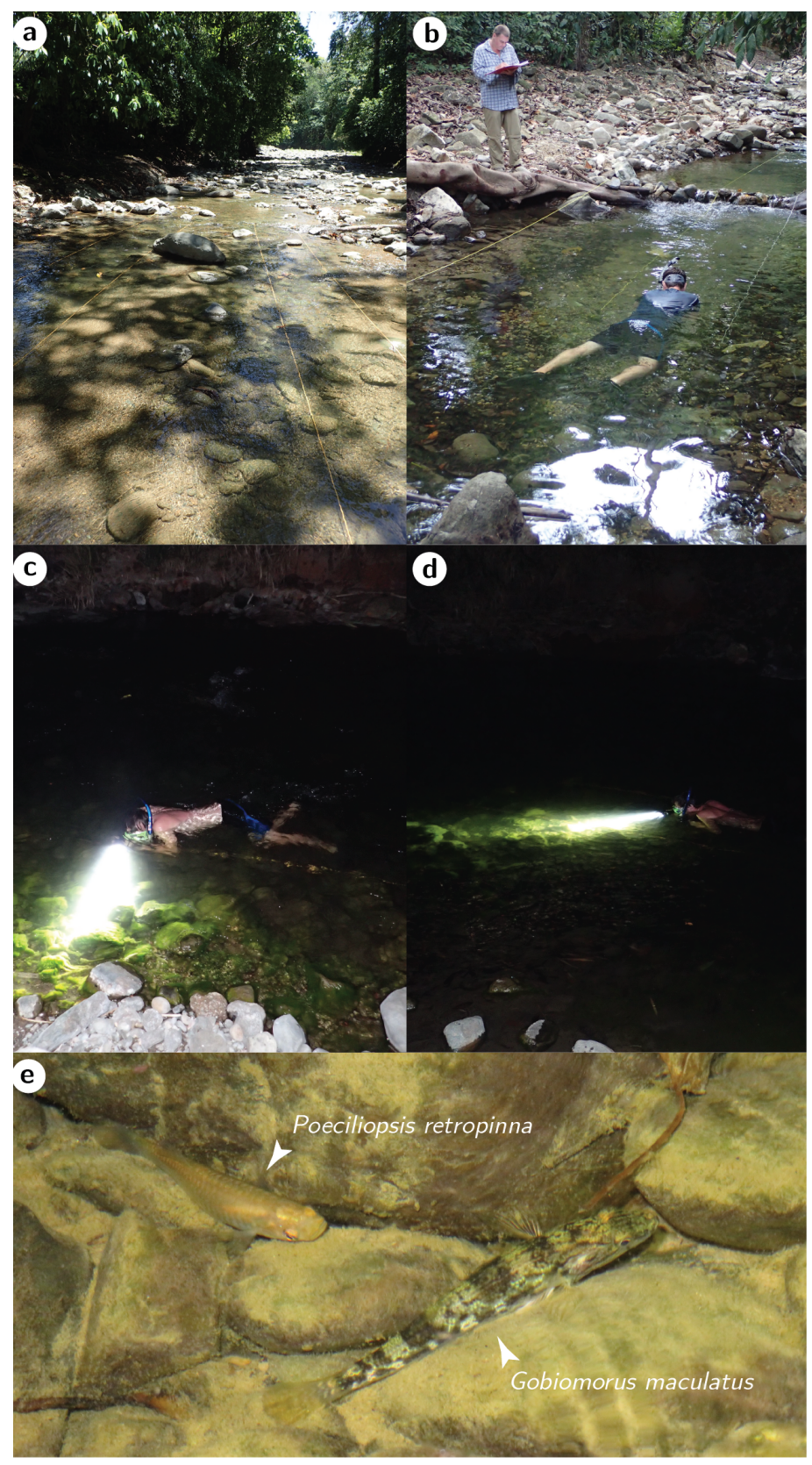

Fig. S.4.1. a Example of two transects at Rio Corredor. Each transect is 4-24 $\mathrm{m}$ long and is build with two $5 \mathrm{~mm}$ ropes separated by $1 \mathrm{~m}$. b Fish occurring in deep-water transects in the middle of the river were identified by means of underwater visual census while snorkeling. Specifically, the snorkeler began at the downstream end of the transect and slowly worked his way upstream, meter by meter, while counting the number of individuals of each species and size class to the data recorder after completing each meter mark. c and $\mathbf{d}$ night-snorkeling to quantify diurnal (day-night) shifts in microhabitat use. A 2000 Lumen ThorFire S1 underwater lamp was used to identify fish occurring in deep-water transects while snorkeling. e Adult Poeciliopsis retropinna female and its piscivorous predator Gobiomorus maculatus lying on the bottom in low-velocity areas at shore at night. 
Table S.4.1. Mean water depth and velocity ( $\pm 95 \%$ posterior density $\mathrm{Cl}$ ) occupied by a given age-class of a species during day and night. The species are summarized by their reproductive adaptation (i.e. placental species with superfetation, non-placental species with superfetation, and non-placental species without superfetation).

\begin{tabular}{lcccccc}
\hline & \multicolumn{3}{c}{ Water depth [m] } & \multicolumn{3}{c}{ Water velocity $[\mathrm{m} / \mathrm{s}]$} \\
\hline group & mean & $2.5 \% \mathrm{Cl}$ & $97.5 \% \mathrm{Cl}$ & mean & $2.5 \% \mathrm{Cl}$ & $97.5 \% \mathrm{Cl}$ \\
\hline J Day P_SF & 0.142 & 0.117 & 0.173 & 0.048 & 0.008 & 0.079 \\
I Day P_SF & 0.306 & 0.267 & 0.356 & 0.180 & 0.156 & 0.203 \\
A Day P_SF & 0.431 & 0.395 & 0.471 & 0.276 & 0.254 & 0.295 \\
J Night P_SF & 0.110 & 0.086 & 0.135 & 0.038 & 0.014 & 0.060 \\
I Night P_SF & 0.138 & 0.103 & 0.162 & 0.051 & 0.016 & 0.074 \\
A Night P_SF & 0.263 & 0.237 & 0.301 & 0.099 & 0.065 & 0.123 \\
J Day NP_SF & 0.118 & 0.085 & 0.150 & 0.055 & 0.025 & 0.084 \\
I Day NP_SF & 0.181 & 0.149 & 0.214 & 0.075 & 0.049 & 0.103 \\
A Day NP_SF & 0.300 & 0.277 & 0.323 & 0.202 & 0.182 & 0.222 \\
J Night NP_SF & 0.118 & 0.086 & 0.149 & 0.078 & 0.050 & 0.106 \\
I Night NP_SF & 0.108 & 0.078 & 0.139 & 0.074 & 0.046 & 0.103 \\
A Night NP_SF & 0.095 & 0.056 & 0.133 & 0.047 & 0.011 & 0.082 \\
J Day NP_wSF & 0.129 & 0.105 & 0.151 & 0.021 & 0.002 & 0.040 \\
I Day NP_wSF & 0.173 & 0.142 & 0.207 & 0.033 & 0.010 & 0.059 \\
A Day NP_wSF & 0.224 & 0.200 & 0.247 & 0.049 & 0.023 & 0.070 \\
J Night NP_wSF & 0.121 & 0.097 & 0.144 & 0.017 & -0.004 & 0.038 \\
I Night NP_wSF & 0.142 & 0.108 & 0.178 & 0.023 & 0.004 & 0.043 \\
A Night NP_wSF & 0.191 & 0.159 & 0.218 & 0.019 & -0.005 & 0.041 \\
\hline
\end{tabular}

J: juvenile; I: immature; $A$ : adult; P_SF: placental species with superfetation (i.e. Poeciliopsis retropinna and Poeciliopsis paucimaculata); NP_SF: non-placental species with superfetation (i.e. Poeciliopsis turrubarensis); NP_wSF: non-placental species without superfetation (i.e. Poecilia gillii and Brachyrhaphis roseni). 


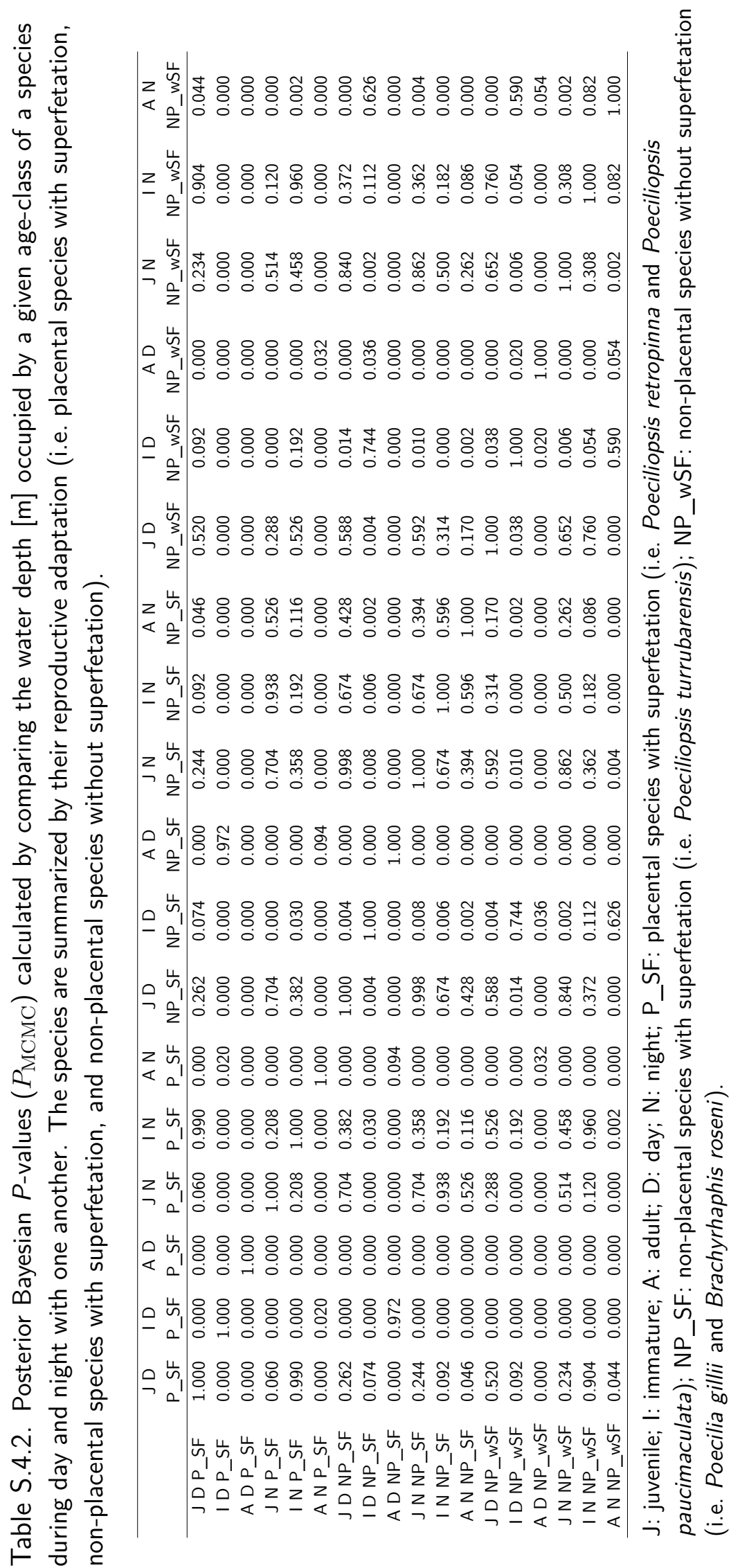




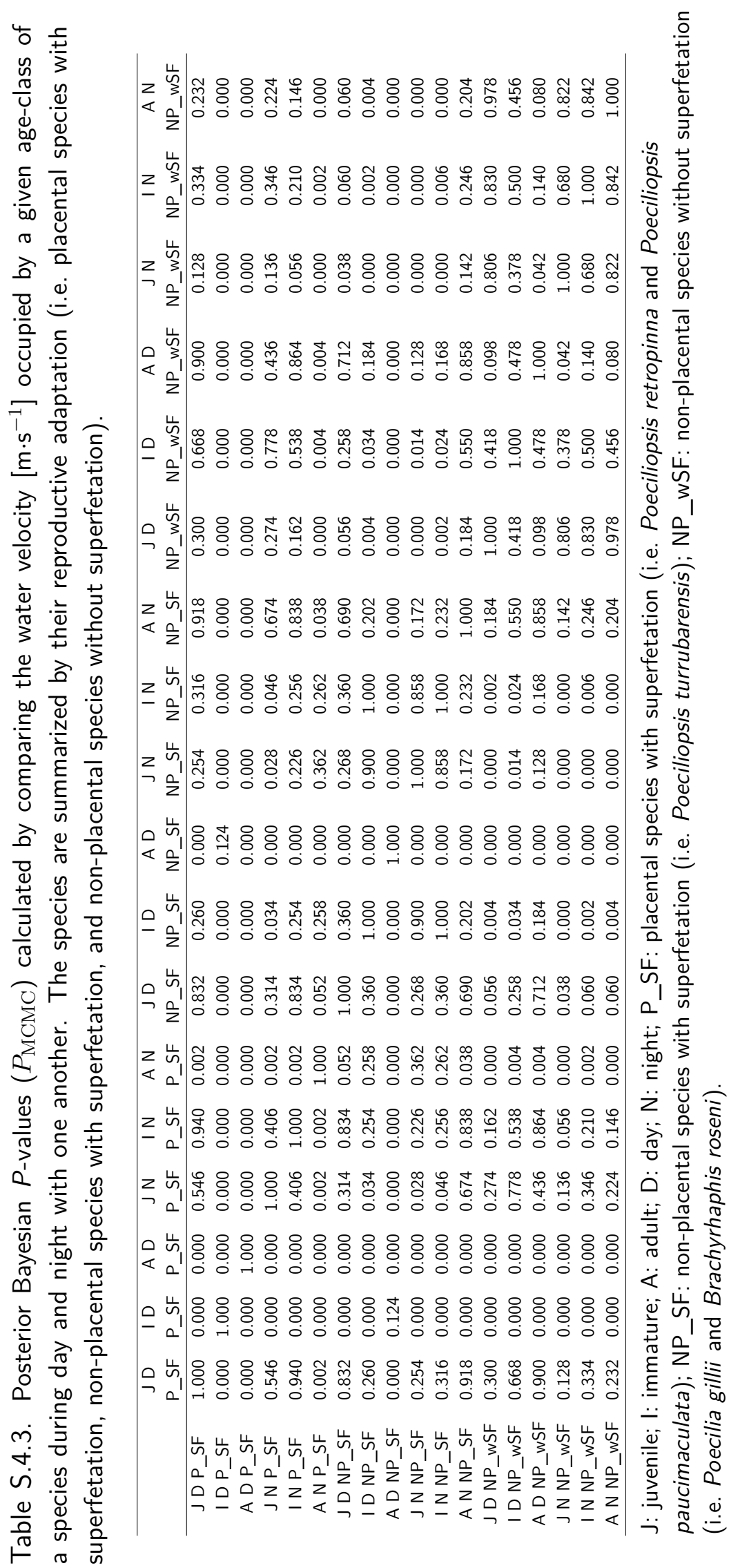




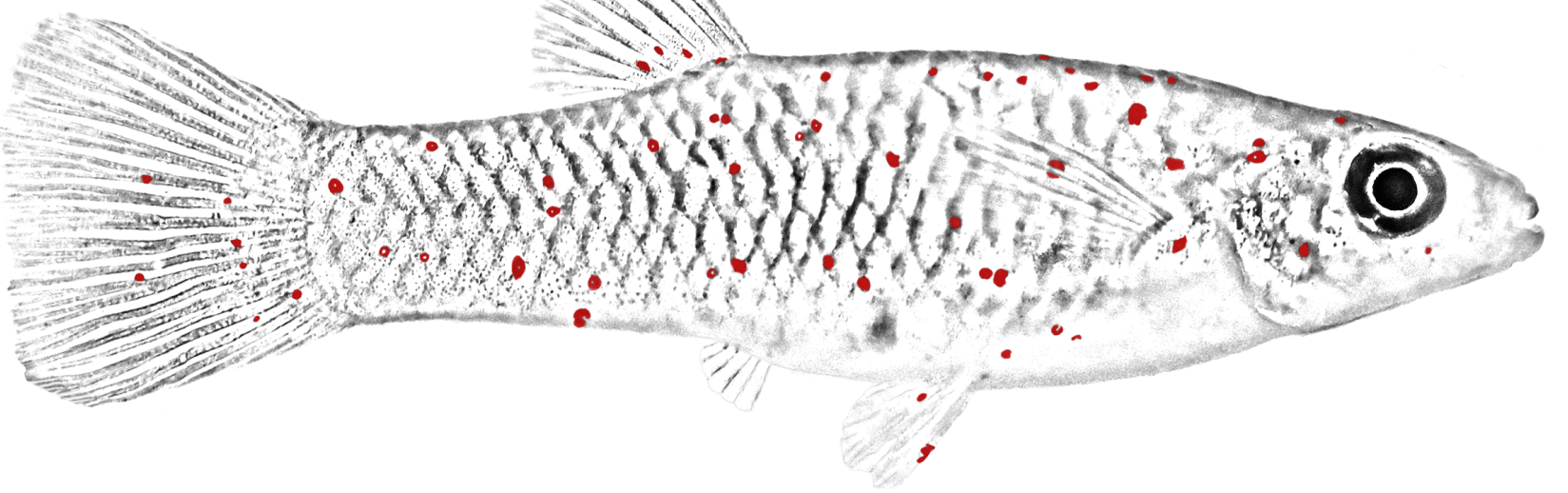




\section{Parasite infestation influences life history but not boldness behavior in placental live- bearing fish}

Andres Hagmayer ${ }^{1}$, Andrew I. Furness ${ }^{2,3} \&$ Bart J.A. Pollux ${ }^{1}$

1 Department of Animal Sciences, Wageningen University, 6708 WD

Wageningen, The Netherlands

2 Department of Ecology and Evolutionary Biology, University of California, CA 92697 Irvine, USA

3 Department of Biological and Marine Sciences, University of Hull, HU6 7RX Hull, UK

This chapter has been published as: Hagmayer, A., et al. (2020). Parasite infestation influences life history but not boldness behavior in placental live-bearing fish. Oecologia, 194:635-648. https://doi.org/10.1007/s00442-020-04795-6 


\section{Abstract}

Parasites can negatively affect the reproductive success of hosts. Placental species may be particularly susceptible, because parasite-induced stress during pregnancy could potentially influence embryo development. Here, we examine the consequences of a trematode infestation (black spot disease, BSD) for fetal development and adult behavior in 19 natural populations of the placental live-bearing fish species Poeciliopsis retropinna (Poeciliidae) in Costa Rica. First, we observed substantial variation in parasite infestation among populations which correlated with a number of local environmental conditions (elevation, river width, depth, and flow velocity). Furthermore, we observed substantial variation in parasite infestation among females within populations associated with maternal age and size. We found that the infestation rate significantly influenced embryonic development, with more heavily parasitized females producing smaller and worse-conditioned offspring at birth, possibly, because a costly immune response during pregnancy limits, either directly or indirectly, nourishment to developing embryos. Finally, a behavioral experiment in the field showed that the infestation rate did not affect an individual's boldness. Our study indicates that in placental live-bearing fish parasite infestation leads to reduced embryo provisioning during pregnancy, resulting in a smaller offspring size and quality at birth potentially with negative implications for offspring fitness.

Keywords: matrotrophy, parasites, placenta, Poeciliidae, Poeciliopsis retropinna

\subsection{Introduction}

The life history of individuals describes how resources are allocated to different functions such as maintenance, somatic growth and reproduction (Roff, 1992; Stearns, 1992). Individuals have limited resources that must be competitively allocated to these different functions. This leads to trade-offs and a limiting set of possible life-history strategies (Braendle et al., 2011). Parasites, which are ubiquitous in natural populations (Bush et al., 2001), can act on these trade-offs and induce shifts in the optimum of life-history traits (Michalakis \& Hochberg, 1994; Sheldon \& Verhulst, 1996; Richner, 1998). Therefore, studying the causes and consequences of parasite infestation is crucial for understanding the evolution of life histories.

Parasites can negatively affect the fitness of their host by directly or indirectly influencing their survival and/or reproductive success (Bush et al., 2001). Effects on survival can be the direct result of parasite-induced mortality. Soay sheep (Ovis aries) that are highly parasitized by gastrointestinal nematodes are less likely to survive the winter during periods of high overwinter mortality (Coltman et al., 1999). Moreover, lactating bighorn ewes (Ovis canadensis) were shown to be more heavily infested by lungworms compared to non-lactating ewes, and ewes that started to reproduce early in life suffered greater mortality from disease (Festa-Bianchet, 1989). Lactation (Prentice \& Prentice, 1988) and 
parasite defense both impose energetic costs on the host (Sheldon \& Verhulst, 1996), suggesting that lactating bighorn ewes are more susceptible to parasites because of the conflicting energetic demands of milk production and parasite resistance (Festa-Bianchet, 1989). Survival can also be indirectly impacted, for example by increasing the vulnerability to predators. Guppies (Poecilia reticulata) experimentally infected with a small number of cercariae of the digenean trematode Diplostomum spathaceum have been shown to be more susceptible to predation by brook trout (Salvelinus fontinalis) (Brassard et al., 1982). The increased susceptibility to predation was correlated with decreased swimming activity (measured as distance travelled per unit of time) of infected fish. Likewise, parasites can reduce the overall fecundity of hosts, either directly by affecting the nutritional status of hosts (e.g. Hurd 2001; Decaestecker et al. 2005; Tobler et al. 2005) or indirectly (e.g. by increasing offspring mortality; Brown \& Bomberger Brown 1986; Møller 1990). If parasites reduce the host's fitness, this can have profound implications for population dynamics of both hosts and parasites (Gulland, 1995).

Hosts may modify their behavior in response to a parasite infestation (Barber et al., 2000). Boldness is one of the major personality axes in animals that may be affected by parasite infestation (Barber \& Dingemanse, 2010). Boldness, defined as the propensity of an animal to engage in risky behavior, has direct implications for fecundity and survival (Sih et al., 2004). For instance, increased boldness allows individuals to forage at higher rates, leading to increased growth and/or fecundity (Sih et al., 2004). However, increased boldness also increases the mortality risk from predators, and thus, individuals must balance the conflicting demands of feeding and predator avoidance (Sih, 1980). Given that parasites can negatively affect an individual's fecundity and survival, high levels of boldness might be favored, if the compensation of the fitness costs due to predation and parasitism is efficient (Kortet et al., 2010). For instance, in sticklebacks (Gasterosteus aculeatus), individuals parasitized by cestode larvae showed increased foraging activity and recovered more quickly following an attack with a heron model (Giles, 1983). This increased activity and boldness was argued to compensate for the extra nutritional requirements caused by the parasite (Giles, 1983). Thus, parasitism may play an important role in shaping aspects of animal personalities, such as exploration, activity, or boldness (Barber \& Dingemanse, 2010).

Black spot disease (BSD) is caused by a trematode parasite (Uvulifer sp.) that is commonly found in freshwater fish (Lane \& Morris, 2010). This trematode uses aquatic snails and fish as intermediate hosts, and piscivorous birds as the final host (Hoffman \& Putz, 1965; Lane \& Morris, 2010). The sexually mature trematode produces eggs in the intestine of the bird. The eggs develop into miracidia that are released by the bird through its feces. The miracidia invade snails where they reproduce asexually and develop into freeswimming cercariae. The cercariae penetrate the skin of fish and become encapsulated by the host's tissue where they remain dormant until the fish is consumed by a piscivorous bird (Hoffman \& Putz, 1965; Tobler et al., 2007; Lane \& Morris, 2010). The penetration of the fish skin induces a melanic secretion by the host around the parasite, which forms 
externally visible black spots that are easily countable (Lively et al., 1990). Moreover, the penetration of the fish skin causes mechanical damage and hemorrhage, and the host's induction of melanocysts that enclose the parasite is expected to be energetically expensive (Lane \& Morris, 2010; Cureton et al., 2011). In line with this, BSD was shown to reduce the body condition of smallmouth bass (Micropterus dolomieui) (Hunter \& Hunter, 1938) and juvenile bluegill sunfish (Lepomis macrochiris) (Lemly \& Esch, 1984). Likewise, infected females of the live-bearing mosquitofish (Gambusia affinis) avoided shoaling with infected individuals (Tobler \& Schlupp, 2008), and females of the amazon molly (Poecilia formosa) avoided infected males (Tobler et al., 2006), which suggests some costs of associating with infected conspecifics (but see Poecilia latipinna and Poecilia mexicana in Tobler et al. 2006). Notably, however, these studies were done on egg-laying (oviparous) or egg-carrying live-bearing (lecithotrophic viviparous) fish species. The potential implications of BSD for behavior, embryo development, and quality of offspring in placental live-bearing fish remain poorly understood.

Here, we study the consequences of BSD for life-history variation and boldness in a livebearing fish species, Poeciliopsis retropinna (family Poeciliidae, Regan 1908), from Costa Rica. In this species, females transfer nutrients to their developing embryos via a 'follicular placenta', a structure that is analogous to the mammalian placenta (Pollux et al., 2009). Placentas form a physical interface between mother and fetus, allowing for intimate maternal-fetal interactions (e.g. respiration, nutrition, removal of waste products) that are crucial for normal (healthy) embryonic development. However, this intimate link also poses a risk, because maternal exposure to adverse environmental conditions (e.g. malnutrition, parasite infestation) may have unfavorable consequences for fetal development. For example, maternal parasite infestation can affect fetal growth in two non-mutually exclusive ways: (i) directly, through infestation of the developing fetus by parasites that can cross the placental barrier; and (ii) indirectly, through the modification of maternal physiology or metabolism to such an extent that it interferes with fetal development. The influence of parasite infestation on embryo development has primarily been studied in mammals (e.g. Andrews \& Lanzer 2002; Torrico et al. 2004; Gibney et al. 2008). However, placentas have evolved many times throughout the animal kingdom (e.g. Wourms 1981; Blackburn 2015; Wake 2015), including in the family Poeciliidae (Reznick et al., 2002; Pollux et al., 2014; Furness et al., 2019), yet the consequences of parasite infestation for offspring development, life-history traits, and behavior in non-mammalian placental lineages are currently insufficiently understood. P. retropinna has a particularly well-developed placenta (i.e. embryos can undergo an over 100-fold weight gain during gestation; Reznick et al. 2002) and is often found in well-defined populations (Hagmayer et al., 2020c) making it an ideal system to study the consequences of parasitism in natural populations.

To study the causes and consequences of BSD in P. retropinna, we (i) quantify the intensity of parasite infestation by scoring the number of black spots on preserved adult female $P$. retropinna, (ii) examine potential environmental predictors of parasite infesta- 
tion among populations, (iii) identify potential maternal predictors of parasite load within populations, and (iv) relate parasite load within populations to maternal life-history traits (egg mass at fertilization, offspring mass at birth, proportion of egg and offspring fat, reproductive allotment, average brood size, fecundity, superfetation, and abortion incidence) to evaluate potential costs of parasitism. Finally, we (iv) explore whether these costs influence an individual's behavior by assessing its boldness (boldness score and hesitancy) in a field experiment. In doing so, our study sheds light on the importance of host-parasite interactions in shaping life histories and behavior in placental live-bearing fish.

\subsection{Material and methods}

\subsubsection{Study species and collection sites}

Poeciliopsis retropinna, a live-bearing fish species in the family Poeciliidae, reaches a maximum standard length of approximately $80 \mathrm{~mm}$. This species is found in freshwater streams of varying water velocity in Costa Rica and Panama (Bussing, 2002). During gestation, $P$. retropinna females transfer nutrients to developing embryos via a placenta (Pollux et al., 2009). The degree of post-fertilization maternal provisioning in this species is extensive, with offspring increasing in dry mass more than 100-fold during gestation $(\mathrm{MI}=117)$ (Reznick et al., 2002). Moreover, P. retropinna has superfetation, the ability to carry several broods at different developmental stages (Hagmayer et al., 2020c).

During February and March 2017 and 2018, P. retropinna were collected at 19 different locations in the Rio Terraba and Rio Coto drainages in the province of Puntarenas, Costa Rica (Table S.5.1). Each location was characterized by measuring (i) elevation above sea level, (ii) mean river width, (iii) mean river depth, and (iv) mean water velocity (Supplementary Methods S.5.1.1). At each location, 5-37 adult females were collected using seine and cast nets, euthanized with an overdose of MS-222, and preserved in $5 \%$ formaldehyde.

\subsubsection{Laboratory measurements}

Maternal standard length and the proportion of body fat were measured using established protocols (Supplementary Methods S.5.1.2). The intensity of parasite infestation was quantified by counting the number of black spots on each preserved female. The ovaries were subsequently dissected to count the total number of embryos (i.e. fecundity), regressors (i.e. aborted embryos), broods at different developmental stages (i.e. superfetation), embryos in a given brood (i.e. brood size), and to determine the developmental stage and average dry mass of the embryos in a brood (Table 5.1). The developmental stages are based on morphological criteria described in Haynes (1995) and range from 0 (eggs at fertilization, no development) to 45 (fully developed embryos). Fecundity was calculated by excluding stage 0 eggs, since it was difficult to assess if they were fertilized. 
Instead, to ensure that all eggs in our study were fertilized, embryos at developmental stage 2 , rather than 0 , were defined as 'eggs at fertilization'.

Table 5.1. Summary of maternal life-history traits.

\begin{tabular}{ll}
\hline & \multicolumn{1}{c}{ Maternal life-history traits } \\
\hline Egg mass at fertilization & Dry mass of eggs at fertilization (i.e. developmental stage 2) \\
Offspring mass at birth & Dry mass of fully-developed embryos (i.e. developmental stage 45) \\
Proportion egg fat & Egg fat at fertilization divided by dry mass of eggs at fertilization \\
Proportion offspring fat & Offspring fat at birth divided by offspring dry mass at birth \\
Absolute reproductive & Total dry mass allocated to reproduction (i.e. embryo dry mass, regres- \\
allotment & sor dry mass, and placental dry mass) \\
Average brood size & Average number of embryos in a brood for a given mother \\
Fecundity & Number of embryos carried by a female counted across all broods ex- \\
& cluding stage 0 embryos \\
Superfetation & Number of broods at different developmental stages \\
Abortion incidence & Number of regressors (i.e. aborted embryos) divided by the sum of the \\
& number of regressors and embryos \\
\hline
\end{tabular}

\subsubsection{Behavioral trials}

Boldness was assessed on the 27th and 28th of February 2020 between 11:00 and 16:00 in a single population of Poeciliopsis retropinna from Rio Tinoco, Costa Rica. In the morning of each day, 32-35 P. retropinna were collected from closely located pools using a seine net and stored in a 120-L plastic bucket covered with a lid. The sample consisted of adults, defined as large (potentially pregnant) females and mature males (fully developed gonopodium present); immatures, defined as small (non-pregnant) females and males that did not have fully developed gonopodia; and juveniles, defined as fish $<2 \mathrm{~cm}$. Pregnancy in this species is indicated by a dark gravid spot in the belly area. For the statistical analysis, however, we only used individuals that could be sexed (i.e. 40 adults and 21 immatures). To prevent the same individuals from being caught on different days, the same pools were not sampled twice. Prior to the behavioral trial, an individual was randomly selected from the bucket and placed into a glass container $(30 \times 20 \times 2 \mathrm{~cm})$ that minimizes bending movement of the fish. The fish was photographed from both sides and the top to (i) determine sex, (ii) quantify parasite load, and (iii) measure standard length using the image analysis software Image J (Abràmoff et al., 2004).

The experimental set-up to quantify boldness consisted of a dark plastic box $(17 \times 12 \times 11.5$ $\mathrm{cm})$ with a non-transparent lid placed on the top and a trapdoor $(7.5 \mathrm{~cm}$ wide and 9.5 cm high) in the front (Brown et al., 2005). A metal ring was placed underneath the box to form a $\mathrm{D}$ in a radius of $8 \mathrm{~cm}$ in front of the box (Fig. S.5.1). The box was positioned in approximately $15 \mathrm{~cm}$ of water at the edge of the pool from which the fish were collected on day 1. As a result, all fish were provided with the same experimental conditions, except 
that the fish collected at day 1 were released into an environment with which they were more familiar than the fish collected on day 2. We statistically accounted for variation in behavior between different days. Each fish was gently poured into the dark plastic box and allowed to acclimate for 2 min. A single fish was measured at a time. After the acclimation period, the trapdoor in the front of the box was opened and the fish was free to emerge and to swim into the pool. For each fish, both the time taken to emerge from the box after opening the trapdoor and the time to cross the metal ring was recorded. If the fish had not emerged from the box and crossed the metal ring after $10 \mathrm{~min}$, we terminated the trial. Boldness was then defined as (i) the time taken for the fish's snout to emerge from the box (boldness score), and (ii) the time the fish took to cross the metal ring minus the time it took to emerge from the box (hesitancy) (Brown et al., 2005).

\subsubsection{Statistical analysis}

All analyses were carried out in R v 3.6.3 (R Core Team, 2020): mixed models were fitted in a Bayesian framework using the MCMCglmm package (Hadfield, 2010). Convergence was assessed by visual examination of the traces and the autocorrelations of the parameter chain was checked to be less than 0.1. The priors, number of MCMC chains, iterations, burnin, and thinning are given in the Supplementary Information. Negative binomial models were fitted using the glmmTMB package (Brooks et al., 2017).

To identify site-specific environmental sources of variation in black spot infestation, we modeled the proportion of parasitized individuals per sampling location in a series of generalized linear models using Maximum Likelihood and a logit link for the binomialdistributed response (Table S.5.2). Fixed effects in the full model included the elevation of the sampling location, mean river width and depth, as well as mean water velocity (all z-standardized). The models were ranked on the basis of Akaike's information criterion adjusted for small sample sizes (AICc) (Burnham \& Anderson, 2002). However, collinearity among the environmental variables (Table S.5.3) causes difficulties in choosing the 'best' model, as several models each containing different (but correlated) predictors may provide similar fits (Freckleton, 2011). Rather than selecting the 'best' model according to the AICc, we computed model-averaged parameters based on the models with $\triangle \mathrm{AICc}<2$ (Grueber et al., 2011). Specifically, each parameter estimate was averaged over the models in which that predictor appears and was weighted by the summed weights of these models (Burnham \& Anderson, 2002). For the subsequent statistical analyses, we only used the data from locations where black spot disease (BSD) was found (at 16 out of 19 sampling locations; Table S.5.1).

To quantify individual sources of variation in black spot infestation, we fitted parasite load (number of black spots per female) as a function of the proportion of maternal body fat and standard length in a generalized linear mixed model using Maximum Likelihood and a log link for the negative binomial-distributed response. The negative binomial model accounts for over-dispersion arising from individual heterogeneity in parasite load. 
Sampling location (i.e. population) was fitted as random intercept accounting for spatial non-independence of observations.

The potential life-history consequences of black spot infestation were evaluated by fitting the maternal life-history traits (egg mass at fertilization, offspring mass at birth, proportion of egg and offspring fat, reproductive allotment, average brood size, fecundity, superfetation, and abortion incidence) as a function of parasite load (number of black spots per female) in a multivariate (generalized) linear mixed effects model allowing for the covariance between the residuals of all responses. Additional fixed effects included the proportion of maternal body fat, which is believed to be a good indicator of fish condition (Leips et al., 2013), and maternal standard length (Hagmayer et al., 2018). In the case of reproductive allotment, fecundity and superfetation, the developmental stage of the most-developed brood was fitted as an additional fixed effect to account for females early in the reproductive cycle. Sampling location (i.e. population) was fitted as random intercept accounting for spatial non-independence of observations.

To optimize normality and homoscedasticity of model residuals, reproductive allotment was ln-transformed, and abortion incidence, proportion of egg and offspring fat, and maternal body fat were arcsin square-root transformed. Fecundity and superfetation were fitted in generalized linear mixed effects models using a log link for the Poisson-distributed responses.

Behavioral responses to black spot infestation were evaluated by first modelling the probability of a fish emerging from the box and crossing the metal ring. For this, a binary variable indicting whether an individual emerged from the box and crossed the metal ring was fitted as a function of parasite load (number of black spots), sex, day, and standard length in a generalized linear model using Maximum Likelihood and a logit link for the binomial-distributed response. An additional predictor was the time difference ( $\mathrm{s}$ ) between the behavioural trial and capture ( $z$-standardized). However, this effect was not significant $\left(\chi_{12}=0.560, P=0.454\right)$ and thus excluded from any further analysis. Second, both the boldness score (the time taken to emerge from the box) and hesitancy (the time the fish took to cross the metal ring minus the time it took to emerge from the box) were each fitted as a function of parasite load (number of black spots), sex, day, and standard length in generalized linear models using Maximum Likelihood and a log link for the quasipoisson-distributed responses. In both cases, the time difference (s) between the behavioural trial and capture ( $z$-standardized) was not significant (boldness score: $\mathrm{F}_{1}=0.788, P=0.379$; hesitancy: $\left.\mathrm{F}_{1}=0.035, P=0.853\right)$ and thus excluded from any further analysis.

To compare the strength of individual relationships, all regression coefficients $(\beta)$ were standardized by multiplying with the phenotypic standard deviation of the predictor variable and dividing by the phenotypic standard deviation of the response variable (Schielzeth, 2010). In the case of non-Gaussian distributed responses, the phenotypic standard deviation of the response variable was indirectly estimated according to Menard (2011). The resulting effect sizes $\left(\beta^{*}\right)$ take values between -1 and 1 . 


\subsection{Results}

\subsubsection{Environmental sources of variation in black spot infestation among populations}

We found substantial variation in black spot infestation among and within the populations of Poeciliopsis retropinna (Fig. 5.1). Particularly, the proportion of parasitized individuals was negatively correlated with elevation of the sampling location $\left(\beta^{*}=-0.317, z=2.068\right.$, $P=0.039$; Table S.5.4; Fig. 5.2a), mean river width $\left(\beta^{*}=-0.378, z=2.475, P=0.013\right.$; Table S.5.4; Fig. 5.2a), and mean river depth $\left(\beta^{*}=-0.337, z=2.351, P=0.019\right.$; Table S.5.4; Fig. 5.2a). Moreover, the proportion of parasitized individuals tended to negatively correlate with mean water velocity $\left(\beta^{*}=-0.250, z=1.580, P=0.114\right.$; Table S.5.4; Fig. $5.2 \mathrm{a})$.

(a)
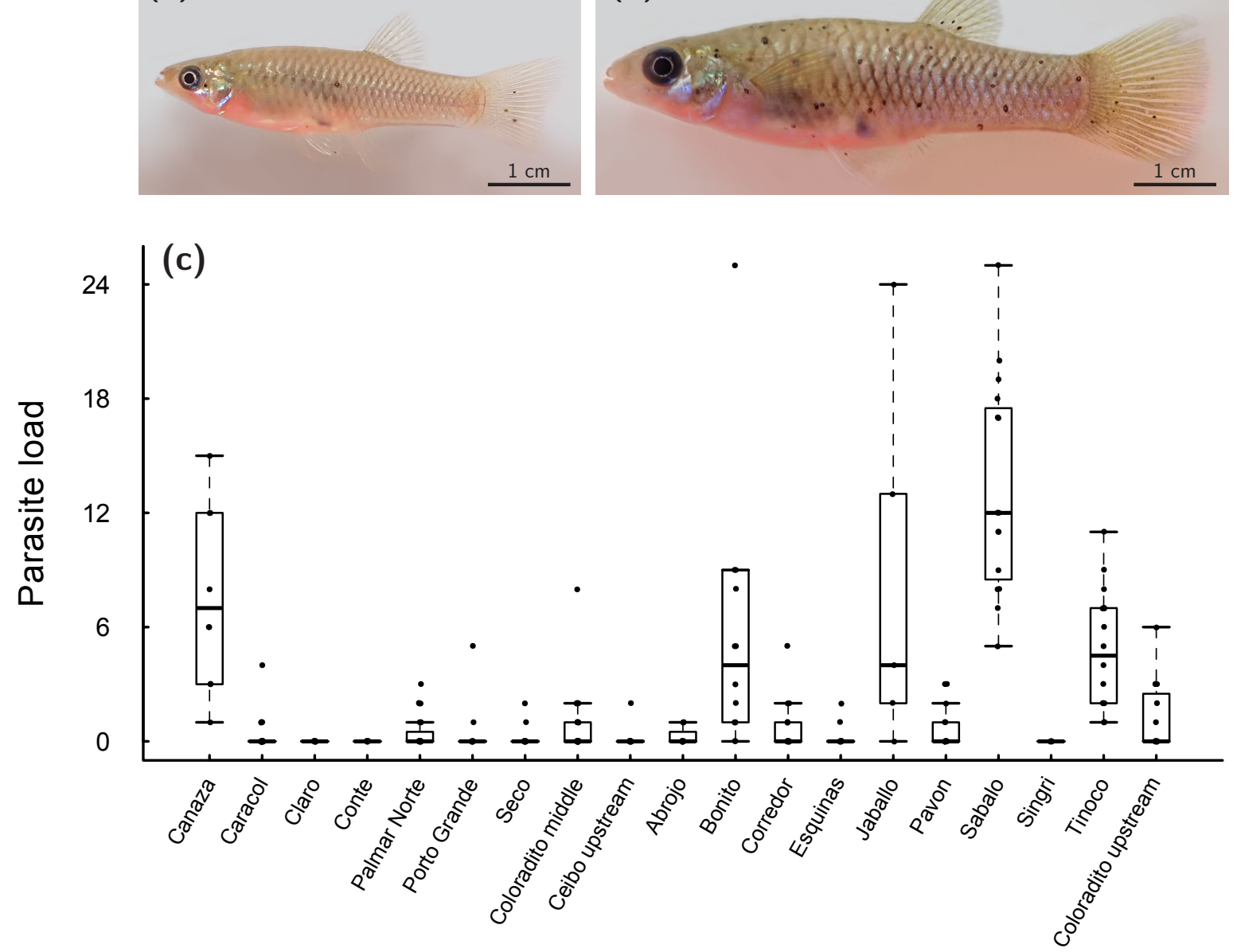

(b)

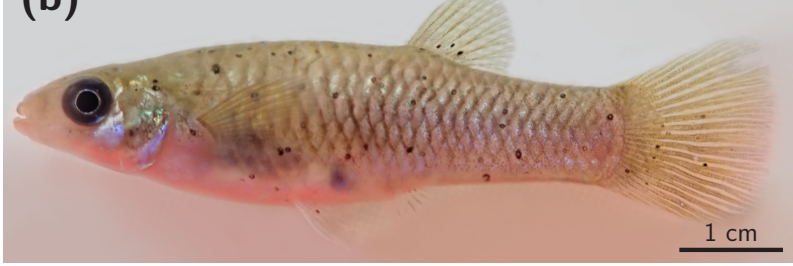

Fig. 5.1. (a) Immature and (b) adult female Poeciliopis retropinna with low and high parasite load (number of black spots), respectively. (c) Boxplot showing variation in parasite load among and within sampling locations (i.e. populations). 


\subsubsection{Maternal sources of variation in black spot infestation within populations}

Individual parasite load (number of black spots per female) was positively associated with maternal standard length $\left(\beta^{*}=0.302, z=3.708, P<0.001\right.$; Table S.5.5; Fig. $5.2 \mathrm{~b})$ and tended to decrease with increasing proportion of maternal body fat, though not significantly $\left(\beta^{*}=-0.186, z=-1.709, P=0.087\right.$; Table S.5.5; Fig. 5.2c).
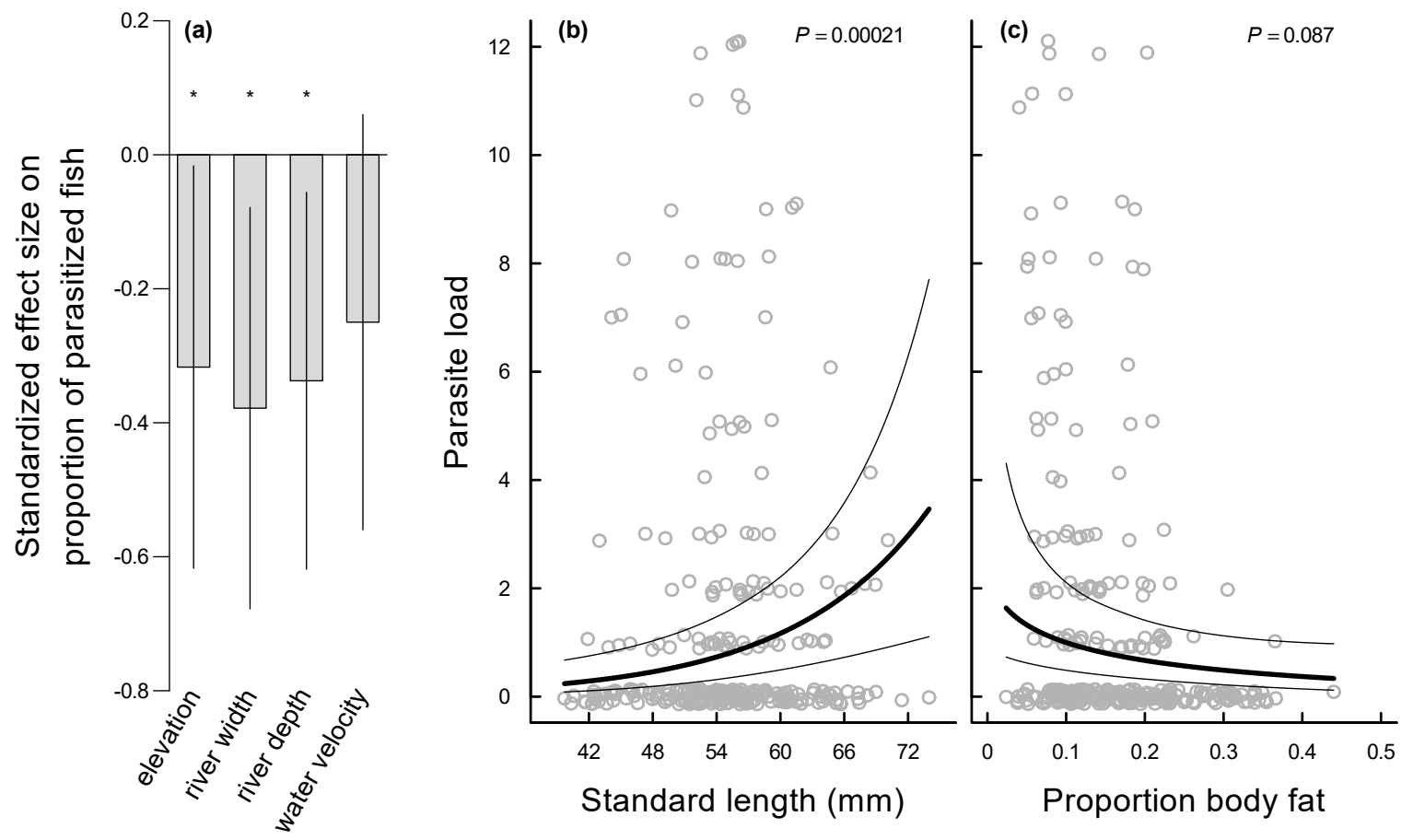

Fig. 5.2. (a) Standardized effect size $( \pm 95 \% \mathrm{Cl})$ of the environmental variables (elevation of the sampling location, mean river width, mean river depth, mean water velocity) on the proportion of parasitized Poeciliopsis retropinna females within a sampling population. The effect sizes are calculated based on the model-averaged parameters given in Table S.5.4 and take values between -1 and 1. Significant relationships are indicated with an asterisk $\left(^{*}\right)$. (b) Individual parasite load (number of black spots per female) as a function of maternal standard length $(n=302)$. (c) Individual parasite load as a function of proportion of maternal body fat $(n=302)$. The relationships in (b) and (c) were estimated in the generalized linear mixed effects model described in Table S.5.5. The model predictions account for the proportion of maternal body fat in (b) and maternal standard length in (c) that are kept constant at the overall mean (i.e. body fat $=0.15$, standard length $=55 \mathrm{~mm}$ ). Data points correspond to the 'jittered' raw data. $P$-value is given at the top. 


$\begin{array}{lllll}- \text { Canaza } & \diamond \text { Palmar Norte } & \diamond \text { Ceibo upstream } & \otimes \text { Esquinas } & \Delta \text { Singri } \\ \triangle \text { Caracol } & \nabla \text { Porto Grande } & \oplus \text { Abrojo } & \square \text { Jaballo } & \text { Tinoco } \\ + \text { Claro } & \otimes \text { Seco } & \otimes \text { Bonito } & \square \text { Pavon } & \bullet \text { Coloradito upstream } \\ \times \text { Conte } & * \text { Coloradito middle } & \oplus \text { Corredor } & \bullet \text { Sabalo }\end{array}$
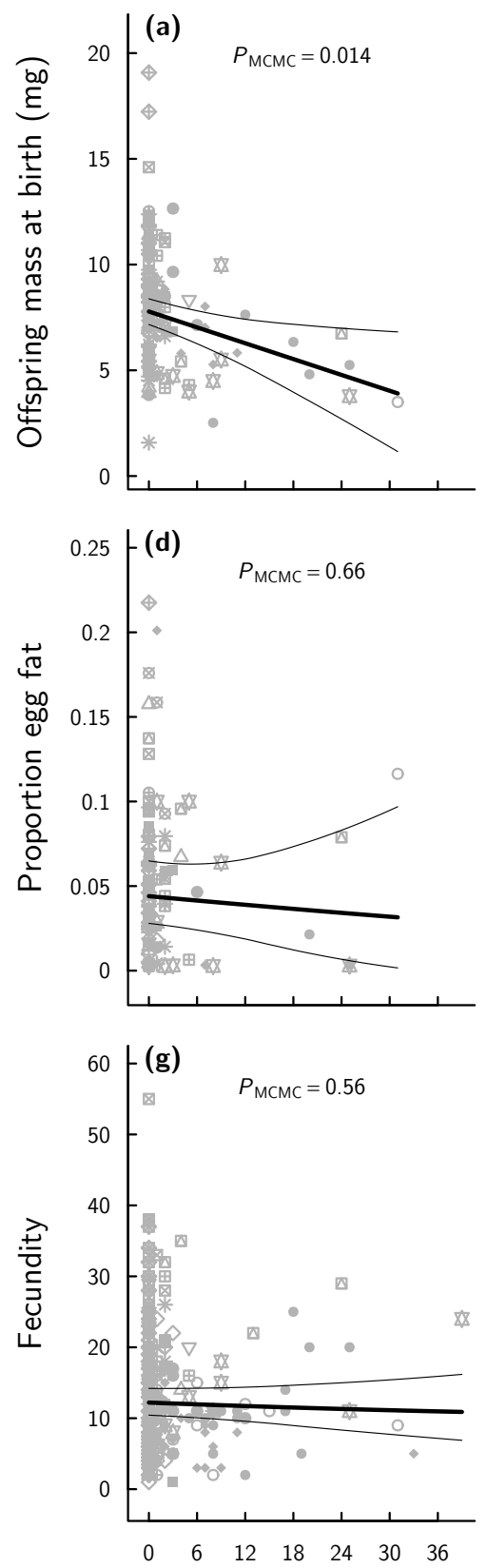
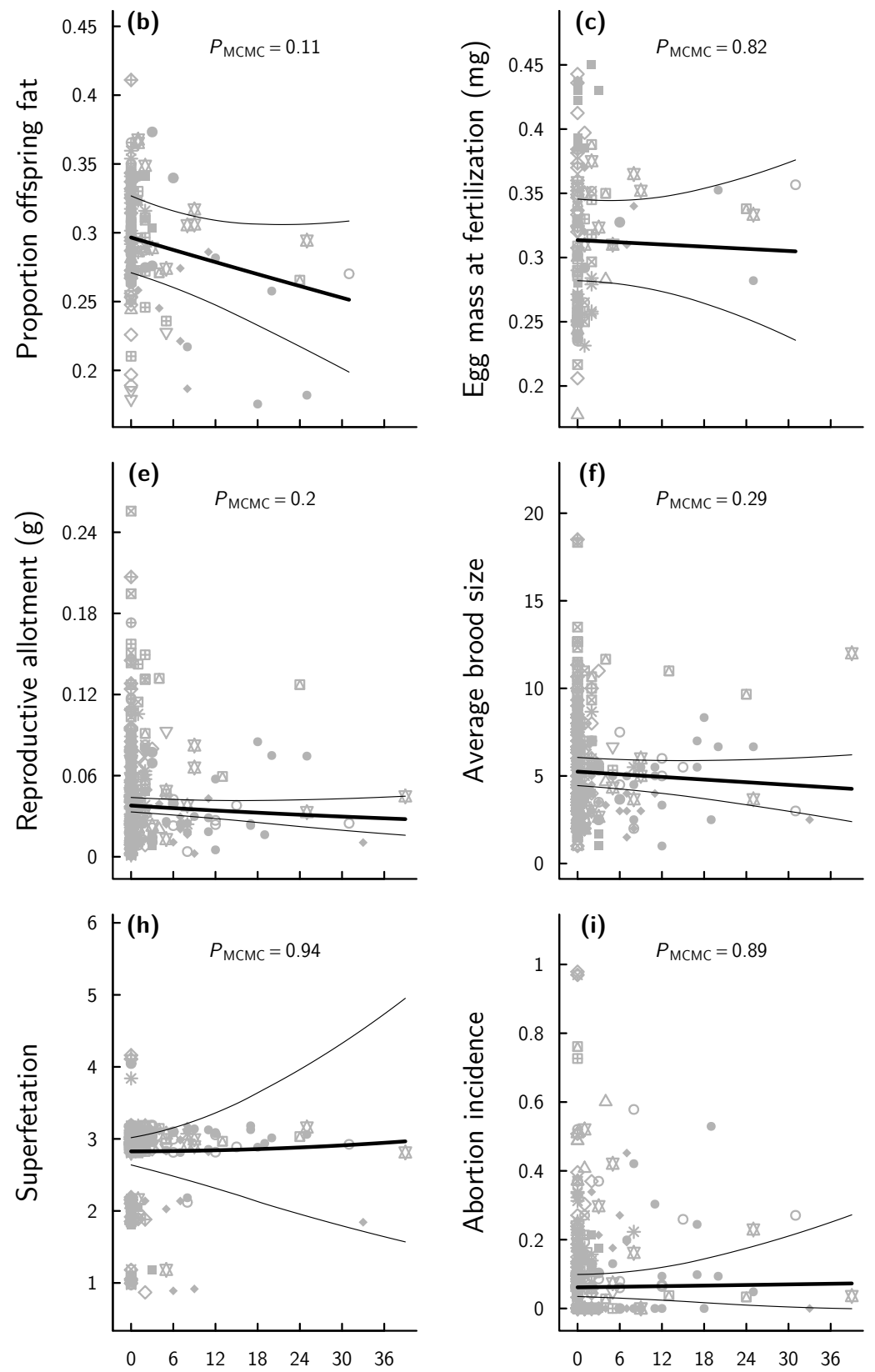

Parasite load 
Fig. 5.3. Life-history characteristics of Poeciliopsis retropinna in relation to black spot infestation. (a) Offspring dry mass at birth (developmental stage 45) $(n=132)$, (b) proportion offspring fat at birth $(n=132)$, (c) egg dry mass at fertilization (developmental stage 2$)(n=109)$, (d) proportion egg fat at fertilization $(n=99),(\mathbf{e})$ absolute dry reproductive allotment $(n=287)$, (f) average brood size ( $n=282),(g)$ maternal fecundity (number embryos in all broods combined) $(n=282)$, (h) degree of superfetation $(n=288)$, and (i) abortion incidence $(n=288)( \pm 95 \%$ $\mathrm{Cl}$ ) as a function of parasite load (number of black spots per female) estimated in the models described in Table S.5.6. All model predictions account for the proportion of maternal body fat and maternal standard length, which are kept constant at the overall population mean (i.e. body fat $=0.15$, standard length $=55 \mathrm{~mm})$. In (e) and $(\mathbf{g})-(\mathbf{h})$, the developmental stage of the mostdeveloped brood carried by the female is kept constant at the overall median (i.e. developmental stage 42.5). Data points correspond to the population-specific raw data. $P_{\mathrm{MCMC}}$-value is given at the top of each panel.

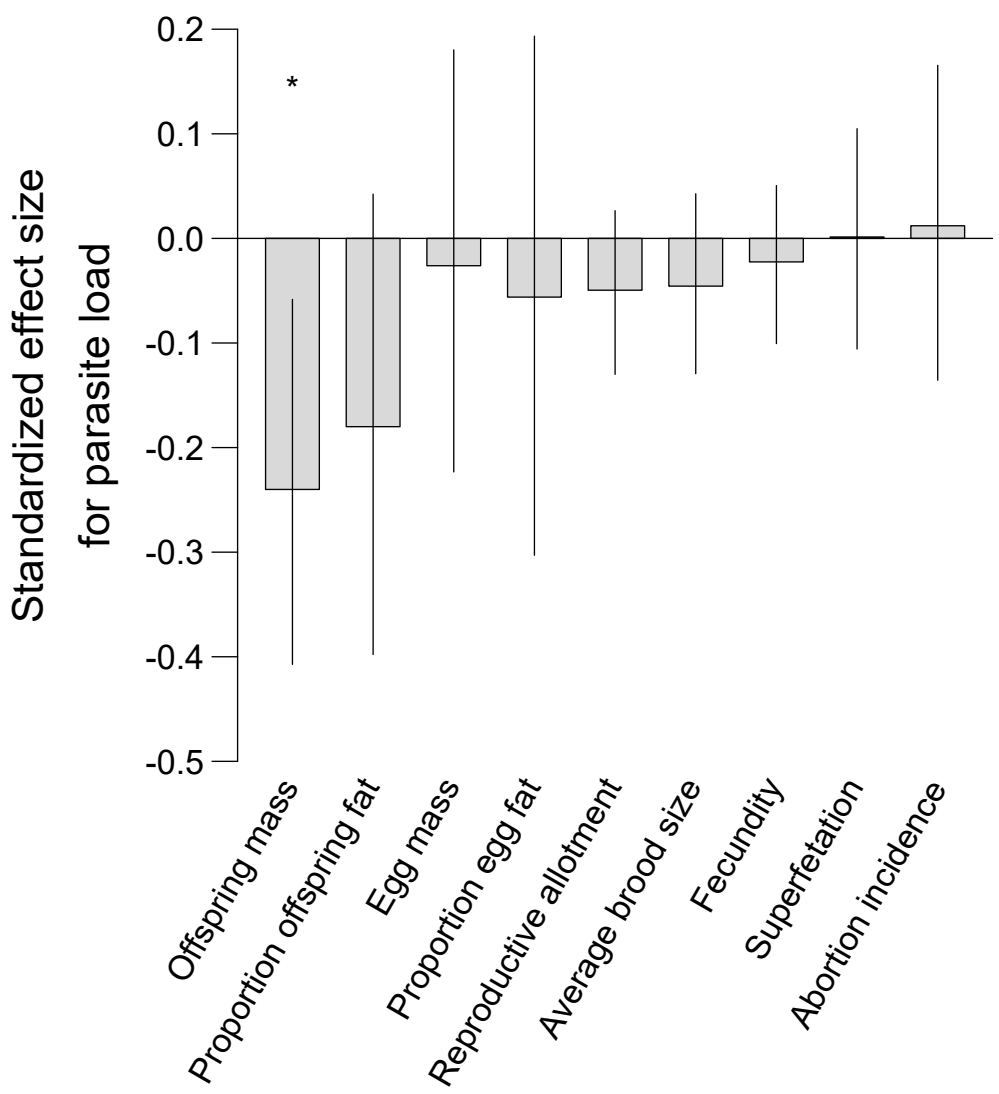

Fig. 5.4. Standardized effect size $( \pm 95 \% \mathrm{Cl})$ of parasite load (number of black spots per female) on maternal life-history traits (offspring dry mass at birth, proportion offspring fat at birth, egg dry mass at fertilization, proportion egg fat at fertilization, absolute dry reproductive allotment, average brood size, fecundity, superfetation, abortion incidence). The effect sizes take values between -1 and 1 . Significant relationships are indicated with an asterisk $\left(^{*}\right)$. 


\subsubsection{Life-history consequences of black spot infestation}

Independent of maternal standard length and body fat, heavily parasitized female $P$. retropinna produced smaller offspring at birth $\left(\beta_{\text {post.mean }}^{*}=-0.240,95 \% \mathrm{CI}=-0.407--\right.$ 0.058, $P_{\mathrm{MCMC}}=0.014$; Table S.5.6a; Fig. 5.3a, 5.4) that tended to have a lower proportion of body fat $\left(\beta_{\text {post.mean }}^{*}=-0.180,95 \% \mathrm{CI}=-0.398-0.042, P_{\mathrm{MCMC}}=0.110\right.$; Table S.5.6b; Fig. 5.3b, 5.4). In contrast, parasite load did not correlate with egg mass $\left(\beta_{\text {post.mean }}^{*}=-0.026,95 \% \mathrm{CI}=-0.223-0.180, P_{\mathrm{MCMC}}=0.818\right.$; Table S.5.6c; Fig. 5.3c, 5.4) or the proportion of egg fat at fertilization $\left(\beta_{\text {post.mean }}^{*}=-0.056,95 \%\right.$ $\mathrm{CI}=-0.303-0.193, P_{\mathrm{MCMC}}=0.658$; Table S.5.6d; Fig. 5.3d, 5.4). Likewise, parasite load was not associated with absolute dry reproductive allotment $\left(\beta_{\text {post.mean }}^{*}=-0.050\right.$, 95\% CI $=-0.130-0.027, P_{\mathrm{MCMC}}=0.198$; Table S.5.6e; Fig. 5.3e, 5.4), average brood size $\left(\beta_{\text {post.mean }}^{*}=-0.046,95 \% \mathrm{CI}=-0.129-0.043, P_{\mathrm{MCMC}}=0.288\right.$; Table S.5.6f; Fig. $5.3 f, 5.4)$, fecundity $\left(\beta_{\text {post.mean }}^{*}=-0.022,95 \% \mathrm{CI}=-0.101-0.051, P_{\mathrm{MCMC}}=0.560\right.$; Table S.5.6g; Fig. 5.3g, 5.4), superfetation $\left(\beta_{\text {post.mean }}^{*}=0.001,95 \%\right.$ CI $=-0.106-0.105$, $P_{\mathrm{MCMC}}=0.936$; Table S.5.6h; Fig. $\left.5.3 \mathrm{~h}, 5.4\right)$, or abortion incidence $\left(\beta_{\text {post.mean }}^{*}=0.012\right.$, $95 \% \mathrm{CI}=-0.136-0.165, P_{\mathrm{MCMC}}=0.892$; Table S.5.6i; Fig. 5.3i, 5.4).

\subsubsection{Boldness responses to black spot infestation}

The probability of emerging from the box and crossing the metal ring tended to decrease with increasing standard length, though not significantly $\left(\beta^{*}=-0.732, z=-1.715\right.$, $P=0.086$; Table S.5.7; Fig. 5.5a). This decrease was similar in females and males $\left(\beta^{*}=0.228, z=0.663, P=0.507\right.$; Table S.5.7; Fig. 5.5a). In contrast, the probability of emerging from the box and crossing the metal ring did not correlate with parasite load (number of black spots) $\left(\beta^{*}=0.084, z=0.254, P=0.800\right.$; Table S.5.7; Fig. 5.5a), but tended to correlate with measurement day $\left(\beta^{*}=-0.443, z=-1.761, P=0.078\right.$; Table S.5.7).

The boldness score (the time taken to emerge from the box) did not correlate with standard length $\left(\beta^{*}=0.082, t=0.448, P=0.656\right.$; Table S.5.8; Fig. 5.5b), sex $\left(\beta^{*}=0.080\right.$, $t=0.606, P=0.547$; Table S.5.8; Fig. 5.5b), and parasite load $\left(\beta^{*}=-0.070, t=-0.372\right.$, $P=0.712$; Table S.5.8; Fig. 5.5b). However, fish measured on day 2 took longer to emerge from the box than fish measured on day $1\left(\beta^{*}=0.435, t=3.232, P=0.002\right.$; Table S.5.8).

Finally, hesitancy (the time the fish took to cross the metal ring minus the time it took to emerge from the box) tended to increase with increasing standard length, though not significantly $\left(\beta^{*}=0.375, t=1.824, P=0.075\right.$; Table S.5.9; Fig. 5.5c). Moreover, males tended to be more hesitant than females $\left(\beta^{*}=0.249, t=1.853, P=0.071\right.$; Table S.5.9; Fig. 5.5c). In contrast, hesitancy was not associated with parasite load $\left(\beta^{*}=-0.055\right.$, $t=-0.325, P=0.747$; Table S.5.9; Fig. 5.5c) or measurement day $\left(\beta^{*}=0.198, t=1.650\right.$, $P=0.107 ;$ Table S.5.9). 

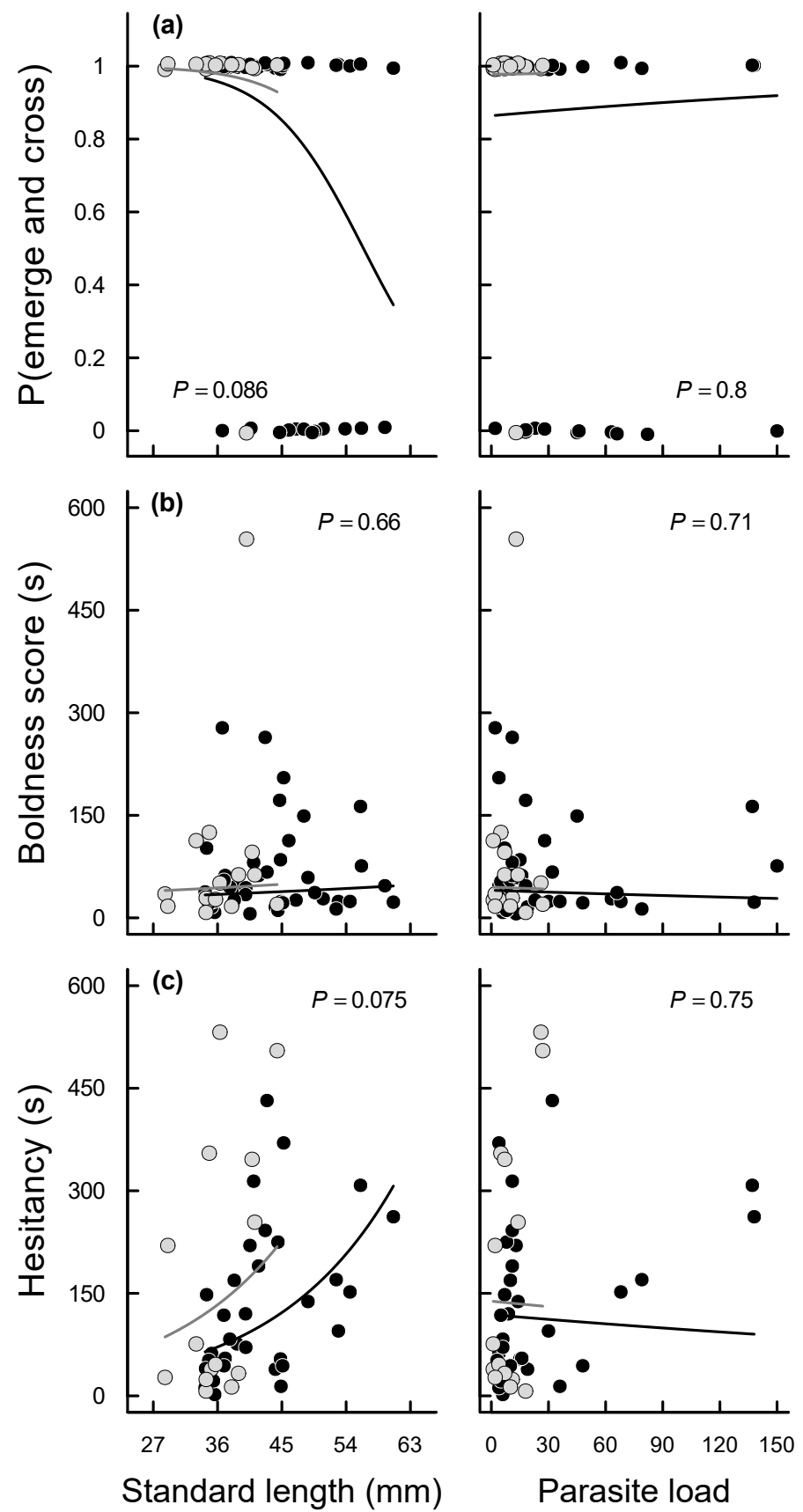

Fig. 5.5. Boldness responses of Poeciliopsis retropinna to black spot infestation assessed in the field. (a) Probability of emerging from the box and crossing the metal ring $(n=60)$, (b) boldness score (time taken to emerge from the box) $(n=56)$, and (c) hesitancy (the time the fish took to cross the metal ring minus the time it took to emerge from the box) ( $n=46)$ as a function of sex (black line: females; grey line: males), standard length (left panels) and parasite load (number of black spots; right panels) estimated in the models described in Table S.5.7-S.5.9. All model predictions account for the number of parasites (left panels) and standard length (right panels), which are kept constant at the sex-specific population mean (i.e. number parasites $_{q}=29$, number

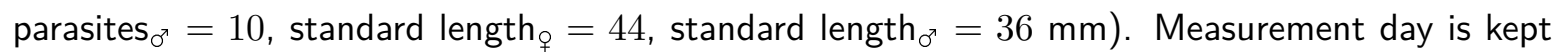
constant at day 1. Data points (black: females; grey: males) correspond to the raw data; 'jittered' in (a). P-value for the effect of standard length (left panels) and parasite load (right panels) are given in each panel. 


\subsection{Discussion}

Parasites can affect the survival and reproductive success of their hosts (Bush et al., 2001). In order to reduce these fitness costs of parasitism, hosts can respond on a physiological, morphological or behavioral level (Richner, 1998). Here we examined the importance of black spot disease (BSD) in shaping life history and behavior in the placental livebearing fish species Poeciliopsis retropinna. We found substantial variation in black spot infestation among and within the study populations. The proportion of parasitized individuals in a population was negatively correlated with elevation of the sampling location, mean river width, mean river depth, and tended to negatively correlate with mean water velocity. Within populations, individual parasite load (i.e. the number of black spots per female) was positively associated with maternal standard length, but tended to decrease with increasing proportion of maternal body fat. Parasite load did not correlate with other maternal life-history traits, such as fecundity, reproductive allotment, superfetation, or abortion incidence. However, heavily parasitized females produced smaller offspring at birth that tended to have a smaller proportion of body fat, while the size and quality of eggs at fertilization remained unaffected. Finally, independently of body size, parasite load was not associated with an individual's boldness (boldness score and hesitancy).

\subsubsection{Environmental sources of variation in black spot infestation among populations}

The trematode that is causing BSD relies on water snails and fish as intermediate, and piscivorous birds as final, hosts during its life-cycle (Lane \& Morris, 2010). Environmental variables are thereby likely to affect the relative abundance of hosts and the probability of free-living parasite life stages successfully invading the hosts. Relating parasitism to environmental variables may, therefore, shed light on the abiotic conditions that favor the chance of the parasite completing its life-cycle.

For example, we found that the proportion of parasitized individuals increases with decreasing elevation of the sampling location. In other words, the probability of BSD is higher in coastal rivers near the ocean compared to high-altitude inland rivers. In Costa Rica, inland rivers are typically enclosed by dense canopy and drain from steep mountain environments, whereas lowland rivers are often free of vegetation. Piscivorous birds are visually oriented predators and shade due to increased vegetation cover decreases their foraging success (Trexler et al., 1994; Penaluna et al., 2016). Bird predation is thus likely higher for fish in the open canopy lowland rivers compared to the closed canopy inland rivers. Because piscivorous birds are obligatory final hosts, open canopy areas may increase the chance of the parasite completing its life-cycle, thereby partly explaining the higher proportion of infested females in lowland regions. A decrease in elevation is furthermore associated with an increase in water temperature. The production of cercariae in intermediate snail hosts has been shown to be profoundly influenced by increasing 
temperature, which causes an increase in cercarial output and hence a greater number of cercarial infective stages in aquatic habitats (Poulin, 2006). Thus, the increasing proportion of infected individuals with decreasing elevation may be the result of both warmer water and open canopies, which facilitate parasite reproduction in their intermediate and final hosts, respectively.

We further found that the proportion of parasitized individuals decreases with increasing river width and depth. In other words, the probability of BSD is higher in smaller (i.e. narrower and shallower) compared to larger (i.e. wider and deeper) rivers. The density of the parasite's intermediate hosts (i.e. the number of water snails and fish per unit volume of water) is likely to be inversely related to river size. As a result, the chance of the parasite finding a host may decrease with increasing river size. Moreover, piscivorous birds are more effective predators in shallow near-shore water (Whitfield \& Cyrus, 1978; Kramer et al., 1983). This means that the higher observed proportion of infested individuals with decreasing river width and depth might be related to both a higher parasite density and higher probability of parasitized fish getting eaten by their final hosts thereby completing the parasite life-cycle.

The probability of BSD further tended to decrease with increasing water velocity, being higher in slow flowing or stagnant rivers compared to fast flowing rivers. This may be related to the locomotor ability of the miracidia and cercariae that infect water snails and fish, respectively. Both of these life stages are relatively small $(<1 \mathrm{~mm})$ and although they are motile free-living stages (Hoffman \& Putz, 1965), they have very limited swimming capabilities (Koehler et al., 2012). The chance that free-swimming miracidia and cercariae find and successfully invade intermediate snail or fish hosts might therefore decrease with increasing flow velocities, because they may get washed away before successfully invading a host (Marcogliese, 2016).

Nevertheless, the observed correlations with parasite load should be interpreted with care, as we only measured a limited set of environmental variables. The exclusion of variables that are potentially important for parasite ecology could lead to spurious correlations. Therefore, future studies focusing on parasite ecology should include a broader set of local environmental variables (e.g. density of snails and piscivorous birds).

\subsubsection{Maternal sources of variation in black spot infestation within populations}

We found that individual parasite load (number of black spots per female) is positively correlated with maternal standard length: larger fish carry more parasites. This is likely due to two non-mutually exclusive effects: a time (age) effect and a fish size effect. First, the fibrous capsule and melanocyst wall surrounding the parasite prevent parasites from easily being exocytosed from the host (Cureton et al., 2011). This means that parasites will remain embedded in the skin until the fish dies (or gets eaten by the parasite's final host) and that the number of parasites on an individual will (independent of fish size) increase over time due to continued exposure to the parasites. Second, larger fish 
are simply able to carry more parasites, because growth is associated with an increase in skin surface area to which parasites can attach. Therefore, the observed relationship between parasite infestation and standard length may be the result of (i) an accumulation of parasite infection over time (with age), and (ii) increase in skin surface area to which parasites can attach during body growth.

Independent of standard length, parasite load tended to be higher for females with low proportion of body fat, though not significantly. The proportion of maternal body fat is believed to be a good indicator of fish condition (Leips et al., 2013) and may thus affect, or be affected by, parasitism. Specifically, a poor body condition might be expected to be associated with a high parasite load, either because worse-conditioned individuals are more susceptible to the parasite (less energy for immune function), or the host's response to the parasite encapsulation is energetically expensive (Lane \& Morris, 2010; Cureton et al., 2011). For instance, BSD was shown to reduce the body condition of smallmouth bass (Micropterus dolomieui) (Hunter \& Hunter, 1938) and juvenile bluegill sunfish (Lepomis macrochiris) (Lemly \& Esch, 1984). However, there was no evidence for a reduced body condition in infected females of the poeciliid fish Gambusia affinis (Cureton et al., 2011). Likewise, there was no clear association of parasite load with body condition in our study, and thus, BSD may not invoke an energetic demand strong enough to reduce maternal fat reserves in P. retropinna.

\subsubsection{Life-history consequences of black spot infestation}

Parasites can change the allocation of resources to different functions such as growth, reproduction, survival, and maintenance by inducing shifts in the optimum of life-history traits (Michalakis \& Hochberg, 1994; Sheldon \& Verhulst, 1996; Richner, 1998).

We found that BSD does not correlate with maternal fecundity, reproductive allotment, superfetation, or abortion incidence. However, independent of maternal body fat and size, heavily parasitized females produce smaller and worse-conditioned offspring at birth, while egg size and quality at fertilization remain unaffected. Specifically, an increase in maternal parasite load by five black spots, decreases the mass of offspring at birth by $8.73 \%$ and the proportion of offspring body fat by $2.57 \%$. In placental live-bearing fish, resource allocation to offspring takes place during two distinct periods (Wourms, 1981). Part of the nutrients are supplied pre-fertilization during oogenesis and stored as high-energy yolk (Wallace \& Selman, 1981). The rest of the nutrients are transferred post-fertilization to the developing embryos throughout gestation (Wourms, 1981). The latter is achieved through a follicular placenta, roughly an analog to the mammalian placenta (Turner, 1940). In general, the amount of resources a female can transfer to her developing offspring per unit of time is the result of a balance between maternal energy uptake (via feeding), her own caloric utilization (maintenance) and the amount of excess energy that is subsequently available for reproduction (Stearns, 1992). In parasitic environments, hosts may allocate an increased amount of resources to parasite defense 
that might otherwise have been used for different functions (Sheldon \& Verhulst, 1996). It is thus possible that parasitized females simply have less energy available that can be used to invest in developing embryos, which results in the production of smaller and worse-conditioned offspring at birth.

Furthermore, the degree of post-fertilization maternal provisioning has been shown to be positively correlated with the elaboration of the structures that form the interface between the mother and offspring, and hence to reflect the complexity of placental morphology (Kwan et al., 2015; Olivera-Tlahuel et al., 2019). Producing such a highly elaborate tissue is presumably energetically costly. In mice, maternal malnutrition was shown to impact placental morphology, which consequently leads to impaired placental functioning and insufficient nutrient provisioning to embryos (Connor et al., 2020). In placental poeciliid fish, maternal malnutrition is known to lead to smaller offspring at birth (Reznick et al., 1996b; Banet et al., 2010; Pollux \& Reznick, 2011). Smaller offspring have lower competitive abilities (Bashey, 2006) and lower swimming capabilities (e.g. Dial et al. 2016; Lankheet et al. 2016), and thus, presumably lower fitness in adverse resource environments (Reznick et al., 1996b; Banet et al., 2010; Pollux \& Reznick, 2011). However, to what extent parasitism might potentially negatively impact placental functioning in poeciliid fish, either directly or indirectly by shifting the energy investment required to form an elaborate placental structure more towards other functions, is currently unknown and requires further investigation.

\subsubsection{Boldness responses to black spot infestation}

Parasitism plays a potentially important role in shaping aspects of animal personalities, such as exploration, activity or boldness (Barber \& Dingemanse, 2010). We found that parasite load does not correlate with our measurement of boldness (boldness score and hesitancy) in P. retropinna, suggesting that potential parasite-induced behavioral modifications are not reflected by an individual's boldness in this species. Bolder individuals typically engage in more risky behavior to obtain food (Chapman et al., 2010). This increases resource-intake rates, but also their vulnerability to predators (Godin \& Smith, 1988). The adaptive significance of risk taking, therefore, depends on the cost/benefit ratio of being bold in different situations (Sih et al., 2004). Specifically, parasitism may favor high levels of boldness, when the compensation of the parasite-induced fitness costs is efficient (Kortet et al., 2010). For instance, increased boldness may enable parasitized individuals to effectively compensate for the extra nutritional requirements caused by the parasite (Giles, 1983). Alternatively, parasitism may favor low levels of boldness, when individuals have little or no means of compensating for the costs (Kortet et al., 2010). In P. retropinna, therefore, BSD may not invoke fitness costs large enough to favor either increased or decreased levels of boldness. This is in line with our findings showing that BSD is only weakly associated with a reduced maternal body condition (body fat reserves) or fecundity. 
In contrast, we found that the probability of emerging from the box and crossing the metal ring tends to decrease with increasing standard length in males and females. Moreover, if an individual emerges from the box and crosses the metal ring, larger fish take longer to do so. In other words, larger fish tend to be more hesitant. However, although the effect sizes for both relationships were moderate to large $\left(\beta_{\text {probability }}^{*}=-0.732 ; \beta_{\text {hesitancy }}^{*}=0.375\right)$ neither were significant, which is likely the result of the small sample size and hence low statistical power. The negative relationship between boldness and body size was best explained by the so-called metabolic hypothesis in other poeciliids (Brown \& Braithwaite, 2004; Brown et al., 2005). Specifically, smaller individuals have lower body fat reserves and faster metabolic rates, and may thus be compelled to leave a secure shelter sooner to maximize feeding opportunities (Krause et al., 1998; Skalski \& Gilliam, 2002).

\subsection{Conclusion}

Parasites can change the allocation of resources to different functions such as growth, reproduction, survival, and maintenance by inducing shifts in the optimum of life-history traits (Michalakis \& Hochberg, 1994; Sheldon \& Verhulst, 1996; Richner, 1998). Placental live-bearing fish transfer nutrients to their developing embryos throughout pregnancy via a placenta (Wourms, 1981). Parasite infestation of females during pregnancy could potentially influence embryo development at different gestational stages. We show that BSD is associated with a reduction in post-fertilization maternal provisioning resulting in the production of smaller and worse-conditioned offspring at birth. These parasitized females may be physiologically unable to produce larger offspring, either because of less resources available to invest in developing embryos or because of placental malfunctioning. Our study herewith identified an important potential cost of trematode infestation in placental live-bearing fish, which is a reduced maternal nutrient provisioning to embryos, resulting in smaller size and quality, and potentially lower fitness, of offspring at birth.

\section{S.5 Supplementary Information}

\section{S.5.1 Supplementary Methods}

\section{S.5.1.1 Measurements of water parameters}

The water velocity was measured at each location to the nearest $0.01 \mathrm{~m} \cdot \mathrm{s}^{-1}$ with a Höntzsch Vane Wheel FA current meter (type ZS30 GFE md20 T/100-2/p10, Höntzsch Instruments, Waiblingen, Germany). Depending on the uniformity of the flow, the water velocity was taken at 9-17 incremental observation points across a transect of the stream. At each observation point, the mean water velocity was defined as the average of three repeated measurements at a height above the stream bed equal to 0.4 times the depth 
at that location. When the water depth exceeded $60 \mathrm{~cm}$, the mean water velocity was calculated as the average between the velocities measured at 0.2 times the water depth and 0.8 times the water depth (Hauer \& Lamberti, 2007).

\section{S.5.1.2 Laboratory measurements}

The standard length of preserved specimens was measured to the nearest $\mathrm{mm}$ from the tip of the upper jaw to the outer margin of the hypural plate, using a caliper. Female dry mass was measured to the nearest $0.01 \mathrm{mg}$ on a Mettler Toledo XP205 delta range (Mettler Instruments Corp., Hightstown, New Jersey, USA) after removing the ovary and air-drying the female overnight at $60^{\circ} \mathrm{C}$ in a drying oven. Female lean mass was measured by extracting the fat twice with anhydrous diethyl ether (Fisher Scientific) to remove triglycerides, and by subsequently air-drying and re-weighting the female (see above). The proportion of maternal body fat was then calculated by subtracting maternal lean mass from maternal dry mass divided by maternal dry mass. The embryo dry mass for a given brood was calculated as the dry mass of the brood, measured to the nearest 0.01 mg after air-drying overnight at $60^{\circ} \mathrm{C}$ (see above), divided by the number of embryos in the brood (Pollux \& Reznick, 2011).

Fig. S.5.1. Schematic illustration of the plastic box $(17 \times 12 \times 11.5 \mathrm{~cm})$ used to assess an individual's boldness in the field. The box was positioned in approximately $15 \mathrm{~cm}$ of water on the edge of the pool. A metal ring was placed underneath the box to form a $D$ in a radius of $8 \mathrm{~cm}$ in front of the box. A single fish was placed at a time in the box with a lid on the top. After an acclimation period of $2 \mathrm{~min}, \mathrm{a}$ trapdoor $(7.5 \mathrm{~cm}$ wide and $9.5 \mathrm{~cm}$ high) in the front of the box was opened and the fish was free to emerge and to swim into the pool. Individual fish were observed from above until they crossed the metal ring.

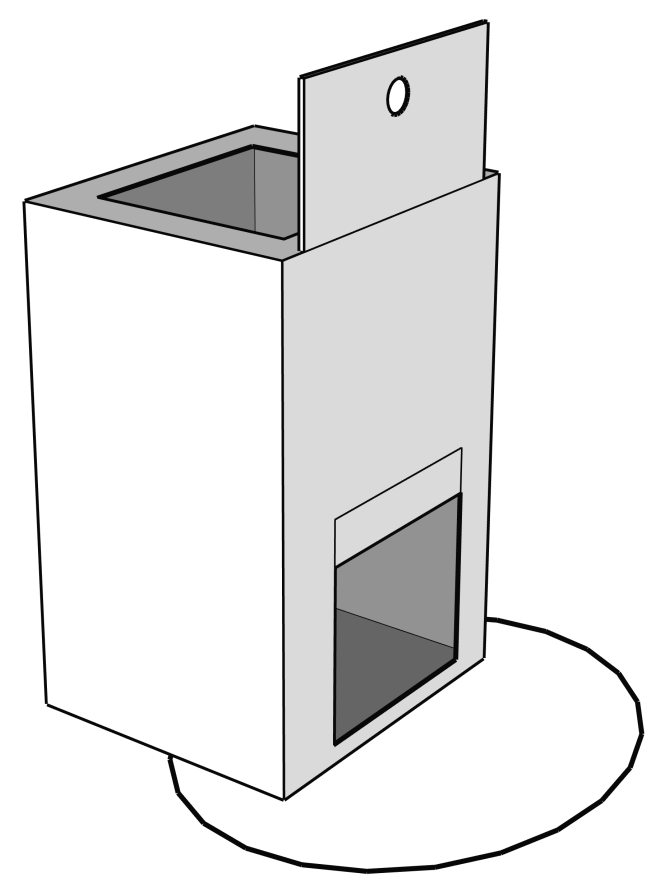


Table S.5.1. Sampling locations (plus coordinates), sampling dates, parasitism (i.e. proportion of parasitized females), and number of collected $(n)$ and pregnant ( $\left.n_{\text {preg }}\right)$ females at each location.

\begin{tabular}{|c|c|c|c|c|c|c|}
\hline Location & River & Parasitism & Coordinates & Sampling date & $n$ & $n_{\text {preg }}$ \\
\hline Canaza & Rio Canaza & 1.00 & N $08^{\circ} 38^{\prime}$, W $83^{\circ} 10^{\prime}$ & 08.03 .2017 & 10 & 10 \\
\hline Caracol & Rio Caracol & 0.16 & N $08^{\circ} 39^{\prime}$, W $83^{\circ} 00^{\prime}$ & 09.03.2017 & 25 & 25 \\
\hline Claro & Rio Coto Colorado & 0.00 & $\mathrm{~N} 08^{\circ} 41^{\prime}, \mathrm{W} 83^{\circ} 06^{\prime}$ & 09.03 .2017 & 15 & 15 \\
\hline Conte & Rio Conte & 0.00 & $\mathrm{~N} 08^{\circ} 26^{\prime}, \mathrm{W} 83^{\circ} 02^{\prime}$ & 07.03 .2017 & 7 & 7 \\
\hline Palmar Norte & Rio Balsar & 0.25 & $\mathrm{~N} 08^{\circ} 59^{\prime}, \mathrm{W} 83^{\circ} 31^{\prime}$ & 11.03 .2017 & 32 & 31 \\
\hline Seco & Rio Seco & 0.08 & N $08^{\circ} 39^{\prime}, W 2^{\circ} 56^{\prime}$ & 06.03 .2017 & 25 & 23 \\
\hline Coloradito middle & Rio Coloradito & 0.27 & $\mathrm{~N} 08^{\circ} 35^{\prime}, \mathrm{W} 82^{\circ} 52^{\prime}$ & 05.03 .2017 & 37 & 36 \\
\hline Porto Grande & Rio Guineal & 0.20 & $\mathrm{~N} 09^{\circ} 01^{\prime}, \mathrm{W} 83^{\circ} 09^{\prime}$ & 01.03 .2017 & 10 & 10 \\
\hline Ceibo upstream & Rio Ceibo & 0.05 & $\mathrm{~N} 09^{\circ} 12^{\prime}, \mathrm{W} 83^{\circ} 18^{\prime}$ & 27.02 .2017 & 22 & 21 \\
\hline Bonito & Rio Bonito & 0.87 & N $08^{\circ} 43^{\prime}$, W $83^{\circ} 12^{\prime}$ & 25.03 .2018 & 15 & 14 \\
\hline Esquinas & Rio Esquinas & 0.10 & $\mathrm{~N} 08^{\circ} 44^{\prime}, \mathrm{W} 83^{\circ} 10^{\prime}$ & 24.03.2018 & 20 & 17 \\
\hline Sabalo & Rio Sabalo & 1.00 & N $08^{\circ} 52^{\prime}$, W $83^{\circ} 19^{\prime}$ & 26.03 .2018 & 15 & 15 \\
\hline Abrojo & Rio Abrojo & 0.25 & $\mathrm{~N} 08^{\circ} 37^{\prime}, \mathrm{W} 82^{\circ} 52^{\prime}$ & 23.03.2018 & 12 & 12 \\
\hline Corredor & Rio Corredor & 0.30 & N $08^{\circ} 40^{\prime}$, W $82^{\circ} 54^{\prime}$ & 23.03.2018 & 20 & 20 \\
\hline Jaballo & Quebrada Jaballo & 0.80 & N $08^{\circ} 57^{\prime}$, W $83^{\circ} 06^{\prime}$ & 16.03 .2018 & 5 & 5 \\
\hline Pavon & Rio Balsar & 0.29 & $\mathrm{~N} 09^{\circ} 00^{\prime}, \mathrm{W} 83^{\circ} 31^{\prime}$ & 26.03.2018 & 17 & 17 \\
\hline Singri & Rio Singri & 0.00 & N $08^{\circ} 59^{\prime}$, W $83^{\circ} 06^{\prime}$ & 15.03.2018 & 6 & 6 \\
\hline Tinoco & Rio Tinoco & 1.00 & $\mathrm{~N} 08^{\circ} 54^{\prime}, \mathrm{W} 83^{\circ} 22^{\prime}$ & 26.03 .2018 & 18 & 18 \\
\hline Coloradito upstream & Rio Coloradito & 0.35 & $\mathrm{~N} 08^{\circ} 35^{\prime}, \mathrm{W} 82^{\circ} 51^{\prime}$ & 25.03.2018 & 20 & 20 \\
\hline Total & & & & & 331 & 322 \\
\hline
\end{tabular}




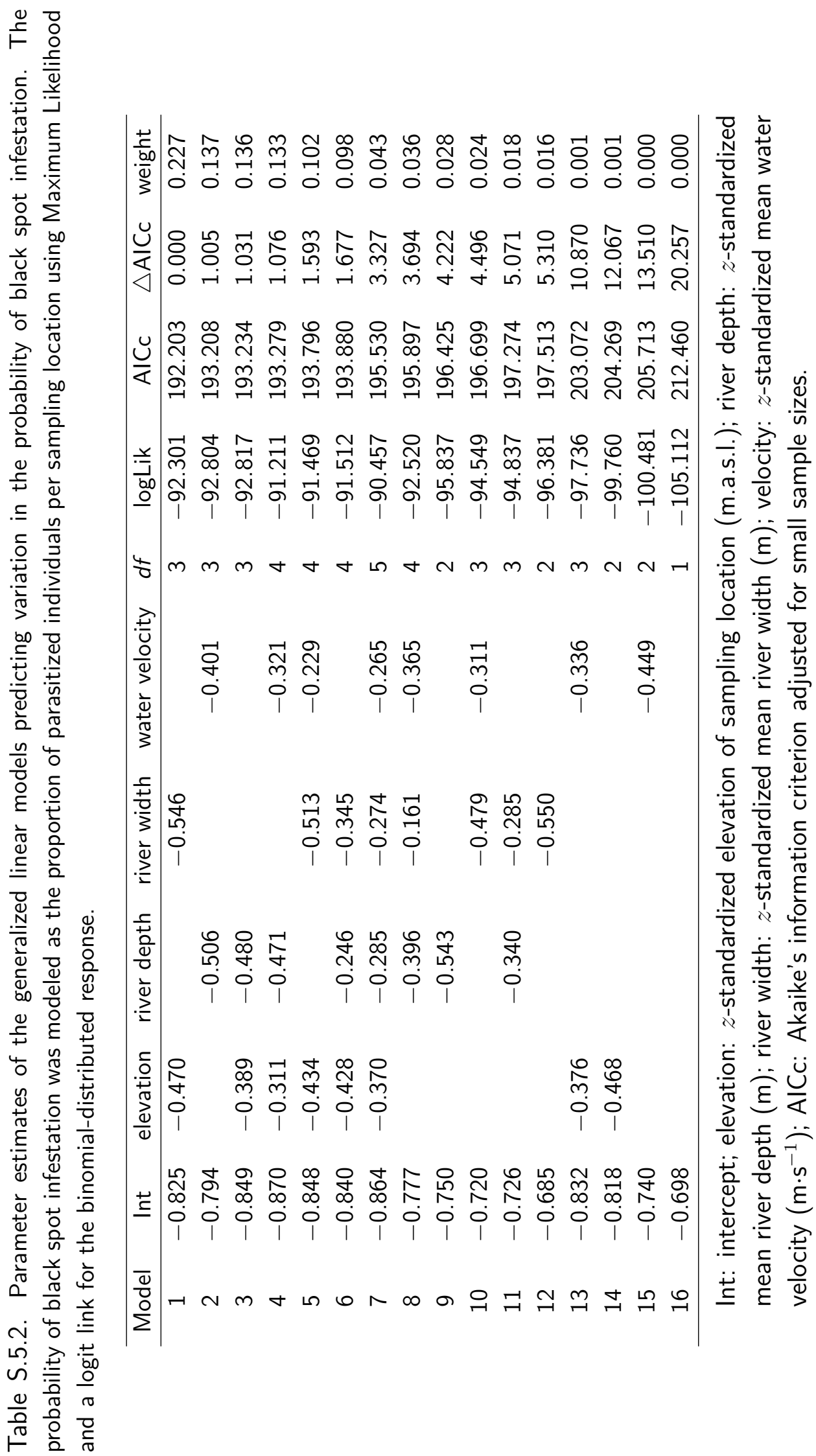


Table S.5.3. Pairwise Pearson correlation between the measured environmental variables at each sampling location.

\begin{tabular}{rcccc}
\hline & water velocity & river depth & river width & elevation \\
\hline water velocity & 1.000 & 0.217 & 0.298 & 0.410 \\
river depth & 0.217 & 1.000 & 0.821 & 0.225 \\
river width & 0.298 & 0.821 & 1.000 & 0.163 \\
elevation & 0.410 & 0.225 & 0.163 & 1.000 \\
\hline
\end{tabular}

Table S.5.4. Model-averaged parameters predicting variation in the probability of black spot infestation. The probability of black spot infestation was modeled as the proportion of parasitized individuals per sampling location using Maximum Likelihood and a logit link for the binomialdistributed response. Particularly, each parameter estimate is averaged over the models in the 'top model set' ( $\triangle \mathrm{AICc}<2$; Table $\mathrm{S} .5 .2)$ in which that predictor appears and is weighted by the summed weights of these models (Burnham \& Anderson, 2002).

\begin{tabular}{rcccccc}
\hline \multicolumn{7}{c}{ Estimates } \\
\hline$\beta$ & SE & adj. SE & $z$ & $P$ & Sig. \\
\hline intercept & -0.835 & 0.139 & 0.150 & 5.566 & 0.000 & $* * *$ \\
elevation* & -0.413 & 0.185 & 0.199 & 2.068 & 0.039 & $*$ \\
river width* & -0.492 & 0.186 & 0.199 & 2.475 & 0.013 & $*$ \\
river depth* & -0.439 & 0.176 & 0.187 & 2.351 & 0.019 & $*$ \\
water velocity* & -0.325 & 0.192 & 0.206 & 1.580 & 0.114 & n.s. \\
\hline
\end{tabular}

${ }^{*} z$-standardized; $\beta$ : regression coefficient; significant codes:

$P<0.001^{* * *},<0.01^{* *}, \leq 0.05^{*},>0.05$ n.s.

Table S.5.5. Fixed and random effects in explaining variation in parasite load (number of black spots per female) estimated in a generalized linear mixed model using Maximum Likelihood and a log link for the negative binomial-distributed response. The model was fitted using the package glmmTMB (Brooks et al., 2017).

\begin{tabular}{|c|c|c|c|c|c|c|c|}
\hline \multicolumn{8}{|c|}{ Fixed effects } \\
\hline & & & $\beta$ & SE & $z$ & $P$ & Sig. \\
\hline & & ercept & -3.339 & 1.584 & -2.109 & 0.035 & $*$ \\
\hline proportion 1 & aternal boc & $y$ fat* & -3.041 & 1.780 & -1.709 & 0.087 & n.s. \\
\hline materr & I standard & ength & 0.078 & 0.021 & 3.708 & 0.000 & $* * *$ \\
\hline \multicolumn{8}{|c|}{ Random effects } \\
\hline & Variance & \multicolumn{3}{|c|}{$2.5 \%$ confidence level } & \multicolumn{3}{|c|}{$97.5 \%$ confidence level } \\
\hline population & 0.1478 & \multicolumn{3}{|c|}{0.0004} & \multicolumn{3}{|c|}{0.6588} \\
\hline
\end{tabular}

*arcsin square-root transformed; $\beta$ : regression coefficient; significant codes: $P<0.001^{* * *},<0.01^{* *}, \leq 0.05^{*},>0.05$ n.s. 
Table S.5.6. Fixed and random effects in explaining variation in maternal life history. (a) Offspring dry mass at birth (developmental stage 45), (b) proportion offspring fat at birth, (c) egg dry mass at fertilization (developmental stage 2), (d) proportion egg fat at fertilization, (e) absolute dry reproductive allotment, (f) average brood size, (g) maternal fecundity (number embryos in all broods combined), (h) degree of superfetation, and (i) abortion incidence estimated using the MCMCglmm package (Hadfield, 2010). All traits were fitted in a multivariate (generalized) linear mixed effects model allowing for the covariance between the residuals of all responses. The default normal priors were used for the fixed effects with the expected value of 0 and variance $10^{12}$. Inverse-Wishart priors were used for the residual variances with the expected value of 1 and degree of belief of 0.02 . The number of iterations was 5,500,000, with a burnin of 500,000 and a thinning of 5000 .

(a) Offspring dry mass at birth

\begin{tabular}{rcccccc}
\hline \multicolumn{7}{c}{ Fixed effects } \\
\hline & $\beta_{\text {post.mean }}$ & $2.5 \% \mathrm{Cl}$ & $97.5 \% \mathrm{Cl}$ & eff.samp & $P_{\mathrm{MCMC}}$ & Sig. \\
intercept & 0.717 & -4.129 & 5.755 & 1181.800 & 0.754 & n.s. \\
parasite load* & -0.125 & -0.212 & -0.029 & 1073.222 & 0.014 & $*$ \\
maternal standard length & 0.062 & -0.012 & 0.137 & 1000.000 & 0.100 & n.s. \\
proportion maternal body fat $^{\dagger}$ & 9.392 & 3.697 & 15.395 & 1000.000 & 0.001 & $* * *$ \\
\hline
\end{tabular}

\begin{tabular}{lcccc}
\hline \multicolumn{5}{c}{ Random effects } \\
\hline & $\sigma_{\text {post.mean }}^{2}$ & $2.5 \% \mathrm{Cl}$ & $97.5 \% \mathrm{Cl}$ & eff.samp \\
\hline population identity & 0.6397 & 0.0048 & 1.8228 & 1000.0000 \\
\hline
\end{tabular}

\begin{tabular}{rccccccc}
\hline \multicolumn{10}{c}{ (b) Proportion offspring fat at birth } \\
\hline \multicolumn{7}{c}{ Fixed effects } \\
intercept & 0.398 & 0.291 & 0.495 & 1129.907 & 0.001 & $* * *$ \\
parasite load* & -0.001 & -0.003 & 0.000 & 1000.000 & 0.110 & n.s. \\
maternal standard length & 0.001 & -0.001 & 0.002 & 1000.000 & 0.216 & n.s. \\
proportion maternal body fat ${ }^{\dagger}$ & 0.263 & 0.136 & 0.365 & 1000.000 & 0.001 & $* * *$ \\
\hline
\end{tabular}

\begin{tabular}{ccccc}
\hline \multicolumn{4}{c}{ Random effects } \\
\hline & $\sigma_{\text {post.mean }}^{2}$ & $2.5 \% \mathrm{Cl}$ & $97.5 \% \mathrm{Cl}$ & eff.samp \\
\hline population identity & 0.0026 & 0.0011 & 0.0050 & 1000.0000 \\
\hline
\end{tabular}


(c) Egg dry mass at fertilization

\begin{tabular}{rcccccc}
\hline \multicolumn{7}{c}{ Fixed effects } \\
\hline intercept & 0.154 & 0.037 & 0.279 & 1000.000 & 0.014 & $*$ \\
parasite load* & -0.000 & -0.003 & 0.002 & 612.852 & 0.818 & n.s. \\
maternal standard length & 0.003 & 0.002 & 0.005 & 1000.000 & 0.001 & $* * *$ \\
proportion maternal body fat $^{\dagger}$ & -0.026 & -0.182 & 0.103 & 1000.000 & 0.722 & n.s. \\
\hline
\end{tabular}

\begin{tabular}{ccccc}
\hline \multicolumn{5}{c}{ Random effects } \\
\hline & $\sigma_{\text {post.mean }}^{2}$ & $2.5 \% \mathrm{Cl}$ & $97.5 \% \mathrm{Cl}$ & eff.samp \\
\hline population identity & 0.0037 & 0.0016 & 0.0069 & 1000.0000 \\
\hline
\end{tabular}

(d) Proportion egg fat at fertilization

\begin{tabular}{|c|c|c|c|c|c|c|}
\hline \multicolumn{7}{|c|}{ Fixed effects } \\
\hline & $\beta_{\text {post.mean }}$ & $2.5 \% \mathrm{Cl}$ & $97.5 \% \mathrm{Cl}$ & eff.samp & $P_{\mathrm{MCMC}}$ & Sig. \\
\hline intercept & -0.028 & -0.346 & 0.228 & 1000.000 & 0.852 & n.s. \\
\hline parasite load* & -0.001 & -0.005 & 0.004 & 1201.242 & 0.658 & n.s. \\
\hline maternal standard length & 0.003 & -0.001 & 0.007 & 1000.000 & 0.248 & n.s. \\
\hline proportion maternal body fat ${ }^{\dagger}$ & 0.244 & -0.090 & 0.535 & 1000.000 & 0.124 & n.s \\
\hline
\end{tabular}

\begin{tabular}{ccccc}
\hline \multicolumn{5}{c}{ Random effects } \\
\hline & $\sigma_{\text {post.mean }}^{2}$ & $2.5 \% \mathrm{Cl}$ & $97.5 \% \mathrm{Cl}$ & eff.samp \\
\hline population identity & 0.0047 & 0.0013 & 0.0093 & 1000.0000 \\
\hline
\end{tabular}

(e) Absolute dry reproductive allotment

\begin{tabular}{rcccccc}
\hline \multicolumn{7}{c}{ Fixed effects } \\
\hline intercept & $\beta_{\text {post.mean }}$ & $2.5 \% \mathrm{Cl}$ & $97.5 \% \mathrm{Cl}$ & eff.samp & $P_{\text {MCMC }}$ & Sig. \\
parasite load & -0.020 & -7.812 & -6.239 & 1000.000 & 0.001 & $* * *$ \\
maternal standard length & 0.069 & -0.021 & 0.006 & 1000.000 & 0.198 & n.s. \\
proportion maternal body fat $^{\dagger}$ & -0.164 & -0.988 & 0.080 & 1000.000 & 0.001 & $* * *$ \\
latest stage $^{\ddagger}$ & 0.091 & 0.078 & 0.103 & 887.257 & 0.001 & $* * *$ \\
latest stage $^{2 \S}$ & 0.141 & 0.101 & 0.180 & 1000.000 & 0.001 & $* * *$ \\
parasite load* $^{*}$ latest stage & -0.000 & -0.001 & 0.001 & 1000.000 & 0.648 & n.s. \\
\hline
\end{tabular}

\begin{tabular}{ccccc}
\hline \multicolumn{5}{c}{ Random effects } \\
\hline & $\sigma_{\text {post.mean }}^{2}$ & $2.5 \% \mathrm{Cl}$ & $97.5 \% \mathrm{Cl}$ & eff.samp \\
\hline population identity & 0.0806 & 0.0234 & 0.1661 & 909.5209 \\
\hline
\end{tabular}




\section{(f) Average brood size}

\begin{tabular}{rcccccc}
\hline \multicolumn{7}{c}{ Fixed effects } \\
\hline & $\beta_{\text {post.mean }}$ & $2.5 \% \mathrm{Cl}$ & $97.5 \% \mathrm{Cl}$ & eff.samp & $P_{\mathrm{MCMC}}$ & Sig. \\
intercept & -10.298 & -13.282 & -7.187 & 1000.000 & 0.001 & $* * *$ \\
parasite load* & -0.025 & -0.073 & 0.021 & 1101.574 & 0.288 & n.s. \\
maternal standard length & 0.316 & 0.275 & 0.359 & 1000.000 & 0.001 & $* * *$ \\
proportion maternal body fat $^{\dagger}$ & -5.020 & -7.949 & -1.567 & 1000.000 & 0.004 & $* *$ \\
\hline
\end{tabular}

\begin{tabular}{ccccc}
\hline \multicolumn{5}{c}{ Random effects } \\
\hline & $\sigma_{\text {post.mean }}^{2}$ & $2.5 \% \mathrm{Cl}$ & $97.5 \% \mathrm{Cl}$ & eff.samp \\
\hline population identity & 2.7812 & 1.0072 & 5.2264 & 1000.0000 \\
\hline
\end{tabular}

\begin{tabular}{rcccccc}
\hline \multicolumn{7}{c}{ (g) Maternal fecundity } \\
\hline \multicolumn{7}{c}{ Fixed effects } \\
\hline intercept & -1.489 & -2.187 & -0.616 & 1000.000 & 0.001 & $* * *$ \\
parasite load* & -0.003 & -0.016 & 0.007 & 1108.687 & 0.560 & n.s. \\
\hline maternal standard length & 0.053 & 0.043 & 0.064 & 1000.000 & 0.001 & $* * *$ \\
proportion maternal body fat ${ }^{\dagger}$ & -0.666 & -1.478 & 0.085 & 1000.000 & 0.096 & n.s. \\
proportion maternal body fat ${ }^{2||}$ & -0.021 & -0.067 & 0.026 & 818.957 & 0.434 & n.s. \\
latest stage & 0.031 & 0.024 & 0.039 & 897.857 & 0.001 & $* * *$ \\
\hline
\end{tabular}

\begin{tabular}{lcccc}
\hline \multicolumn{5}{c}{ Random effects } \\
\hline & $\sigma_{\text {post.mean }}^{2}$ & $2.5 \% \mathrm{Cl}$ & $97.5 \% \mathrm{Cl}$ & eff.samp \\
\hline population identity & 0.0832 & 0.0270 & 0.1640 & 1435.8285 \\
\hline
\end{tabular}

\begin{tabular}{|c|c|c|c|c|c|c|}
\hline \multicolumn{7}{|c|}{ (h) Degree of superfetation } \\
\hline \multicolumn{7}{|c|}{ Fixed effects } \\
\hline & $\beta_{\text {post.mean }}$ & $2.5 \% \mathrm{Cl}$ & $97.5 \% \mathrm{Cl}$ & eff.samp & $P_{\mathrm{MCMC}}$ & Sig. \\
\hline intercept & -0.000 & -0.837 & 0.840 & 1000.000 & 0.994 & n.s. \\
\hline parasite load* & 0.000 & -0.015 & 0.016 & 1062.766 & 0.936 & n.s. \\
\hline maternal standard length & 0.004 & -0.009 & 0.016 & 896.360 & 0.574 & n.s. \\
\hline proportion maternal body fat ${ }^{\dagger}$ & 0.003 & -0.891 & 0.883 & 1000.000 & 0.966 & n.s. \\
\hline latest stage & 0.020 & 0.009 & 0.029 & 680.016 & 0.001 & $* * *$ \\
\hline \multicolumn{7}{|c|}{ Random effects } \\
\hline & $\sigma_{\text {post.mea }}^{2}$ & $2.5 \% \mathrm{Cl}$ & $97.5 \% \mathrm{Cl}$ & eff.samp & & \\
\hline population identity & 0.0086 & 0.0014 & 0.0189 & 1000.0000 & & \\
\hline
\end{tabular}




\begin{tabular}{rcccccc}
\hline \multicolumn{7}{c}{ (i) Abortion incidence } \\
\hline \multicolumn{7}{c}{ Fixed effects } \\
\hline intercept & 0.172 & -0.199 & 0.604 & 1000.000 & 0.388 & n.s. \\
parasite load & 0.001 & -0.006 & 0.008 & 1000.000 & 0.892 & n.s. \\
maternal standard length & -0.002 & -0.007 & 0.004 & 1000.000 & 0.546 & n.s. \\
proportion maternal body fat & 0.465 & 0.028 & 0.878 & 1000.000 & 0.034 & $*$ \\
\hline
\end{tabular}

\begin{tabular}{ccccc}
\hline \multicolumn{5}{c}{ Random effects } \\
\hline & $\sigma_{\text {post.mean }}^{2}$ & $2.5 \% \mathrm{Cl}$ & $97.5 \% \mathrm{Cl}$ & eff.samp \\
\hline population identity & 0.0156 & 0.0031 & 0.0305 & 1000.0000 \\
\hline
\end{tabular}

${ }^{*}$ number of black spots per female; ${ }^{\dagger}$ arcsin square-root transformed; ${ }^{\ddagger}$ median-centered developmental stage of the most developed brood; ${ }^{\S}$ quadratic $z$-standardized developmental stage of the most developed brood; "quadratic $z$-standardized arcsin square-root transformed proportion of maternal body fat; 'developmental stage of the most developed brood; $\beta_{\text {post.mean }}$ : posterior mean of regression coefficient; $\mathrm{Cl}$ : confidence interval; eff.samp: effective sample size; $P_{\mathrm{MCMC}}$ : posterior bayesian $p$-value; significant codes: $P_{\mathrm{MCMC}} \leq 0.001^{* * *},<0.01^{* *}, \leq 0.05^{*},>0.05$ n.s.

Table S.5.7. Parameter estimates of the generalized linear model predicting variation in the probability of emerging from the box and crossing the metal ring. Whether an individual emerged from the box and crossed the metal ring was fitted as a binary variable (yes or no) using Maximum Likelihood and a logit link for the binomial-distributed response.

\begin{tabular}{rccccc}
\hline \multicolumn{7}{c}{ Estimates } & & & \\
\hline & $\beta$ & SE & $z$ & $P$ & Sig. \\
intercept & 8.511 & 3.714 & 2.292 & 0.022 & $*$ \\
standard length & -0.153 & 0.089 & -1.715 & 0.086 & n.s. \\
males & 0.806 & 1.216 & 0.663 & 0.507 & n.s. \\
parasite load* & 0.004 & 0.015 & 0.254 & 0.800 & n.s. \\
day 2 & -1.361 & 0.773 & -1.761 & 0.078 & n.s. \\
\hline
\end{tabular}

*number of black spots per female; $\beta$ : regression coefficient; significant codes: $P<0.001^{* * *},<0.01^{* *}$, $\leq 0.05^{*},>0.05$ n.s. 
Table S.5.8. Parameter estimates of the generalized linear model predicting variation in the boldness score (i.e. time taken to emerge from the box) (s) fitted using Maximum Likelihood and a log link for the quasipoisson-distributed response.

\begin{tabular}{rccccc}
\hline \multicolumn{7}{c}{ Estimates } \\
\hline SE & $t$ & $P$ & Sig. \\
\hline intercept & 3.153 & 1.120 & 2.814 & 0.007 & $* *$ \\
standard length & 0.013 & 0.028 & 0.448 & 0.656 & n.s. \\
males & 0.201 & 0.332 & 0.606 & 0.547 & n.s. \\
day 2 & 0.970 & 0.300 & 3.232 & 0.002 & $* *$ \\
parasite load* & -0.002 & 0.006 & -0.372 & 0.712 & n.s. \\
\hline${ }^{*}$ number of black spots per female; $\beta$ : regression co- \\
efficient; significant codes: $P<0.001^{* * *},<0.01^{* *}$, \\
$\leq 0.05^{*},>0.05$ n.s.
\end{tabular}

Table S.5.9. Parameter estimates of the generalized linear model predicting variation in hesitancy (s) fitted using Maximum Likelihood and a log link for the quasipoisson-distributed response.

\begin{tabular}{rccccc}
\hline \multicolumn{7}{c}{ Estimates } & & & \\
\hline & $\beta$ & SE & $t$ & $P$ & Sig. \\
\hline intercept & 2.198 & 1.293 & 1.699 & 0.097 & n.s. \\
standard length & 0.059 & 0.032 & 1.824 & 0.075 & n.s. \\
males $^{0}$ & 0.584 & 0.315 & 1.853 & 0.071 & n.s. \\
parasite load* & -0.002 & 0.006 & -0.325 & 0.747 & n.s. \\
day 2 & 0.434 & 0.263 & 1.650 & 0.107 & n.s. \\
\hline
\end{tabular}

*number of black spots per female; $\beta$ : regression coefficient; significant codes: $P<0.001^{* * *},<0.01^{* *}$, $\leq 0.05^{*},>0.05$ n.s. 



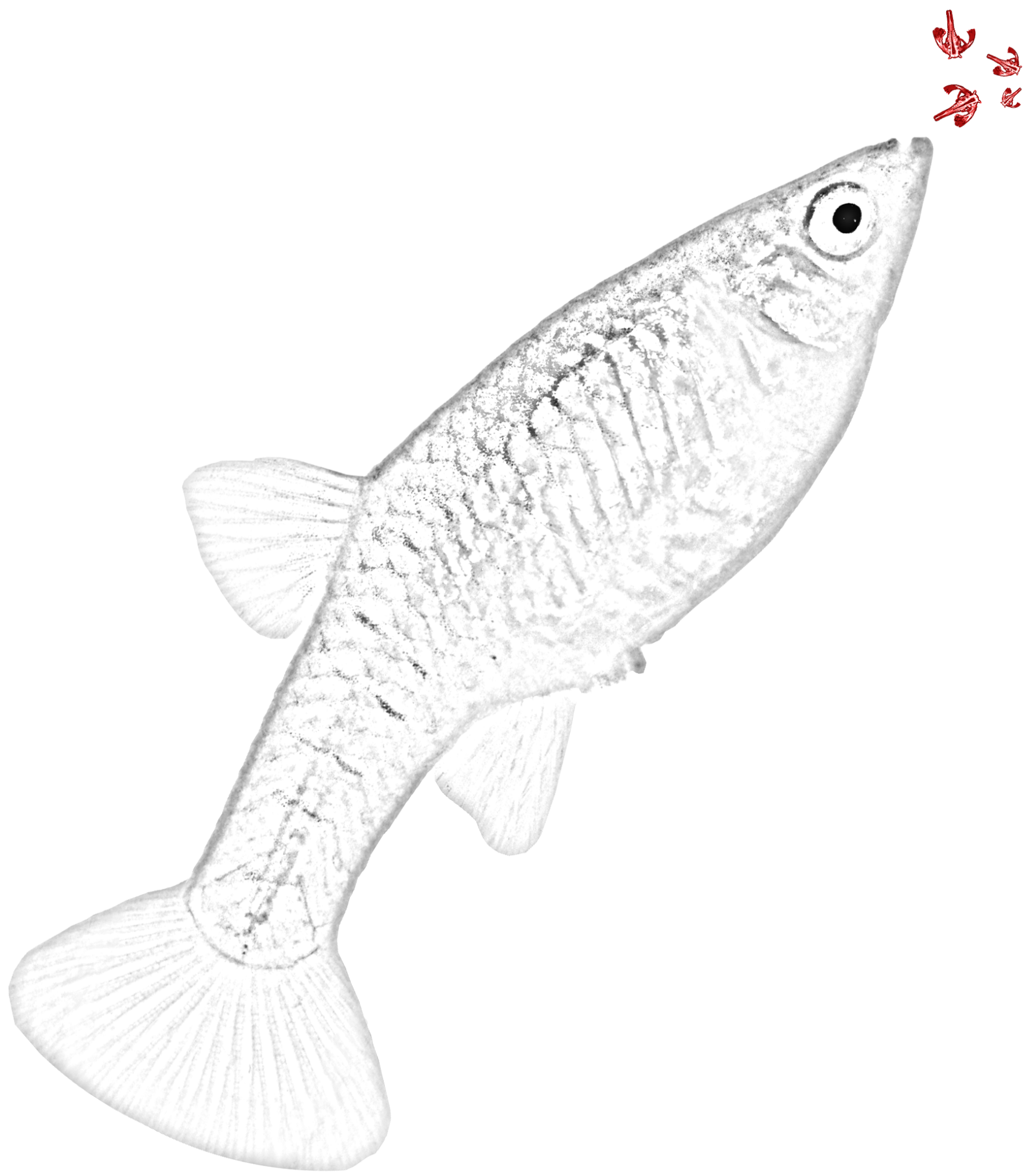




\section{Maternal food limitation during pregnancy affects offspring growth and locomotor per- formance after birth in a matrotrophic live- bearing fish}

Andres Hagmayer ${ }^{1}$, Martin J. Lankheet ${ }^{1}$, Judith Bijsterbosch ${ }^{1}$, Johan L. van Leeuwen ${ }^{1} \&$ Bart J.A. Pollux ${ }^{1}$

1 Department of Animal Sciences, Wageningen University, 6708 WD Wageningen, The Netherlands 


\section{Abstract}

The pattern of how mothers allocate resources to different functions such as maintenance, somatic growth, and reproduction can have profound implications for early life development and survival of subsequent generations. Here we examined to what extent maternal food limitation during pregnancy affects offspring size and quality at birth, as well as growth and locomotor performance after birth in the matrotrophic live-bearing fish species Phalloptychus januarius (Poeciliidae). We fed females either with a 'low-food' or 'high-food' ration during seven weeks and measured size and body fat of their $\mathrm{F}_{1}$-offspring at birth, at one week after birth, and as adults (nine months after birth). In addition, we measured fast-start escape performance of $\mathrm{F}_{1}$-offspring on the day of birth, as well as the swimming kinematics during feeding at zero, two, and seven days after birth. Finally, we quantified the size of $\mathrm{F}_{2}$-offspring during the first week after birth. We show that maternal food restriction resulted in a decrease in maternal wet mass throughout the experiment, leading to a reduced resource allocation to $\mathrm{F}_{1}$-offspring size and body fat, which was compensated only in the long-term (i.e. when adult). Although food restriction did not impact the fast-start escape performance of $\mathrm{F}_{1}$-offspring immediately after birth, it negatively affected postnatal development of locomotor performance during feeding, and thus, body condition and presumably fitness after birth. Maternal food restriction had a negative effect on offspring size at birth in the $\mathrm{F}_{2}$-generation, but the effect disappeared one week after birth. Our study suggests that matrotrophy in poeciliids is likely a maladaptive strategy in fluctuating resource environments, because sudden reductions in maternal food availability during pregnancy result in smaller offspring with a lower locomotor performance during early life.

Keywords: life-history, live-bearing, matrotrophy, placenta, placentotrophy, Poeciliidae, superfetation, viviparity

\subsection{Introduction}

How individuals allocate resources to different functions such as maintenance, somatic growth, and reproduction is crucial for their life-history (Stearns, 1992). Different allocations compete for limited resources, leading to trade-offs and a limited set of possible life-history strategies (Braendle et al., 2011).

Besides intrinsic trade-offs and constraints, resource allocation and hence life-histories are influenced by environmental factors, such as food availability (Boggs, 1992). Increased maternal food availability enables a higher energy uptake, which can be allocated to any function (van Noordwijk \& de Jong, 1986). However, when food is scarce specific functions may be prioritized over others. In lecithotrophic (i.e. yolk-feeding) live-bearing fish, adverse food conditions may reduce maternal growth, fat reserves, and fecundity, yet also increase the relative investment in offspring size (Reznick et al., 1996b; Bashey, 
2008). In this example, mothers adaptively modified offspring phenotype in preparation for predicted, adverse environmental conditions (low food availability). This response is presumably advantageous, as under competitive adverse food conditions, mothers gain fitness benefits by producing larger offspring (Parker \& Begon, 1986; Leips et al., 2013). However, as optimal offspring size is given by the offspring size-performance relationship that depends on the environment, the advantage of producing larger offspring at birth diminishes or disappears in favorable environments (Parker \& Begon, 1986; Bashey, 2008; Leips et al., 2013), and the predicted optimal strategy for the mother is to produce more numerous but smaller offspring (Jørgensen et al., 2011). If the maternal environment reliably predicts future environmental conditions, females may evolve the ability to adaptively adjust offspring phenotype at birth, based on environmental cues (Mousseau \& Fox, 1998b).

The modulation and timing of nutrient acquisition and allocation also affect life-histories (Zera \& Harshman, 2001). In matrotrophic live-bearing fish, experimental manipulations of food availability can impact the pattern of resource allocation to offspring. Instead of allocating all resources to the eggs prior to fertilization (i.e. lecithotrophy), matrotrophic species transfer nutrients to their embryos throughout gestation via a placenta (Wourms, 1981; Reznick et al., 2002; Pollux et al., 2014). Because matrotrophic species continuously supply embryos with resources, determination of brood size (i.e. number of embryos per brood) and offspring size are decoupled (Pollux \& Reznick, 2011). When resource conditions during pregnancy suddenly deteriorate, matrotrophic species may not be able to optimally provision their embryos. Because females cannot abort embryos in response to food shortage and maternal fat reserves do not fully buffer females during gestation (Reznick et al., 1996b; Banet \& Reznick, 2008; Banet et al., 2010; Pollux \& Reznick, 2011), sudden resource declines inevitably result in smaller, worse-conditioned offspring at birth. Small offspring size in low food conditions is associated with a lower survival. Therefore, matrotrophy has been argued to be maladaptive in fluctuating resource environments (Reznick et al., 1996b; Trexler \& DeAngelis, 2003; Pollux \& Reznick, 2011).

Differential resource allocation to offspring size at birth can impact postnatal development and survival (Mousseau \& Fox, 1998a). In fish, body size is linked to locomotor performance (Gibb et al., 2006). Smaller offspring perform worse on fast-start escapes (Dial et al., 2016), presumably decreasing survival probability in environments with high predation risks. Prey-capture abilities immediately after birth also heavily depend on swimming performance. New-born live-bearing fish are super-precocial, having functional prey-capture abilities at birth and relying on active exogenous feeding after birth (Lankheet et al., 2016). Moreover, they rapidly develop the visuo-motor skills required for prey-capture during the first days after birth and effectively improve their success rate, promoting food uptake and survival (Lankheet et al., 2016). Offspring size thus affects locomotor performance and prey-capturing abilities after birth. However, it is still unknown to what extent restricted maternal food availability during pregnancy affects offspring swimming performance in fast-start escapes and in prey-capture after birth. 
Because offspring size and performance influence fitness, selection might favor mechanisms that mitigate negative effects of poor nutrition during early development. Compensatory growth or the ability to increase growth rate above 'routine' rates, may be an adaptive strategy to fully or even overcompensate body size in response to increased food availability following a period of growth restriction (Metcalfe \& Monaghan, 2001). This was shown in juvenile guppies (Poecilia reticulata) that could 'catch-up' their body size by increasing growth rate after a food-restriction period (Auer, 2010). Compensatory growth is thus a potential adaptive mechanism that may enhance the resilience of placental species against the negative fitness consequences of being born small when maternal resources are scarce. However, empirical evidence for compensatory mechanisms after birth and the consequences for offspring performance in placental species is still lacking.

Here, we examine to what extent maternal food limitation during pregnancy affects growth and locomotor performance of $\mathrm{F}_{1}$-offspring after birth in the matrotrophic fish species Phalloptychus januarius (family Poeciliidae; Hensel, 1868). Specifically, we measured: (i) size and body fat of the $\mathrm{F}_{1}$-offspring at different ages (i.e. 0 days, 7 days, and 9 months old), (ii) fast-start escape performance at birth, and (iii) swimming kinematics while feeding during the first week after birth, to quantify immediate and lasting effects of food limitation during pregnancy on offspring size, quality, and locomotor performance. As a proxy for locomotor performance, we focused on mean and maximum speed and acceleration of fast-starts and feeding actions. Finally, we measured (iv) the size of $\mathrm{F}_{2}$-offspring during their first week after birth to reveal possible persistent effects in subsequent generations. By combining the different measurements, we reveal consequences of maternal food limitation for life-history variation, potential compensatory mechanisms against insufficient maternal resource provisioning, and its implications for the quality and performance of subsequent generations in a placental species.

\subsection{Material and methods}

\subsubsection{Maternal food treatment during gestation}

We conducted a 7-week experiment to study the effects of maternal resource limitation during pregnancy by subjecting females to either a 'low-food' (LF) or 'high-food' (HF) ration, starting after week 1 (Fig. 6.1a). Prior to the experiment, we set up 50 8-L aquaria, each containing one mature male and female P. januarius. The fish were fed ad libitum until the start of the experiment, at which time the males were removed. Females were re-mated once overnight, after three weeks, to ensure a sufficient supply of sperm. There was a fourfold difference in food quantity between the LF and HF treatments, consisting of either $25 \mu \mathrm{l}(\mathrm{LF})$ or $100 \mu \mathrm{l}(\mathrm{HF})$ liver paste in the morning and $25 \mu \mathrm{l}$ or $100 \mu \mathrm{l}$ of newlyhatched brine shrimp (Artemia salina) in the afternoon. Previous experiments indicated that the LF rations were sufficient to sustain reproduction while the HF rations were close to ad libitum feeding (Pollux \& Reznick, 2011). We measured female body length (to the 
nearest $\mathrm{mm}$ ) and wet mass (to the nearest $0.1 \mathrm{mg}$ ) at the beginning of the food treatment (i.e. after week 1) and at the end of the experiment (i.e. after week 7; Fig. 6.1a).

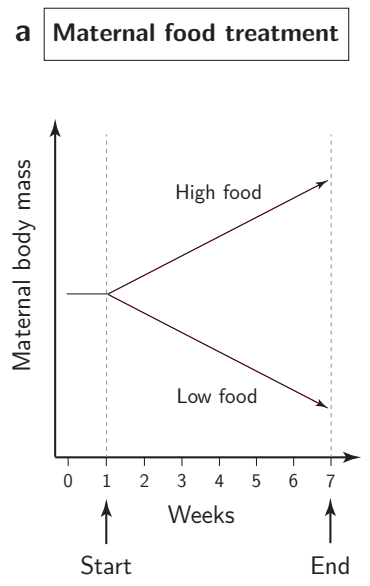

e Measurement of $F_{2}$-offspring

$\mathrm{F}_{1}$-offspring that were reared to maturity were allowed to randomly mate. The were allow $\mathrm{F}_{\text {- }}$ to repring were collected and produced $F^{2}$ frspring were collected and measuring body size after birth:

\begin{tabular}{lccc} 
Measurement & day 0 & day 2 & day 7 \\
\hline Cross-sectional & $\checkmark$ & $\checkmark$ & $\checkmark$
\end{tabular} body area

Note: due to ethical restrictions, the mass and body fat of $\mathrm{F}_{2}$-offspring could not be measured. Instead, body size was quantified as the average cross-sectional quantified as the average cross-sectional
body area measured from the dorsal side in live animals in the experimental set-up used to quantify swimming kinematics.

Fig. 6.1. Overview of the experimental design. a 7-week maternal food treatment with a timeline of the various measurements on $F_{1}$-offspring $\mathbf{b}$ at birth, $\mathbf{c}$ during their first week after birth, and d nine months after birth. e Timeline of the measurements on $F_{2}$-offspring during their first week after birth.

\subsubsection{Collection of $F_{1}$-offspring for various measurements}

Experimental tanks were checked daily for the presence of new-born offspring. These were subsequently used for various experimental procedures (Fig. 6.1): one randomly selected subset of offspring was used to measure dry mass and body fat (day 0 and 7) using established protocols (Supplementary Methods S.6.1.1). A second subset was used to quantify the fast-start escape performance (on day 0) and swimming kinematics during feeding (on day 0,2 , and 7) (see below for more details; Fig. 6.1b,c). A third subset was collected during week 7 and reared from birth to sexual maturity (in 40-L stock tanks under ad libitum food). They were allowed to freely breed within their own treatment group to produce $\mathrm{F}_{2}$-offspring. After 9 months, the $\mathrm{F}_{1}$-adults were euthanized and preserved to measure dry mass and body fat (Fig. 6.1d). Due to ethical restrictions, the dry mass and body fat of $\mathrm{F}_{2}$-offspring could not be measured. Instead, growth was quantified as the average projected body area measured from the dorsal side (Supplementary Methods S.6.1.2, S.6.1.3; Fig. 6.1e).

\subsubsection{Locomotor performance of $F_{1}$-offspring}

Individual fish were isolated in Petri dishes $(\oslash 55 \mathrm{~mm})$ and stored in an incubator at $24^{\circ} \mathrm{C}$. The fish were subsequently transferred to an experimental set-up to record either 
the (i) fast-start escape performance on day 0, or (ii) swimming kinematics during feeding on day 0,2 , and 7 . The fish were filmed from the dorsal side against an array of LEDs behind a white, translucent plate using a high-speed video camera (Supplementary Methods S.6.1.2). The water level was kept at $5 \mathrm{~mm}$ to minimize vertical movements of the fish (average body length of new-born offspring: $\sim 7 \mathrm{~mm}$ ). Following the performance measurements, the fish were euthanized and preserved to measure dry mass and fat content (Supplementary Methods S.6.1.1).

a
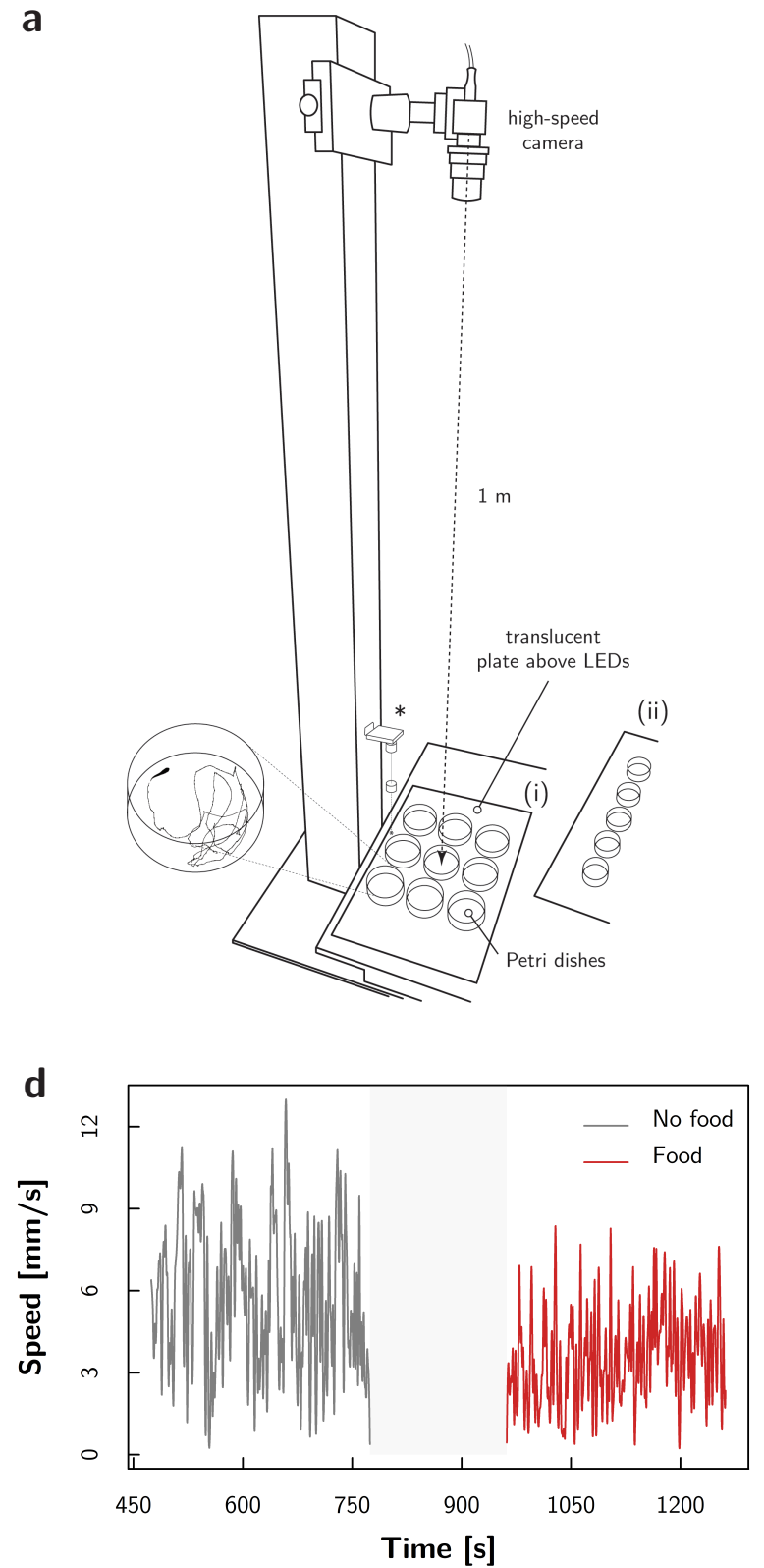
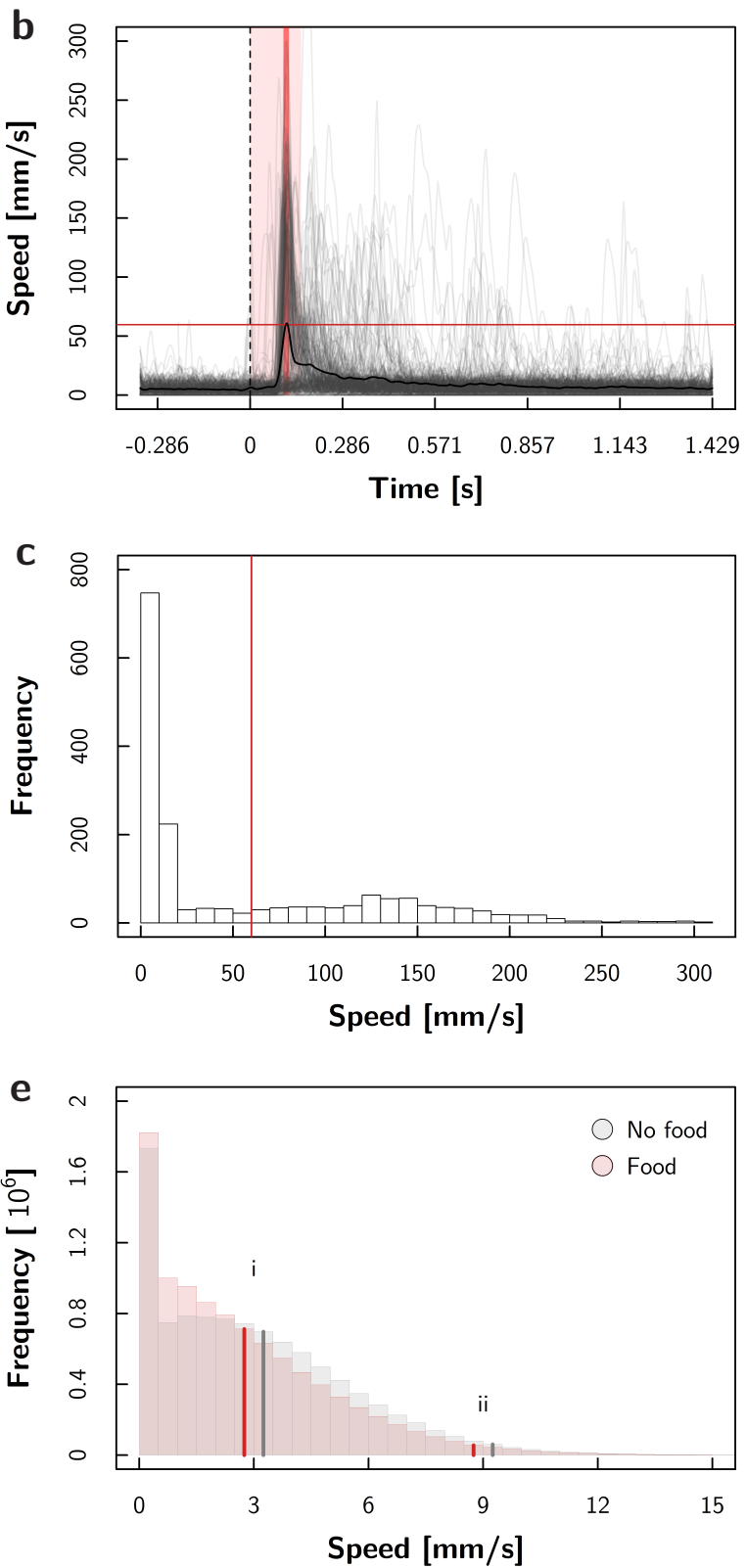
Fig. 6.2. a Experimental set-up to record either (i) swimming kinematics during feeding, or (ii) fast-start escape performance. Fish were high-speed filmed from the dorsal side. For (i), a maximum of nine Petri dishes, each with a single fish, were placed on pre-defined positions on the plate (arranged in a $3 \times 3$ pattern). For (ii), a maximum of five dishes were placed on the plate (arranged in a $1 \times 5$ pattern). Fast-start escapes were initiated by dropping a weight on the plate $(*)$. b Speed profiles of all fast-start escapes. Dashed vertical line indicates the time at which the weight is released to trigger an escape maneuver. Solid black curve shows the overall mean speed. Mean and maximum performance parameters were extracted from a period that was sufficiently long to perform a fast-start (light red rectangle). Fish were considered as having 'responded' if their speed during a 7 frame window around the maximum of the overall mean speed (thick red line) exceeded the threshold of $60 \mathrm{~mm} \cdot \mathrm{s}^{-1}$ (thin red line). c Histogram of all instant fast-start speeds observed in the light red rectangle shown in $\mathbf{b}$. The histogram shows two peaks, which correspond to individuals that have either responded to the stimulus or not. The response threshold for a fast-start (thin red line in $\mathbf{b}$ and $\mathbf{c}$ ) was defined as the speed observed at the minimum frequency in between the two peaks $\left(60 \mathrm{~mm} \cdot \mathrm{s}^{-1}\right)$. d Example time trace of speed during an individual measurement of swimming kinematics while feeding. Mean and maximum locomotor performance parameters were extracted from the 5 min control period (grey line; no food available), and 5 minutes with available food (red line). The period during which food was supplied (grey rectangle) was filtered out to remove disturbances due to the experimenter. e Histogram of all instant speeds observed during the measurements of swimming kinematics while feeding. Grey: 5 min control period (no food present); red: 5 min feeding period. Solid lines correspond to the (i) overall mean speed and (ii) mean of speeds above $95 \%$ quantile.

\subsubsection{Fast-start escape response}

Throughout the experiment up to 15 offspring were collected each Monday and Thursday (if available) to measure escape performance at birth (Fig. 6.1b). For this, the fish were transferred to smaller Petri dishes $(\oslash 35 \mathrm{~mm})$. A maximum of five dishes, each containing a single fish, were simultaneously filmed. The fast-start escape maneuver was initiated after a 5 min acclimation period by dropping a weight on the plate. Fish were allowed to recover for $5 \mathrm{~min}$, before initiating a second and third fast-start.

\subsubsection{Swimming kinematics during feeding}

Throughout the experiment up to 18 offspring were collected each Tuesday and Wednesday (if available) to measure swimming kinematics during feeding (Fig. 6.1b). The fish were held in the incubator at a 12L:12D cycle for one week and fed daily ad libitum with newly-hatched Artemia. Swimming kinematics during feeding were measured at day 0, 2, and 7 (Fig. 6.1c). A maximum of nine dishes, each containing a single fish, were simultaneously filmed (Fig. 6.2a). After an initial 5 min acclimation period, the swimming kinematics were first recorded for $5 \mathrm{~min}$ without food present (control). Subsequently, 30 newly-hatched Artemia were added to each Petri dish to trigger feeding responses. The swimming kinematics were recorded for another $5 \mathrm{~min}$. The resolution in our videos was too low to track individual Artemia, therefore we lack information about prey-capture 
success of individual fish.

\subsubsection{Video analysis}

We used an in-house developed Python program to track the fish's silhouette in real-time and to automatically extract the location of the center of mass through time. Using the fish's position, we calculated swimming speed and linear acceleration (Supplementary Methods S.6.1.4). As a proxy for locomotor performance, we extracted the mean and maximum speed and acceleration for each fast-start and food response. For fast-starts, we used a period after releasing the weight, which was sufficiently long to capture the response $(0-0.157 \mathrm{~s}$; Fig. $6.2 \mathrm{~b})$. Response intervals thus included a baseline during the time it took for the weight to hit the plate. Speed profiles were considered as fast-start responses if the speed during a 7 frame $(0.103-0.120 \mathrm{~s})$ window around the maximum of the overall mean speed exceeded the threshold of $60 \mathrm{~mm} \cdot \mathrm{s}^{-1}$ (Fig. 6.2b,c). For food responses, we used the $5 \mathrm{~min}$ control period (no Artemia present), as well as the $5 \mathrm{~min}$ feeding period (Fig. 6.2d). Because extreme values for speed and acceleration are relatively sensitive to measurement noise, we defined the maximum speed and acceleration as the mean of the values above the $95 \%$ quantile, rather than the actual maximum values (Fig. $6.2 \mathrm{e})$.

\subsubsection{Statistical analysis}

All estimations were carried out in a Bayesian framework using the MCMCglmm package (Hadfield, 2010) in R v 3.5 (R Core Team, 2020). Multivariate models allowed for the covariance between the residuals of all responses. Convergence was assessed by visual examination of the traces and the autocorrelations of the parameter chain was checked to be less than 0.1 .

To identify potential effects of maternal food limitation on maternal wet mass and standard length, both traits were fitted in a bivariate LMM. Fixed effects included treatment (LF or HF), experimental day (9 or 51), as well as treatment $\times$ day. Maternal identity was fitted as random intercept to correct for maternal variance sources not accounted for by the fixed effects. Females that were not pregnant at the end of the experiment $(n=2)$ or that died before $(n=3)$, were excluded from the analysis. In addition, maternal fecundity was fitted in a GLMM using a log link for the Poisson-distributed response. Fixed effects included treatment, experimental week (1-7), as well as treatment $\times$ week. Maternal identity was fitted as random intercept (see above).

The effects of maternal food limitation on (i) $\mathrm{F}_{1}$-offspring and (ii) $\mathrm{F}_{1}$-adult phenotypic traits (dry mass, lean mass, and body fat) were analyzed by fitting all traits in a multivariate $\mathrm{L}(\mathrm{M}) \mathrm{M}$ as a function of treatment (LF or HF). For (i), additional fixed effects included experimental day (day), day ${ }^{2}$, age ( 0 or 7 days), treatment $\times$ day, and treatment $\times$ age. Another fixed effect specified whether the offspring were found alive or dead. The probability of finding alive offspring significantly decreased throughout the 
experiment $\left(\beta_{\text {post.mean }}=-0.065, P_{\mathrm{MCMC}}=0.001\right)$, but did not differ between food treatments $\left(\beta_{\text {post.mean }}=0.008, P_{\mathrm{MCMC}}=0.732\right.$; Table S.6.1). The causes of the increasing frequency of dead offspring is unclear and warrants further research. Maternal identity was fitted as random intercept (see above). For (ii), additional fixed effects were sex (female or male), as well as treatment $\times$ sex. To optimize normality and homoscedasticity of the model residuals, body fat was square-root transformed.

For fast-start escapes we first analyzed the probability of an individual to 'respond', which is used as a proxy for the behavioral propensity to react to the startle stimulus. To model the potential effects of treatment, the individual's response (yes or no) was fitted as a function of treatment ( $\mathrm{LF}$ or $\mathrm{HF}$ ), experimental day, as well as treatment $\times$ day in a GLMM using a logit link for the binomial-distributed response. Secondly, the mean and maximum speed and acceleration (all ln-transformed) of identified responses were fitted in a multivariate LMM. Fixed effects were treatment, experimental day, as well as treatment $\times$ day. In both models, maternal identity and Petri dish position were fitted as random intercepts. The latter accounts for potential effects of the Petri dish position relative to the camera and stimulus (i.e. location of weight drop). Moreover, offspring and replicate trial identity were fitted as random intercepts to account for pseudo-replication and for variation through habituation to the stimulus, respectively.

Likewise, the effects of maternal food restriction on swimming kinematics during feeding were analyzed by fitting the mean and maximum speed and acceleration (all lntransformed) in a multivariate LMM. To specifically quantify the effect of food supply, the model was fitted to the locomotor performance parameter extracted during the (i) 5 min control period (no food supply) and (ii) 5 min feeding period. To reduce model complexity, data from the two periods were analyzed in separate models. Fixed effects included treatment (LF or HF), experimental day, age (0, 2, or 7 days), treatment $\times$ day, and treatment×age. Maternal and offspring identity, as well as Petri dish position were fitted as random intercepts (see above).

Finally, to quantify the implications of maternal food restriction for $\mathrm{F}_{2}$-offspring size, the cross-sectional body area of $\mathrm{F}_{2}$-offspring was fitted in a LMM as a function of treatment (LF or $\mathrm{HF}$ ), age $(0,2$, or 7 days), and treatment $\times$ age. Offspring identity was fitted as random intercept to account for pseudo-replication.

\subsection{Results}

\subsubsection{Maternal length, wet mass, and fecundity}

At the beginning of the food treatment, maternal standard length (SL) and wet mass did not significantly differ between LF and HF females (SL: $\beta_{\text {post.mean }}=-0.630$, $P_{\mathrm{MCMC}}=0.270$; wet mass: $\beta_{\text {post.mean }}=-0.003, P_{\mathrm{MCMC}}=0.918$; Table S.6.2; Fig. 6.3) . LF and HF females both showed an increase in SL and a decrease in wet mass throughout the experiment (Fig. 6.3a,b). LF females lost relatively more mass compared to HF 
females (0.05 vs. $0.01 \mathrm{~g}$; Fig. 6.3b), however, the interaction between food treatment and experimental day was not significant $\left(\beta_{\text {post.mean }}=-0.042, P_{\mathrm{MCMC}}=0.344\right.$; Table S.6.2). Finally, maternal fecundity did not significantly change throughout the experiment $\left(\beta_{\text {post.mean }}=0.021, P_{\mathrm{MCMC}}=0.374\right.$; Table S.6.3), and did not differ between food treatments $\left(\beta_{\text {post.mean }}=0.006, P_{\mathrm{MCMC}}=0.882 ;\right.$ Table S.6.3; Fig. S.6.1 $)$.

\section{a}
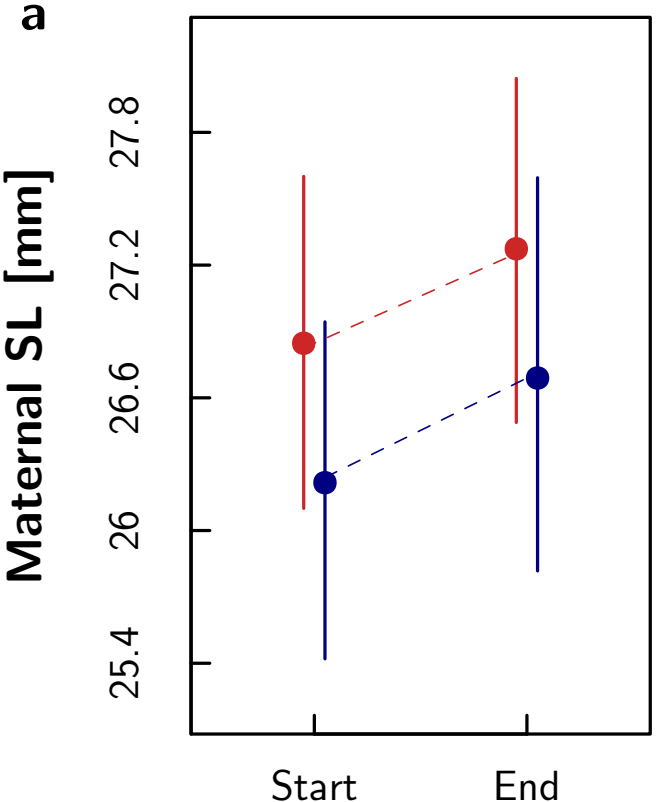

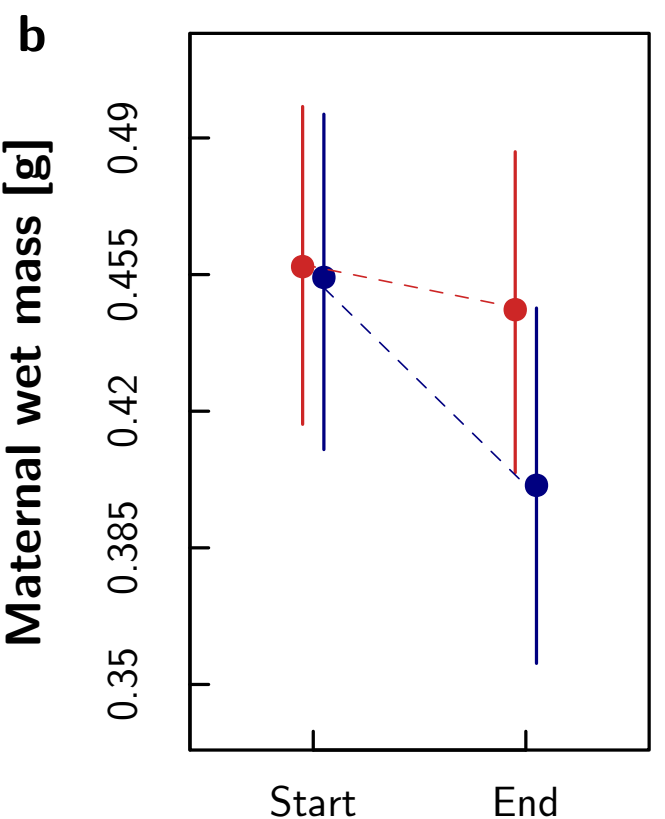

Start End

\section{Maternal food treatment}

Fig. 6.3. a Maternal standard length $(\mathrm{SL})$ and $\mathbf{b}$ wet mass $( \pm 95 \% \mathrm{Cl})$ at the start of the food treatment (after week 1) and at the end of the experiment (after week 7 ) estimated in the model described in Table S.6.2 $\left(n_{\mathrm{LF}}=22, n_{\mathrm{HF}}=23\right)$. Blue: low food; red: high food. Dashed lines correspond to linear fits throughout the posterior samples of a given food treatment.

\subsection{2 $F_{1}$-offspring size and body composition at birth}

The dry mass and lean mass of $\mathrm{F}_{1}$-offspring at birth significantly decreased throughout the 7 -week food treatment in both LF (dry mass: $\beta_{\text {post.mean }}=-0.010, P_{\mathrm{MCMC}}<0.001$; lean mass: $\left.\beta_{\text {post.mean }}=-0.008, P_{\mathrm{MCMC}}<0.001\right)$ and $\mathrm{HF}$ females $\left(\right.$ dry mass: $\beta_{\text {post.mean }}=$ $-0.003, P_{\mathrm{MCMC}}=0.012$; lean mass: $\left.\beta_{\text {post.mean }}=-0.002, P_{\mathrm{MCMC}}=0.038\right)$, however, this decrease was stronger in LF females (Table S.6.4; Fig. 6.4a,b). As a result, the $\mathrm{F}_{1}$-offspring born at the end of the experiment (i.e. experimental week 7 ) were significantly lighter and leaner for LF than HF females (dry mass: $\beta_{\text {post.mean }}=-0.251, P_{\mathrm{MCMC}}<0.001$; lean mass: $\beta_{\text {post.mean }}=-0.228, P_{\mathrm{MCMC}}<0.001 ;$ Fig. $\left.6.4 \mathrm{a}, \mathrm{b}\right)$. Offspring body fat significantly decreased throughout the experiment $\left(\beta_{\text {post.mean }}=-0.001, P_{\mathrm{MCMC}}=0.010 ;\right.$ S.6.4; Fig. $6.4 \mathrm{c})$, but did not differ between LF and HF females $\left(\beta_{\text {post.mean }}=-0.001, P_{\mathrm{MCMC}}=0.110\right.$; Table S.6.4). 


\subsection{3 $F_{1}$-offspring size and body composition seven days after birth}

Fish held in the laboratory for one week to measure swimming kinematics during feeding, were additionally used to study growth after birth. The difference in dry mass, lean mass, and body fat at birth observed at the end of the experiment persisted during the first week after birth (Fig. 6.4d-f). The body fat of offspring from HF females slightly increased during the first week after birth relative to offspring from LF females, resulting in a significantly different body fat of one-week-old offspring $\left(\beta_{\text {post.mean }}=4.1 \times 10^{-2}\right.$, $P_{\mathrm{MCMC}}=0.040 ;$ Fig. 6.4f).
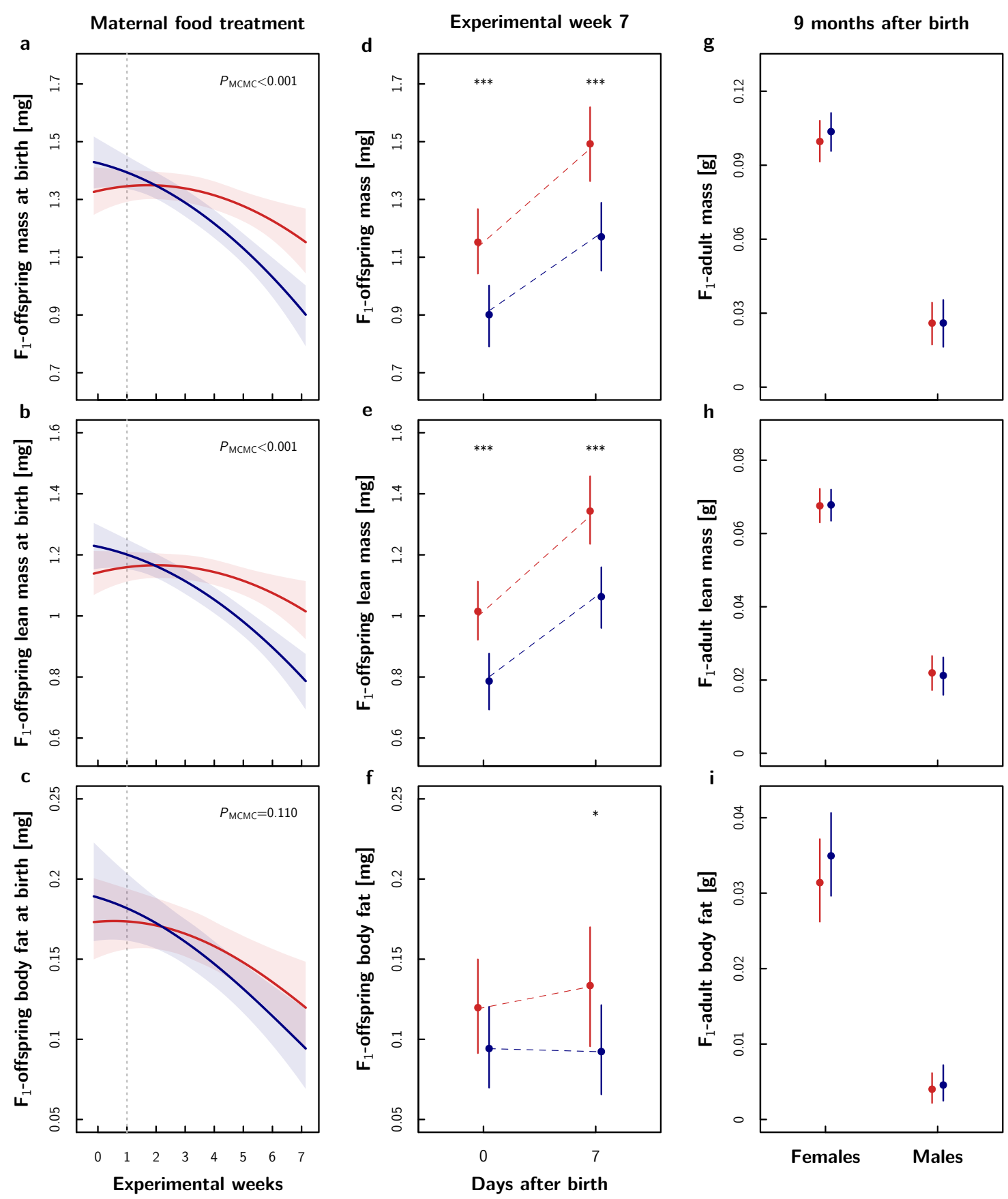
Fig. 6.4. a-f Dry mass, lean dry mass, and body fat $( \pm 95 \% \mathrm{Cl})$ of $\mathrm{F}_{1}$-offspring estimated in the model described in Table S.6.4 $\left(n_{\mathrm{LF}}=300, n_{\mathrm{HF}}=287\right)$. a-c Dry mass, lean dry mass, and body fat at birth of offspring born during the 7-week maternal food treatment from LF (blue) and HF (red) females. Dashed vertical line corresponds to the start of the food treatment. $P_{\text {MCMC-values }}$ for the interaction between experimental day and treatment are given at the top. $\mathbf{d}-\mathbf{f}$ Increase in offspring dry mass, lean dry mass, and body fat during the first week after birth, predicted for offspring that were born at the end of the 7-week experiment for both food treatments. Estimates are based on fish that were held in the laboratory for one week to measure swimming kinematics during feeding. Dashed lines represent linear fits throughout the posterior samples of a given food treatment. Significant codes: $P_{\mathrm{MCMC}} \leq 0.001^{* * *},<0.01^{* *}, \leq 0.05^{*},>0.05$ n.s. g Dry mass, $\mathbf{h}$ lean dry mass, and $\mathbf{i}$ body fat $( \pm 95 \% \mathrm{Cl})$ of adult (9 months after birth) $\mathrm{F}_{1}$-offspring born at the end of the experiment, as a function of sex (female or male) and food treatment estimated in the model described in Table S.6.5 $\left(n_{\mathrm{LF}}=19, n_{\mathrm{HF}}=20\right)$.

\subsection{4 $F_{1}$-adult size and body composition}

Whereas, as expected, adult size and body composition differed significantly between $\mathrm{F}_{1}$-males and $\mathrm{F}_{1}$-females (dry mass: $\beta_{\text {post.mean }}=0.076, P_{\mathrm{MCMC}}<0.001$; lean mass: $\beta_{\text {post.mean }}=0.046, P_{\mathrm{MCMC}}<0.001$; body fat: $\left.\beta_{\text {post.mean }}=0.029, P_{\mathrm{MCMC}}<0.001\right)$, the differences in dry mass, lean mass, and body fat at birth observed at the end of the experiment between food treatments had disappeared in the adult stage for both males and females (dry mass ${ }_{\varphi}: \beta_{\text {post.mean }}=4 \times 10^{-3}, P_{\mathrm{MCMC}}=0.496$; lean mass : $_{\text {: }}$ $\beta_{\text {post.mean }}=0.2 \times 10^{-3}, P_{\mathrm{MCMC}}=0.964 ;$ body fat $_{\phi}: \beta_{\text {post.mean }}=3.5 \times 10^{-3}, P_{\mathrm{MCMC}}=0.388$; dry $\operatorname{mass}_{0^{7}}: \beta_{\text {post.mean }}=0.5 \times 10^{-4}, P_{\mathrm{MCMC}}=0.980$; lean mass ${ }_{0^{\gamma}}: \beta_{\text {post.mean }}=-7.2 \times 10^{-4}$, $P_{\mathrm{MCMC}}=0.828$; body fat ${ }_{\sigma^{*}}: \beta_{\text {post.mean }}=5.6 \times 10^{-4}, P_{\mathrm{MCMC}}=0.714$; Table S.6.5; Fig. $6.4 \mathrm{~g}-\mathrm{i})$.

\subsection{5 $F_{1}$-offspring fast-start escape response at birth}

The probability of offspring to respond to the stimulus tended to increase throughout the 7 -week food treatment, though not significantly $\left(\beta_{\text {post.mean }}=0.044, P_{\mathrm{MCMC}}=0.056\right.$; Table S.6.6). This increase was similar in both food treatments $\left(\beta_{\text {post.mean }}=-0.010, P_{\mathrm{MCMC}}=\right.$ 0.796; Table S.6.6; Fig. 6.5a). Likewise, the mean and maximum speed and acceleration during the fast-start did not significantly change throughout the experiment, nor did it significantly differ between both food treatments (Table S.6.7; Fig. 6.5b-e). 
a

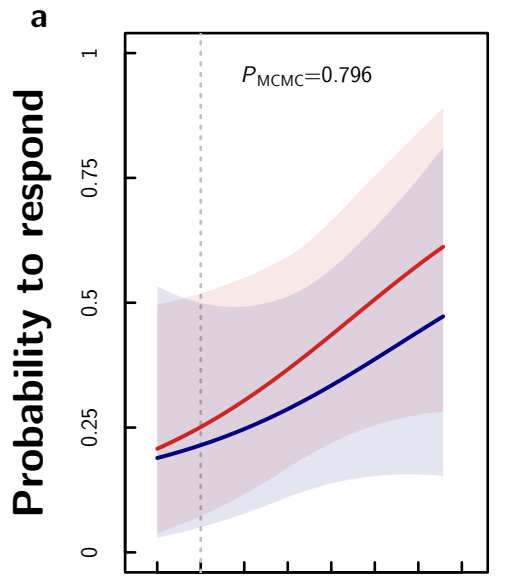

$$
\text { d }
$$

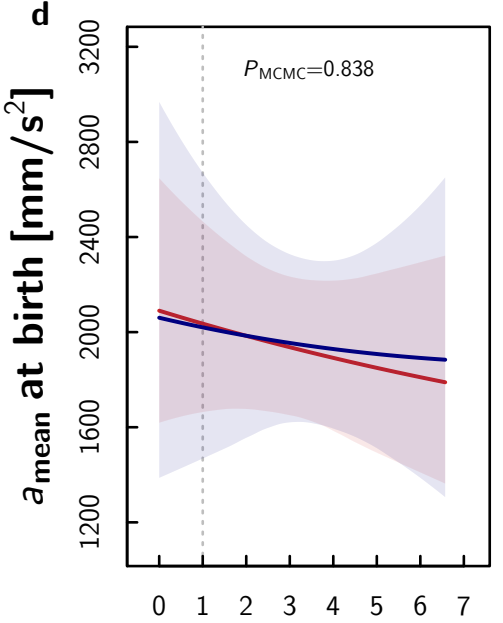

Experimental weeks b

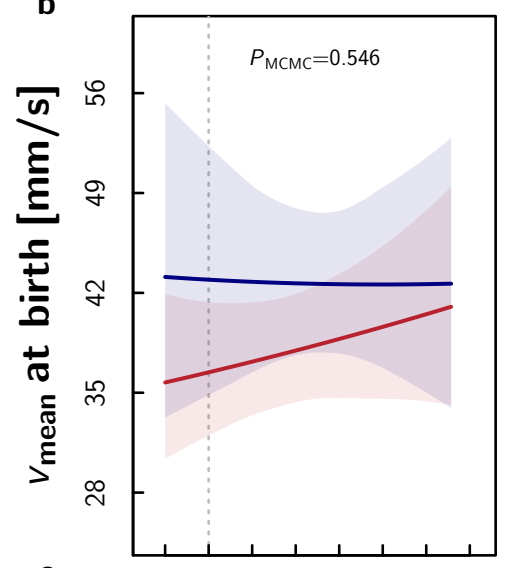

e

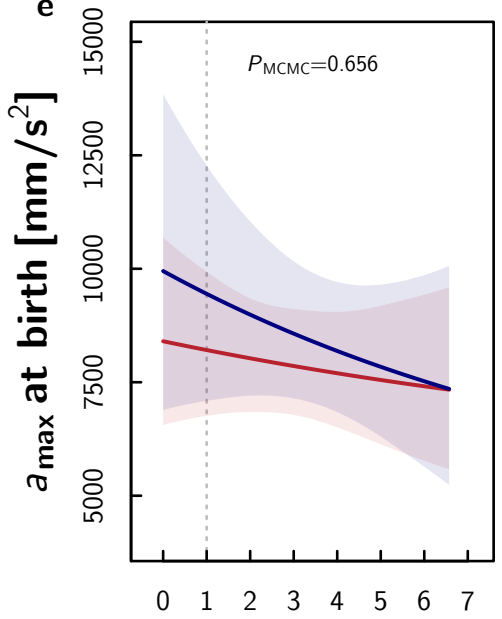

Experimental weeks

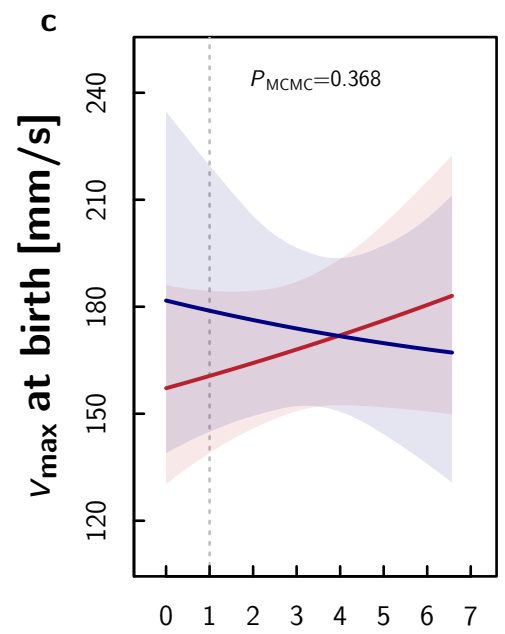

Experimental weeks

Fig. 6.5. a Probability of new-born offspring to respond to the stimulus during fast-start trials ( \pm $95 \% \mathrm{Cl})$ estimated in the model described in Table S.6.6 $\left(n_{\mathrm{LF}}=94, n_{\mathrm{HF}}=113\right)$. The responsiveness is predicted for both food treatments (blue: low food; red: high food) and throughout the experiment. Dashed vertical line corresponds to the start of the food treatment. $P_{\mathrm{MCMC}}-$ value for the interaction between experimental day and food treatment is given at the top. $\mathbf{b}-\mathbf{e}$ Swimming performance of new-born offspring during fast-start escape response estimated in the model described in Table S.6.7 $\left(n_{\mathrm{LF}}=24, n_{\mathrm{HF}}=31\right)$. New-borns were collected weekly to assess the fast-start escape response throughout the 7-week maternal food treatment (see Fig. 6.1b). b Mean speed, c maximum speed, $\mathbf{d}$ mean acceleration, and e maximum acceleration $( \pm 95 \% \mathrm{Cl})$ at birth are predicted for both food treatments (blue: low food; red: high food) and throughout the experiment. Dashed vertical line corresponds to the start of the food treatment. $P_{\mathrm{MCMC}}$-values for the interaction between experimental day and treatment are given at the top. 


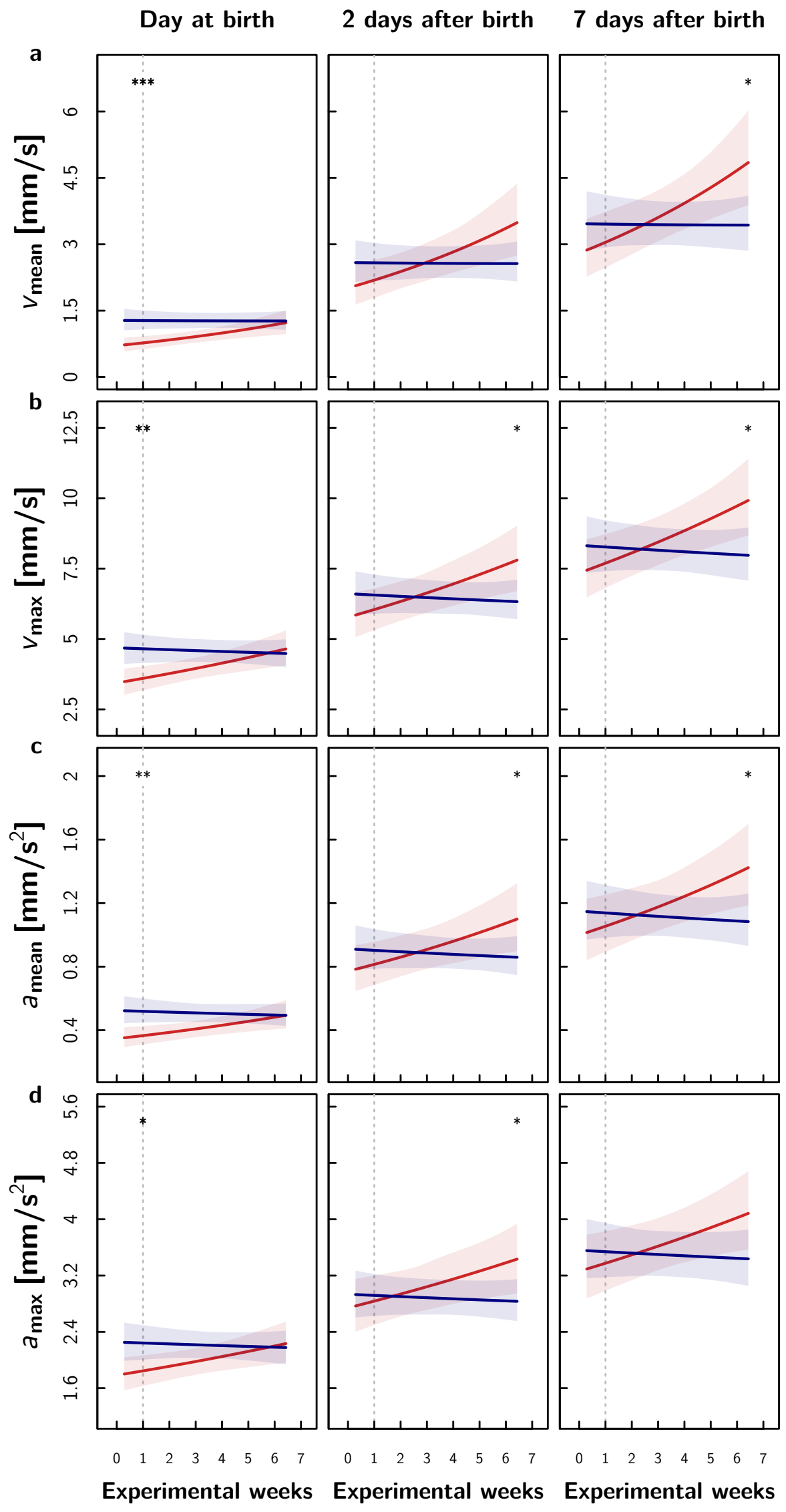


Fig. 6.6. Swimming kinematics during feeding estimated in the model described in Table S.6.8 $\left(n_{\mathrm{LF}}=183, n_{\mathrm{HF}}=135\right)$. New-borns were collected weekly to assess swimming kinematics throughout the 7-week maternal food treatment (see Fig. 6.1b,c). a Mean speed, b maximum speed, c mean acceleration, and $\mathbf{d}$ maximum acceleration $( \pm 95 \% \mathrm{Cl})$ are predicted throughout the experiment for both food treatments (blue: low food; red: high food), as well as a function of age (left panels: 0 days old; middle panels: 2 days old; right panels: 7 days old). Dashed vertical line corresponds to the start of the food treatment. Significance codes are given at the top for the difference between both food treatments at the start of the food treatment and at the end of the experiment. Significant codes: $P_{\mathrm{MCMC}} \leq 0.001^{* * *},<0.01^{* *}, \leq 0.05^{*},>0.05$ n.s.

\subsection{6 $F_{1}$-offspring swimming kinematics while feeding in the first week after birth}

Prior to the start of the food treatment (i.e. left of the dashed lines in Fig. 6.6), HF offspring showed a lower mean and maximum speed and acceleration at birth compared to LF offspring (Fig. 6.6a-d). As food availability was not yet manipulated, this indicates a random bias in maternal, and hence offspring, phenotypes at the beginning of the experiment. This difference in swimming kinematics during feeding at birth diminished during the experiment. Maternal food limitation during pregnancy, therefore, had a significant effect on swimming kinematics during feeding. Specifically, the mean and maximum speed and acceleration did not significantly change throughout the experiment in LF offspring $\left(v_{\text {mean }}: \beta_{\text {post.mean }}=-0.2 \times 10^{-3}, P_{\mathrm{MCMC}}=0.948 ; v_{\text {max }}: \beta_{\text {post.mean }}=\right.$ $-1.0 \times 10^{-3}, P_{\mathrm{MCMC}}=0.584 ; a_{\text {mean }}: \beta_{\text {post.mean }}=-1.3 \times 10^{-3}, P_{\mathrm{MCMC}}=0.576 ; a_{\max }$ : $\left.\beta_{\text {post.mean }}=-0.8 \times 10^{-3}, P_{\mathrm{MCMC}}=0.686\right)$, yet significantly increased throughout the experiment in HF offspring $\left(v_{\text {mean }}: \beta_{\text {post.mean }}=0.012, P_{\mathrm{MCMC}}<0.001 ; v_{\max }: \beta_{\text {post.mean }}=\right.$ $0.007, P_{\mathrm{MCMC}}=0.002 ; a_{\text {mean }}: \beta_{\text {post.mean }}=0.008, P_{\mathrm{MCMC}}=0.010 ; a_{\text {max }}: \beta_{\text {post.mean }}=0.005$, $P_{\mathrm{MCMC}}=0.034 ;$ Table S.6.8).

Furthermore, there was a clear ontogenetic effect on the swimming kinematics while feeding during the first week after birth, with two- (Fig. 6.6a-d, middle panels) and seven-dayold (Fig. 6.6a-d, right panels) $\mathrm{F}_{1}$-offspring of both food treatments showing increasingly higher speeds and accelerations than new-born offspring (Fig. 6.6a-d, left panels). This ontogenetic effect was further influenced by maternal food availability during pregnancy, with the locomotor performance of HF offspring improving relative to that of LF offspring. This resulted in significant differences in most, but not all, kinematic parameters between both food treatments in one-week-old offspring born at the end of the experiment $\left(v_{\text {mean }}\right.$ : $\beta_{\text {post.mean }}=1.414, P_{\mathrm{MCMC}}=0.028 ; v_{\max }: \beta_{\text {post.mean }}=1.951, P_{\mathrm{MCMC}}=0.020 ; a_{\text {mean }}$ : $\beta_{\text {post.mean }}=0.340, P_{\mathrm{MCMC}}=0.034 ; a_{\mathrm{max}}: \beta_{\text {post.mean }}=0.646, P_{\mathrm{MCMC}}=0.058 ;$ Fig. $6.6 \mathrm{a}-\mathrm{d}$ right panels). Interestingly, the segregation in locomotor performance between LF and HF offspring throughout the experiment and during the first week after birth was only apparent when food was supplied: during the $5 \mathrm{~min}$ control period (i.e. before food was supplied), LF and HF offspring showed similar mean and maximum speed and acceleration (Table S.6.9; Fig. S.6.2). This suggests that maternal food limitation likely affects 
the feeding capabilities of offspring.

\subsection{7 $F_{2}$-offspring size at birth and growth}

Finally, the effect of maternal food restriction was still noticeable in the $\mathrm{F}_{2}$-generation at birth (Fig. 6.7). Specifically, the cross-sectional body area of $\mathrm{F}_{2}$-offspring at birth was on average $0.16 \mathrm{~mm}^{2}$ smaller in the LF than HF treatment. However, this difference at birth was not significant $\left(P_{\mathrm{MCMC}}=0.228\right)$ and had furthermore completely disappeared in one-week old $\mathrm{F}_{2}$-offspring $\left(\beta_{\text {post.mean }}=-0.008, P_{\mathrm{MCMC}}=0.986\right.$; Fig. 6.7$)$.

Fig. 6.7. $\mathrm{F}_{2}$-offspring body size (i.e. crosssectional body area) $( \pm 95 \% \mathrm{Cl})$ during the first week after birth $(0,2$, or 7 days after birth) estimated in the model described in Table S.6.10 $\left(n_{\mathrm{LF}}=100, n_{\mathrm{HF}}=96\right)$. The growth is predicted for both food treatments (blue: low food; red: high food). Dashed lines represent linear fits throughout the posterior samples of a given food treatment.

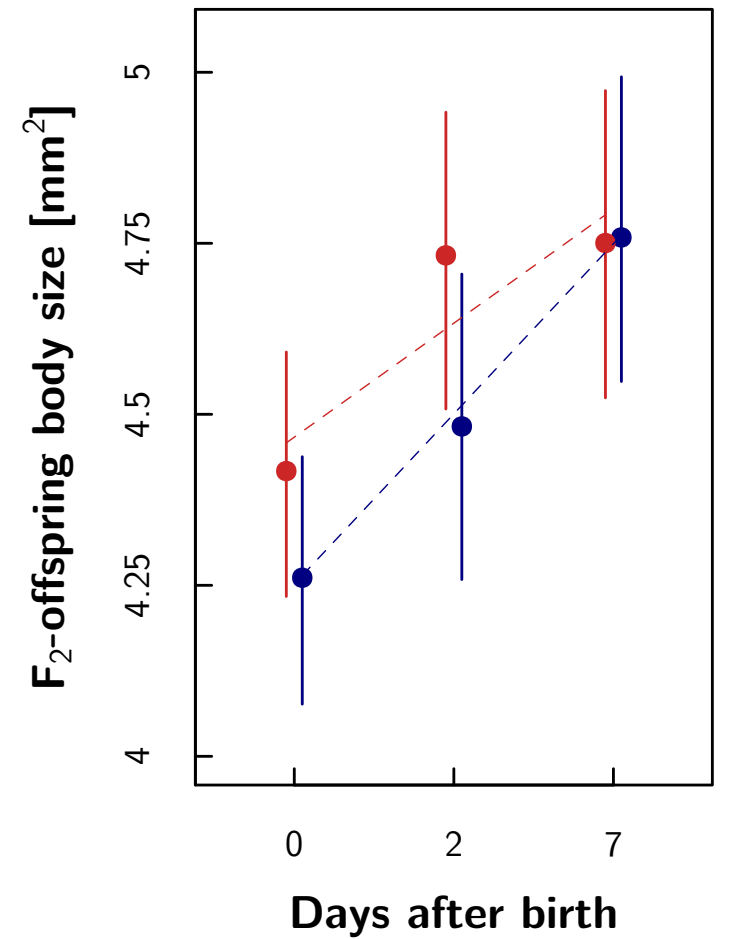

\subsection{Discussion}

We examined to what extent maternal food limitation during pregnancy affects size, quality, and performance of offspring in the matrotrophic live-bearing fish species Phalloptychus januarius. We found that females fed with a 'low-food' (LF) ration during gestation produced significantly smaller offspring at birth compared to females fed with a 'high-food' (HF) ration. The difference in mass persisted during the first week after birth, but had disappeared at adulthood of the offspring. Surprisingly, maternal food limitation during pregnancy did not impact the fast-start escape performance of offspring at birth, however, it did negatively influence several swimming kinematic parameters while feeding during their first week after birth. 


\subsubsection{Effects of food limitation on maternal and offspring phenotype}

Females of both food treatments showed an increase in standard length during the experiment. As expected, LF females tended to lose mass throughout the experiment. Whereas one might expect an increase in mass for HF females fed ad libitum in P. januarius (Pollux \& Reznick, 2011), it did not change. This suggests that both treatments may have been food restricted, albeit differentially, towards the end of the experiment. As food rations remained constant, the HF ration may have caused an unintended mild food restriction. Together with other potential stressors (e.g. individual housing, checking tanks for offspring), this may also explain the slight decline in offspring mass at birth in the HF treatment (Banet \& Reznick, 2008). Despite a smaller difference in food regime than intended, we still see differences in maternal phenotype.

Our food manipulation in $P$. januarius also successfully induced diverging offspring phenotype at birth between both treatments: LF offspring were born lighter and leaner at the end of the experiment compared to HF offspring. As small offspring size at birth in a resource-limited environment is generally considered to be a disadvantage (Parker \& Begon, 1986), matrotrophy is a maladaptive strategy when resources fluctuate unpredictably (Reznick et al., 1996b; Pollux \& Reznick, 2011).

\subsubsection{Short- and long-term compensatory growth mechanisms}

Compensatory growth can be an adaptive strategy to fully or even overcompensate body size in response to increased food availability following a period of growth restriction (Metcalfe \& Monaghan, 2001). In guppies (Poecilia reticulata), juvenile compensatory growth began immediately after a period of growth restriction and continued until maturity (Auer et al., 2010) after which they grew at the same rate as control fish, leading to only partial compensation in mass (Auer et al. 2010; but see Auer 2010). Although LF and HF offspring were fed ad libitum, we observed no compensatory growth for LF offspring in $P$. januarius during the first week after birth. Presumably, the poorer swimming performance during feeding of LF offspring compared to HF offspring, makes it difficult for the smaller LF offspring to catch up in mass during the first week after birth. However, the difference in body mass between both food treatments had disappeared at offspring maturity, indicating that in the long-term LF offspring can fully compensate for their poor nutrition during gestation. This compensatory growth may buffer offspring against long-term fitness consequences of poor nutrition early in life.

Compensatory growth can be achieved by shifting energy investment towards growth at the expense of other functions (Sogard \& Olla, 2002). Such an increased growth rate above 'routine' rate is likely associated with costs. For instance, compensatory growth was shown to negatively affect fecundity in guppies, but not offspring size and quality at birth (Auer et al., 2010). We have not measured fecundity of $\mathrm{F}_{1}$-offspring, but as adults, LF $\mathrm{F}_{1}$-offspring still tended to give birth to smaller $\mathrm{F}_{2}$-offspring compared to $\mathrm{HF} \mathrm{F}_{1}$-offspring. This difference, however, had disappeared in one-week old $\mathrm{F}_{2}$-offspring. It remains to be 
tested whether the reduction in $\mathrm{F}_{2}$-offspring size at birth is due to compensatory growth in the $\mathrm{F}_{1}$-generation or due to a long-term negative consequence of maternal food restriction in the $\mathrm{F}_{0}$-generation that is transmitted via transgenerational phenotypic plasticity.

\subsubsection{Effects of maternal food limitation on offspring fast-start escape performance at birth}

The probability of offspring to respond to a startle stimulus tended to increase over the course of the experiment, for both treatments. Fish may vary their neural threshold for triggering a fast-start response (Wakeling, 2006) depending on stress levels or health condition (Chick \& Van Den Avyle, 2000). Fast escapes are energetically expensive and cannot be repeated at a high rate (Frith, 1990). The observed increase in response rate might thus reflect the modulation of a neuronal threshold, presumably in the Mauthner neurons that mediate the response, or in neurons that stimulate the Mauthner neurons. The increase was, however, observed for both treatment groups and therefore likely results from some change common to both treatments, e.g. both groups probably experienced some degree of food restriction, which may have sufficed to induce the effect.

In studying the fast-start escape behavior, we have at least partially accounted for a change in motivation or threshold by selecting only trials with a clear response to the stimulus. This is important because differences in motivation can introduce noise and variability in the response parameters (Losos et al., 2002). In general, the fast-start escape performance depends on physiological and mechanical muscle properties, as well as muscle activation and body form parameters (Wakeling, 2006; Fleuren et al., 2019). Larger fish typically achieve higher maximum velocities during fast-starts due to larger muscle mass, body length, and production of higher bending moments (Gibb et al., 2006; Wakeling, 2006; Dial et al., 2016; Voesenek et al., 2020). Since LF offspring are born significantly lighter and leaner at the end of the experiment compared to HF offspring, it is surprising that we do not find an effect on their fast-start escape performance. This may be due to a low statistical power to detect significant differences, because our response selection removed about $67 \%$ of the data (Fig. 6.2b). Although additional analyses with different response thresholds indicate that these findings are relatively robust (Supplementary Methods S.6.1.5; Fig. S.6.3-S.6.5), we have to be cautious when concluding that maternal food limitation does not impact fast-start escapes at birth. Future studies should try to maximize sample sizes to yield extended measurements of locomotor performance.

\subsubsection{Effects of maternal food limitation on offspring swimming kinematics during feed- ing}

New-born poeciliid fish are super-precocial, having functional prey-capture abilities at birth and relying on active exogenous feeding after birth (e.g. Girardinus metallicus; Lankheet et al. 2016). Their prey-capturing ability undergoes a rapid integrated development of the visuo-motor system during the first days after birth. Swimming speed 
and acceleration are key parameters determining prey-capture success rate (Lankheet et al., 2016). In P. januarius, these parameters improve rapidly after birth: one-weekold offspring showed higher mean and maximum speeds and accelerations than new-born offspring. This postnatal improvement of locomotor performance was present in both the absence and presence of food, and is thus likely the direct result of an ontogenetic development of external body morphology, as well as maturation of internal physiology (Gibb et al., 2006).

We further found that the $\mathrm{F}_{1}$-offspring swimming kinematics during feeding develop differently after birth depending on maternal food availability during pregnancy. The locomotor performance during feeding improved significantly faster in HF offspring compared to that of LF offspring, during the first week after birth. This difference cannot be explained by differences in ontogenetic growth between HF and LF offspring: although HF offspring were larger at birth than LF offspring, they showed similar increases in size during the first week after birth. Moreover, the segregation in locomotor performance between LF and HF offspring was only apparent when food was supplied, and thus, may at least partly be related to differently developing feeding capabilities after birth.

Rather than only being born smaller, LF offspring also tended to have less body fat at birth and may have differed in other morphological and physiological features that can influence the postnatal development of locomotor performance during feeding. For instance, smaller guppy offspring were shown to have a lower degree of skeletal ossification at birth, which is considered a proxy for internal maturity (Dial et al., 2016). Skeletal ossification can directly affect swimming performance (Dial et al., 2016), and may also influence postnatal development of locomotor performance. In addition, locomotor performance depends on muscle fiber type (Rome et al., 1988) that undergoes a distinct shift in composition after birth (Veggetti et al., 1993). Similarly, maximum body curvature during prey-capture was shown to change after birth (Lankheet et al., 2016). Particularly, an increase in muscle mass-specific power output induces a higher body curvature (Wakeling, 2006), and consequently improves prey-capture success rate if the motion control is sufficiently matured (Lankheet et al., 2016). Better prey-capturing success enables uptake of more energy (via feeding) per unit of time, which can be allocated to either growth or quality (e.g. body fat). It is thus possible that LF and HF offspring differ in various morphological or behavioral parameters at birth (e.g. degree of skeletal ossification, composition of muscle fiber type, or body curvature) that, furthermore, differently develop between both food treatments after birth. The improved prey-capture success and increased efficiency of resource acquisition in HF offspring compared to LF offspring may also explain why HF offspring gained body fat during the first week after birth, but LF offspring did not.

Overall, our findings suggest that differential resource allocation of mothers to offspring may not only cause LF offspring to be born smaller than HF offspring, but also 'worseconditioned', which consequently is likely to negatively impact the postnatal maturation of tissues associated with locomotion, and hence feeding efficiency, body condition, and 
likely fitness during early life. Despite ad libitum feeding after birth, LF offspring fall further behind rather than compensating for their smaller size at birth. These initial differences however disappear towards adulthood.

\subsection{Conclusion}

Our study suggests that matrotrophy in poeciliids is likely a maladaptive strategy in fluctuating resource environments, because sudden reductions in maternal food availability during pregnancy result in smaller offspring with a lower locomotor performance during early life. Future studies should try to explicitly quantify the consequences of maternal food limitation on prey-capture success rate of offspring to better understand the role of postnatal maturation of tissues associated with locomotion in shaping offspring growth and hence fitness.

\section{S.6 Supplementary Information}

\section{S.6.1 Supplementary Methods}

\section{S.6.1.1 Measurements of dry mass and body fat}

Dry mass and body fat was measured after euthanizing the fish with an overdose of MS222 and preserving in $4 \%$ formaldehyde. Dry mass was measured to the nearest $0.01 \mathrm{mg}$ on a Mettler Toledo AE163 Microbalance (Mettler Instruments Corp., Hightstown, New Jersey, USA) after air-drying overnight at $60^{\circ} \mathrm{C}$ in a drying oven. Lean mass was measured by extracting the fat twice with anhydrous diethyl ether to remove triglycerides, and by subsequently air-drying and re-weighting the fish. The proportion of body fat was then calculated by subtracting lean mass from dry mass divided by dry mass.

\section{S.6.1.2 Image analysis}

Images were recorded at $75 \mathrm{~Hz}$ (feeding experiments) or $350 \mathrm{~Hz}$ (fast-start experiments) with exposure times of $600 \mu \mathrm{s}$ and at a spatial resolution of $5 \mathrm{pix} \cdot \mathrm{mm}^{-1}$. The sensitivity of the camera (Basler Ace 2040 90um NIR USB3) was increased by a factor of four, by binning pixels both horizontally and vertically by a factor of two. This reduced image size to $1024 \times 1024$ pixels. Camera images were analyzed on the fly while conducting the experiments, using Python and OpenCV. Noise was removed from images by applying a median filter (3 pixels). After subtraction of a background image (without fish present) we applied a fixed threshold to detect the silhouette of the fish. Difference images were again subjected to a median filter (3 pixels) to remove noise. Fish were then distinguished from false hits by applying lower and upper limits to the size of the object, and lower and upper limits to the length-width ratio. We used Kalman filters to track the individual fish and estimate their path of motion, based on the location of the center of mass of 
the fish silhouette. To accelerate the analyses and thus to be able to track multiple fish simultaneously at high frame rates, we alternated between full analysis of the total image, as described above, and analyzing only those parts in which fish were previously detected. For the latter we used the Kalman prediction to select an area of $200 \times 200$ pixels centered on the predicted, new location. For each frame and each fish detected we saved the location estimated by the Kalman filter, together with the full contour of the silhouette. Exact timing of events defining control and feeding intervals, as well as the timing of the startle stimulus were also automatically saved to file.

\section{S.6.1.3 $\quad \mathrm{F}_{2}$-offspring body size}

Due to ethical restrictions, the dry mass and body fat of $\mathrm{F}_{2}$-offspring could not be measured. Instead, body size of $\mathrm{F}_{2}$-offspring was quantified as the average cross-sectional body area measured from the dorsal side in live animals in the experimental set-up used to record the swimming kinematics. A maximum of nine Petri dishes with each containing a single fish were simultaneously placed on pre-defined positions on the translucent plate (arranged in a $3 \times 3$ pattern) and filmed from the dorsal side with the high-speed camera (Fig. 6.2). For each frame, we subsequently calculated the surface area of the fish based on its silhouette (see previous section). The cross-sectional body area was then defined as the average surface area among all frames.

\section{S.6.1.4 Local polynomial regression smoothing}

We used an in-house developed Python program to track the fish's silhouette in realtime and to automatically extract the location (i.e. X- and Y-position) of the center of mass through time. Swimming speed was calculated by numerically differentiating the fish's position, and linear acceleration was calculated by differentiating the resulting swimming speed. We performed local polynomial regression smoothing as a function of frame number before each differentiation step using the function loess in R v 3.5 (R Core Team, 2020). This procedure removed high-frequency noise in the data. The order of the polynomial was 2 , and the proportion of the data that was considered to be neighbouring $x$ (i.e. the degree of smoothing, $\alpha$ ) was 0.015 for the swimming kinematics during feeding, and ranged between 0.025 and 0.12 for the fast-start escape responses. The reason for a varying $\alpha$ in the latter case was a larger variation in the number of frames (i.e. available data) among individual fast-starts. Rather than having a constant $\alpha$ for all fast-starts, we therefore calculated $\alpha$ for each individual fast-start as:

$$
\alpha_{i}=\frac{0.025 \cdot \mathrm{F}_{\max }}{n_{i}},
$$

where $\mathrm{F}_{\max }$ is the maximum number of frames recorded during the fast-starts, and $n_{i}$ the available number of frames during the $i$ th fast-start. 


\section{S.6.1.5 Defining the response threshold during the fast-start escape response}

When plotting a histogram of all instant fast-start speeds observed in the light red rectangle shown in Figure $6.2 \mathrm{~b}$ (i.e. the period from which the locomotor performance parameters were extracted), the histogram shows two peaks (Fig. S.6.3). These correspond to individuals that have either responded to the stimulus (i.e. by dropping a weight on the plate) or not. We considered fish as having 'responded' to the stimulus if their speed during a narrow time window around the maximum of the overall mean speed ( \pm 3 frames) exceeded $60 \mathrm{~mm} \cdot \mathrm{s}^{-1}$. This response threshold was defined as the speed observed at the minimum frequency in between the two peaks of the histogram (Fig. S.6.3).

The potential effects of maternal food limitation on offspring performance during the faststart escape response were then analyzed by fitting the mean and maximum speed and acceleration in a multivariate LMM. Here, only individuals that have 'responded' to the stimulus were included in the model. Fixed effects included treatment (low food or high food), experimental day, as well as treatment $\times$ day.

Defining a response threshold is important because differences in motivation can introduce noise and variability in the response parameters (Losos et al., 2002). However, it can also be biased, as the sample size depends on the threshold: the larger the value, the more fish are considered to have not responded to the stimulus, and thus, more data is removed.

To quantify the sensitivity of our results to different thresholds, we defined five additional threshold values in between the two peaks of the histogram $(d=20,40,80,100$, and 120 $\mathrm{mm} \cdot \mathrm{s}^{-1}$; Fig. S.6.3). For each $d$, we subsequently extracted the corresponding locomotor performance parameters (i.e. mean and maximum speed and acceleration) and applied the LMM (as described above) to the resulting data sets. To compare the strength of individual relationships, the slopes $(\beta)$ were standardized by multiplying with the phenotypic standard deviation of the predictor variable (i.e. experimental day) and dividing by the phenotypic standard deviation of the response variable (i.e. mean and maximum speed and acceleration) (Schielzeth, 2010). The resulting effect sizes $\left(\beta^{*}\right)$ take values between -1 and 1.

The results show that the interaction between treatment and experimental day is not significant for all threshold values and locomotor performance traits. Moreover, the slopes are relatively robust to threshold values between 40 and $100 \mathrm{~mm} \cdot \mathrm{s}^{-1}$ (Fig. S.6.4, S.6.5). The effect sizes (i.e. standardized slopes) of experimental day on the locomotor performance parameters show a relatively small range for the low food $\left(v_{\text {mean }}=-0.03-0.10\right.$; $\left.v_{\max }=-0.12-0.02 ; a_{\text {mean }}=-0.08--0.02 ; a_{\max }=-0.28--0.19\right)$ and high food treatment $\left(v_{\text {mean }}=-0.02-0.19 ; v_{\max }=-0.03-0.24 ; a_{\text {mean }}=-0.20--0.08 ; a_{\max }=-0.22--0.04\right)$. By contrast, the output is relatively sensitive to threshold values closer to the two peaks of the histogram (i.e. $d=20$ and 120) (Fig. S.6.4). This is not surprising, as a low threshold causes more noise and variability in the response parameters. The opposite is true for a high threshold. A threshold of $d=120$ includes much less noise, but also removes more data $\left(d_{120}=73 \%\right.$ versus $d_{20}=60 \%$ data loss). 
To balance the conflict between maximizing sample size and minimizing noise, we therefore chose a threshold in the middle of two peaks of the histogram. Specifically, we defined the threshold at $60 \mathrm{~mm} \cdot \mathrm{s}^{-1}$, which is the speed observed at the minimum frequency in between the two peaks of the histogram.

\section{S.6.2 Supplementary Results}

Fig. S.6.1. Number of offspring produced by a female (i.e. maternal fecundity) $( \pm 95 \% \mathrm{Cl})$ within a given experimental week estimated in the model described in Table S.6.3 $\left(n_{\mathrm{LF}}=\right.$ $\left.175, n_{\mathrm{HF}}=175\right)$. blue: low food; red: high food. Dashed vertical line corresponds to the start of the food treatment. $P_{\mathrm{MCMC}}$-value for the interaction between experimental week and treatment is given at the top.

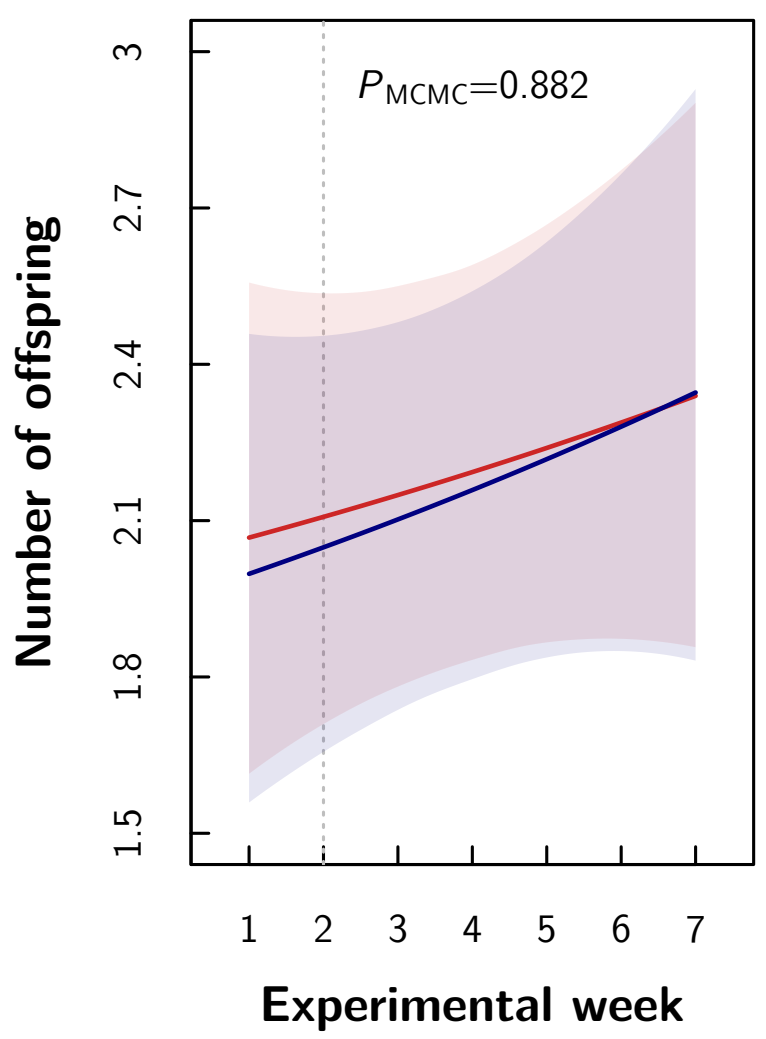




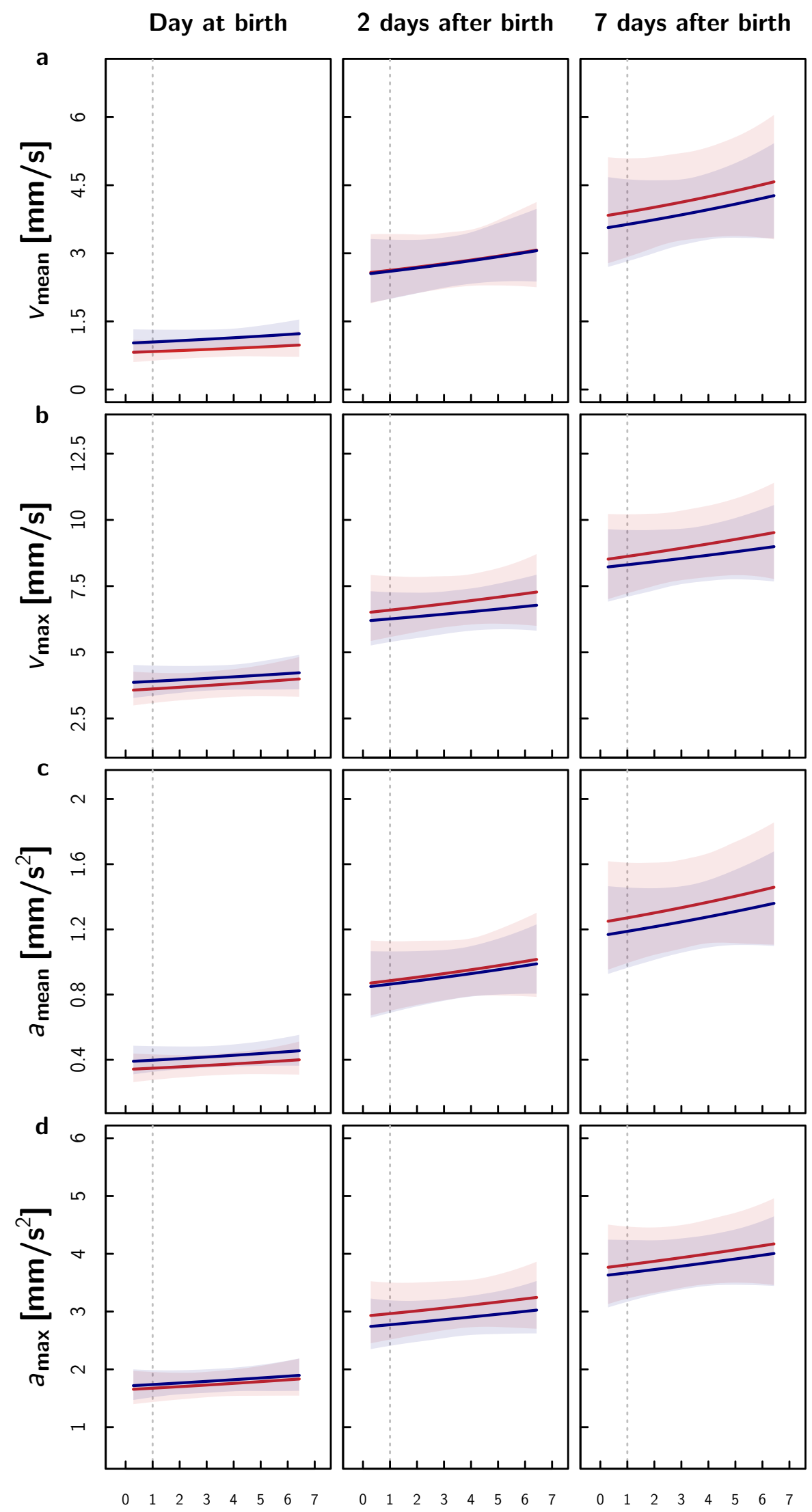

Experimental weeks Experimental weeks Experimental weeks 
Fig. S.6.2. Swimming kinematics during feeding trials without food supply (i.e. control) estimated in the model described in Table S.6.9 $\left(n_{\mathrm{LF}}=190, n_{\mathrm{HF}}=144\right)$. New-borns were collected weekly to assess swimming kinematics throughout the 7-week maternal food treatment (see Fig. 6.1b,c). a Mean speed, b maximum speed, c mean acceleration, and d maximum acceleration $( \pm 95 \%$ $\mathrm{Cl}$ ) are predicted throughout the experiment for both food treatments (blue: low food; red: high food), as well as a function of age (left panels: 0 days old; middle panels: 2 days old; right panels: 7 days old). Dashed vertical line corresponds to the start of the food treatment. Significance codes are given at the top for the difference between both food treatments at the start of the food treatment and at the end of the experiment. Significant codes: $P_{\mathrm{MCMC}} \leq 0.001^{* * *},<0.01^{* *}$, $\leq 0.05^{*},>0.05$ n.s.

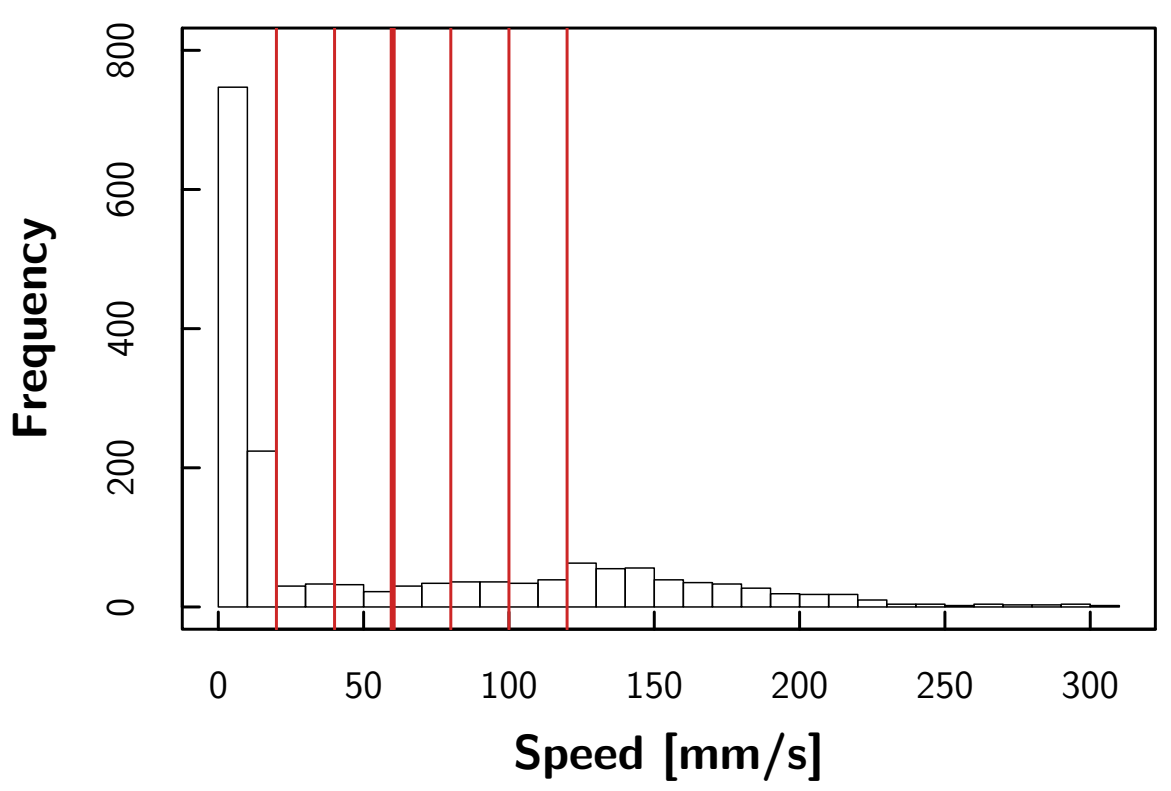

Fig. S.6.3. Histogram of all instant fast-start speeds observed in the light red rectangle shown in Figure 6.2b. The histogram shows two peaks, which correspond to individuals that have either responded to the stimulus or not. To quantify the sensitivity of our results to different response thresholds, we defined six threshold values in between the two peaks of the histogram $(d=20,40$, $60,80,100$, and $120 \mathrm{~mm} \cdot \mathrm{s}^{-1}$; red vertical lines). We finally defined the threshold at $60 \mathrm{~mm} \cdot \mathrm{s}^{-1}$ (thick red vertical line), which is the speed observed at the minimum frequency in between the two peaks of the histogram. 

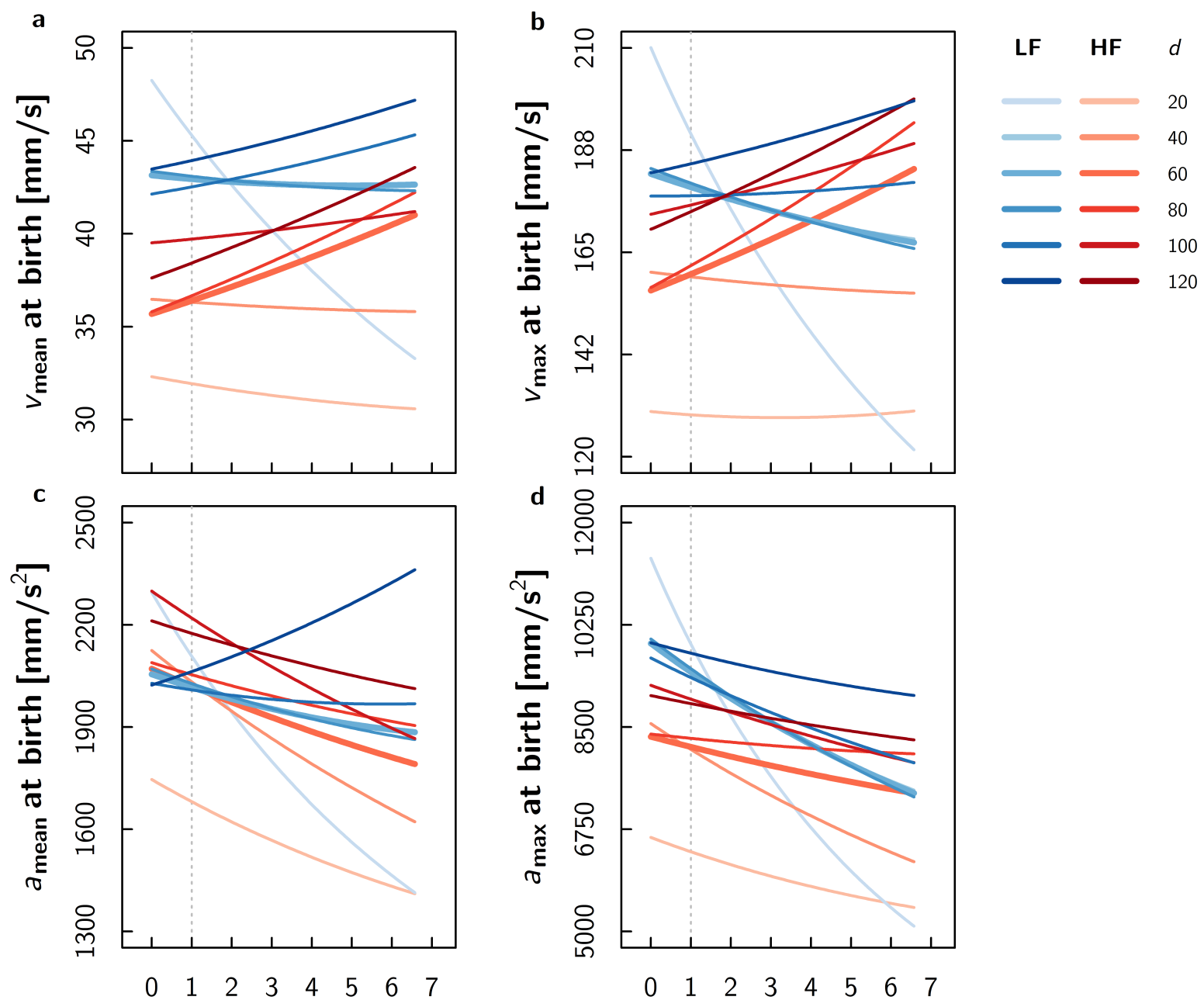

Experimental weeks

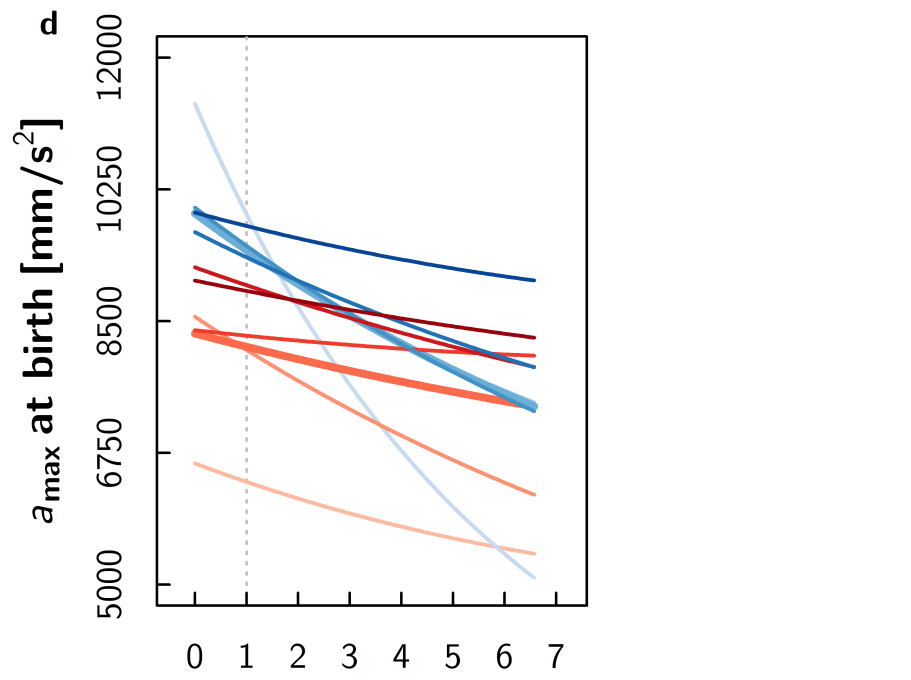

Experimental weeks

Fig. S.6.4. a-d Swimming performance of new-born offspring during the fast-start escape response depending on different response thresholds $\left(d=20,40,60,80,100\right.$, and $\left.120 \mathrm{~mm} \cdot \mathrm{s}^{-1}\right)$. Newborns were collected weekly to assess the fast-start escape response throughout the 7-week maternal food treatment (see Fig. 6.1b). a Mean speed, b maximum speed, c mean acceleration, and $\mathbf{d}$ maximum acceleration at birth are predicted for both food treatments (blue: low food; red: high food) and throughout the experiment. Vertical dotted grey line corresponds to the start of the food treatment. We finally defined the threshold at $60 \mathrm{~mm} \cdot \mathrm{s}^{-1}$ (thicker lines), which is the speed observed at the minimum frequency in between the two peaks of the histogram shown in Figure S.6.3. 


\section{$v_{\text {mean }}$ at birth $[\mathrm{mm} / \mathrm{s}] \quad v_{\max }$ at birth $[\mathrm{mm} / \mathrm{s}]$}
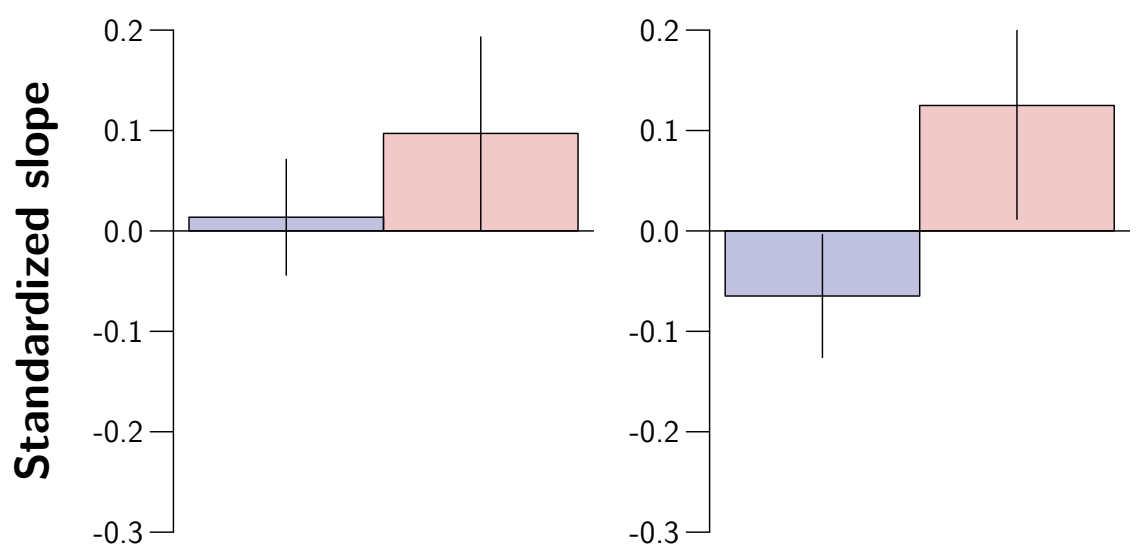

$a_{\text {mean }}$ at birth $\left[\mathrm{mm} / \mathrm{s}^{2}\right] \quad a_{\max }$ at birth $\left[\mathrm{mm} / \mathrm{s}^{2}\right]$
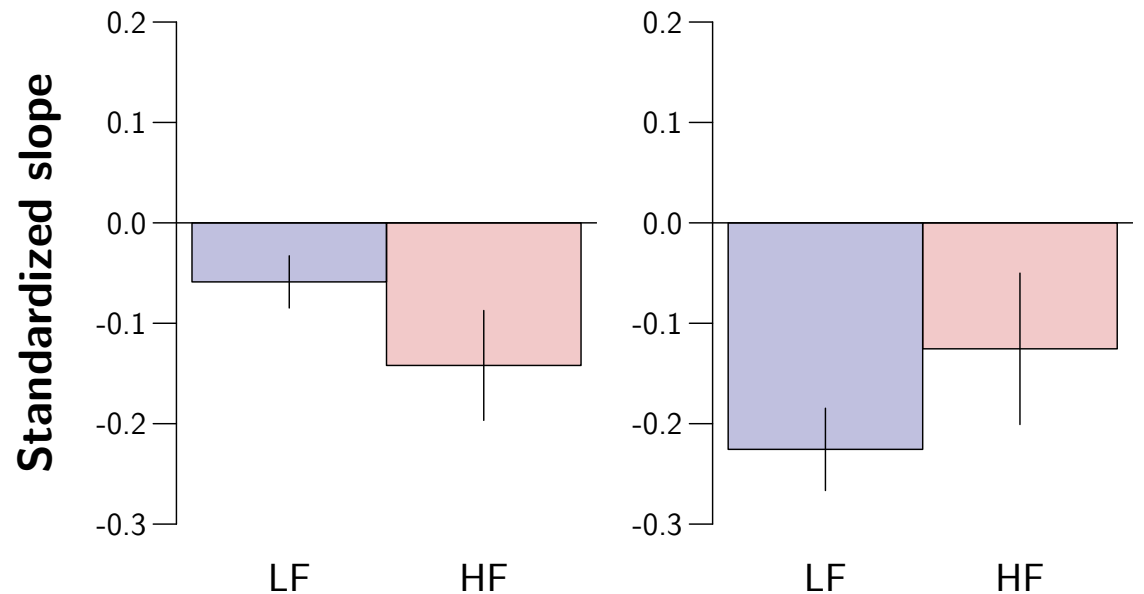

Fig. S.6.5. Standardized effect size ( \pm sd) of experimental day on the locomotor performance parameters (i.e. mean and maximum speed and acceleration) during the fast-start escape responses. The effect sizes are averaged over the results of four different response thresholds $(d=40,60$, 80 , and $100 \mathrm{~mm} \cdot \mathrm{s}^{-1}$, i.e. excluding the two extremes $d=20$ and $120 \mathrm{~mm} \cdot \mathrm{s}^{-1}$ ) and plotted as a function of treatment (blue: low food; red: high food). The effect sizes take values between -1 and 1 . 
Table S.6.1. Fixed and random effects in explaining variation in offspring survival (i.e. whether offspring was found dead or alive) estimated in a generalized linear mixed effects model using the MCMCglmm package (Hadfield, 2010). A logit link was used for the Bernoulli-distributed response. The default normal priors were used for the fixed effects with the expected value of 0 and variance $10^{12}$. Inverse-Wishart priors were used for the variances with the expected value of 1 and degree of belief of 0.002 . As with binary models the residual variance is not identified, it was fixed at 1 . The number of iterations was $1,500,000$, with a burnin of 500,000 and a thinning of 1000 .

\begin{tabular}{|c|c|c|c|c|c|c|}
\hline \multicolumn{7}{|c|}{ Offspring survival } \\
\hline \multicolumn{7}{|c|}{ Fixed effects } \\
\hline & $\beta_{\text {post.mean }}$ & $2.5 \% \mathrm{Cl}$ & $97.5 \% \mathrm{Cl}$ & eff.samp & $P_{\mathrm{MCMC}}$ & Sig. \\
\hline intercept & 3.396 & 2.148 & 4.637 & 1000.000 & 0.001 & $* * *$ \\
\hline LF treatment & 0.174 & -1.691 & 1.874 & 1000.000 & 0.830 & n.s. \\
\hline experimental day & -0.065 & -0.100 & -0.034 & 1000.000 & 0.001 & $* * *$ \\
\hline LF treatment $\times$ experimental day & 0.008 & -0.035 & 0.063 & 1000.000 & 0.732 & n.s. \\
\hline
\end{tabular}

\begin{tabular}{ccccc}
\hline \multicolumn{5}{c}{ Random effects } \\
\hline & $\sigma_{\text {post.mean }}^{2}$ & $2.5 \% \mathrm{Cl}$ & $97.5 \% \mathrm{Cl}$ & eff.samp \\
\hline mother identity & 3.1769 & 1.1863 & 5.6963 & 1000.0000 \\
\hline
\end{tabular}

$\beta_{\text {post.mean }}$ : posterior mean of regression coefficient; $\mathrm{Cl}$ : confidence interval; eff.samp: effective sample size; $P_{\mathrm{MCMC}}$ : posterior bayesian $p$-value; LF: low food; significant codes: $P_{\mathrm{MCMC}} \leq 0.001^{* * *},<0.01^{* *}, \leq 0.05^{*},>0.05$ n.s. 
Table S.6.2. Fixed and random effects in explaining variation in maternal (a) wet mass ( $\mathrm{g}$ ) and (b) standard length $(\mathrm{mm})$ estimated using the MCMCglmm package (Hadfield, 2010). Both traits were fitted in a bivariate linear mixed effects model allowing for the covariance between the residuals of both responses. The default normal priors were used for the fixed effects with the expected value of 0 and variance $10^{12}$. Inverse-Wishart priors were used for the variances with the expected value of $1 \cdot 10^{-6}$ and degree of belief of $k+1$, where $k$ is the dimension of the covariance matrix. The number of iterations was 5,500,000, with a burnin of 500,000 and a thinning of 5000 .

\section{(a) Maternal wet mass}

\begin{tabular}{rcccccc}
\hline \multicolumn{7}{c}{ Fixed effects } \\
\hline & $\beta_{\text {post.mean }}$ & $2.5 \% \mathrm{Cl}$ & $97.5 \% \mathrm{Cl}$ & eff.samp & $P_{\mathrm{MCMC}}$ & Sig. \\
intercept & 0.457 & 0.420 & 0.500 & 1000.000 & 0.001 & $* * *$ \\
LF treatment & -0.003 & -0.063 & 0.056 & 1000.000 & 0.918 & n.s. \\
experimental day & -0.011 & -0.069 & 0.046 & 1000.000 & 0.732 & n.s. \\
LF $\times$ experimental day 51 & -0.042 & -0.128 & 0.045 & 1000.000 & 0.344 & n.s. \\
\hline
\end{tabular}

\begin{tabular}{lcccc}
\hline \multicolumn{5}{c}{ Random effects } \\
\hline & $\sigma_{\text {post.mean }}^{2}$ & $2.5 \% \mathrm{Cl}$ & $97.5 \% \mathrm{Cl}$ & eff.samp \\
\hline mother identity & $0.0041 \times 10^{-3}$ & $0.0002 \times 10^{-3}$ & $0.0089 \times 10^{-3}$ & 1000.0000 \\
$\theta_{\text {wet mass, wet mass }}$ & 0.0112 & 0.0083 & 0.0145 & 1000.0000 \\
$\theta_{\text {wet mass,standard length }}$ & 0.1939 & 0.1390 & 0.2525 & 1000.0000 \\
\hline
\end{tabular}

(b) Maternal standard length

\begin{tabular}{rcccccc}
\hline \multicolumn{10}{c}{ Fixed effects } \\
\hline intercept & 26.847 & 26.098 & 27.601 & 1000.000 & 0.001 & $* * *$ \\
LF treatment & -0.630 & -1.709 & 0.402 & 1000.000 & 0.270 & n.s. \\
experimental day 51 & 0.427 & -0.594 & 1.466 & 1000.000 & 0.456 & n.s. \\
LF treatment xexperimental day 51 & 0.046 & -1.487 & 1.701 & 1000.000 & 0.958 & n.s. \\
\hline
\end{tabular}

\begin{tabular}{lcccc}
\hline \multicolumn{5}{c}{ Random effects } \\
\hline & $\sigma_{\text {post.mean }}^{2}$ & $2.5 \% \mathrm{Cl}$ & $97.5 \% \mathrm{Cl}$ & eff.samp \\
\hline mother identity & $0.0032 \times 10^{-3}$ & $0.0002 \times 10^{-3}$ & $0.0082 \times 10^{-3}$ & 1000.0000 \\
$\theta_{\text {standard length,standard length }}$ & 3.9308 & 2.8323 & 5.0524 & 1000.0000 \\
$\theta_{\text {wet mass,standard length }}$ & 0.1939 & 0.1390 & 0.2525 & 1000.0000 \\
\hline
\end{tabular}

$\beta_{\text {post.mean: }}$ posterior mean of regression coefficient; $\mathrm{Cl}$ : confidence interval; eff.samp: effective sample size; $P_{\mathrm{MCMC}}$ : posterior bayesian $p$-value; LF: low food; $\theta$ : residual (co)variance between two responses; significant codes: $P_{\mathrm{MCMC}} \leq$ $0.001^{* * *},<0.01^{* *}, \leq 0.05^{*},>0.05$ n.s. 
Table S.6.3. Fixed and random effects in explaining variation in maternal fecundity (i.e. number of embryos) estimated in a generalized linear mixed effects model using the MCMCglmm package (Hadfield, 2010). A log link was used for the Poisson-distributed response. The default normal priors were used for the fixed effects with the expected value of 0 and variance $10^{12}$. InverseWishart priors were used for the variances with the expected value of 1 and degree of belief of 0.002 . The number of iterations was $1,500,000$, with a burnin of 500,000 and a thinning of 1000 .

\begin{tabular}{rcccccc}
\hline \multicolumn{10}{c}{ Maternal fecundity } \\
\hline \multicolumn{7}{c}{ Fixed effects } \\
intercept & 0.699 & 0.398 & 0.932 & 893.084 & 0.001 & $* * *$ \\
LF treatment & -0.040 & -0.425 & 0.318 & 827.383 & 0.818 & n.s. \\
experimental week & 0.021 & -0.029 & 0.069 & 802.007 & 0.374 & n.s. \\
LF treatment $\times$ experimental week & 0.006 & -0.059 & 0.078 & 844.761 & 0.882 & n.s. \\
\hline
\end{tabular}

\begin{tabular}{lcccc}
\hline \multicolumn{5}{c}{ Random effects } \\
\hline & $\sigma_{\text {post.mean }}^{2}$ & $2.5 \% \mathrm{Cl}$ & $97.5 \% \mathrm{Cl}$ & eff.samp \\
\hline mother identity & 0.1210 & 0.0518 & 0.2109 & 607.1644 \\
residual & 0.0058 & 0.0002 & 0.0187 & 696.9852 \\
\hline
\end{tabular}

$\beta_{\text {post.mean }}$ posterior mean of regression coefficient; $\mathrm{Cl}$ : confidence interval; eff.samp: effective sample size; $P_{\text {MCMC }}$ : posterior bayesian $p$-value; LF: low food; significant codes: $P_{\mathrm{MCMC}} \leq 0.001^{* * *},<0.01^{* *}, \leq 0.05^{*}$, $>0.05$ n.s. 
Table S.6.4. Fixed and random effects in explaining variation in $F_{1}$-offspring (a) dry mass ( $\mathrm{mg}$ ), (b) lean mass (mg), and (c) square-root transformed body fat estimated using the MCMCglmm package (Hadfield, 2010). All traits were fitted in a multivariate linear mixed effects model allowing for the covariance between the residuals of all responses. The default normal priors were used for the fixed effects with the expected value of 0 and variance $10^{12}$. Inverse-Wishart priors were used for the variances with the expected value of $1 \cdot 10^{-6}$ and degree of belief of $k+1$, where $k$ is the dimension of the covariance matrix. The number of iterations was 5,500,000, with a burnin of 500,000 and a thinning of 5000 .

\begin{tabular}{rcccccc}
\hline \multicolumn{7}{c}{ (a) F1-offspring dry mass } \\
\hline & $\beta_{\text {post.mean }}$ & $2.5 \% \mathrm{Cl}$ & $97.5 \% \mathrm{Cl}$ & eff.samp & $P_{\mathrm{MCMC}}$ & Sig. \\
intercept & 1.139 & 1.051 & 1.239 & 1097.891 & 0.001 & $* * *$ \\
LF treatment & 0.103 & 0.003 & 0.199 & 1000.000 & 0.038 & $*$ \\
experimental day & -0.003 & -0.006 & -0.001 & 1000.000 & 0.012 & $*$ \\
day 7 after birth & 0.340 & 0.238 & 0.425 & 1105.901 & 0.001 & $* * *$ \\
experimental day & -0.026 & -0.052 & -0.000 & 1000.000 & 0.048 & $*$ \\
alive & 0.270 & 0.211 & 0.335 & 1000.000 & 0.001 & $* * *$ \\
LF treatment $\times$ experimental day & -0.007 & -0.010 & -0.003 & 1000.000 & 0.001 & $* * *$ \\
LF treatment $\times$ day 7 after birth & -0.071 & -0.182 & 0.059 & 1000.000 & 0.264 & n.s. \\
\hline
\end{tabular}

\begin{tabular}{lcccc}
\hline \multicolumn{5}{c}{ Random effects } \\
\hline & $\sigma_{\text {post.mean }}^{2}$ & $2.5 \% \mathrm{Cl}$ & $97.5 \% \mathrm{Cl}$ & eff.samp \\
\hline mother identity & $0.0032 \times 10^{-3}$ & $0.0002 \times 10^{-3}$ & $0.0131 \times 10^{-3}$ & 1118.8416 \\
$\theta_{\text {dry mass,dry mass }}$ & 0.0888 & 0.0794 & 0.0990 & 1000.0000 \\
$\theta_{\text {dry mass,lean mass }}$ & 0.0733 & 0.0655 & 0.0826 & 1000.0000 \\
$\theta_{\text {dry mass,body fat }}$ & 0.0203 & 0.0168 & 0.0234 & 959.0282 \\
\hline
\end{tabular}




\begin{tabular}{|c|c|c|c|c|c|c|c|c|}
\hline \multicolumn{9}{|c|}{ (b) $F_{1}$-offspring lean mass } \\
\hline \multicolumn{9}{|c|}{ Fixed effects } \\
\hline \multirow{2}{*}{\multicolumn{2}{|c|}{ intercept }} & $\beta_{\text {post.mean }}$ & $2.5 \% \mathrm{Cl}$ & $97.5 \% \mathrm{Cl}$ & \multicolumn{2}{|c|}{ eff.samp } & $P_{\mathrm{MCMC}}$ & Sig. \\
\hline & & 0.965 & 0.889 & 1.050 & \multicolumn{2}{|c|}{1000.000} & 0.001 & $* * *$ \\
\hline \multicolumn{2}{|c|}{ LF treatment } & 0.091 & -0.000 & 0.169 & \multicolumn{2}{|c|}{1000.000} & 0.032 & $*$ \\
\hline \multicolumn{2}{|c|}{ experimental day } & -0.002 & -0.004 & -0.000 & \multicolumn{2}{|c|}{1000.000} & 0.038 & $*$ \\
\hline \multicolumn{2}{|c|}{ day 7 after birth } & 0.328 & 0.246 & 0.402 & \multicolumn{2}{|c|}{1114.867} & 0.001 & $* * *$ \\
\hline \multicolumn{2}{|c|}{ experimental day ${ }^{2}$} & -0.022 & -0.044 & 0.000 & \multicolumn{2}{|c|}{1000.000} & 0.042 & $*$ \\
\hline \multicolumn{2}{|c|}{ alive } & 0.245 & 0.186 & 0.292 & \multicolumn{2}{|c|}{1000.000} & 0.001 & $* * *$ \\
\hline \multirow{2}{*}{\multicolumn{2}{|c|}{$\begin{array}{l}\text { LF treatment } \times \text { experimental day } \\
\text { LF treatment } \times \text { day } 7 \text { after birth }\end{array}$}} & -0.006 & -0.009 & -0.003 & \multicolumn{2}{|c|}{1000.000} & 0.001 & $* * *$ \\
\hline & & -0.052 & -0.158 & 0.053 & \multicolumn{2}{|c|}{1000.000} & 0.330 & n.s. \\
\hline \multicolumn{9}{|c|}{ Random effects } \\
\hline & \multicolumn{2}{|c|}{$\sigma_{\text {post.mean }}^{2}$} & $2.5 \% \mathrm{Cl}$ & \multicolumn{2}{|c|}{$97.5 \% \mathrm{Cl}$} & \multicolumn{2}{|c|}{ eff.samp } & \\
\hline mother identity & \multicolumn{2}{|c|}{$0.0044 \times 10^{-3}$} & $0.0002 \times 10^{-3}$ & \multicolumn{2}{|c|}{$0.0186 \times 10^{-3}$} & \multicolumn{2}{|c|}{1000.0000} & \\
\hline$\theta_{\text {lean mass,lean mass }}$ & \multicolumn{2}{|c|}{0.0650} & 0.0578 & \multicolumn{2}{|c|}{0.0730} & \multicolumn{2}{|c|}{1000.0000} & \\
\hline$\theta_{\text {lean mass,dry mass }}$ & \multicolumn{2}{|c|}{0.0733} & 0.0655 & \multicolumn{2}{|c|}{0.0826} & \multicolumn{2}{|c|}{1000.0000} & \\
\hline$\theta_{\text {lean mass,body fat }}$ & \multicolumn{2}{|c|}{0.0108} & 0.0082 & \multicolumn{2}{|c|}{0.0132} & \multicolumn{2}{|c|}{1107.0572} & \\
\hline
\end{tabular}

\begin{tabular}{rcccccc}
\hline \multicolumn{7}{c}{ (c) $\mathbf{F}_{1}$-offspring body fat } \\
\hline \multicolumn{7}{c}{ Fixed effects } \\
intercept & 0.402 & 0.364 & 0.440 & 1000.000 & 0.001 & $* * *$ \\
LF treatment & 0.019 & -0.018 & 0.058 & 1000.000 & 0.356 & n.s. \\
experimental day & -0.001 & -0.002 & -0.000 & 1000.000 & 0.010 & $* *$ \\
day 7 after birth & 0.019 & -0.018 & 0.058 & 1000.000 & 0.322 & n.s. \\
experimental day & -0.006 & -0.016 & 0.004 & 1000.000 & 0.218 & n.s. \\
alive & 0.034 & 0.008 & 0.059 & 1000.000 & 0.006 & $* *$ \\
LF treatment $\times$ experimental day & -0.001 & -0.002 & 0.000 & 1000.000 & 0.110 & n.s. \\
LF treatment $\times$ day 7 after birth & -0.022 & -0.070 & 0.028 & 1000.000 & 0.378 & n.s. \\
\hline
\end{tabular}

\begin{tabular}{ccccc}
\hline \multicolumn{5}{c}{ Random effects } \\
\hline & $\sigma_{\text {post.mean }}^{2}$ & $2.5 \% \mathrm{Cl}$ & $97.5 \% \mathrm{Cl}$ & eff.samp \\
\hline mother identity & $0.0019 \times 10^{-3}$ & $0.0002 \times 10^{-3}$ & $0.0054 \times 10^{-3}$ & 718.8426 \\
$\theta_{\text {body fat,body fat }}$ & 0.0136 & 0.0120 & 0.0154 & 841.9908 \\
$\theta_{\text {body fat,dry mass }}$ & 0.0203 & 0.0168 & 0.0234 & 959.0282 \\
$\theta_{\text {body fat,lean mass }}$ & 0.0108 & 0.0082 & 0.0132 & 1107.0572 \\
\hline
\end{tabular}

$\beta_{\text {post.mean }}$ : posterior mean of regression coefficient; $\mathrm{Cl}$ : confidence interval; eff.samp: effective sample size; $P_{\mathrm{MCMC}}$ : posterior bayesian $p$-value; LF: low food; $\theta$ : residual (co)variance between two responses; significant codes: $P_{\mathrm{MCMC}} \leq 0.001^{* * *},<0.01^{* *}, \leq 0.05^{*},>0.05$ n.s. 
Table S.6.5. Fixed effects in explaining variation in $\mathrm{F}_{1}$-adult (a) dry mass ( $\mathrm{g}$ ), (b) lean mass (g), and (c) square-root transformed body fat estimated using the MCMCglmm package (Hadfield, 2010). All traits were fitted in a multivariate linear model allowing for the covariance between the residuals of all responses. The default normal priors were used for the fixed effects with the expected value of 0 and variance $10^{12}$. Inverse-Wishart priors were used for the variances with the expected value of $1 \cdot 10^{-6}$ and degree of belief of $k+1$, where $k$ is the dimension of the covariance matrix. The number of iterations was 5,500,000, with a burnin of 500,000 and a thinning of 5000 .

\begin{tabular}{|c|c|c|c|c|c|c|c|}
\hline \multicolumn{8}{|c|}{ (a) $F_{1}$-adult dry mass } \\
\hline \multicolumn{8}{|c|}{ Fixed effects } \\
\hline & $\beta_{\text {post.mean }}$ & $2.5 \% \mathrm{Cl}$ & \multicolumn{2}{|c|}{$97.5 \% \mathrm{Cl}$} & eff.samp & $P_{\mathrm{MCMC}}$ & Sig. \\
\hline intercept & 0.100 & 0.092 & \multicolumn{2}{|c|}{0.108} & 1000.000 & 0.001 & $* * *$ \\
\hline LF treatment & 0.004 & -0.007 & \multicolumn{2}{|c|}{0.016} & 1000.000 & 0.496 & n.s. \\
\hline male sex & -0.074 & -0.086 & \multicolumn{2}{|c|}{-0.061} & 1000.000 & 0.001 & $* * *$ \\
\hline LF treatment $\times$ male sex & -0.004 & -0.019 & & 014 & 1000.000 & 0.626 & n.s. \\
\hline \multicolumn{8}{|c|}{ Residual (co)variances } \\
\hline & $\sigma_{\text {post.mean }}^{2}$ & ean $2.5 \%$ & \multicolumn{2}{|c|}{$\mathrm{Cl} \quad 97.5 \% \mathrm{Cl}$} & \multicolumn{2}{|c|}{ eff.samp } & \\
\hline$\theta_{\text {dry mass,dry mass }}$ & 0.0002 & 0.00 & & 0.0003 & \multicolumn{2}{|c|}{1000.0000} & \\
\hline \multirow{2}{*}{$\begin{array}{l}\theta_{\text {dry mass,lean mass }} \\
\theta_{\text {dry mass body fat }}\end{array}$} & 0.0001 & 0.00 & & 0.0001 & \multicolumn{2}{|c|}{1000.0000} & \\
\hline & 0.0003 & 0.00 & & 0.0004 & \multicolumn{2}{|c|}{1000.0000} & \\
\hline
\end{tabular}

\begin{tabular}{rcccccc}
\hline \multicolumn{10}{c}{ (b) $\mathbf{F}_{1}$-adult lean mass } \\
\hline \multicolumn{7}{c}{ Fixed effects } \\
intercept & 0.068 & 0.063 & 0.072 & 985.017 & 0.001 & $* * *$ \\
LF treatment & 0.000 & -0.006 & 0.008 & 900.715 & 0.964 & n.s. \\
male sex & -0.046 & -0.053 & -0.039 & 1000.000 & 0.001 & $* * *$ \\
LF treatment $\times$ male sex & -0.001 & -0.011 & 0.008 & 1000.000 & 0.780 & n.s. \\
\hline
\end{tabular}

\begin{tabular}{lcccc}
\hline \multicolumn{5}{c}{ Residual (co)variances } \\
\hline & $\sigma_{\text {post.mean }}^{2}$ & $2.5 \% \mathrm{Cl}$ & $97.5 \% \mathrm{Cl}$ & eff.samp \\
\hline$\theta_{\text {lean mass,lean mass }}$ & 0.0001 & 0.0000 & 0.0001 & 1000.0000 \\
$\theta_{\text {lean mass,dry mass }}$ & 0.0001 & 0.0000 & 0.0001 & 1000.0000 \\
$\theta_{\text {lean mass,body fat }}$ & 0.0001 & 0.0000 & 0.0002 & 1000.0000 \\
\hline
\end{tabular}




\begin{tabular}{rcccccc}
\hline \multicolumn{10}{c}{ (c) $\mathbf{F}_{1}$-adult body fat } \\
\hline Fixed effects \\
intercept & 0.177 & 0.163 & 0.194 & 852.729 & 0.001 & $* * *$ \\
LF treatment & 0.010 & -0.010 & 0.033 & 1169.723 & 0.388 & n.s. \\
male sex & -0.114 & -0.136 & -0.091 & 1000.000 & 0.001 & $* * *$ \\
LF treatment $\times$ male sex & -0.006 & -0.039 & 0.024 & 1000.000 & 0.716 & n.s. \\
\hline
\end{tabular}

\begin{tabular}{ccccc}
\hline \multicolumn{5}{c}{ Residual (co)variances } \\
\hline & $\sigma_{\text {post.mean }}^{2}$ & $2.5 \% \mathrm{Cl}$ & $97.5 \% \mathrm{Cl}$ & eff.samp \\
\hline$\theta_{\text {body fat,body fat }}$ & 0.0006 & 0.0004 & 0.0009 & 1000.0000 \\
$\theta_{\text {body fat,dry mass }}$ & 0.0003 & 0.0002 & 0.0004 & 1000.0000 \\
$\theta_{\text {body fat,lean mass }}$ & 0.0001 & 0.0000 & 0.0002 & 1000.0000 \\
\hline
\end{tabular}

$\beta_{\text {post.mean }}$ : posterior mean of regression coefficient; $\mathrm{Cl}$ : confidence interval; eff.samp: effective sample size; $P_{\mathrm{MCMC}}$ : posterior bayesian $p$-value; LF: low food; $\theta$ : residual (co)variance between two responses; significant codes: $P_{\mathrm{MCMC}} \leq 0.001^{* * *},<0.01^{* *}, \leq 0.05^{*},>0.05$ n.s. 
Table S.6.6. Fixed and random effects in explaining variation in the probability of offspring to respond to the stimulus during the fast-start trials estimated in a generalized linear mixed effects model using the MCMCglmm package (Hadfield, 2010). A logit link was used for the Bernoullidistributed response. The default normal priors were used for the fixed effects with the expected value of 0 and variance $10^{12}$. Inverse-Wishart priors were used for the variances with the expected value of 1 and degree of belief of 0.002 . As with binary models the residual variance is not identified, it was fixed at 1 . The number of iterations was $1,500,000$, with a burnin of 500,000 and a thinning of 1000 .

\begin{tabular}{|c|c|c|c|c|c|}
\hline \multicolumn{6}{|c|}{ Probability to respond during the fast-start trial } \\
\hline \multicolumn{6}{|c|}{ Fixed effects } \\
\hline & $\beta_{\text {post.mean }}$ & $2.5 \% \mathrm{Cl}$ & $97.5 \% \mathrm{Cl}$ & eff.samp & $P_{\mathrm{M}}$ \\
\hline intercept & -1.533 & -3.278 & -0.098 & 1000.000 & 0.0 \\
\hline LF treatment & -0.171 & -2.524 & 1.770 & 805.119 & \\
\hline experimental day & 0.044 & -0.004 & 0.089 & 1108.585 & 0.0 \\
\hline LF treatment $\times$ experimental day & -0.010 & -0.080 & 0.061 & 860.301 & \\
\hline \multicolumn{6}{|c|}{ Random effects } \\
\hline & $\sigma_{\text {post.mean }}^{2}$ & $2.5 \% \mathrm{Cl}$ & $97.5 \% \mathrm{Cl}$ & eff.samp & \\
\hline mother identity & 0.5714 & 0.0003 & 2.1054 & 1000.000 & \\
\hline Petri dish position & 0.2482 & 0.0003 & 0.8414 & 1000.000 & \\
\hline offspring identity & 1.8027 & 0.0003 & 5.3037 & 904.7566 & \\
\hline replicate trial identity & 0.3994 & 0.0003 & 0.9664 & $736.671 \mathrm{c}$ & \\
\hline
\end{tabular}

$\beta_{\text {post.mean: }}$ posterior mean of regression coefficient; $\mathrm{Cl}$ : confidence interval; eff.samp: effective sample size; $P_{\mathrm{MCMC}}$ : posterior bayesian $p$-value; LF: low food; significant codes: $P_{\text {MCMC }} \leq$ $0.001^{* * *},<0.01^{* *}, \leq 0.05^{*},>0.05$ n.s. 
Table S.6.7. Fixed and random effects in explaining variation in $\mathrm{F}_{1}$-offspring (a) In-transformed mean speed, (b) In-transformed maximum speed, (c) In-transformed mean acceleration, and (d) Intransformed maximum acceleration during fast-start escape response estimated using the MCMCglmm package (Hadfield, 2010). All traits were fitted in a multivariate linear mixed effects model allowing for the covariance between the residuals of all responses. The default normal priors were used for the fixed effects with the expected value of 0 and variance $10^{12}$. Inverse-Wishart priors were used for the variances with the expected value of $1 \cdot 10^{-6}$ and degree of belief of $k+1$, where $k$ is the dimension of the covariance matrix. The number of iterations was $5,500,000$, with a burnin of 500,000 and a thinning of 5000 .

\begin{tabular}{|c|c|c|c|c|c|c|}
\hline \multicolumn{7}{|c|}{ (a) $F_{1}$-mean speed } \\
\hline \multicolumn{7}{|c|}{ Fixed effects } \\
\hline & $\beta_{\text {post.mean }} 2$ & $2.5 \% \mathrm{Cl}$ & $97.5 \% \mathrm{C}$ & eff.samp & $P_{\mathrm{MCMC}}$ & Sig. \\
\hline intercept & -3.339 & -3.488 & -3.163 & 1000.000 & 0.001 & $* * *$ \\
\hline LF treatment & 0.187 & -0.124 & 0.488 & 1000.000 & 0.224 & n.s. \\
\hline experimental day & 0.003 & -0.003 & 0.009 & 1000.000 & 0.336 & n.s. \\
\hline LF treatment $\times$ experimental day & -0.003 & -0.014 & 0.007 & 1000.000 & 0.546 & n.s. \\
\hline \multicolumn{7}{|c|}{ Random effects } \\
\hline & $\sigma_{\text {post.mean }}^{2}$ & \multicolumn{2}{|c|}{$2.5 \% \mathrm{Cl}$} & $97.5 \% \mathrm{Cl}$ & \multicolumn{2}{|c|}{ eff.samp } \\
\hline mother identity & $0.0017 \times 10^{-3}$ & \multicolumn{2}{|c|}{$0.0003 \times 10^{-3}$} & $0.0044 \times 10^{-3}$ & \multicolumn{2}{|c|}{1000.0000} \\
\hline Petri dish position & $0.0016 \times 10^{-3}$ & \multicolumn{2}{|c|}{$0.0003 \times 10^{-3}$} & $0.0045 \times 10^{-3}$ & \multicolumn{2}{|c|}{1000.0000} \\
\hline offspring identity & $0.0017 \times 10^{-3}$ & \multicolumn{2}{|c|}{$0.0003 \times 10^{-3}$} & $0.0045 \times 10^{-3}$ & \multicolumn{2}{|c|}{1000.0000} \\
\hline replicate trial identity & $0.0016 \times 10^{-3}$ & \multicolumn{2}{|c|}{$0.0003 \times 10^{-3}$} & $0.0043 \times 10^{-3}$ & \multicolumn{2}{|c|}{734.9100} \\
\hline$\theta_{\text {mean speed,mean speed }}$ & 0.0842 & \multicolumn{2}{|c|}{0.0551} & 0.1210 & \multicolumn{2}{|c|}{906.6952} \\
\hline$\theta_{\text {mean speed,maximum speed }}$ & 0.0790 & \multicolumn{2}{|c|}{0.0500} & 0.1146 & \multicolumn{2}{|c|}{1000.0000} \\
\hline$\theta_{\text {mean speed,mean acceleration }}$ & 0.0931 & \multicolumn{2}{|c|}{0.0558} & 0.1405 & \multicolumn{2}{|c|}{1000.0000} \\
\hline$\theta_{\text {mean speed,maximum acceleration }}$ & 0.0883 & \multicolumn{2}{|c|}{0.0515} & 0.1343 & \multicolumn{2}{|c|}{1000.0000} \\
\hline
\end{tabular}


Supplementary Information

(b) $\mathbf{F}_{1}$-maximum speed

Fixed effects

\begin{tabular}{rcccccc}
\hline & $\beta_{\text {post.mean }}$ & $2.5 \% \mathrm{Cl}$ & $97.5 \% \mathrm{Cl}$ & eff.samp & $P_{\mathrm{MCMC}}$ & Sig. \\
intercept & -1.858 & -2.035 & -1.678 & 1000.000 & 0.001 & $* * *$ \\
LF treatment & 0.145 & -0.184 & 0.473 & 1000.000 & 0.348 & n.s. \\
experimental day & 0.003 & -0.003 & 0.010 & 1000.000 & 0.312 & n.s. \\
LF treatment $\times$ experimental day & -0.005 & -0.016 & 0.006 & 1000.000 & 0.368 & n.s. \\
\hline
\end{tabular}

\begin{tabular}{lcccc}
\hline \multicolumn{5}{c}{ Random effects } \\
\hline & $\sigma_{\text {post.mean }}^{2}$ & $2.5 \% \mathrm{Cl}$ & $97.5 \% \mathrm{Cl}$ & eff.samp \\
\hline mother identity & $0.0017 \times 10^{-3}$ & $0.0002 \times 10^{-3}$ & $0.0049 \times 10^{-3}$ & 915.8391 \\
Petri dish position & $0.0018 \times 10^{-3}$ & $0.0003 \times 10^{-3}$ & $0.0048 \times 10^{-3}$ & 1000.0000 \\
offspring identity & $0.0016 \times 10^{-3}$ & $0.0002 \times 10^{-3}$ & $0.0043 \times 10^{-3}$ & 1000.0000 \\
replicate trial identity & $0.0017 \times 10^{-3}$ & $0.0003 \times 10^{-3}$ & $0.0045 \times 10^{-3}$ & 1000.0000 \\
$\theta_{\text {maximum speed,maximum speed }}$ & 0.0942 & 0.0599 & 0.1330 & 1000.0000 \\
$\theta_{\text {maximum speed,mean speed }}$ & 0.0790 & 0.0500 & 0.1146 & 1000.0000 \\
$\theta_{\text {maximum speed,mean acceleration }}$ & 0.1146 & 0.0689 & 0.1661 & 1000.0000 \\
$\theta_{\text {maximum speed,maximum acceleration }}$ & 0.1177 & 0.0748 & 0.1693 & 1000.0000 \\
\hline
\end{tabular}

\section{(c) $F_{1}$-mean acceleration}

Fixed effects

\begin{tabular}{rcccccc}
\hline & $\beta_{\text {post.mean }}$ & $2.5 \% \mathrm{Cl}$ & $97.5 \% \mathrm{Cl}$ & eff.samp & $P_{\mathrm{MCMC}}$ & Sig. \\
intercept & 0.733 & 0.475 & 0.975 & 1000.000 & 0.001 & $* * *$ \\
LF treatment & -0.026 & -0.475 & 0.436 & 1000.000 & 0.904 & n.s. \\
experimental day & -0.003 & -0.012 & 0.005 & 1000.000 & 0.464 & n.s. \\
LF treatment $\times$ experimental day & 0.002 & -0.014 & 0.016 & 1000.000 & 0.838 & n.s. \\
\hline
\end{tabular}

\begin{tabular}{lcccc}
\hline \multicolumn{5}{c}{ Random effects } \\
\hline & $\sigma_{\text {post.mean }}^{2}$ & $2.5 \% \mathrm{Cl}$ & $97.5 \% \mathrm{Cl}$ & eff.samp \\
\hline mother identity & $0.0017 \times 10^{-3}$ & $0.0002 \times 10^{-3}$ & $0.0047 \times 10^{-3}$ & 633.6177 \\
Petri dish position & $0.0017 \times 10^{-3}$ & $0.0003 \times 10^{-3}$ & $0.0046 \times 10^{-3}$ & 1000.0000 \\
offspring identity & $0.0017 \times 10^{-3}$ & $0.0002 \times 10^{-3}$ & $0.0042 \times 10^{-3}$ & 914.0502 \\
replicate trial identity & $0.0017 \times 10^{-3}$ & $0.0003 \times 10^{-3}$ & $0.0044 \times 10^{-3}$ & 1000.0000 \\
$\theta_{\text {mean acceleration,mean acceleration }}$ & 0.1839 & 0.1173 & 0.2605 & 1000.0000 \\
$\theta_{\text {mean acceleration,mean speed }}$ & 0.0931 & 0.0558 & 0.1405 & 1000.0000 \\
$\theta_{\text {mean acceleration,maximum speed }}$ & 0.1146 & 0.0689 & 0.1661 & 1000.0000 \\
$\theta_{\text {mean acceleration,maximum acceleration }}$ & 0.1686 & 0.1047 & 0.2384 & 1000.0000 \\
\hline
\end{tabular}




\begin{tabular}{|c|c|c|c|c|c|c|}
\hline \multicolumn{7}{|c|}{ (d) $F_{1}$-maximum acceleration } \\
\hline \multicolumn{7}{|c|}{ Fixed effects } \\
\hline & $\overline{\beta_{\text {post.mean }}}$ & $2.5 \% \mathrm{Cl}$ & $97.5 \% \mathrm{Cl}$ & eff.samp & $P_{\mathrm{MCMC}}$ & Sig. \\
\hline intercept & 2.124 & 1.910 & 2.391 & 975.217 & 0.001 & $* * *$ \\
\hline LF treatment & 0.163 & -0.281 & 0.616 & 1000.000 & 0.482 & n.s. \\
\hline experimental day & -0.003 & -0.012 & 0.005 & 860.262 & 0.492 & n.s. \\
\hline LF treatment $\times$ experimental day & -0.004 & -0.019 & 0.011 & 1000.000 & 0.656 & n.s. \\
\hline \multicolumn{7}{|c|}{ Random effects } \\
\hline & \multicolumn{2}{|c|}{$\sigma_{\text {post.mean }}^{2}$} & $2.5 \% \mathrm{Cl}$ & \multicolumn{2}{|c|}{$97.5 \% \mathrm{Cl}$} & eff.samp \\
\hline mother identity & \multicolumn{2}{|c|}{$0.0016 \times 10^{-3}$} & $0.0002 \times 10^{-3}$ & \multicolumn{2}{|c|}{$0.0044 \times 10^{-3}$} & 831.3166 \\
\hline Petri dish position & \multicolumn{2}{|c|}{$0.0017 \times 10^{-3}$} & $0.0003 \times 10^{-3}$ & \multicolumn{2}{|c|}{$0.0045 \times 10^{-3}$} & 1000.0000 \\
\hline offspring identity & \multicolumn{2}{|c|}{$0.0016 \times 10^{-3}$} & $0.0003 \times 10^{-3}$ & \multicolumn{2}{|c|}{$0.0041 \times 10^{-3}$} & 1000.0000 \\
\hline replicate trial identity & \multicolumn{2}{|c|}{$0.0017 \times 10^{-3}$} & $0.0003 \times 10^{-3}$ & \multicolumn{2}{|c|}{$0.0047 \times 10^{-3}$} & 1000.0000 \\
\hline$\theta_{\text {maximum acceleration,maximum acceleration }}$ & \multicolumn{2}{|c|}{0.1745} & 0.1089 & \multicolumn{2}{|c|}{0.2402} & 1000.0000 \\
\hline$\theta_{\text {maximum acceleration,mean speed }}$ & \multicolumn{2}{|c|}{0.0883} & 0.0515 & \multicolumn{2}{|c|}{0.1343} & 1000.0000 \\
\hline$\theta_{\text {maximum acceleration,maximum speed }}$ & \multicolumn{2}{|c|}{0.1177} & 0.0748 & \multicolumn{2}{|c|}{0.1693} & 1000.0000 \\
\hline$\theta_{\text {maximum acceleration,mean acceleration }}$ & \multicolumn{2}{|c|}{0.1686} & 0.1047 & \multicolumn{2}{|c|}{0.2384} & 1000.0000 \\
\hline
\end{tabular}

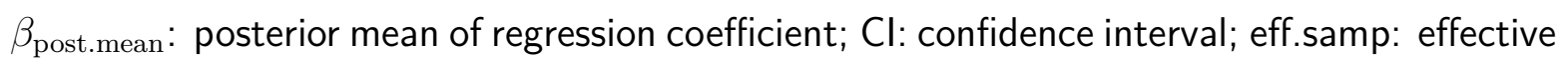
sample size; $P_{\mathrm{MCMC}}$ : posterior bayesian $p$-value; LF: low food; $\theta$ : residual (co)variance between two responses; significant codes: $P_{\mathrm{MCMC}} \leq 0.001^{* * *},<0.01^{* *}, \leq 0.05^{*},>0.05$ n.s. 
Table S.6.8. Fixed and random effects in explaining variation in $F_{1}$-offspring (a) In-transformed mean speed, (b) In-transformed maximum speed, (c) In-transformed mean acceleration, and (d) In-transformed maximum acceleration during food supply estimated using the MCMCglmm package (Hadfield, 2010). All traits were fitted in a multivariate linear mixed effects model allowing for the covariance between the residuals of all responses. The default normal priors were used for the fixed effects with the expected value of 0 and variance $10^{12}$. Inverse-Wishart priors were used for the variances with the expected value of $1 \cdot 10^{-6}$ and degree of belief of $k+1$, where $k$ is the dimension of the covariance matrix. The number of iterations was 5,500,000, with a burnin of 500,000 and a thinning of 5000 .

\begin{tabular}{|c|c|c|c|c|c|c|}
\hline \multicolumn{7}{|c|}{ (a) $F_{1}$-mean speed } \\
\hline \multicolumn{7}{|c|}{ Fixed effects } \\
\hline & $\beta_{\text {post.mean }}$ & $2.5 \% \mathrm{Cl}$ & $97.5 \% \mathrm{Cl}$ & eff.samp & $P_{\mathrm{MCMC}}$ & Sig. \\
\hline intercept & -7.271 & -7.508 & -7.056 & 1096.112 & 0.001 & $* * *$ \\
\hline LF treatment & 0.604 & 0.304 & 0.906 & 1099.085 & 0.001 & $* * *$ \\
\hline experimental day & 0.012 & 0.005 & 0.019 & 939.113 & 0.001 & $* * *$ \\
\hline day 2 after birth & 1.044 & 0.819 & 1.261 & 1000.000 & 0.001 & $* * *$ \\
\hline day 7 after birth & 1.374 & 1.180 & 1.615 & 1000.000 & 0.001 & $* * *$ \\
\hline LF treatment $\times$ experimental day & -0.012 & -0.021 & -0.003 & 1153.552 & 0.014 & $*$ \\
\hline LF treatment $\times$ day 2 after birth & -0.339 & -0.624 & -0.043 & 1000.000 & 0.018 & $*$ \\
\hline LF treatment $\times$ day 7 after birth & -0.377 & -0.650 & -0.067 & 1000.000 & 0.012 & $*$ \\
\hline \multicolumn{7}{|c|}{ Random effects } \\
\hline & $\sigma_{\text {post.mean }}^{2}$ & \multicolumn{2}{|c|}{$2.5 \% \mathrm{Cl}$} & $97.5 \% \mathrm{Cl}$ & \multicolumn{2}{|c|}{ eff.samp } \\
\hline mother identity & $0.0016 \times 10^{-3}$ & \multicolumn{2}{|c|}{$0.0003 \times 10^{-3}$} & $0.0043 \times 10^{-3}$ & \multicolumn{2}{|c|}{1000.0000} \\
\hline Petri dish position & $0.0018 \times 10^{-3}$ & \multicolumn{2}{|c|}{$0.0002 \times 10^{-3}$} & $0.0048 \times 10^{-3}$ & \multicolumn{2}{|c|}{1000.0000} \\
\hline offspring identity & $0.0017 \times 10^{-3}$ & \multicolumn{2}{|c|}{$0.0002 \times 10^{-3}$} & $0.0045 \times 10^{-3}$ & \multicolumn{2}{|c|}{1000.0000} \\
\hline$\theta_{\text {mean speed,mean speed }}$ & 0.3007 & \multicolumn{2}{|c|}{0.2540} & 0.3465 & \multicolumn{2}{|c|}{1000.0000} \\
\hline$\theta_{\text {mean speed,maximum speed }}$ & 0.1600 & \multicolumn{2}{|c|}{0.1322} & 0.1862 & \multicolumn{2}{|c|}{1000.0000} \\
\hline$\theta_{\text {mean speed,mean acceleration }}$ & 0.2337 & \multicolumn{2}{|c|}{0.1973} & 0.2703 & \multicolumn{2}{|c|}{1000.0000} \\
\hline$\theta_{\text {mean speed,maximum acceleration }}$ & 0.1362 & \multicolumn{2}{|c|}{0.1115} & 0.1627 & \multicolumn{2}{|c|}{1000.0000} \\
\hline
\end{tabular}




\begin{tabular}{|c|c|c|c|c|c|c|c|}
\hline \multicolumn{8}{|c|}{ (b) $F_{1}$-maximum speed } \\
\hline \multicolumn{8}{|c|}{ Fixed effects } \\
\hline & $\beta_{\text {post.mean }}$ & $2.5^{\circ}$ & ${ }_{0} \mathrm{Cl}$ & $97.5 \% \mathrm{Cl}$ & eff.samp & $P_{\mathrm{MCMC}}$ & Sig. \\
\hline intercept & -5.682 & -5 & 820 & -5.540 & 1000.000 & 0.001 & $* * *$ \\
\hline LF treatment & 0.318 & 0.1 & & 0.508 & 1000.000 & 0.004 & $* *$ \\
\hline experimental day & 0.007 & 0.0 & & 0.011 & 1182.131 & 0.002 & $* *$ \\
\hline day 2 after birth & 0.518 & 0.3 & & 0.650 & 1000.000 & 0.001 & $* * *$ \\
\hline day 7 after birth & 0.759 & 0.6 & & 0.896 & 1000.000 & 0.001 & $* * *$ \\
\hline LF treatment $\times$ experimental day & -0.008 & -0 & 014 & -0.002 & 1385.756 & 0.012 & $*$ \\
\hline LF treatment $\times$ day 2 after birth & -0.174 & -0 & 353 & 0.021 & 1000.000 & 0.070 & n.s. \\
\hline LF treatment $\times$ day 7 after birth & -0.184 & -0 & 362 & 0.002 & 1000.000 & 0.056 & n.s. \\
\hline \multicolumn{8}{|c|}{ Random effects } \\
\hline & \multicolumn{2}{|c|}{$\sigma_{\text {post.mean }}^{2}$} & \multicolumn{2}{|c|}{$2.5 \% \mathrm{Cl}$} & $97.5 \% \mathrm{Cl}$ & \multicolumn{2}{|c|}{ eff.samp } \\
\hline mother identity & \multicolumn{2}{|c|}{$0.0017 \times 10^{-3}$} & \multicolumn{2}{|c|}{$0.0003 \times 10^{-3}$} & $0.0050 \times 10^{-3}$ & \multicolumn{2}{|c|}{1000.0000} \\
\hline Petri dish position & \multicolumn{2}{|c|}{$0.0017 \times 10^{-3}$} & \multicolumn{2}{|c|}{$0.0003 \times 10^{-3}$} & $0.0046 \times 10^{-3}$ & \multicolumn{2}{|c|}{1000.0000} \\
\hline offspring identity & \multicolumn{2}{|c|}{$0.0016 \times 10^{-3}$} & \multicolumn{2}{|c|}{$0.0003 \times 10^{-3}$} & $0.0042 \times 10^{-3}$ & \multicolumn{2}{|c|}{721.8976} \\
\hline$\theta_{\text {maximum speed,maximum speed }}$ & \multicolumn{2}{|c|}{0.1183} & \multicolumn{2}{|c|}{0.1008} & 0.1365 & \multicolumn{2}{|c|}{1000.0000} \\
\hline$\theta_{\text {maximum speed,mean speed }}$ & \multicolumn{2}{|c|}{0.1600} & \multicolumn{2}{|r|}{0.1322} & 0.1862 & \multicolumn{2}{|c|}{1000.0000} \\
\hline$\theta_{\text {maximum speed,mean acceleration }}$ & \multicolumn{2}{|c|}{0.1409} & \multicolumn{2}{|r|}{0.1186} & 0.1643 & \multicolumn{2}{|c|}{1000.0000} \\
\hline$\theta_{\text {maximum speed,maximum acceleration }}$ & \multicolumn{2}{|c|}{0.1100} & \multicolumn{2}{|r|}{0.0933} & 0.1280 & \multicolumn{2}{|c|}{1000.0000} \\
\hline
\end{tabular}

\section{(c) $F_{1}$-mean acceleration}

\begin{tabular}{|c|c|c|c|c|c|c|}
\hline \multicolumn{7}{|c|}{ Fixed effects } \\
\hline & $\beta_{\text {post.mean }}$ & $2.5 \% \mathrm{Cl}$ & $97.5 \% \mathrm{Cl}$ & eff.samp & $P_{\mathrm{MCMC}}$ & Sig. \\
\hline intercept & -7.980 & -8.165 & -7.795 & 1226.138 & 0.001 & $* * *$ \\
\hline LF treatment & 0.423 & 0.158 & 0.675 & 1180.552 & 0.006 & $* *$ \\
\hline experimental day & 0.008 & 0.002 & 0.014 & 990.127 & 0.010 & $*$ \\
\hline day 2 after birth & 0.800 & 0.617 & 0.994 & 1201.528 & 0.001 & *** \\
\hline day 7 after birth & 1.059 & 0.893 & 1.250 & 1251.870 & 0.001 & $* * *$ \\
\hline LF treatment $\times$ experimental day & -0.009 & -0.017 & -0.002 & 1265.511 & 0.018 & $*$ \\
\hline LF treatment $\times$ day 2 after birth & -0.246 & -0.529 & -0.037 & 1000.000 & 0.050 & $*$ \\
\hline LF treatment $\times$ day 7 after birth & -0.273 & \multicolumn{2}{|c|}{-0.511} & 1000.000 & 0.034 & $*$ \\
\hline \multicolumn{7}{|c|}{ Random effects } \\
\hline & \multicolumn{2}{|c|}{$\sigma_{\text {post.mean }}^{2}$} & $2.5 \% \mathrm{Cl}$ & $97.5 \% \mathrm{Cl}$ & \multicolumn{2}{|c|}{ eff.samp } \\
\hline mother identity & \multicolumn{2}{|c|}{$0.0017 \times 10^{-3}$} & $0.0002 \times 10^{-3}$ & $0.0044 \times 10^{-3}$ & \multicolumn{2}{|c|}{948.6556} \\
\hline Petri dish position & \multicolumn{2}{|c|}{$0.0017 \times 10^{-3}$} & $0.0003 \times 10^{-3}$ & $0.0044 \times 10^{-3}$ & \multicolumn{2}{|c|}{1000.0000} \\
\hline offspring identity & \multicolumn{2}{|c|}{$0.0017 \times 10^{-3}$} & $0.0003 \times 10^{-3}$ & $0.0049 \times 10^{-3}$ & \multicolumn{2}{|c|}{1000.0000} \\
\hline$\theta_{\text {mean acceleration,mean acceleration }}$ & \multicolumn{2}{|c|}{0.2045} & 0.1733 & 0.2362 & \multicolumn{2}{|c|}{1000.0000} \\
\hline$\theta_{\text {mean acceleration,mean speed }}$ & \multicolumn{2}{|c|}{0.2337} & 0.1973 & 0.2703 & \multicolumn{2}{|c|}{1000.0000} \\
\hline$\theta_{\text {mean acceleration,maximum speed }}$ & \multicolumn{2}{|c|}{0.1409} & 0.1186 & 0.1643 & \multicolumn{2}{|c|}{1000.0000} \\
\hline$\theta_{\text {mean acceleration,maximum acceleration }}$ & \multicolumn{2}{|c|}{0.1328} & 0.1075 & 0.1533 & \multicolumn{2}{|c|}{1000.0000} \\
\hline
\end{tabular}


(d) $\mathbf{F}_{1}$-maximum acceleration

\begin{tabular}{rcccccc}
\hline \multicolumn{7}{c}{ Fixed effects } \\
intercept & $\beta_{\text {post.mean }}$ & $2.5 \% \mathrm{Cl}$ & $97.5 \% \mathrm{Cl}$ & eff.samp & $P_{\mathrm{MCMC}}$ & Sig. \\
LF treatment & 0.247 & -6.482 & -6.205 & 1000.000 & 0.001 & $* * *$ \\
experimental day & 0.005 & 0.036 & 0.432 & 1092.713 & 0.022 & $*$ \\
day 2 after birth & 0.430 & 0.300 & 0.584 & 1000.000 & 0.001 & $* * *$ \\
day 7 after birth & 0.603 & 0.469 & 0.738 & 1000.000 & 0.001 & $* * *$ \\
LF treatment $\times$ experimental day & -0.006 & -0.011 & -0.000 & 1332.997 & 0.052 & n.s. \\
LF treatment $\times$ day 2 after birth & -0.166 & -0.339 & 0.029 & 1000.000 & 0.078 & n.s. \\
LF treatment $\times$ day 7 after birth & -0.147 & -0.326 & 0.035 & 1000.000 & 0.100 & n.s. \\
\hline
\end{tabular}

\begin{tabular}{lcccc}
\hline \multicolumn{5}{c}{ Random effects } \\
\hline & $\sigma_{\text {post.mean }}^{2}$ & $2.5 \% \mathrm{Cl}$ & $97.5 \% \mathrm{Cl}$ & eff.samp \\
\hline mother identity & $0.0017 \times 10^{-3}$ & $0.0003 \times 10^{-3}$ & $0.0049 \times 10^{-3}$ & 721.9755 \\
Petri dish position & $0.0016 \times 10^{-3}$ & $0.0002 \times 10^{-3}$ & $0.0044 \times 10^{-3}$ & 1093.6333 \\
offspring identity & $0.0017 \times 10^{-3}$ & $0.0003 \times 10^{-3}$ & $0.0045 \times 10^{-3}$ & 855.2492 \\
$\theta_{\text {maximum acceleration,maximum acceleration }}$ & 0.1158 & 0.0966 & 0.1330 & 1000.0000 \\
$\theta_{\text {maximum acceleration,mean speed }}$ & 0.1362 & 0.1115 & 0.1627 & 1000.0000 \\
$\theta_{\text {maximum acceleration,maximum speed }}$ & 0.1100 & 0.0933 & 0.1280 & 1000.0000 \\
$\theta_{\text {maximum acceleration,mean acceleration }}$ & 0.1328 & 0.1075 & 0.1533 & 1000.0000 \\
\hline
\end{tabular}

$\beta_{\text {post.mean }}$ : posterior mean of regression coefficient; $\mathrm{Cl}$ : confidence interval; eff.samp: effective sample size; $P_{\mathrm{MCMC}}$ : posterior bayesian $p$-value; LF: low food; $\theta$ : residual (co)variance between two responses; significant codes: $P_{\mathrm{MCMC}} \leq 0.001^{* * *},<0.01^{* *}, \leq 0.05^{*},>0.05$ n.s. 
Table S.6.9. Fixed and random effects in explaining variation in $F_{1}$-offspring (a) In-transformed mean speed, (b) In-transformed maximum speed, (c) In-transformed mean acceleration, and (d) In-transformed maximum acceleration during the 5 min control period (no food supply) estimated using the MCMCglmm package (Hadfield, 2010). All traits were fitted in a multivariate linear mixed effects model allowing for the covariance between the residuals of all responses. The default normal priors were used for the fixed effects with the expected value of 0 and variance $10^{12}$. Inverse-Wishart priors were used for the variances with the expected value of $1 \cdot 10^{-6}$ and degree of belief of $k+1$, where $k$ is the dimension of the covariance matrix. The number of iterations was 5,500,000, with a burnin of 500,000 and a thinning of 5000 .

\begin{tabular}{|c|c|c|c|c|c|c|}
\hline \multicolumn{7}{|c|}{ (a) $F_{1-\text { mean speed }}$} \\
\hline \multicolumn{7}{|c|}{ Fixed effects } \\
\hline & $\beta_{\text {post.mean }} 2$ & $2.5 \% \mathrm{Cl}$ & $97.5 \% \mathrm{Cl}$ & eff.samp & $P_{\mathrm{MCMC}}$ & Sig. \\
\hline intercept & -7.130 & -7.457 & -6.841 & 1044.230 & 0.001 & $* * *$ \\
\hline LF treatment & 0.227 & -0.193 & 0.629 & 1000.000 & 0.244 & n.s. \\
\hline experimental day & 0.004 & -0.005 & 0.013 & 1000.000 & 0.356 & n.s. \\
\hline day 2 after birth & 1.144 & 0.846 & 1.456 & 838.250 & 0.001 & $* * *$ \\
\hline day 7 after birth & 1.542 & 1.255 & 1.880 & 1100.634 & 0.001 & $* * *$ \\
\hline LF treatment $\times$ experimental day & 0.000 & -0.012 & 0.012 & 1000.000 & 0.966 & n.s. \\
\hline LF treatment $\times$ day 2 after birth & -0.234 & -0.659 & 0.159 & 1000.000 & 0.252 & n.s. \\
\hline LF treatment $\times$ day 7 after birth & -0.297 & -0.695 & 0.106 & 1167.894 & 0.156 & n.s. \\
\hline \multicolumn{7}{|c|}{ Random effects } \\
\hline & $\sigma_{\text {post.mean }}^{2}$ & \multicolumn{2}{|c|}{$2.5 \% \mathrm{Cl}$} & $97.5 \% \mathrm{Cl}$ & \multicolumn{2}{|c|}{ eff.samp } \\
\hline mother identity & $0.0016 \times 10^{-3}$ & \multicolumn{2}{|c|}{$0.0003 \times 10^{-3}$} & $0.0047 \times 10^{-3}$ & \multicolumn{2}{|c|}{1000.0000} \\
\hline Petri dish position & $0.0017 \times 10^{-3}$ & \multicolumn{2}{|c|}{$0.0002 \times 10^{-3}$} & $0.0045 \times 10^{-3}$ & \multicolumn{2}{|c|}{1000.0000} \\
\hline offspring identity & $0.0016 \times 10^{-3}$ & \multicolumn{2}{|c|}{$0.0002 \times 10^{-3}$} & $0.0041 \times 10^{-3}$ & \multicolumn{2}{|c|}{881.8610} \\
\hline$\theta_{\text {mean speed,mean speed }}$ & 0.5953 & \multicolumn{2}{|c|}{0.5072} & 0.6991 & \multicolumn{2}{|c|}{1862.0378} \\
\hline$\theta_{\text {mean speed,maximum speed }}$ & 0.3393 & \multicolumn{2}{|c|}{0.2886} & 0.4029 & \multicolumn{2}{|c|}{1538.1364} \\
\hline$\theta_{\text {mean speed,mean acceleration }}$ & 0.5003 & \multicolumn{2}{|c|}{0.4239} & 0.5877 & \multicolumn{2}{|c|}{1000.0000} \\
\hline$\theta_{\text {mean speed,maximum acceleration }}$ & 0.3068 & \multicolumn{2}{|c|}{0.2608} & 0.3682 & \multicolumn{2}{|c|}{1000.0000} \\
\hline
\end{tabular}


Supplementary Information

\begin{tabular}{|c|c|c|c|c|c|c|c|}
\hline \multicolumn{8}{|c|}{ (b) $F_{1}$-maximum speed } \\
\hline \multicolumn{8}{|c|}{ Fixed effects } \\
\hline & $\beta_{\text {post.mean }}$ & $2.5 \%$ & $\mathrm{Cl}$ & $97.5 \% \mathrm{Cl}$ & eff.samp & $\overline{P_{\mathrm{MCMC}}}$ & Sig. \\
\hline intercept & -5.646 & -5.8 & & -5.460 & 1056.531 & 0.001 & $* * *$ \\
\hline LF treatment & 0.081 & -0.1 & 154 & 0.354 & 1000.000 & 0.522 & n.s. \\
\hline experimental day & 0.003 & -0.0 & 003 & 0.008 & 735.624 & 0.364 & n.s. \\
\hline day 2 after birth & 0.600 & 0.40 & & 0.792 & 872.418 & 0.001 & $* * *$ \\
\hline day 7 after birth & 0.868 & 0.66 & & 1.062 & 1000.000 & 0.001 & $* * *$ \\
\hline LF treatment $\times$ experimental day & -0.001 & -0.0 & 008 & 0.007 & 1000.000 & 0.912 & n.s. \\
\hline LF treatment $\times$ day 2 after birth & -0.128 & -0.3 & & 0.113 & 1000.000 & 0.350 & n.s. \\
\hline LF treatment $\times$ day 7 after birth & -0.114 & -0.3 & 398 & 0.125 & 1121.951 & 0.394 & n.s. \\
\hline \multicolumn{8}{|c|}{ Random effects } \\
\hline & \multicolumn{2}{|c|}{$\sigma_{\text {post.mean }}^{2}$} & \multicolumn{2}{|c|}{$2.5 \% \mathrm{Cl}$} & $97.5 \% \mathrm{Cl}$ & \multicolumn{2}{|c|}{ eff.samp } \\
\hline mother identity & \multicolumn{2}{|c|}{$0.0017 \times 10^{-3}$} & \multicolumn{2}{|c|}{$0.0003 \times 10^{-3}$} & $0.0047 \times 10^{-3}$ & \multicolumn{2}{|c|}{1000.0000} \\
\hline Petri dish position & \multicolumn{2}{|c|}{$0.0017 \times 10^{-3}$} & \multicolumn{2}{|c|}{$0.0003 \times 10^{-3}$} & $0.0045 \times 10^{-3}$ & \multicolumn{2}{|c|}{1000.0000} \\
\hline offspring identity & \multicolumn{2}{|c|}{$0.0016 \times 10^{-3}$} & \multicolumn{2}{|c|}{$0.0003 \times 10^{-3}$} & $0.0044 \times 10^{-3}$ & \multicolumn{2}{|c|}{1000.0000} \\
\hline$\theta_{\text {maximum speed,maximum speed }}$ & \multicolumn{2}{|c|}{0.2344} & \multicolumn{2}{|c|}{0.1990} & 0.2728 & \multicolumn{2}{|c|}{1000.0000} \\
\hline$\theta_{\text {maximum speed,mean speed }}$ & \multicolumn{2}{|c|}{0.3393} & \multicolumn{2}{|c|}{0.2886} & 0.4029 & \multicolumn{2}{|c|}{1538.1364} \\
\hline$\theta_{\text {maximum speed,mean acceleration }}$ & \multicolumn{2}{|c|}{0.3006} & & .2566 & 0.3577 & 1000 & 0000 \\
\hline$\theta_{\text {maximum speed,maximum acceleration }}$ & 0.218 & & & .1870 & 0.2576 & 1000. & 0000 \\
\hline & (c) $\mathbf{F}_{1}-\mathbf{m}$ & in acc & elera & & & & \\
\hline & Fixe & d effec & & & & & \\
\hline & $\beta_{\text {post.mean }}$ & $2.5 \%$ & $\mathrm{Cl}$ & $97.5 \% \mathrm{Cl}$ & eff.samp & $P_{\mathrm{MCMC}}$ & Sig. \\
\hline intercept & -7.998 & -8.2 & 267 & -7.733 & 1082.359 & 0.001 & $* * *$ \\
\hline LF treatment & 0.134 & -0.2 & & 0.491 & 1000.000 & 0.442 & n.s. \\
\hline experimental day & 0.004 & -0.0 & 004 & 0.011 & 1000.000 & 0.344 & n.s. \\
\hline day 2 after birth & 0.933 & 0.67 & & 1.213 & 906.856 & 0.001 & $* * *$ \\
\hline day 7 after birth & 1.294 & 1.01 & & 1.568 & 1099.627 & 0.001 & $* * *$ \\
\hline LF treatment $\times$ experimental day & -0.000 & -0.0 & 10 & 0.010 & 1000.000 & 1.000 & n.s. \\
\hline LF treatment $\times$ day 2 after birth & -0.157 & -0.4 & & 0.185 & 1000.000 & 0.386 & n.s. \\
\hline LF treatment $\times$ day 7 after birth & -0.199 & -0.5 & 540 & 0.152 & 1070.329 & 0.266 & n.s. \\
\hline & Randc & m effe & ects & & & & \\
\hline & $\sigma_{\text {post.m }}^{2}$ & & & $.5 \% \mathrm{Cl}$ & $97.5 \% \mathrm{Cl}$ & eff.s & $\mathrm{amp}$ \\
\hline mother identity & $0.0017 \times$ & $10^{-3}$ & 0.00 & $02 \times 10^{-3}$ & $0.0044 \times 10^{-3}$ & 1000 & 0000 \\
\hline Petri dish position & $0.0017 \times$ & $10^{-3}$ & 0.00 & $003 \times 10^{-3}$ & $0.0042 \times 10^{-3}$ & 1000 & .0000 \\
\hline offspring identity & $0.0016 \times$ & $10^{-3}$ & 0.00 & $03 \times 10^{-3}$ & $0.0044 \times 10^{-3}$ & 887 & 9253 \\
\hline$\theta_{\text {mean acceleration,mean acceleration }}$ & 0.445 & & & 0.3834 & 0.5279 & 1000 & 0000 \\
\hline$\theta_{\text {mean acceleration,mean speed }}$ & 0.500 & & & 0.4239 & 0.5877 & 1000 & 0000 \\
\hline$\theta_{\text {mean acceleration,maximum speed }}$ & 0.300 & & & 0.2566 & 0.3577 & 1000 & 0000 \\
\hline$\theta_{\text {mean acceleration,maximum acceleration }}$ & 0.284 & & & 0.2421 & 0.3380 & 1000 & 0000 \\
\hline
\end{tabular}




\begin{tabular}{|c|c|c|c|c|c|c|}
\hline \multicolumn{7}{|c|}{ (d) $F_{1}$-maximum acceleration } \\
\hline \multicolumn{7}{|c|}{ Fixed effects } \\
\hline & $\beta_{\text {post.mean }}$ & $2.5 \% \mathrm{Cl}$ & $97.5 \% \mathrm{Cl}$ & eff.samp & $P_{\mathrm{MCMC}}$ & Sig. \\
\hline intercept & -6.415 & -6.602 & -6.236 & 1109.278 & 0.001 & $* * *$ \\
\hline LF treatment & 0.039 & -0.197 & 0.280 & 1000.000 & 0.754 & n.s. \\
\hline experimental day & 0.002 & -0.003 & 0.008 & 953.635 & 0.392 & n.s. \\
\hline day 2 after birth & 0.571 & 0.370 & 0.741 & 940.379 & 0.001 & $* * *$ \\
\hline day 7 after birth & 0.822 & 0.630 & 1.015 & 1000.000 & 0.001 & $* * *$ \\
\hline LF treatment $\times$ experimental day & -0.000 & -0.007 & 0.007 & 1000.000 & 0.990 & n.s. \\
\hline LF treatment $\times$ day 2 after birth & -0.104 & -0.359 & 0.112 & 1000.000 & 0.398 & n.s. \\
\hline LF treatment $\times$ day 7 after birth & -0.074 & -0.333 & 0.172 & 1096.224 & 0.554 & n.s. \\
\hline \multicolumn{7}{|c|}{ Random effects } \\
\hline & \multicolumn{2}{|c|}{$\sigma_{\text {post.mean }}^{2}$} & $2.5 \% \mathrm{Cl}$ & \multicolumn{2}{|c|}{$97.5 \% \mathrm{Cl}$} & eff.samp \\
\hline mother identity & \multicolumn{2}{|c|}{$0.0017 \times 10^{-3}$} & $0.0003 \times 10^{-3}$ & \multicolumn{2}{|c|}{$0.0042 \times 10^{-3}$} & 1000.0000 \\
\hline Petri dish position & \multicolumn{2}{|c|}{$0.0017 \times 10^{-3}$} & $0.0002 \times 10^{-3}$ & \multicolumn{2}{|c|}{$0.0047 \times 10^{-3}$} & 1000.0000 \\
\hline offspring identity & \multicolumn{2}{|c|}{$0.0017 \times 10^{-3}$} & $0.0003 \times 10^{-3}$ & \multicolumn{2}{|c|}{$0.0042 \times 10^{-3}$} & 1000.0000 \\
\hline$\theta_{\text {maximum acceleration,maximum acceler }}$ & \multicolumn{2}{|c|}{0.2164} & 0.1831 & \multicolumn{2}{|c|}{0.2523} & 1000.0000 \\
\hline$\theta_{\text {maximum acceleration,mean speed }}$ & \multicolumn{2}{|c|}{0.3068} & 0.2608 & \multicolumn{2}{|c|}{0.3682} & 1000.0000 \\
\hline$\theta_{\text {maximum acceleration,maximum speed }}$ & \multicolumn{2}{|c|}{0.2184} & 0.1870 & \multicolumn{2}{|c|}{0.2576} & 1000.0000 \\
\hline$\theta_{\text {maximum acceleration,mean acceleration }}$ & \multicolumn{2}{|c|}{0.2846} & 0.2421 & \multicolumn{2}{|c|}{0.3380} & 1000.0000 \\
\hline
\end{tabular}

$\beta_{\text {post.mean }}$ : posterior mean of regression coefficient; $\mathrm{Cl}$ : confidence interval; eff.samp: effective sample size; $P_{\mathrm{MCMC}}$ : posterior bayesian $p$-value; LF: low food; $\theta$ : residual (co)variance between two responses; significant codes: $P_{\mathrm{MCMC}} \leq 0.001^{* * *},<0.01^{* *}, \leq 0.05^{*},>0.05$ n.s. 
Table S.6.10. Fixed and random effects in explaining variation in $\mathrm{F}_{2}$-offspring cross-sectional body area $\left(\mathrm{mm}^{2}\right)$ estimated using the MCMCglmm package (Hadfield, 2010). The default normal priors were used for the fixed effects with the expected value of 0 and variance $10^{12}$. Inverse-Wishart priors were used for the variances with the expected value of 1 and degree of belief of 0.002 . The number of iterations was $1,500,000$, with a burnin of 500,000 and a thinning of 1000 .

\begin{tabular}{rcccccc}
\hline \multicolumn{7}{c}{ F $_{2}$-offspring cross-sectional body area } \\
\hline \multicolumn{7}{c}{ Fixed effects } \\
intercept & $\beta_{\text {post.mean }}$ & $2.5 \% \mathrm{Cl}$ & $97.5 \% \mathrm{Cl}$ & eff.samp & $P_{\mathrm{MCMC}}$ & Sig. \\
LF treatment & -0.156 & -0.224 & 4.572 & 1000.000 & 0.001 & $* * *$ \\
day 2 after birth & 0.315 & 0.110 & 0.486 & 1413.887 & 0.001 & $* * *$ \\
day 7 after birth & 0.333 & 0.137 & 0.539 & 1233.689 & 0.001 & $* * *$ \\
LF treatment $\times$ day 2 after birth & -0.094 & -0.361 & 0.184 & 1289.874 & 0.482 & n.s. \\
LF treatment $\times$ day 7 after birth & 0.164 & -0.132 & 0.432 & 1000.000 & 0.268 & n.s. \\
\hline
\end{tabular}

\begin{tabular}{lcccc}
\hline \multicolumn{5}{c}{ Random effects } \\
\hline & $\sigma_{\text {post.mean }}^{2}$ & $2.5 \% \mathrm{Cl}$ & $97.5 \% \mathrm{Cl}$ & eff.samp \\
\hline offspring identity & 0.2926 & 0.1882 & 0.4028 & 1000.0000 \\
residual & 0.1288 & 0.0932 & 0.1653 & 1000.0000 \\
\hline
\end{tabular}

$\beta_{\text {post.mean }}$ : posterior mean of regression coefficient; $\mathrm{Cl}$ : confidence interval; eff.samp: effective sample size; $P_{\mathrm{MCMC}}$ : posterior bayesian $p$-value; LF: low food; significant codes: $P_{\mathrm{MCMC}} \leq 0.001^{* * *},<0.01^{* *}, \leq 0.05^{*},>0.05$ n.s. 


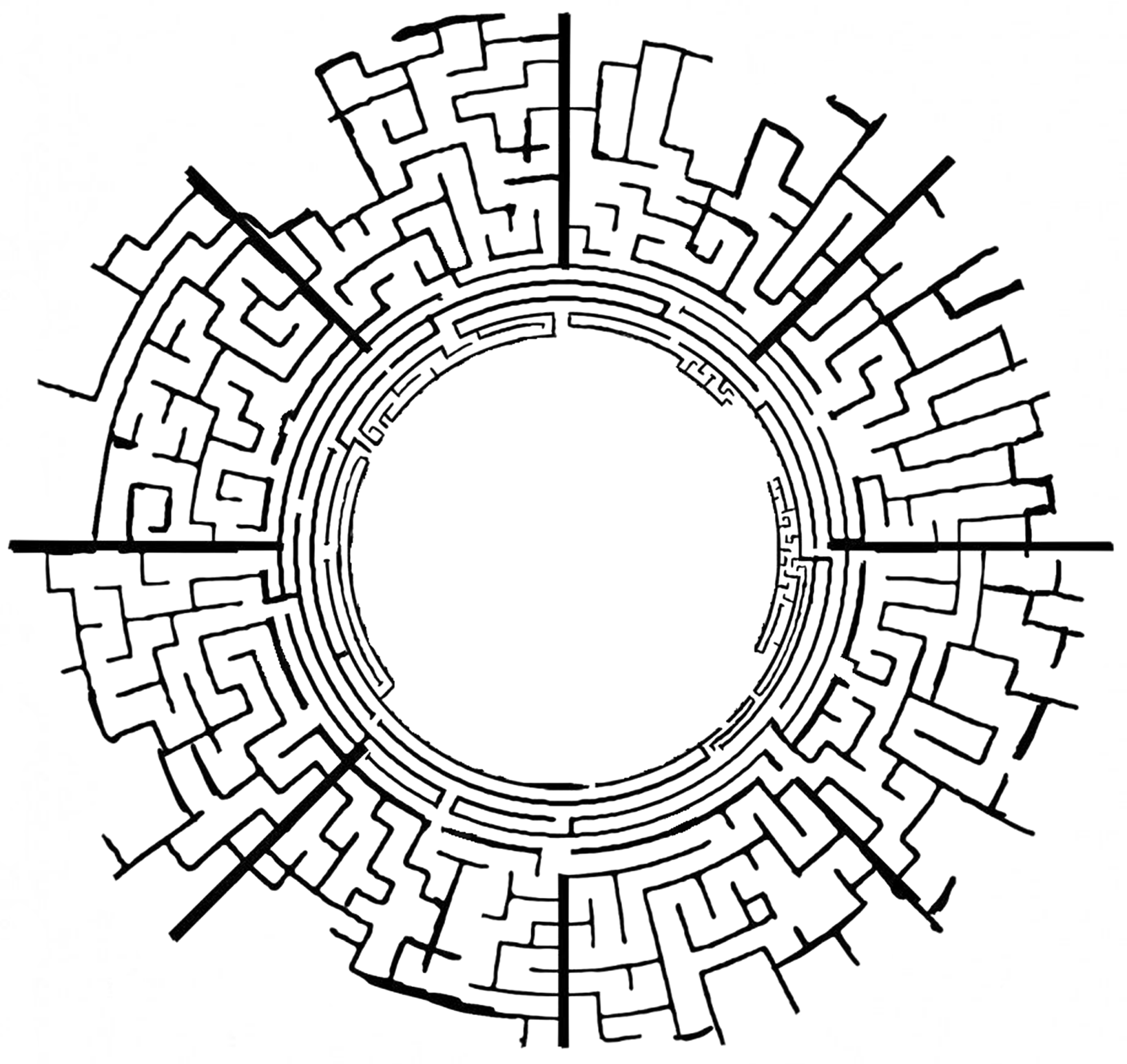




\section{General discussion}

Understanding the origin and elaboration of complex traits is of fundamental interest to scientists and non-scientists alike. The placenta is an excellent example of a complex lifehistory trait that has evolved repeatedly - and continues to evolve to date - throughout the animal kingdom in live-bearing fish (Wourms, 1981), sharks (Hamlett et al., 1993), reptiles (Blackburn, 2015), amphibians (Wake, 2015), mammals (Wooding \& Burton, 2008), and a variety of invertebrates (Ostrovsky et al., 2016). This repeated evolution and ongoing elaboration appears to point towards an adaptive advantage to specific environmental conditions; however, this potential benefit is currently insufficiently understood.

In this thesis, I attempt to contribute to a better understanding of the causes and consequences of placental evolution by drawing on insights from the live-bearing fish family Poeciliidae. Specifically, I aim to shed light on the obscure origin of the placenta. Furthermore, I examine the impacts of environmental stressors during pregnancy on early life development of offspring, as well as the consequences of acquiring a placenta for a species' ecology.

For this, I first quantified biologically relevant sources of variation in placentation to identify potential selective agents that ultimately drive the evolution of the placenta. Specifically, I studied natural populations of the highly placental live-bearing fish species Poeciliopsis retropinna from Costa Rica to examine maternal condition-, size- and/or agerelated offspring provisioning, and hence maternal sources of placental variation (Chapter 2 ), as well as to detect ecological drivers that underlie the ongoing evolution of placentas (Chapter 3). In the latter, I related inter-population variation in the degree of placentation to predation risk in streams to test a key prediction of the locomotor cost hypothesis, which is that placentation evolves in 'performance-demanding' (high predation) environments by reducing the reproductive burden of females during pregnancy, and hence, improving swimming performance. If true, I additionally predicted placental species to inhabit higher 'performance-demanding' (micro)habitats (e.g. high-flow velocity) compared to closely related non-placental species. I empirically tested this idea in five sympatric live-bearing fish species (family Poeciliidae) in Costa Rica that differ in the absence/presence of placentation (Chapter 4). In Chapter 5 and 6, I examined the potential risks of the intimate link the placenta forms between the mother and fetus when exposed to adverse environmental conditions (Chapter 5: maternal parasite infestation in 
P. retropinna; Chapter 6: maternal malnutrition in Phalloptychus januarius).

In the following discussion, I put the findings of my $\mathrm{PhD}$ research into a wider context. First, I restate the different hypotheses for the evolution of the placenta together with the currently available evidence for each (Section 7.1). Second, because superfetation is commonly found in Poeciliidae and thought to co-evolve with the evolution of the placenta, I developed an R-package to simulate and graphically illustrate the potential advantages of having superfetation, as well as both, superfetation and placentation (Section 7.2, Supplementary Methods S.7.1.1). The simulation can be seen as a first step on which future, empirical studies can build upon to assess the costs of locomotion as a function of superfetation and placentation. The R-package and instructions for the installation can be found here: https://github.com/andreshagmayer/ReproductiveBurden.git. Third, I discuss the potential disadvantages of having a placenta in response to various adverse environmental conditions (Section 7.3). Fourth, I address the key questions arising from this thesis, and discuss the steps that are required to answer these questions, as well as the challenges in doing so (Section 7.4). Finally, I end with the concluding remarks from this thesis (Section 7.5).

\subsection{What drives the evolution of the placenta?}

To date, two adaptive hypotheses (termed the resource allocation and locomotor cost hypotheses) provide potential explanations for why the placenta evolves. Both hypotheses treat the placenta as a life-history adaptation that evolves in response to ecological selection pressures (Thibault \& Schultz, 1978; Trexler \& DeAngelis, 2003). Furthermore, both hypotheses posit that the ancestral state is for all nutrients to be prepackaged in the form of egg yolk (lecithotrophy) and that the derived state is for nutrients to be supplied to embryos throughout pregnancy via a placenta (placentotrophy). This is presumably achieved via a gradual shift in the timing of nutrient provisioning from pre- to post-fertilization. If true, then the evolution of the placenta should coincide with a reduction in egg mass at fertilization without affecting the size and quality of offspring at birth (Reznick et al., 2002; Trexler \& DeAngelis, 2003; Pollux et al., 2009).

\subsubsection{Locomotor cost hypothesis}

The locomotor cost hypothesis (Thibault \& Schultz, 1978; Pollux et al., 2009; Pires et al., 2011) postulates that the placenta evolves to offset some of the locomotor costs associated with a live-bearing mode of reproduction. The physical and physiological burden of pregnancy negatively affects a female's locomotor performance in a broad range of livebearing animals (Seigel et al., 1987; Plaut, 2002; Noren et al., 2011; Fleuren et al., 2019). The evolution of the placenta should allow females to attain higher fitness, because the production of smaller eggs at fertilization reduces a female's reproductive burden during 
pregnancy. This improves body streamlining and locomotor performance, notably without sacrificing reproductive output. The presumed benefit of a higher degree of placentation is that the improved swimming performance offers a selective advantage to females in 'performance-demanding' (e.g. high-flow velocity or high-predation) environments because it enhances survival probability (Pollux et al., 2009; Pires et al., 2011).

The findings from Chapter 3 support this idea. Specifically, we used the Matrotrophy Index (MI), calculated as the ratio of offspring mass at birth to egg mass at fertilization, to quantify the degree of placentation among 28 natural populations of the highly placental Poeciliopsis retropinna. Comparisons of the degree of placentation among natural populations are of particular interest, because they may provide insights into the evolutionary processes that drive the elaboration of placentas at a macro-evolutionary level (Hansen \& Martins, 1996; Arnold et al., 2001; Reznick \& Ricklefs, 2009; Rolland et al., 2018). We showed a more than two-fold range in the estimated degree of placentation among the populations, which was correlated with predation risk in streams. Particularly, we found that female $P$. retropinna that co-occur with piscivorous predator species produce smaller eggs at fertilization, while giving birth to an equal number, size, and quality of offspring. The life-history adjustments lead to a significantly higher degree of placentation and a lower reproductive allotment in females that have to cope with high predation risk. To date, only few other studies have evaluated inter-population variation in the degree of placentation (e.g. Schrader \& Travis 2005; Pires et al. 2007; Gorini-Pacheco et al. 2017). However, these did not examine (or used insufficient replicates to make reliable inferences about) the ecological factors that may have driven differences in the degree of placentation among populations.

A number of other recent studies lend additional compelling credence to the findings from Chapter 3 by showing that a lower reproductive allotment can indeed convey a benefit to females by improving their locomotor performance during pregnancy. Fleuren et al. (2018) showed that placental species are more slender at the beginning of pregnancy compared to non-placental ones and that, consistent with the locomotor cost hypothesis (Pollux et al., 2009), this morphological advantage of the placenta diminishes over the course of gestation. A lower reproductive allotment and streamlined body shape are associated with a reduced drag on the female body (Quicazan-Rubio et al., 2019), higher sustained swimming performance (Plaut, 2002), improved fast-start escape response (Ghalambor et al., 2004; Fleuren et al., 2019), and enhanced survival probability (Walker et al., 2005; Plath et al., 2011; Laidlaw et al., 2014). The observed increased degree of placentation and associated decreased reproductive allotment in high predation populations may thus lead to an enhanced predator escape performance. In conclusion, our results provide the first compelling evidence in support of the locomotor cost hypothesis by showing that an increase in the degree of placentation can offer a selective advantage in high 'performancedemanding' (i.e. high predation) environments (Hagmayer et al., 2020c). 


\subsubsection{Resource allocation hypothesis}

The resource allocation hypothesis predicts placentas will enhance fecundity in stable, high-resource environments (Trexler \& DeAngelis, 2003). Placental females produce smaller, less expensive eggs, and transfer nutrients to the developing embryos throughout gestation (Wourms, 1981). By reducing the initial cost of eggs, placental females of equal size and body condition have the potential to fertilize more eggs and gestate more embryos. If sufficient nutrients are available to provision all developing embryos, placental provisioning may increase maternal fecundity (Trexler \& DeAngelis, 2003). If food is scarce or fluctuating, females can provision young with stored maternal fat reserves or abort and resorb them and provision only as many as they can fully nourish (Trexler, 1997; Trexler \& DeAngelis, 2003). However, if placental females are unable to abort embryos and recycle the invested energy, the benefits of placentation are greatly reduced. Trexler \& DeAngelis (2003) therefore argued that the ability to selectively abort embryos and recycle this investment, should resource conditions suddenly deteriorate, is a crucial preadaptation for the evolution of placentation.

Several findings appear to tentatively argue against the resource allocation hypothesis. First, prior studies in four independent placental lineages have not shown that poeciliid fish are able to abort embryos in response to sudden adverse resource conditions (Reznick et al., 1996b; Banet \& Reznick, 2008; Banet et al., 2010; Pollux \& Reznick, 2011). Second, available evidence suggests that maternal body fat might not fully buffer placental females against unfavorable environmental conditions (Pollux \& Reznick, 2011). The placental fish species Heterandria formosa, Poeciliopsis turneri, P. prolifica, and Phalloptychus januarius all responded to low food conditions under laboratory conditions by producing smaller offspring at birth with less fat reserves (Reznick et al., 1996b; Pollux \& Reznick, 2011). In concurrence with these laboratory studies, we showed in Chapter 2, under natural conditions in field-collected specimens of Poeciliopsis retropinna, that decreasing maternal body condition (i.e. proportion body fat) is negatively associated with offspring size and body condition at birth. Finally, if female fat storage would serve to buffer placental species from fluctuating resource conditions, then abortion incidence should increase when food conditions become unfavorable and maternal fat reserves insufficient to fully buffer females during gestation. In other words, better-conditioned females (those carrying larger fat reserves) should be less likely to abort embryos. Contrary to this expectation, we found in Chapter 3 an increase in the abortion incidence with a higher proportion of maternal body fat: better-conditioned females were more likely to abort embryos. The mechanisms behind this higher rate of embryo abortion in better-conditioned female $P$. retropinna are currently unclear and require further investigation.

\subsubsection{Other drivers of placental evolution: conflict hypothesis}

Both the resource allocation and locomotor cost hypothesis provide potential adaptive explanations for why the placenta evolves. In contrast to these adaptive hypotheses, 
the conflict hypothesis posits that the placenta evolves as a non-adaptive by-product of a self-reinforcing arms race between mothers and gestating offspring over control of resource transfer (Haig, 1993; Zeh \& Zeh, 2000; Crespi \& Semeniuk, 2004; Pollux et al., 2009).

Specifically, the prolonged physical contact between mother and offspring in live-bearing species creates the opportunity for embryos to influence maternal nutrient provisioning (Crespi \& Semeniuk, 2004). A conflict of interest between parents and offspring will arise when they are not genetically identical and when both can actively influence the transfer of resources (Trivers, 1974). A mother who has an equal degree of relatedness to any of her offspring $\left(r=\frac{1}{2}\right)$, will benefit most by providing sufficient nutrients to as many offspring as possible to ensure their survival. By contrast, an offspring will demand more than merely sufficient resources, as any given offspring is more closely related to itself $(r=1)$ than to its full- $\left(r=\frac{1}{2}\right)$ or half-siblings $\left(r=\frac{1}{4}\right)$ (Trivers, 1974). This conflict of interest between mother and developing offspring over the degree of maternal provisioning is hypothesized to give rise to antagonistic co-evolution, driving the evolution of more complex placentas (Haig, 1993; Crespi \& Semeniuk, 2004).

A current study provides tentative support for the conflict hypothesis. Using an extensive data set consisting of 9,432 individuals belonging to 160 poeciliid species, Furness et al. (sub) failed to find an association between the degree of placentation and climate/habitat variables, which would have been indicative of an adaptation to ecological selection pressures (i.e. adaptive hypotheses). Though, they emphasize the limitations of the used climate variables as proxies of the selective environment (Furness et al., sub). In addition, other studies on eutherian and metatherian mammals support the idea that many features of the placenta have evolved as antagonistic co-evolution between maternal and fetal tissues struggling over resources (Haig, 1993; Crespi \& Semeniuk, 2004; Elliot \& Crespi, 2006).

\subsubsection{Conclusion}

The three main hypotheses for the evolution of the placenta outlined in this chapter are not mutually exclusive. Each is likely to have played a role at different stages of the evolutionary process. Whereas its initial evolution might have been driven by ecological selection pressures, its subsequent rapid diversification may have been accelerated by conflict-driven antagonistic co-evolution (Pollux et al., 2009).

\subsection{Interplay between placentation and superfetation}

Superfetation is the ability to carry several broods at different developmental stages (Turner, 1937). This reproductive adaptation is commonly found in Poeciliidae and thought to co-evolve with the evolution of the placenta (Thibault \& Schultz, 1978; Reznick \& Miles, 1989; Reznick et al., 2007; Pollux et al., 2009). The presence of the placenta and superfetation are both thought to reduce the reproductive burden, enhance body 
streamlining, and thus improve the locomotor performance of females during pregnancy (Thibault \& Schultz, 1978; Pollux et al., 2009; Zúñiga-Vega et al., 2010; Pires et al., 2011; Fleuren et al., 2018, 2019). Therefore, placentation and superfetation are likely to interact in shaping the reproductive burden, and hence integrating the effects of superfetation is fundamental for studying the evolution of the placenta. However, a numerical quantification of the effects of superfetation, as well as the interaction between superfetation and placentation on the reproductive burden is still lacking in literature. Therefore, I developed an R-package to simulate and graphically illustrate the potential advantages of having superfetation, as well as both, superfetation and placentation. The simulation can be seen as a first step on which future, empirical studies can build upon to assess the costs of locomotion as a function of superfetation and placentation.

\subsubsection{Simulating reproductive allotment}

I used the reproductive allotment (i.e. the mass allocated to developing offspring) as a proxy for a female's reproductive burden during pregnancy. A female's reproductive allotment can be simulated through time by using the estimates of several individual maternal life-history traits (i.e. degree of superfetation, brood size, egg mass at fertilization, and offspring mass at birth). The reproductive allotment can be defined as:

$$
r_{t}=\sum_{i=1}^{s} b_{i, t}=\sum_{i=1}^{s} e_{i, t} l_{i, t},
$$

where $r_{t}$ corresponds to the reproductive allotment of a hypothetical female at time $t, s$ to the degree of superfetation (i.e. the number of broods at different developmental stages), $\sum_{i=1}^{s} b_{i, t}$ to the sum of all brood masses $(b)$ within the female at time $t$ and $\sum_{i=1}^{s} e_{i, t} l_{i, t}$ to the sum of the product between the mean embryo mass $(e)$ and brood size $(l)$ of all broods within the female at time $t$. The mean embryo mass $(e)$ is estimated as the exponential relationship between embryo mass and the developmental stage of embryos $(d)$ at time $t$ in the following linear model (Chapter 2):

$$
\log \left(e_{i, t}\right)=\alpha+\beta d_{i, t},
$$

where

$$
\alpha=\log (f) \quad \text { and } \quad \beta=\frac{\log (o)-\log (f)}{\text { gestation time }} .
$$

'Gestation time' corresponds to $n$ developmental stages, and $f$ and $o$ to egg mass at fertilization and offspring mass at birth, respectively. Consequently, in the simulation I assume that the degree of superfetation, brood size, egg mass at fertilization, and offspring mass at birth are all constant for a given female. Note that because the exact 
relationship between the embryo developmental stage and time is unknown, the simulation predicts the reproductive allotment as a function of 'developmental stage' as a proxy for time. The simulation can then be used to predict the expected reproductive allotment of a hypothetical female at time $t$ (Fig. 7.1). Moreover, the simulation can be used to shed light on the consequences of adjusting individual maternal life-history traits for the reproductive allotment of a female during gestation. In other words, we can examine the interactive effect of superfetation and placentation in shaping a female's reproductive allotment.

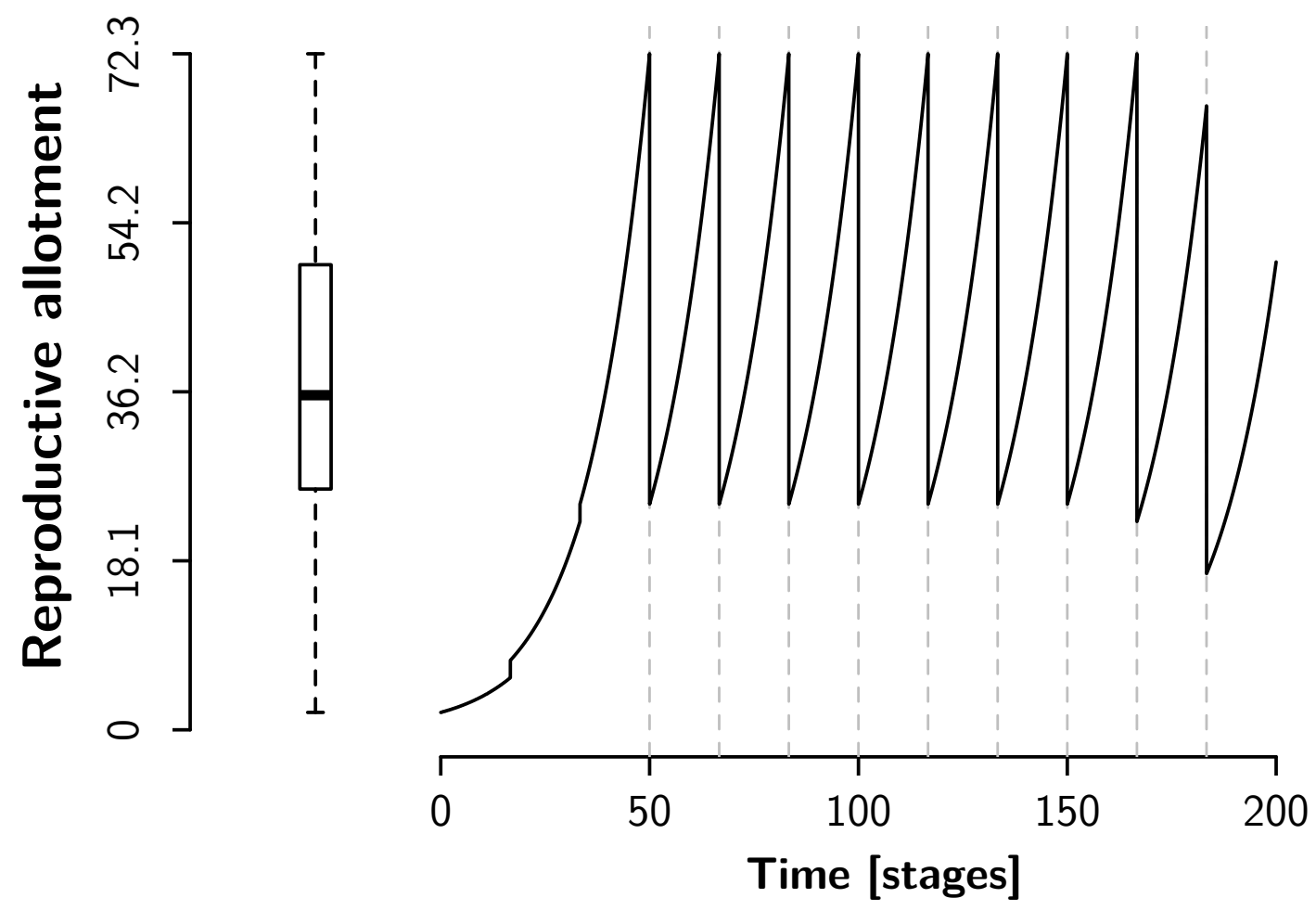

Fig. 7.1. Simulated reproductive allotment over time (developmental stages) for a hypothetical female Poeciliopsis retropinna with population-average life-history traits (i.e. degree of superfetation: 3; brood size: 6 ; egg dry mass at fertilization: $0.31 \mathrm{mg}$; offspring dry mass at birth: $8.34 \mathrm{mg}$ ). The histogram indicates the median and range of the reproductive allotment that is experienced by the female over time. Dashed vertical lines mark the parturition of a brood.

\subsubsection{What is the adaptive advantage of having superfetation?}

Species with superfetation tend to produce smaller broods, but do so more often (Thibault \& Schultz, 1978). By spreading reproduction more evenly over time, superfetation is assumed to reduce the peak reproductive burden of a female during pregnancy, leading to a more slender body shape without affecting a female's fecundity (Downhower \& Brown, 1975; Thibault \& Schultz, 1978). Therefore, superfetation is hypothesized to be favored in environments where a streamlined body is required (e.g. high-flow velocity or 
high-predation localities) or where resources are scarce, and hence reproduction costly (Downhower \& Brown, 1975; Thibault \& Schultz, 1978; Zúñiga-Vega et al., 2010).

Another hypothesis proposes superfetation to increase the rate of offspring production (Burley, 1980; Zúñiga-Vega et al., 2010). Specifically, the overlapping of broods increases the total number of offspring produced per unit time, or in other words, decreases the time required to produce a particular number of offspring. Increasing the rate of offspring production is likely advantageous in environments where adult mortality rate is high relative to that of offspring (Gadgil \& Bossert, 1970; Roff, 1992). Guppies, that lack the ability for superfetation, were shown to deal with increased adult mortality rates by maturing at an earlier age and devoting more resources to reproduction (Reznick et al., 1996a). Superfetation could have evolved as another mechanism to deal with an intense and unpredictable adult survival probability (Burley, 1980; Travis et al., 1987; ZúñigaVega et al., 2010).

Several of the above-mentioned predictions are confirmed by simulating the reproductive allotment:

A. Increasing the degree of superfetation indeed decreases the peak reproductive allotment of a female during pregnancy. However, this is only true if brood size is reduced accordingly. For instance, to increase the degree of superfetation from two to six overlapping broods (black arrow in Fig. 7.2a), a hypothetical placental female with a Matrotrophy Index of 25 (egg mass: $0.4 \mathrm{mg}$; offspring mass: $10 \mathrm{mg}$ ) has to reduce the brood size from 15 to below ten (blue arrow in Fig. 7.2a) to achieve a peak reproductive allotment that is lower than it was before.

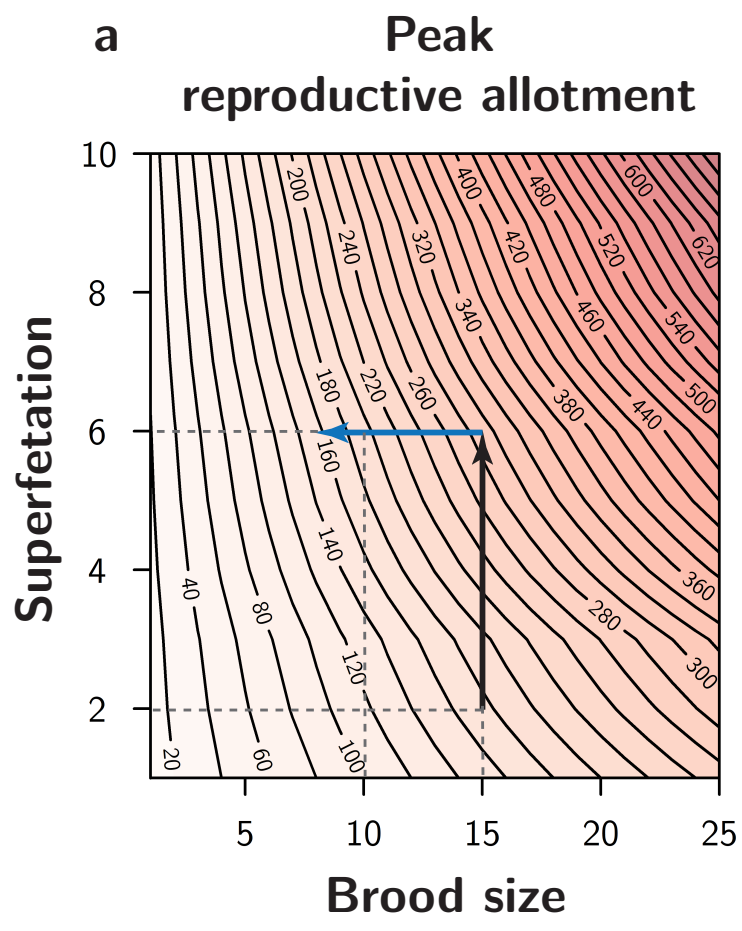

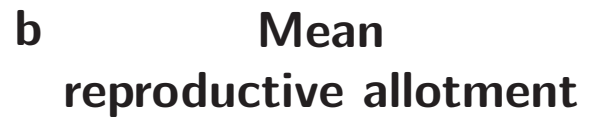

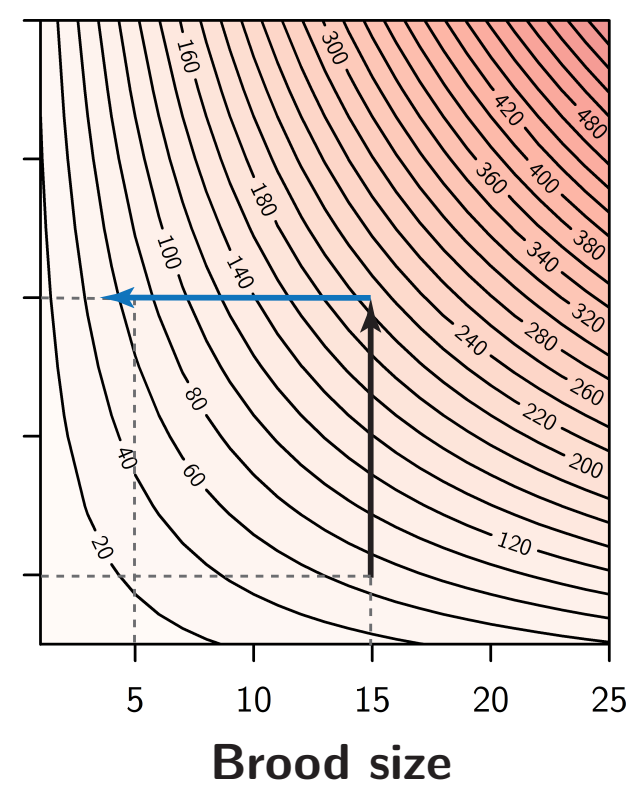


Fig. 7.2. Contour plot for the predicted a peak and $\mathbf{b}$ mean reproductive allotment experienced by a hypothetical placental female with a Matrotrophy Index of 25 (egg mass: $0.4 \mathrm{mg}$; offspring mass: $10 \mathrm{mg}$ ) and a range of different degrees of superfetation and brood sizes (degree of superfetation: 1-10; brood size: 1-25). Note: to increase the degree of superfetation from two to six overlapping broods (black arrow), the brood size has to be reduced from 15 to below ten (blue arrow) to achieve a peak reproductive allotment that is lower than it was before. In contrast, to achieve a mean reproductive allotment that is lower than it was before, the brood size has to be reduced even below five.

Moreover, increasing the degree of superfetation also decreases the mean reproductive allotment. However, brood size must be reduced to a much higher degree, which is likely to impact the overall reproductive output of a female. For instance, to increase the degree of superfetation again from two to six overlapping broods (black arrow in Fig. $7.2 \mathrm{~b}$ ), the same hypothetical female has to reduce the brood size from 15 to below five (blue arrow in Fig. 7.2b) to achieve a mean reproductive allotment that is lower than it was before. In other words, for a fixed reproductive output of a female, increasing the degree of superfetation (if brood size is adjusted accordingly) has a strong negative effect on the peak reproductive allotment, but not, or to a much lesser degree, on the mean reproductive allotment. To test that, I predicted the reproductive allotment for various degrees of superfetation for the same hypothetical female (i.e. MI $=25$ ), while keeping maternal fecundity constant (i.e. increasing the degree of superfetation simultaneously reduces brood size in a way that fecundity remains the same) (Supplementary Methods S.7.1.2). The result shows that for a particular reproductive output, increasing the degree of superfetation strongly decreases the peak reproductive allotment (dark blue curve in Fig. 7.3a), whereas it has no effect on the mean reproductive allotment (light blue curve in Fig. 7.3a).

By strongly reducing the peak reproductive allotment without sacrificing offspring number, superfetation might therefore offer great benefits to females in 'performancedemanding' (e.g. high-flow velocity or high-predation) environments, because it likely enhances streamlining, swimming performance, and hence survival probability (Thibault \& Schultz, 1978; Pollux et al., 2009; Zúñiga-Vega et al., 2010; Pires et al., 2011; Fleuren et al., 2018, 2019).

B. For a fixed lifetime reproductive success and brood size, increasing the degree of superfetation exponentially decreases the reproductive period (i.e. the time required to achieve a particular lifetime reproductive success) (Fig. 7.3b). Thus, superfetation might indeed be an effective mechanism to deal with high and unpredictable adult mortality rates. 
a

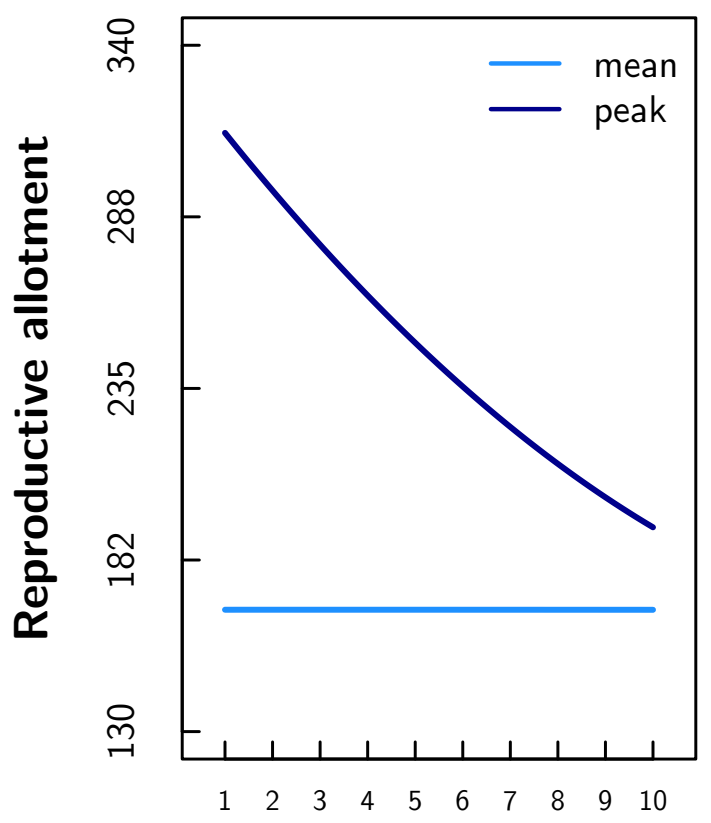

b

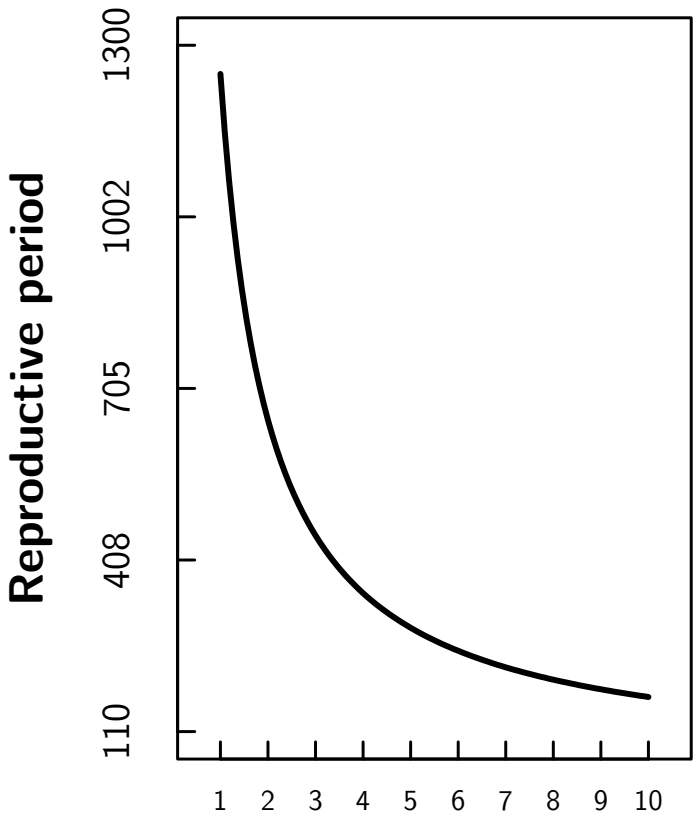

\section{Degree of superfetation}

Fig. 7.3. a Predicted mean (light blue) and peak (dark blue) reproductive allotment for a hypothetical placental female with a Matrotrophy Index of 25 (egg mass: $0.4 \mathrm{mg}$; offspring mass: $10 \mathrm{mg}$ ) and various degrees of superfetation (1-10). Maternal fecundity is kept constant (i.e. an increasing the degree of superfetation simultaneously reduces brood size in a way that fecundity remains the same). b Time required for a hypothetical placental female with a Matrotrophy Index of 25 (egg mass: $0.4 \mathrm{mg}$; offspring mass: $10 \mathrm{mg}$ ), brood size of four embryos, and various degrees of superfetation (1-10), to produce 100 offspring (i.e. reproductive period).

\subsubsection{Are placentation and superfetation reinforcing each other?}

The evolution of superfetation is associated with the evolution of placentation (Pollux et al., 2014; Furness et al., 2019). Therefore, superfetation and placentation most often occur together in the family Poeciliidae (Reznick \& Miles, 1989; Furness et al., 2019). The co-evolution of both traits suggests that one trait may reinforce the effects of the other and vice versa. However, the possible adaptive advantages of the joint evolution of superfetation and placentation are poorly understood.

It has been argued that the shift from pre- to post-fertilization maternal provisioning by means of a placenta, (i) reduces the space required by the embryos in the body cavity, as well as (ii) increases the capacity to provide nutrients for additional broods, as early embryo developmental stages are carried at relatively little cost (Thibault \& Schultz, 1978). This makes it possible to increase the degree of superfetation and hence offspring production rate (Thibault \& Schultz, 1978). In other words, the combination of superfetation and placentation enables these species to produce as many offspring as (or even more 
than) non-placental species without sacrificing offspring size at birth (Pires et al., 2010). Again, this is likely advantageous for instance in environments where adult mortality rate is high relative to that of offspring (Gadgil \& Bossert, 1970; Roff, 1992) or in environments where resources are scarce (Thibault \& Schultz, 1978).

The simulation of the reproductive allotment reveals two potential advantages of combining superfetation with placentation in support of the above-mentioned predictions:

A. Imagine two hypothetical placental species without the ability for superfetation. Both species produce the same number and size of offspring, but differently sized eggs at fertilization: the first species has a Matrotrophy Index (MI) of 25 (egg mass: $0.4 \mathrm{mg}$; offspring mass: $10 \mathrm{mg}$ ) and the second species an MI of 20 (egg mass: $0.5 \mathrm{mg}$; offspring mass: $10 \mathrm{mg}$ ). If we then predict the difference in the expected reproductive allotment between both species throughout pregnancy, we see that the advantage of producing smaller eggs at fertilization becomes zero towards the end of gestation (black curve in Fig. 7.4a). However, if we predict the difference in the expected reproductive allotment between the same two species, but this time with the ability for superfetation, we see that despite producing the same size and number of offspring, superfetation prevents the difference in reproductive allotment at fertilization from becoming zero towards the end of gestation (grey curve in Fig. 7.4a). In other words, superfetation and placentation have a combined effect on diminishing the reproductive burden of females during pregnancy. The simulation herewith corroborates the findings from Chapter 3 based on empirical data for the highly placental Poeciliopsis retropinna with the ability for superfetation (Fig. S.3.8). Specifically, we found that females from high predation populations are associated with a higher degree of placentation compared to low predation females, while producing the same number and size of offspring. Together with the effect of superfetation, this results in a lower reproductive allotment, and hence likely an improved locomotor performance throughout the entire gestation period, rather than only early in pregnancy.

B. Increasing the MI, or degree of placentation, strongly decreases the mean reproductive allotment in females without superfetation (light blue curve in Fig. 7.4b). However, as long as the number and size of the produced offspring remain constant, it does not reduce the peak reproductive allotment (dark blue curve in Fig. 7.4b). By contrast, if placentation is combined with superfetation, an increasing MI results in a strong decrease in both, mean and peak reproductive allotment despite producing the same number and size of offspring (Supplementary Methods S.7.1.2; Fig. 7.4c,d). Yet, superfetation still exclusively affects the peak, but not mean reproductive allotment. Overall, these results suggest that females can reinforce the effects of placentation on the reproductive allotment by additionally overlapping broods (i.e. with superfetation).

These findings are corroborated by empirical results from Chapter 4. If placentation and superfetation evolve in high 'performance-demanding' environments by reducing the reproductive burden of females during pregnancy, and hence, improving locomotor perfor- 
mance (Pollux et al., 2009; Pires et al., 2011), then placental species and/or species with superfetation should be able to utilize higher 'performance-demanding' (micro)habitats compared to closely related non-placental species and/or species that lack the ability for superfetation. We found that during daytime, (i) adults of placental species with superfetation inhabit deep and fast-flowing water, (ii) species that lack both reproductive adaptations are confined to shallow and slow-flowing areas, and (iii) species that lack a placenta but have superfetation occupy an intermediate habitat. Consequently, the coevolution of placentation and superfetation might be favored by the additive effects of both traits on the reproductive burden of females during pregnancy.

a

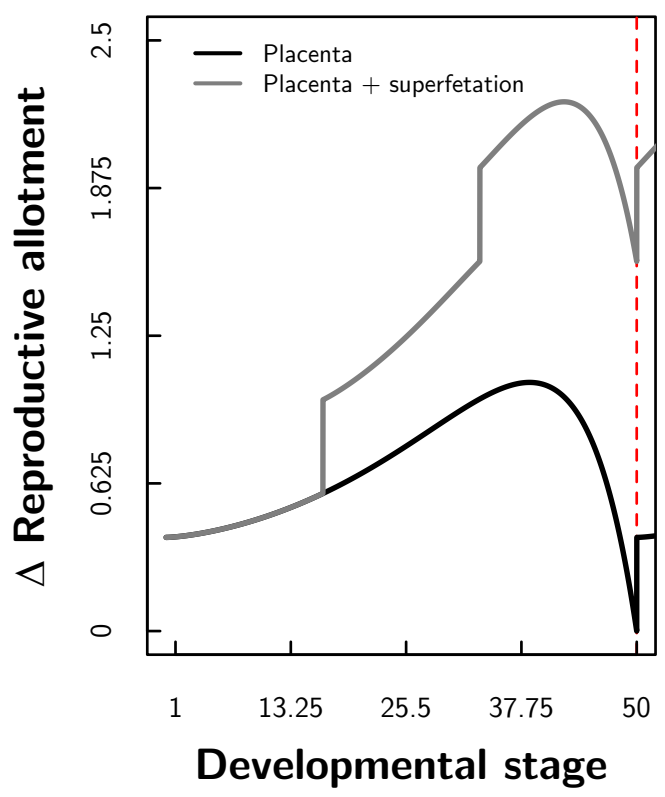

C

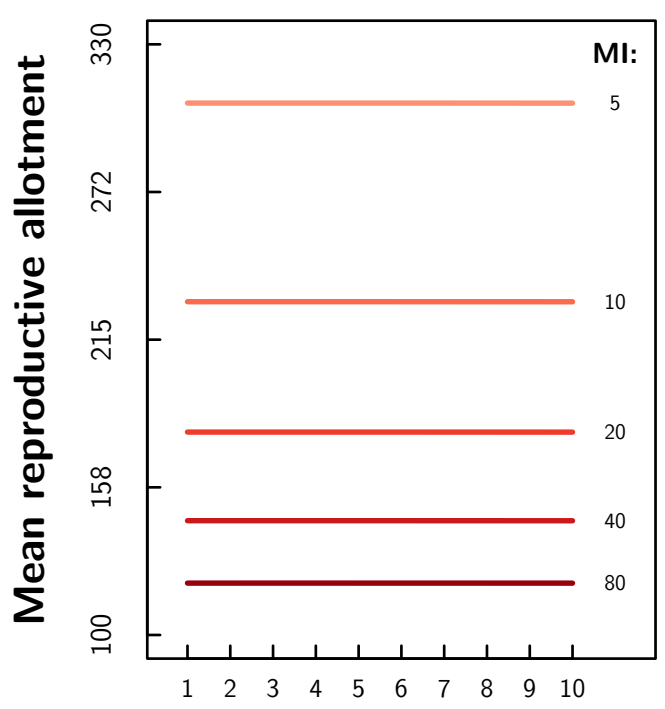

Degree of superfetation b

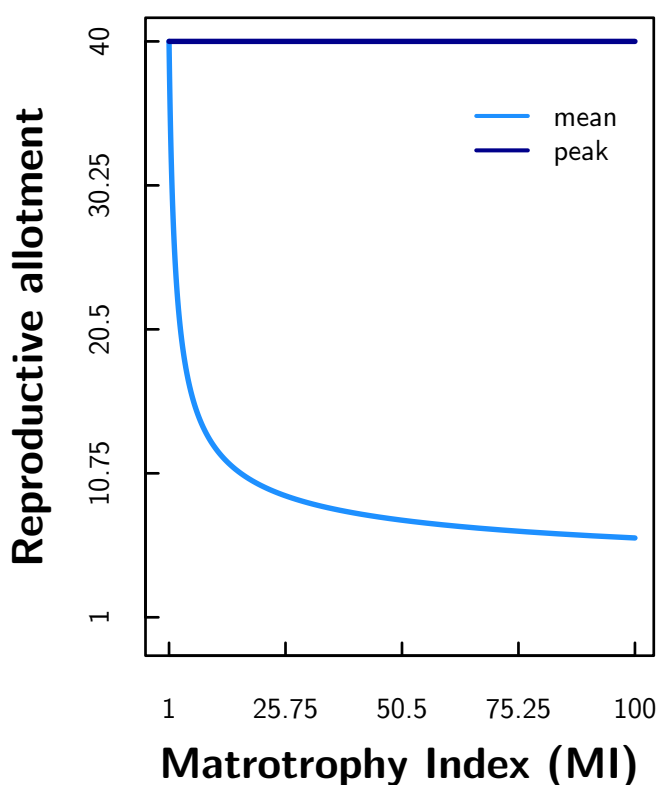

d

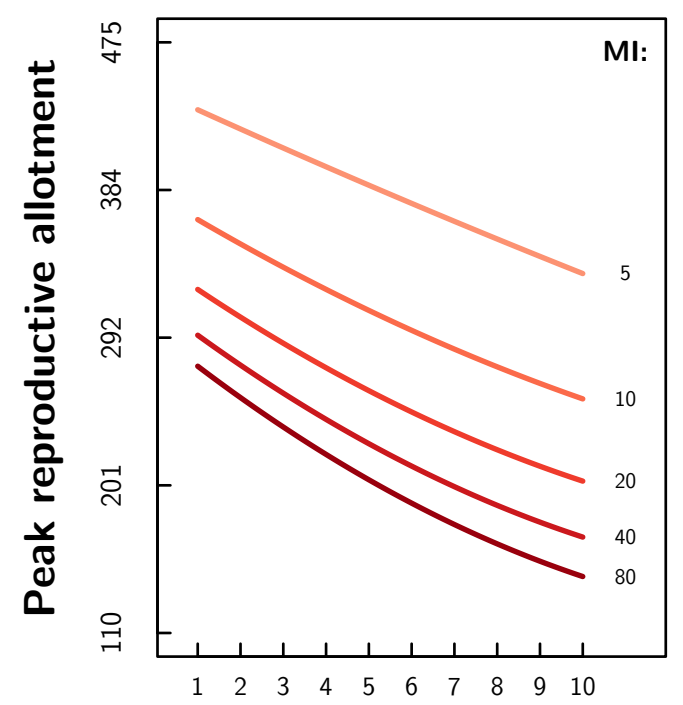

Degree of superfetation 
Fig. 7.4. a Difference in the simulated reproductive allotment throughout pregnancy (developmental stages) between two hypothetical placental species that produce the same number and size of offspring (brood size: 4; offspring mass: $10 \mathrm{mg}$ ), but differently-sized eggs at fertilization (0.4 versus $0.5 \mathrm{mg}$ ). Two scenarios are shown: (i) both species lack the ability for superfetation (black curve), and (ii) both species have the ability to overlap three broods (i.e. degree of superfetation: 3; grey curve). Note: in the latter case, superfetation prevents the difference in reproductive allotment at fertilization from becoming zero towards the end of gestation (dotted red line). b Simulated reproductive allotment as a function of the degree of placentation (i.e. Matrotrophy Index, MI) for a hypothetical female that lack the ability for superfetation (brood size: 4; offspring mass: $10 \mathrm{mg}$ ). Note: an increasing Ml strongly decreases the mean reproductive allotment (light blue curve), however, as long as the number and size of the produced offspring remain constant, it does not reduce the peak reproductive allotment (dark blue curve). c Predicted mean and $\mathbf{d}$ peak reproductive allotment as a function of the degree of superfetation for five hypothetical placental females with constant offspring mass at birth $(10 \mathrm{mg})$, but variable egg mass at fertilization (2, $1,0.5,0.25$, and $0.125 \mathrm{mg}$ ), and thus Ml's $(5,10,20,40$, and 80$)$. Maternal fecundity is kept constant (i.e. an increasing the degree of superfetation simultaneously reduces brood size in a way that fecundity remains the same). Note: if placentation is combined with superfetation, an increasing $\mathrm{MI}$ results in a strong decrease in both, mean and peak reproductive allotment despite producing the same number and size of offspring. By contrast, superfetation only affects the peak, but not mean reproductive allotment.

\subsection{Can having a placenta be disadvantageous?}

The placenta has evolved independently at least nine times in the fish family Poeciliidae (Pollux et al., 2014; Furness et al., 2019), whereas its evolution is at least partly driven by ecological selection pressures (Chapter 3,4). Specifically, having a placenta improves swimming performance, which offers a selective advantage to females in 'performancedemanding' (e.g. high-flow velocity or high-predation) environments because it enhances survival probability (Pollux et al., 2009; Pires et al., 2011). This raises the question why placentotrophy should not also offer benefits in low 'performance-demanding' (i.e. low-flow velocity or low-predation) environments? In other words, is the evolution of the placenta associated with costs, resulting in the fact that the majority of poeciliid species lack a placenta?

Placental species continuously supply their developing embryos with resources (Wourms, 1981; Pollux et al., 2009). As a result, the timing of determining brood size (i.e. number of embryos) and offspring size are decoupled (Reznick et al., 1996b; Pollux \& Reznick, 2011). Whereas brood size is determined prior to fertilization based on current food availability, offspring size is affected by food availability after fertilization, throughout gestation (Reznick et al., 1996b; Pollux \& Reznick, 2011). Consequently, when resource conditions suddenly deteriorate, placental species may not be able to optimally provision their embryos. Because poeciliid fish may be incapable of selectively aborting embryos 
in response to adverse food conditions (Reznick et al., 1996b; Banet \& Reznick, 2008; Banet et al., 2010; Pollux \& Reznick, 2011), and maternal body fat might not fully buffer placental females during gestation (Pollux \& Reznick, 2011), sudden deteriorated resource conditions inevitably result in the production of smaller, worse-conditioned offspring at birth (Reznick et al., 1996b; Pollux \& Reznick, 2011).

Smaller offspring perform worse on fast start-escapes (Dial et al., 2016), presumably decreasing survival probability in environments with high predation risks. Prey-capture abilities immediately after birth also heavily depend on swimming performance. Newborn live-bearing fish are super-precocial, having functional prey-capture abilities at birth and relying on active exogenous feeding after birth (Lankheet et al., 2016). Moreover, they rapidly develop the visuo-motor skills required for prey-capture during the first days after birth and effectively improve their success rate, promoting food uptake and survival (Lankheet et al., 2016). Offspring size thus affects locomotor performance and prey-capturing abilities after birth. Moreover, we showed that maternal food restriction in the placental live-bearing fish species Phalloptychus januarius, leading to a reduced resource allocation to offspring size and body fat at birth, negatively impacts the postnatal development of locomotor performance during feeding, and thus, body condition and likely fitness after birth (Chapter 6). Therefore, placentotrophy in poeciliids is likely a maladaptive strategy in fluctuating resource environments, because sudden reductions in maternal food availability during pregnancy result in smaller offspring with a lower locomotor performance during early life.

Likewise, in Chapter 5 we showed that independent of maternal body fat and size, heavily parasitized females of the placental live-bearing fish species Poeciliopsis retropinna produce smaller and worse-conditioned offspring at birth, while egg size and quality at fertilization remain unaffected. In general, the amount of resources a female can transfer to her developing offspring per unit of time is the result of a balance between maternal energy uptake (via feeding), her own caloric utilization (maintenance) and the amount of excess energy that is subsequently available for reproduction (Stearns, 1992). In parasitic environments, hosts may allocate an increased amount of resources to parasite defense that might otherwise have been used for different functions (Sheldon \& Verhulst, 1996). It is thus possible that parasitized females simply have less energy available that can be used to invest in developing embryos, which results in the production of smaller and worse-conditioned offspring at birth.

Therefore, although having a placenta offers a selective advantage to females in high 'performance-demanding' environments by improving swimming performance, the intimate link the placenta forms between mother and fetus also poses a risk: maternal exposure to adverse environmental conditions (e.g. malnutrition, parasite infestation) is likely to have unfavorable consequences for fetal development. 


\subsection{Perspectives}

Adaptation requires a phenotypic change due to selection that can be passed on to the offspring (Falconer \& Mackay, 1996). Exploring selective forces and the evolutionary potential of traits, therefore, is fundamental to understand how the diversity of traits evolve and hence address a key challenge in evolutionary biology.

In Chapter 3, we related inter-population variation in the degree of placentation to predation risk in streams to identify potential selective agents that ultimately drive the evolution of the placenta. Investigating inter-population variation in traits is of particular interest, because it may provide insights into the evolutionary processes that drive the elaboration of traits at a macro-evolutionary level (Hansen \& Martins, 1996; Arnold et al., 2001; Reznick \& Ricklefs, 2009; Rolland et al., 2018). We then used the estimates of the effects of body shape on locomotor performance and survival probability based on other poeciliid species to predict the expected locomotor performance, and hence survival advantage of having a higher degree of placentation in our study species (i.e. Poeciliopsis retropinna). However, (i) care must be taken when translating the estimates of particular effects to other species. Specific relationships may not be equally valid across different species. Thus, an empirical quantification of the selective forces acting on the degree of placentation in P. retropinna is still lacking. And (ii), although selection acts on phenotypic variation associated with fitness, regardless of the genetic basis of the trait (Lande \& Arnold, 1983), the potential for an adaptive change in response to this selection depends on the relative importance of genetic and environmental variation underlying this phenotypic variation, i.e. on its heritability (Falconer \& Mackay, 1996). However, heritability estimates for the degree of placentation are still lacking in literature. Future studies could therefore try to empirically estimate the amount of heritable variation in the degree of placentation (e.g. by using breeding or artificial selection experiments in the laboratory), as well as to quantify the strength of selection (e.g. in survival experiments) to better understand the ecologically-driven evolution of complex traits such as the placenta.

However, exploring selective forces and the evolutionary potential of placentation (and other complex traits) is strongly hampered by analytical and methodological challenges. As of yet, for instance, no non-invasive method exists to determine the degree of placentation (i.e. Matrotrophy Index, MI) in alive specimens. Currently, the MI is quantified by dissecting a female and by measuring offspring mass at birth and egg mass at fertilization based on the embryos carried at different developmental stages in the body cavity. Alternatively, it is possible to confirm nutrient transfer to offspring using tracers injected, however, its quantification also requires euthanization of the animal (Marsh-Matthews, 2011). Because females need to be dissected in order to determine the MI, it (i) requires a large number of individuals in breeding experiments, and (ii) strongly impedes the quantification of fitness components (e.g. the MI needs to be quantified before a female is eaten during predator-prey interactions to quantify survival). Breeding, artificial selection, and survival experiments in the laboratory would therefore greatly benefit by 
developing non-invasive methods to quantify the MI.

Finally, further work is required on the causes and consequences of embryo abortion during pregnancy. Poeciliid fish commonly abort embryos, however, the underlying mechanisms are currently unclear. Prior studies in four independent placental lineages have not shown that embryo abortion is the consequence of sudden adverse resource conditions (Reznick et al., 1996b; Banet \& Reznick, 2008; Banet et al., 2010; Pollux \& Reznick, 2011). Likewise, we showed that abortion incidence did not correlate with predation risk in streams (Chapter 3) or trematode infestation (Chapter 5). Studying the underlying mechanisms of embryo abortion may identify potential environmental stressors affecting embryo development during pregnancy, and hence, early life development and survival of subsequent generations. In addition, this may help to predict population dynamics with implications for the conservation of placental species.

\subsection{Concluding remarks}

This thesis:

- suggests that an increased degree of placentation by means of increasing postfertilization maternal provisioning is likely a plastic response to increased food availability (Chapter 2);

- argues that an increased degree of placentation by means of reducing pre-fertilization maternal provisioning is likely an adaptive response to high predation risk and high water flow velocity (Chapter 3, 4);

- proposes that the co-evolution of placentation and superfetation might be favored by the additive effects of both traits on the reproductive burden of females during pregnancy (Chapter 4, 7);

- identified an important cost of parasite infestation in placental live-bearing fish, which is a reduced maternal nutrient provisioning to embryos, resulting in smaller size and quality, and presumably lower fitness, of offspring at birth (Chapter 5);

- suggests that placentotrophy in poeciliids is likely a maladaptive strategy in fluctuating resource environments, because sudden reductions in maternal food availability during pregnancy result in smaller offspring with a lower locomotor performance during early life (Chapter 6). 


\section{S.7 Supplementary Information}

\section{S.7.1 Supplementary Methods}

\section{S.7.1.1 R-package 'ReproductiveBurden' (https://github.com/andreshagmayer/ ReproductiveBurden.git)}

This R-package allows to simulate and graphically illustrate the expected reproductive allotment, and hence burden, during pregnancy for a live-bearing species as a function of time and for a specific degree of superfetation, litter size (or brood size), egg mass at fertilization, and offspring mass at birth.

Usage:

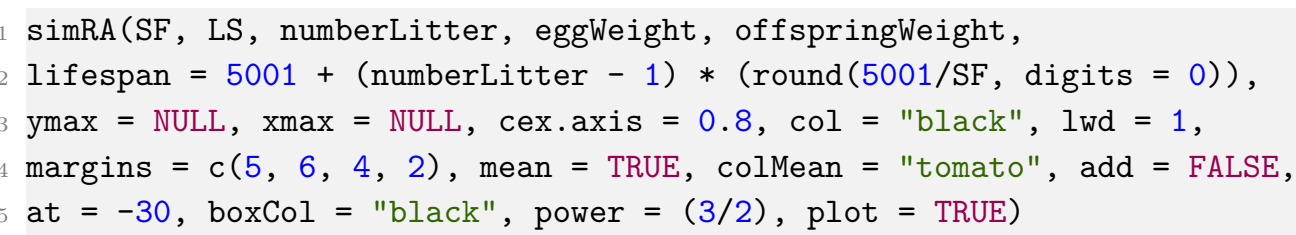

Arguments:

$\begin{array}{ll}\text { SF } & \text { Degree of superfetation; should be } \geq 1 \\ \text { LS } & \text { Number of embryos in a litter } \\ \text { numberLitter } & \text { Number of litters to produce; note: numberLitter = life time reproductive success/brood } \\ & \text { size } \\ \text { eggWeight } & \text { Egg mass at fertilization } \\ \text { offspringWeight } & \text { Offspring mass at birth } \\ \text { lifespan } & \text { Lifespan in developmental stages of embryos (optional); } 50 \text { stages correspond to a com- } \\ & \text { plete gestation cycle and approximately } 30 \text { days in Poeciliidae; default is the time required } \\ & \text { to produce the specified number of litters with the specified degree of superfetation } \\ \text { ymax } & \text { Y-axis limit (optional); default }=\text { max(reproductive burden) } \\ \text { xmax } & \text { X-axis limit (optional); default = max(stages) } \\ \text { cex.axis } & \text { Axes label size (optional) } \\ \text { col } & \text { Curve color (optional); default }=\text { "black" } \\ \text { Iwd } & \text { Line width (optional); default }=1 \\ \text { margins } & \text { Plot margins (optional); default }=\mathrm{c}(5,6,4,2) \\ \text { mean } & \text { If TRUE, the mean will be added to the boxplot (optional) } \\ \text { colMean } & \text { Color of the mean (optional); default }=\text { "tomato" } \\ \text { add } & \text { If TRUE, an additional curve can be added to the plot (optional) } \\ \text { at } & \text { Position of boxplot (optional); default }=-30 \\ \text { boxCol } & \text { Color of the boxplot (optional); default = "black" } \\ \text { power } & \text { Power transformation coefficient for the developmental stage of embryos (optional); de- } \\ & \text { fault }=\frac{3}{2}, \text { which is used for Poeciliopsis retropinna (Hagmayer et al., 2018); if power = } \\ \text { plot } & \text { 1, no power-transformation will be applied (i.e. pure exponential relationship) } \\ & \text { Whether to show the graph (optional); default = TRUE }\end{array}$


Note: this package was designed for matrotrophic species with a Matrotrophy Index $(\mathrm{MI})>1$. There is no guarantee that meaningful results are obtained for species with a $\mathrm{MI}<1$.

Function:

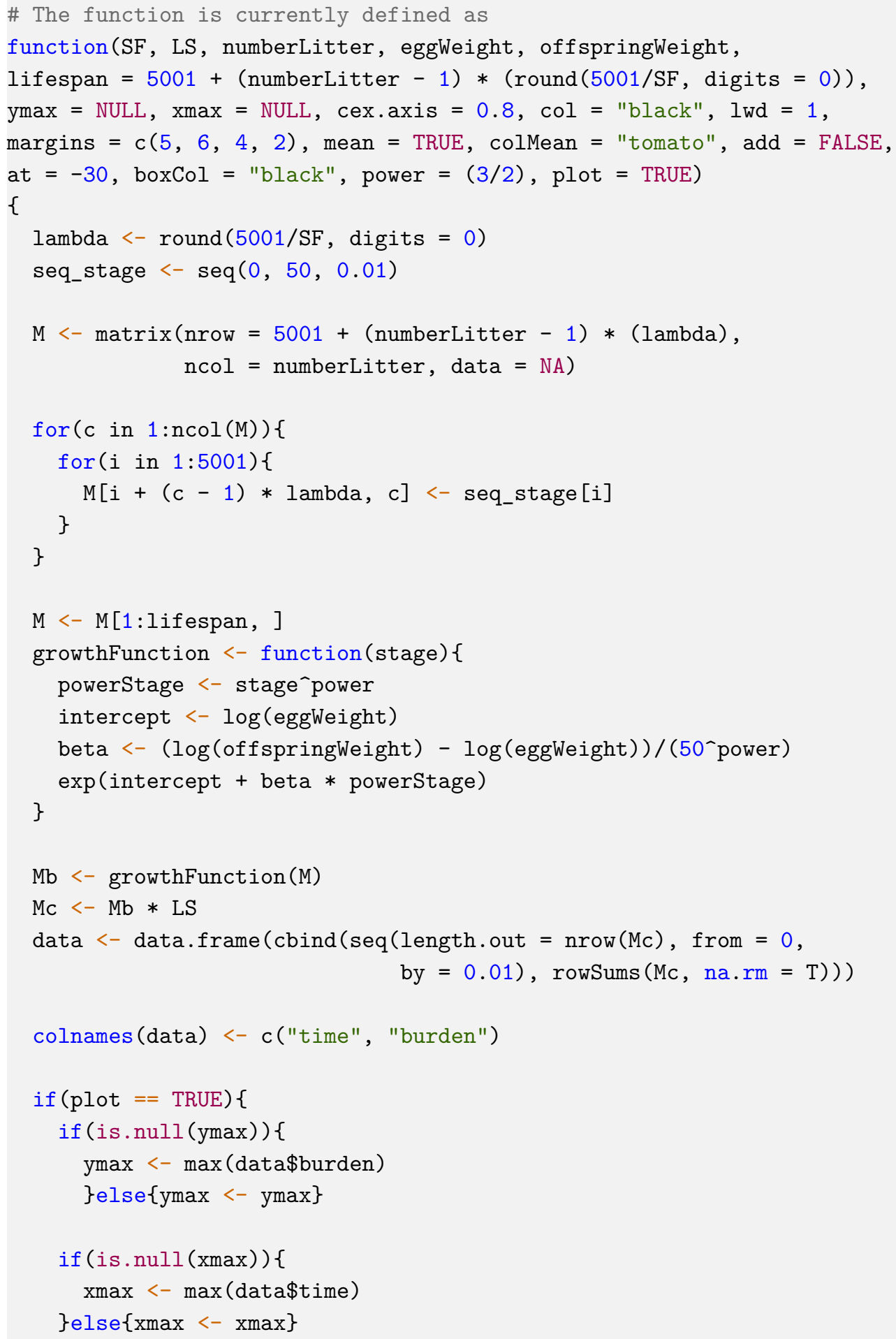




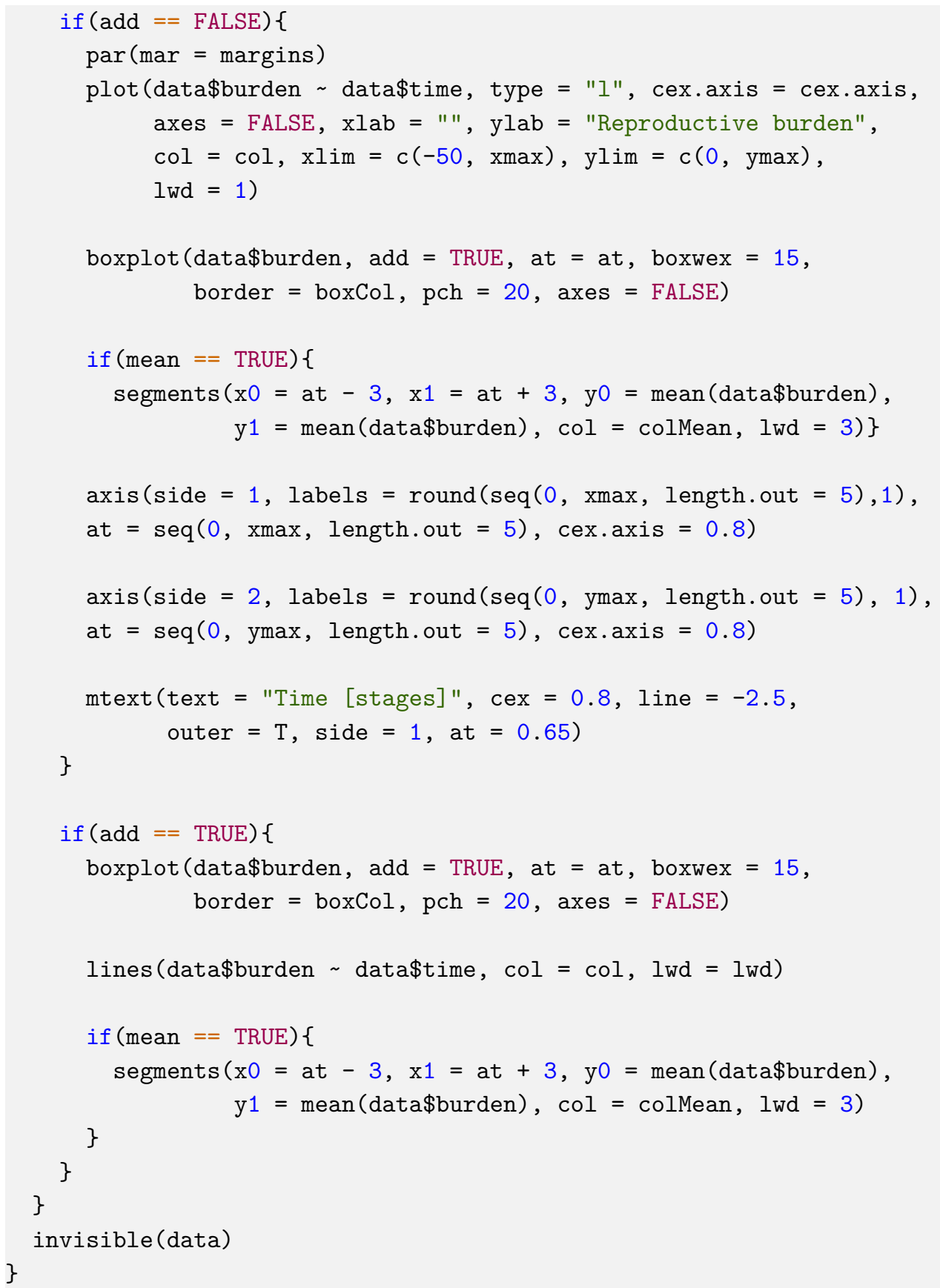

\section{S.7.1.2 Modeling reproductive allotment as a function of superfetation}

Increasing the degree of superfetation decreases the reproductive allotment of a female during pregnancy. However, this is only true if brood size is reduced accordingly. To illustrate the diminishing effect of an increasing degree of superfetation on the reproductive allotment, therefore, brood size has to be adjusted for each degree of superfetation. This can be done statistically by keeping fecundity constant at a given value. Specifically, fecundity during a given reproductive cycle was defined as the product between the degree of superfetation $(s)$ and brood size $(l)$. The mean and peak reproductive allotment $(r)$ 
can then be extracted from the simulations of various parameter combinations, and fitted as a function of superfetation $(s)$ and fecundity $(s \times l)$ in a linear model:

$$
r_{i} \sim N\left(\alpha+\beta_{1} s_{i}+\beta_{2} s_{i}^{2}+\beta_{3} s_{i} \times l_{i}, \sigma^{2}\right) .
$$

Because fecundity is defined as the product between $s$ and $l$, and the model estimates the relationship between $r$ and $s$ by keeping $s \times l$ constant, the effect of an increasing degree of $s$ on $r$ is estimated for an appropriate reduction in $l$. 


\section{Declarations}

\section{Authorship statements}

\section{Affiliation of co-authors}

Chapter 2 to 6 of this thesis were written in collaboration with the following co-authors (sorted in order of appearance and alphabetically per research unit):

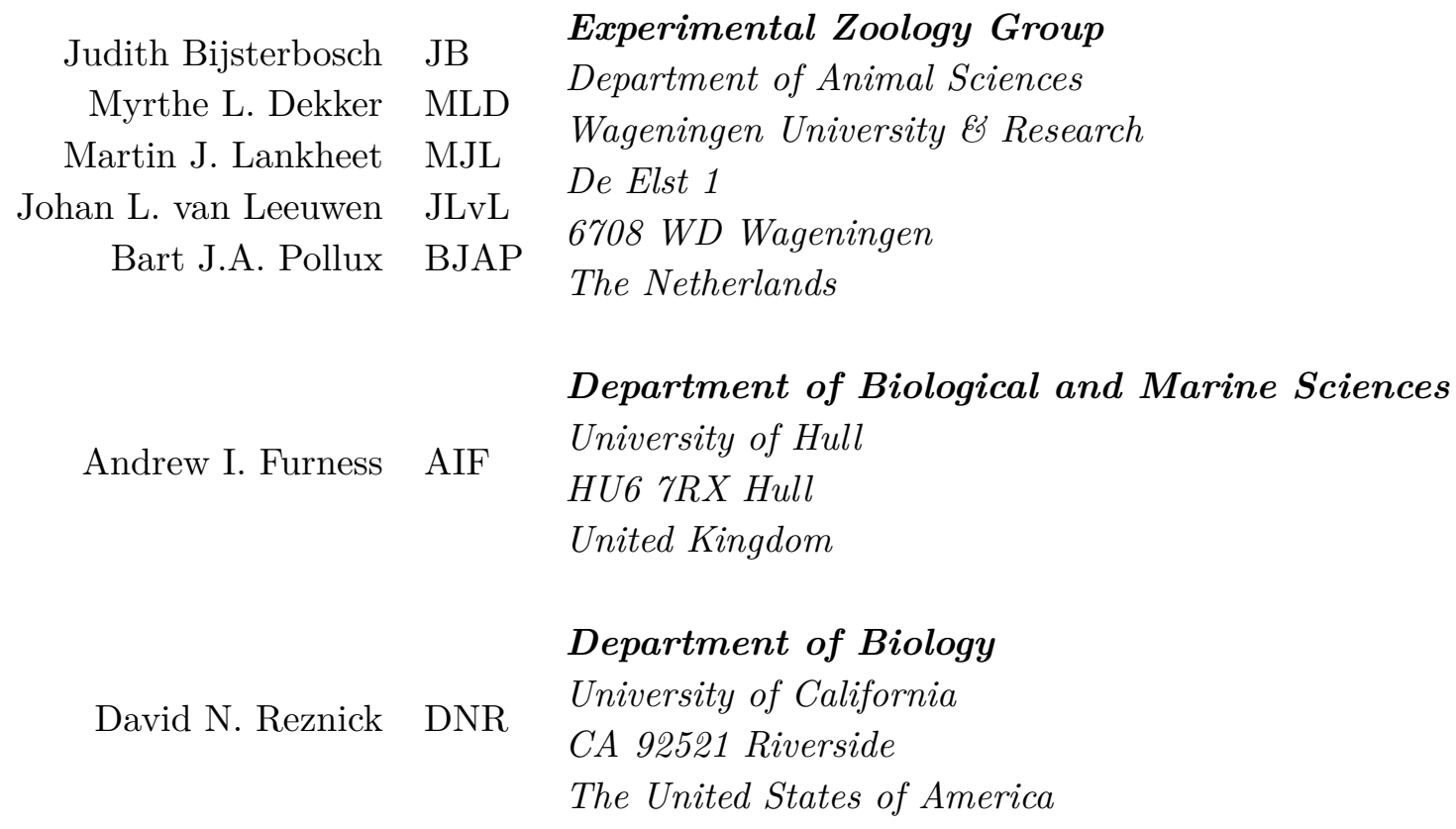

\section{Author contributions}

Andres Hagmayer is abbreviated with AH. For abbreviations of the names of co-authors see the previous section.

\section{Chapter 2}

BJAP and AIF conceived the ideas and planned the fieldwork. BJAP and AIF collected the data. AIF carried out the dissections. AH analyzed the data, wrote the first draft of the manuscript and finalized the manuscript with comments from AIF, DNR, and 
BJAP.

\section{Chapter 3}

AIF and BJAP conceived the project idea. BJAP obtained funding and directed the project. AH, AIF, MLD, and BJAP planned the fieldwork and collected the data. AH and AIF carried out the dissections. AH analyzed the data. AH wrote the first draft of the manuscript supervised by AIF and BJAP. DNR and MLD critically reviewed the initial manuscript and provided helpful input. All authors approved the final manuscript.

\section{Chapter 4}

AH, AIF, and BJAP conceived the ideas, planned the fieldwork, and collected the data. $\mathrm{AH}$ analyzed the data. AH and BJAP wrote the first draft of the manuscript and finalized the manuscript with comments from AIF.

\section{Chapter 5}

AH, AIF, and BJAP conceived the ideas, planned the fieldwork, and collected the data. AH analyzed the data, wrote the first draft of the manuscript and finalized the manuscript with comments from AIF and BJAP.

\section{Chapter 6}

$\mathrm{AH}, \mathrm{MJL}$, and BJAP conceived the ideas and planned the experiment. AH performed the food treatment and MJL built the experimental setup for swimming trials. AH and JB collected the experimental data. MJL and JB developed the software to analyze the swimming trials. AH performed all statistical analyses, wrote the first draft of the manuscript and finalized the manuscript with comments from MJL, JB, JLvL and BJAP.

\section{Acknowledgements}

\section{Chapter 2}

We thank Arturo Angulo and Carlos Garita Alvarado (Universidad de Costa Rica) for their assistance in the field, Zak Zahawi (Estación Biológica Las Cruces y Jardín Botánico Wilson) and Francisco Campos Rivera (Organización para Estudios Tropicales) for their help with the research permits and Rita Vargaz and Ana Rosa Ramírez (Museo de Zoología, Escuela de Biología, Universidad de Costa Rica) for the use of their research facilities. We further thank Yuridia Reynoso (Universitiy of California Riverside) for her help with the dissections and Johan L. van Leeuwen and two anonymous reviewers for commenting on an earlier version of the manuscript. Fishes were collected under collection permit No 190-2012-SINAC. 


\section{Chapter 3}

We thank Arturo Angulo, Carlos Garita Alvarado (Universidad de Costa Rica) and Michael Guernsey (Stanford University) for their assistance in the field, Rebecca Cole (Estación Biológica Las Cruces y Jardín Botánico Wilson), Jimena Paut Brenes, and Francisco Campos Rivera (Organización para Estudios Tropicales) for their help with the research and export permits. We further thank Yuridia Reynoso (University of California Riverside) for her help with the dissections, Karen Leon-Kloosterziel, Henk Schipper, Remco Pieters, and Saskia van Laar (Wageningen University) for providing the laboratory equipment, Timothée Bonnet for statistical advice, Jeffrey Arendt for interesting discussions, and Johan L. van Leeuwen for commenting on an earlier version of the manuscript. Fish were collected under permits No 190-2012-SINAC, No SINAC-CUS-PI-R-005-2017, R-SINAC-ACLAP-011-2018, No INV-ACOSA-024-18, and No SINAC-ACOPAC-D-RES014-2018.

\section{Chapter 4}

We thank Jimena Paut Brenes, Enrique Alonso Castro Fonseca, Rodolfo Quirós Flores, and Rebecca Cole (Organización para Estudios Tropicales) for their help with the research permits. We further thank Johan L. van Leeuwen for commenting on an earlier version of the manuscript. The study was conducted under the research permits No SINAC-CUSPI-R-005-2017, R-SINAC-ACLAP-011-2018, INV-ACOSA-024-18, SINAC-ACOPAC-DRES-014-2018, R-SINAC-ACLAP-002-2019, INV-ACOSA-010-19, and SINAC-ACOPACD-RES-012-2019.

\section{Chapter 5}

We thank Rebecca Cole (Estación Biológica Las Cruces y Jardín Botánico Wilson), Jimena Paut Brenes, and Francisco Campos Rivera (Organización para Estudios Tropicales) for their help with the research and export permits. We further thank Karen LeonKloosterziel, Henk Schipper, Remco Pieters, and Saskia van Laar (Wageningen University) for providing laboratory and field equipment. Fish were collected under permits No SINAC-CUS-PI-R-005-2017, R-SINAC-ACLAP-011-2018, No INV-ACOSA-024-18, and No SINAC-ACOPAC-D-RES-014-2018.

\section{Chapter 6}

We thank Naomi Berbee for her help with biomechanical measurements, the staff of Carus-ARF for their expert advice and help with husbandry.

\section{Ethical statements}

\section{Chapter 2 to 5}

Not applicable. 


\section{Chapter 6}

All procedures were approved by the Animal Ethics Committee of Wageningen University and Research (permit number 2018.W-0022.002).

\section{Funding}

\section{Chapter 2}

BJAP was funded by Schure Beijerinck Popping (SBP2012/61) and VIDI (864.14.008) grants from the Netherlands Organization for Scientific Research and AIF by a SICB graduate research grant and NSF Postdoctoral Fellowship in Biology Award 1523666.

\section{Chapter 3}

BJAP was funded by Schure Beijerinck Popping (SBP2012/61) and VIDI (864.14.008) grants from the Netherlands Organization for Scientific Research, AH by an Academy Ecology Fund 2017 (Eco/1710) from the Netherlands Organization for Scientific Research, and AIF by a SICB graduate research grant and NSF Postdoctoral Fellowship in Biology Award 1523666.

\section{Chapter 4}

AH was partly funded by an Academy Ecology Fund 2017 (Eco/1710) from the Netherlands Organization for Scientific Research, BJAP was funded by a VIDI (864.14.008) grant from the Netherlands Organization for Scientific Research, and AIF by a SICB graduate research grant and NSF Postdoctoral Fellowship in Biology Award 1523666.

\section{Chapter 5}

BJAP was funded by Schure Beijerinck Popping (SBP2012/61) and VIDI (864.14.008) grants from the Netherlands Organization for Scientific Research, AH by an Academy Ecology Fund 2017 (Eco/1710) from the Netherlands Organization for Scientific Research, and AIF by a SICB graduate research grant and NSF Postdoctoral Fellowship in Biology Award 1523666.

\section{Chapter 6}

This work was funded by the Netherlands Organization for Scientific Research grants Schure Beijerinck Popping (SBP2012/61) and VIDI (864.14.008) awarded to BJAP.

\section{Conflict of interest}

\section{Chapter 2 to 6}

All authors declare no conflict of interest. 


\section{Data availability}

Chapter 2

Data available from the Dryad Digital Repository: https://doi.org/10.5061/dryad. qt8744c

\section{Chapter 3}

Data available from the Dryad Digital Repository: https://doi.org/10.5061/dryad. cvdncjt16

\section{Chapter 4}

Data will be made available from a public data repository upon publication of the article or from the corresponding author on reasonable request.

\section{Chapter 5}

Data available from the Dryad Digital Repository: https://doi.org/10.5061/dryad. 6hdr7squr

\section{Chapter 6}

Data will be made available from a public data repository upon publication of the article or from the corresponding author on reasonable request. 



\section{References}

1. Abràmoff, M. D., Magalhães, P. J., and Ram, S. J. (2004). Image processing with imageJ. Biophotonics International, 11(7):36-41.

2. Allee, W. C., Emerson, E., Park, O., Park, T., and Schmidt, K. P. (1949). Principles of animal ecology. Philadelphia.

3. Andrews, K. T. and Lanzer, M. (2002). Maternal malaria: Plasmodium falciparum sequestration in the placenta. Parasitology Research, 88(8):715-723.

4. Arnold, S. J., Pfrender, M. E., and Jones, A. G. (2001). The adaptive landscape as a conceptual bridge between micro- and macroevolution. Genetica, 112-113:9-32.

5. Arrington, D. A. and Winemiller, K. O. (2003). Diel changeover in sandbank fish assemblages in a neotropical floodplain river. Journal of Fish Biology, 63(2):442-459.

6. Auer, S. K. (2010). Phenotypic plasticity in adult life-history strategies compensates for a poor start in life in trinidadian guppies (Poecilia reticulata). The American Naturalist, 176(6):818-829.

7. Auer, S. K., Arendt, J. D., Chandramouli, R., and Reznick, D. N. (2010). Juvenile compensatory growth has negative consequences for reproduction in Trinidadian guppies (Poecilia reticulata). Ecology Letters, 13(8):998-1007.

8. Banet, A. I., Au, A. G., and Reznick, D. N. (2010). Is mom in charge? Implications of resource provisioning on the evolution of the placenta. Evolution, 64(11):3172-3182.

9. Banet, A. I. and Reznick, D. N. (2008). Do placental species abort offspring? Testing an assumption of the Trexler-DeAngelis model. Functional Ecology, 22(2):323-331.

10. Banet, A. I., Svendsen, J. C., Eng, K. J., and Reznick, D. N. (2016). Linking reproduction, locomotion, and habitat use in the Trinidadian guppy (Poecilia reticulata). Oecologia, 181(1):8796.

11. Barber, I. and Dingemanse, N. J. (2010). Parasitism and the evolutionary ecology of animal personality. Philosophical Transactions of the Royal Society B: Biological Sciences, 365(1560):40774088.

12. Barber, I., Hoare, D., and Krause, J. (2000). Effects of parasites on fish behaviour: a review and evolutionary perspective. Reviews in Fish Biology and Fisheries, 10(2):131-165.

13. Bashey, F. (2006). Cross-generational environmental effects and the evolution of offspring size in the Trinidadian Guppy Poecilia reticulata. Evolution, 60(2):348-361.

14. Bashey, F. (2008). Competition as a selective mechanism for larger offspring size in guppies. Oikos, 117(1):104-113.

15. Basolo, A. L. and Wagner, W. E. (2004). Covariation between predation risk, body size and fin elaboration in the green swordtail, Xiphophorus helleri. Biological Journal of the Linnean Society, 83(1):87-100. 
16. Bassar, R. D., Auer, S. K., and Reznick, D. N. (2014). Why do placentas evolve? A test of the life-history facilitation hypothesis in two clades in the genus Poeciliopsis representing two independent origins of placentas. Functional Ecology, 28:999-1010.

17. Bates, D. M., Mächler, M., Bolker, B. M., and Walker, S. C. (2015). Fitting linear mixed-effects models using lme4. Journal of Statistical Software, 67(1):1-48.

18. Bell, A. W. (1995). Regulation of organic nutrient metabolism during transition from late pregnancy to early lactation. Journal of Animal Science, 73(9):2804-2819.

19. Berger, J. (1991). Pregnancy incentives, predation constraints and habitat shifts: experimental and field evidence for wild bighorn sheep. Animal Behaviour, 41(1):61-77.

20. Berkeley, S. A., Chapman, C., and Sogard, S. M. (2004). Maternal age as a determinant of larval growth and survival in a marine fish, Sebastes melanops. Ecology, 85(5):1258-1264.

21. Blackburn, D. G. (2015). Evolution of vertebrate viviparity and specializations for fetal nutrition: a quantitative and qualitative analysis. Journal of Morphology, 276(8):961-990.

22. Boggs, C. L. (1992). Resource allocation: exploring connections between foraging and life history. Functional Ecology, 6(5):508-518.

23. Bollens, S. M. and Frost, B. W. (1989). Predator-induced diet vertical migration in a planktonic copepod. Journal of Plankton Research, 11(5):1047-1065.

24. Bollens, S. M., Frost, B. W., and Lin, T. S. (1992). Recruitment, growth, and diel vertical migration of Euphausia pacifica in a temperate fiord. Marine Biology, 114:219-228.

25. Bolnick, D. I., Svanback, R., Araujo, M. S., and Persson, L. (2007). Comparative support for the niche variation hypothesis that more generalized populations also are more heterogeneous. Proceedings of the National Academy of Sciences, 104(24):10075-10079.

26. Bolnick, D. I., Svanbäck, R., Fordyce, J. A., Yang, L. H., Davis, J. M., Hulsey, C. D., and Forister, M. L. (2003). The ecology of individuals: incidence and implications of individual specialization. The American Naturalist, 161(1):1-28.

27. Braendle, C., Heyland, A., and Flatt, T. (2011). Integrating mechanistic and evolutionary analysis of life history variation. In Flatt, T. and Heyland, A., editors, Mechanisms of life history evolution, pages 3-10. Oxford University Press, New York.

28. Brassard, P., Rau, M. E., and Curtis, M. A. (1982). Parasite-induced susceptibility to predation in diplostomiasis. Parasitology, 85(3):495-501.

29. Brooks, M. E., Kristensen, K., van Benthem, K. J., Magnusson, A., Berg, C. W., Nielsen, A., Skaug, H. J., Mächler, M., and Bolker, B. M. (2017). glmmTMB balances speed and flexibility among packages for zero-inflated generalized linear mixed modeling. The $R$ Journal, 9(2):378400 .

30. Brown, C. and Braithwaite, V. A. (2004). Size matters: a test of boldness in eight populations of the poeciliid Brachyraphis episcopi. Animal Behaviour, 68(6):1325-1329.

31. Brown, C., Jones, F., and Braithwaite, V. (2005). In situ examination of boldness-shyness traits in the tropical poeciliid, Brachyraphis episcopi. Animal Behaviour, 70(5):1003-1009.

32. Brown, C. R. and Bomberger Brown, M. (1986). Ectoparasitism as a cost of coloniality in cliff swallows (Hirundo pyrrhonota). Ecology, 67(5):1206-1218.

33. Burley, N. (1980). Clutch overlap and clutch size: alternative and complementary reproductive tactics. The American Naturalist, 115(2):223-246.

34. Burnham, K. K. and Anderson, D. D. (2002). Model selection and multimodel inference: a 
practical information-theoretic approach. Springer-Verlag, New York, 2 edition.

35. Bush, A. O., Fernández, J. C., Esch, G. W., and Seed, J. R. (2001). Parasitisms: the diversity and ecology of animal parasites. Cambridge University Press, Cambridge.

36. Bussing, W. A. (2002). Peces de las aguas continentales de Costa Rica. Revista de Biologia Tropical, San Jose, 2 edition.

37. Calder, W. A. (1984). Size, function, and life history. Harvard University Press, London.

38. Chambers, R. C., Leggett, W. C., and Brown, J. A. (1989). Egg size, female effects, and the correlations between early life history traits of capelin, Mallotus villosus: an appraisal at the individual level. Fishery Bulletin, 87(3):515-523.

39. Chapman, B. B., Morrell, L. J., and Krause, J. (2010). Unpredictability in food supply during early life influences boldness in fish. Behavioral Ecology, 21(3):501-506.

40. Chick, J. H. and Van Den Avyle, M. J. (2000). Effects of feeding ration on larval swimming speed and responsiveness to predator attacks: implications for cohort survival. Canadian Journal of Fisheries and Aquatic Sciences, 57(1):106-115.

41. Cocheret de la Morinière, E., Pollux, B. J. A., Nagelkerken, I., Hemminga, M. A., Huiskes, A. H. L., and Van der Velde, G. (2003a). Ontogenetic dietary changes of coral reef fishes in the mangrove-seagrass-reef continuum: stable isotopes and gut-content analysis. Marine Ecology Progress Series, 246:279-289.

42. Cocheret de la Morinière, E., Pollux, B. J. A., Nagelkerken, I., and van der Velde, G. (2002). Postsettlement life cycle migration patterns and habitat preference of coral reef fish that use seagrass and mangrove habitats as nurseries. Estuarine, Coastal and Shelf Science, 55(2):309-321.

43. Cocheret de la Morinière, E., Pollux, B. J. A., Nagelkerken, I., and Van Der Velde, G. (2003b). Diet shifts of Caribbean grunts (Haemulidae) and snappers (Lutjanidae) and the relation with nursery-to-coral reef migrations. Estuarine, Coastal and Shelf Science, 57(5-6):1079-1089.

44. Coltman, D. W., Pilkington, J. G., Smith, J. A., and Pemberton, J. M. (1999). Parasite-mediated selection against inbred Soay sheep in a free-living, island population. Evolution, 53(4):1259-1267.

45. Congdon, J. D. and Gibbons, J. W. (1987). Morphological constraint on egg size: a challenge to optimal egg size theory? Proceedings of the National Academy of Sciences of the United States of America, 84(12):4145-4147.

46. Connor, K. L., Kibschull, M., Matysiak-Zablocki, E., Tu-Thu Ngoc Nguyen, T., Matthews, S. G., Lye, S. J., and Bloise, E. (2020). Maternal malnutrition impacts placental morphology and transporter expression: an origin for poor offspring growth. Journal of Nutritional Biochemistry, 78:1-13.

47. Copp, G. H. and Jurajda, P. (1993). Do small riverine fish move inshore at night? Journal of Fish Biology, 43:229-241.

48. Crespi, B. J. and Semeniuk, C. (2004). Parent-offspring conflict in the evolution of vertebrate reproductive mode. The American Naturalist, 163(5):635-653.

49. Cureton, J. C., Martin, R. E., Lewis, R. L., Stoops, S. B., and Deaton, R. (2011). Effects of a trematode infestation on body condition, reproduction and mating behaviors in a livebearing fish. Behaviour, 148(8):967-984.

50. Darwin, C. (1859). On the origin of the species by natural selection.

51. Decaestecker, E., Declerck, S., De Meester, L., and Ebert, D. (2005). Ecological implications of parasites in natural Daphnia populations. Oecologia, 144(3):382-390. 
52. Dekker, M. L., Hagmayer, A., Leon-Kloosterziel, K. M., Furness, A. I., and Pollux, B. J. A. (2020). High degree of multiple paternity and reproductive skew in the highly fecund live-bearing fish Poecilia gillii (Family Poeciliidae). Frontiers in Ecology and Evolution, 8:1-14.

53. Descamps, S., Boutin, S., Berteaux, D., and Gaillard, J.-M. (2008). Age-specific variation in survival, reproductive success and offspring quality in red squirrels. Oikos, 117(9):1406-1416.

54. Dial, T. R., Reznick, D. N., Brainerd, E. L., and Marshall, D. (2016). Effects of neonatal size on maturity and escape performance in the Trinidadian guppy. Functional Ecology, 30(6):943-952.

55. Downhower, J. F. and Brown, L. (1975). Superfoetation in fishes and the cost of reproduction. Nature, 256:345-345.

56. Downhower, J. F., Brown, L. P., and Matsui, M. L. (2000). Life history variation in female Gambusia hubbsi. Environmental Biology of Fishes, 59(4):415-428.

57. Elliot, M. G. and Crespi, B. J. (2006). Placental invasiveness mediates the evolution of hybrid inviability in mammals. The American Naturalist, 168(1):114-120.

58. Elphick, M. C., Hull, D., and Broughton Pipkin, F. (1979). The transfer of fatty acids across the sheep placenta. Journal of Developmental Physiology, 1(1):31-45.

59. Evans, J. P., Pilastro, A., and Schlupp, I. (2011). Ecology and evolution of poeciliid fishes. University of Chicago Press, Chicago.

60. Falconer, D. S. and Mackay, T. F. C. (1996). Introduction to Quantitative Genetics. Pearson Longman, Harlow, 4 edition.

61. Festa-Bianchet, M. (1989). Individual differences, parasites, and the costs of reproduction for bighorn ewes (Ovis canadensis). Journal of Animal Ecology, 58(3):785-795.

62. Fick, S. E. and Hijmans, R. J. (2017). WorldClim 2: new 1-km spatial resolution climate surfaces for global land areas. International Journal of Climatology, 37(12):4302-4315.

63. Filla, M., Premier, J., Magg, N., Dupke, C., Khorozyan, I., Waltert, M., Bufka, L., and Heurich, M. (2017). Habitat selection by Eurasian lynx (Lynx lynx) is primarily driven by avoidance of human activity during day and prey availability during night. Ecology and Evolution, 7(16):63676381.

64. Fleuren, M., Quicazan-Rubio, E. M., van Leeuwen, J. L., and Pollux, B. J. A. (2018). Why do placentas evolve? Evidence for a morphological advantage during pregnancy in live-bearing fish. PLOS ONE, 13(4):1-15.

65. Fleuren, M., van Leeuwen, J. L., and Pollux, B. J. A. (2019). Superfetation reduces the negative effects of pregnancy on the fast-start escape performance in live-bearing fish. Proceedings of the Royal Society B, 286:1-9.

66. Fox, C. W. and Czesak, M. E. (2000). Evolutionary ecology of progeny size in arthropods. Annual Review of Entomology, 45:341-369.

67. Fraser, D. F., Gilliam, J. F., Akkara, J. T., Albanese, B. W., and Sunny, B. S. (2004). Night feeding by guppies under predator release: effects on growth and daytime courtship. Ecology, 85(2):312-319.

68. Fraser, N. H., Metcalfe, N. B., and Thorpe, J. E. (1993). Temperature-dependent switch between diurnal and nocturnal foraging in salmon. Proceedings of the Royal Society B: Biological Sciences, 252(1334):135-139.

69. Freckleton, R. P. (2011). Dealing with collinearity in behavioural and ecological data: model averaging and the problems of measurement error. Behavioral Ecology and Sociobiology, 65(1):91101. 
70. Frith, H. R. (1990). Energetics of fast-starts in northern pike, Esox lucius. PhD thesis, The University of British Columbia.

71. Fry, H., Fry, K., and Harris, A. (1999). Kingfishers, bee-eaters and rollers: an identification guide. Gardners Books.

72. Fuiman, L. A. and Higgs, D. M. (1997). Ontogeny, growth and the recruitment process. In Chambers, R. C. and Trippel, E. A., editors, Early life history and recruitment in fish populations, pages 225-250. Chapman \& Hall, London.

73. Furness, A. I., Avise, J. C., Pollux, B. J. A., Reynoso, Y., and Reznick, D. N. (sub.). The evolution of the placenta in poeciliid fishes: testing adaptive hypotheses.

74. Furness, A. I., Hagmayer, A., and Pollux, B. J. A. (2020). Size-dependent male mating tactics and their morphological correlates in Poecilia gillii. Biological Journal of the Linnean Society, pages $1-18$.

75. Furness, A. I., Pollux, B. J. A., Meredith, R. W., Springer, M. S., and Reznick, D. N. (2019). How conflict shapes evolution in poeciliid fishes. Nature Communications, 10(3335):1-12.

76. Gadgil, M. and Bossert, W. H. (1970). Life historical consequences of natural selection. The American Naturalist, 104(935):1-24.

77. Garrigues, R. and Dean, R. (2014). The birds of costa rica: a field guide. Cornell University Press, Ithaca, New York.

78. Ghalambor, C. K., Reznick, D. N., and Walker, J. A. (2004). Constraints on adaptive evolution: the functional trade-off between reproduction and fast-start swimming performance in the Trinidadian guppy (Poecilia reticulata). The American Naturalist, 164(1):38-50.

79. Gibb, A. C., Swanson, B. O., Wesp, H., Landels, C., and Liu, C. (2006). Development of the escape response in teleost fishes: do ontogenetic changes enable improved performance? Physiological and Biochemical Zoology: Ecological and Evolutionary Approaches, 79(1):7-19.

80. Gibney, E. H., Kipar, A., Rosbottom, A., Guy, C. S., Smith, R. F., Hetzel, U., Trees, A. J., and Williams, D. J. (2008). The extent of parasite-associated necrosis in the placenta and foetal tissues of cattle following Neospora caninum infection in early and late gestation correlates with foetal death. International Journal for Parasitology, 38(5):579-588.

81. Giles, N. (1983). Behavioural effects of the parasite Schistocephalus solidus (Cestoda) on an intermediate host, the three-spined stickleback, Gasterosteus aculeatus L. Animal Behaviour, 31(4):1192-1194.

82. Gliwicz, Z. M. and Guisande, C. (1992). Family planning in Daphnia: resistance to starvation in offspring born to mothers grown at different food levels. Oecologia, 91(4):463-467.

83. Godin, J.-G. J. and Smith, S. A. (1988). A fitness cost of foraging in the guppy. Nature, 333(6168):69-71.

84. Gorini-Pacheco, B., Zandonà, E., and Mazzoni, R. (2017). Predation effects on matrotrophy, superfetation and other life history traits in Phalloceros harpagos. Ecology of Freshwater Fish, 27:442-452.

85. Grueber, C. E., Nakagawa, S., Laws, R. J., and Jamieson, I. G. (2011). Multimodel inference in ecology and evolution: challenges and solutions. Journal of Evolutionary Biology, 24(4):699-711.

86. Gulland, F. (1995). Impact of infectious diseases on wild animal populations: a review. In Grenfell, B. T. and Dobson, A. P., editors, Ecology of Infectious Diseases in Natural Populations, pages 20-51. Cambridge University Press, Cambridge.

87. Hadfield, J. D. (2010). MCMC methods for multi-response generalized linear mixed models: the 
MCMCglmm R package. Journal of Statistical Software, 33(2):1-22.

88. Hagmayer, A., Camenisch, G., Canale, C., Postma, E., and Bonnet, T. (2020a). Limited massindependent individual variation in resting metabolic rate in a wild population of snow voles (Chionomys nivalis). Journal of Evolutionary Biology, 33(5):608-618.

89. Hagmayer, A., Furness, A. I., and Pollux, B. J. A. (2020b). Parasite infestation influences life history but not boldness behavior in placental live-bearing fish. Oecologia, 194:635-648.

90. Hagmayer, A., Furness, A. I., Reznick, D. N., Dekker, M. L., and Pollux, B. J. A. (2020c). Predation risk shapes the degree of placentation in natural populations of live-bearing fish. Ecology Letters, 23(5):831-840.

91. Hagmayer, A., Furness, A. I., Reznick, D. N., and Pollux, B. J. A. (2018). Maternal size and body condition predict the amount of post-fertilization maternal provisioning in matrotrophic fish. Ecology and Evolution, 8(24):12386-12396.

92. Haig, D. (1993). Genetic conflicts in human pregnancy. The Quarterly Review of Biology, $68(4): 495-532$.

93. Hamlett, W. C., Eulitt, A. M., Jarrell, R. L., and Kelly, M. A. (1993). Uterogestation and placentation in Elasmobranchs. The Journal of Experimental Zoology, 266:347-367.

94. Hansen, T. F. and Martins, E. P. (1996). Translating between microevolutionary process and macroevolutionary patterns: the correlation structure of interspecific data. Evolution, 50(4):14041417.

95. Hauer, R. F. and Lamberti, G. A. (2007). Methods in stream ecology. Elsevier, Amsterdam, 2 edition.

96. Haynes, J. L. (1995). Standardized classification of Poeciliid development for life-history studies. Copeia, 1995(1):147-154.

97. Helfman, G. S. (1986). Fish behaviour by day, night and twilight. In Pitcher, T. J., editor, Behaviour of Teleost Fishes, pages 366-387. Springer-Verlag, Boston.

98. Hoffman, G. L. and Putz, R. E. (1965). The black-spot (Uvulifer ambloplitis: Trematoda: Strigeoidea) of Centrarchid fishes. Transactions of the American Fisheries Society, 94(2):143-151.

99. Hunter, G. W. and Hunter, W. S. (1938). Studies on host reactions to larval parasites. I. The effect on weight. The Journal of Parasitology, 24(6):477-481.

100. Hurd, H. (2001). Host fecundity reduction: a strategy for damage limitation? Trends in Parasitology, 17(8):363-368.

101. Jennions, M. D., Wong, B. B. M., Cowling, A., and Donnelly, C. (2006). Life-history phenotypes in a live-bearing fish Brachyrhaphis episcopi living under different predator regimes: seasonal effects? Environmental Biology of Fishes, 76:211-219.

102. Johnson, J. B. and Belk, M. C. (2001). Predation environment predicts divergent life-history phenotypes among populations of the livebearing fish Brachyrhaphis rhabdophora. Oecologia, 126(1):142-149.

103. Jørgensen, C., Auer, S. K., and Reznick, D. N. (2011). A model for optimal offspring size in fish, including live-bearing and parental effects. The American Naturalist, 177(5):E119-E135.

104. Kaartvedt, S., Klevjer, T. A., Torgersen, T., Sørnes, T. A., and Røstad, A. (2007). Diel vertical migration of individual jellyfish (Periphylla periphylla). Limnology and Oceanography, 52(3):975983.

105. Kaplan, R. H. (1992). Greater maternal investment can decrease offspring survival in the frog 
Bombina orientalis. Ecology, 73(1):280-288.

106. Kern, S., Ackermann, M., Stearns, S. C., and Kawecki, T. J. (2001). Decline in offspring viability as a manifestation of aging in Drosophila melanogaster. Evolution, 55(9):1822-1831.

107. Koehler, A. V., Brown, B., Poulin, R., Thieltges, D. W., and Fredensborg, B. L. (2012). Disentangling phylogenetic constraints from selective forces in the evolution of trematode transmission stages. Evolutionary Ecology, 26(6):1497-1512.

108. Kortet, R., Hedrick, A. V., and Vainikka, A. (2010). Parasitism, predation and the evolution of animal personalities. Ecology Letters, 13(12):1449-1458.

109. Kramer, D. L., Manley, D., and Bourgeois, R. (1983). The effect of respiratory mode and oxygen concentration on the risk of aerial predation in fishes. Canadian Journal of Zoology, 61(3):653665 .

110. Krause, J., Loader, S. P., McDermott, J., and Ruxton, G. D. (1998). Refuge use by fish as a function of body length-related metabolic expenditure and predation risks. Proceedings of the Royal Society B: Biological Sciences, 265(1413):2373-2379.

111. Kuznetsova, A., Brockhoff, P. B., and Christensen, R. H. B. (2016). lmerTest: tests for random and fixed effects for linear mixed effect models.

112. Kwan, L., Fris, M., Rodd, F. H., Rowe, L., Tuhela, L., and Panhuis, T. M. (2015). An examination of the variation in maternal placentae across the genus Poeciliopsis (Poeciliidae). Journal of Morphology, 276(6):707-720.

113. Laidlaw, C. T., Condon, J. M., and Belk, M. C. (2014). Viability costs of reproduction and behavioral compensation in western mosquitofish (Gambusia affinis). PLOS ONE, 9(11):1-5.

114. Lande, R. (1976). Natural selection and random genetic drift in phenotypic evolution. Evolution, 30:314-334.

115. Lande, R. and Arnold, S. J. (1983). The measurement of selection on correlated characters. Evolution, 37(6):1210-1226.

116. Lane, R. L. and Morris, J. E. (2010). Biology, prevention, and effects of common grubs (digenetic trematodes) in freshwater fish. NCRAC Technical Bulletins, 14:1-6.

117. Lankheet, M. J., Stoffers, T., van Leeuwen, J. L., and Pollux, B. J. A. (2016). Acquired versus innate prey capturing skills in super-precocial live-bearing fish. Proceedings of the Royal Society B: Biological Sciences, 283(1834):1-9.

118. Lanza, J. (1983). Microhabitat use by bisexual and unisexual fishes (Poeciliopsis: Poeciliidae) in an artificial stream. Oecologia, 57:142-147.

119. Law, R. (1979). Optimal life histories under age-specific predation. The American Naturalist, 114(3):399-417.

120. Leips, J., Rodd, F. H., and Travis, J. (2013). The adaptive significance of population differentiation in offspring size of the least killifish, Heterandria formosa. Ecology and Evolution, $3(4): 948-960$.

121. Lemly, A. D. . and Esch, G. W. (1984). Effects of the trematode Uvulifer ambloplitis on juvenile bluegill sunfish. Journal of Parasitology, 70(4):475-492.

122. Lively, C. M., Craddock, C., and Vrijenhoek, R. C. (1990). Red Queen hypothesis supported by parasitism in sexual and clonal fish. Nature, 344:864-866.

123. Losos, J. B., Creer, D. A., and Schulte, J. A. (2002). Cautionary comments on the measurement of maximum locomotor capabilities. Journal of Zoology, 258(1):57-61. 
124. Losos, J. B., Jackman, T. R., Larson, A., de Queiroz, K., and Rodriguez-Schettino, L. (1998). Contingency and determinism in replicated adaptive radiations of island lizards. Science, 279:2115-2118.

125. Lowe-McConnell, R. H. (1975). Fish communities in tropical freshwaters: their distribution, ecology, and evolution. Longman, New York.

126. MacArthur, R. H. and Pianka, E. R. (1966). On optimal use of a patchy environment. The American Naturalist, 100(916):603-609.

127. Marcogliese, D. J. (2016). The distribution and abundance of parasites in aquatic ecosystems in a changing climate: more than just temperature. Integrative and Comparative Biology, 56(4):611619 .

128. Marsh-Matthews, E. (2011). Matrotrophy. In Evans, J. P., Pilastro, A., and Schlupp, I., editors, Ecology and Evolution of Poeciliid Fishes, chapter 2, pages 18-27. University of Chicago Press, Chicago.

129. Marshall, D. J., Heppell, S. S., Munch, S. B., and Warner, R. R. (2010). The relationship between maternal phenotype and offspring quality: do older mothers really produce the best offspring? Ecology, 91(10):2862-2873.

130. Marshall, D. J. and Keough, M. J. (2008a). The evolutionary ecology of offspring size in marine invertebrates. Advances in Marine Biology, 53:1-60.

131. Marshall, D. J. and Keough, M. J. (2008b). The relationship between offspring size and performance in the sea. The American Naturalist, 171(2):214-224.

132. Matheney IV, M. P. and Rabeni, C. F. (1995). Patterns of movement and habitat use by northern hog suckers in an ozark stream. Transactions of the American Fisheries Society, 124:886-897.

133. McIntyre, G. S. and Gooding, R. H. (2000). Egg size, contents, and quality: maternal-age and -size effects on house fly eggs. Canadian Journal of Zoology, 78(9):1544-1551.

134. Menard, S. (2011). Standards for standardized logistic regression coefficients. Social Forces, 89(4):1409-1428.

135. Metcalfe, N. B., Fraser, N. H., and Burns, M. D. (1999). Food availability and the nocturnal vs. diurnal foraging trade-off in juvenile salmon. Journal of Animal Ecology, 68(2):371-381.

136. Metcalfe, N. B. and Monaghan, P. (2001). Compensation for a bad start: grow now, pay later? Trends in Ecology 83 Evolution, 16(5):254-260.

137. Meyer, M. K. (2015). Lebendgebärende Zierfische. Chimaira, band 1 edition.

138. Michalakis, Y. and Hochberg, M. E. (1994). Parasitic effects on host life-history traits: a review of recent studies. Parasite, 1(4):291-294.

139. Michod, R. E. (1979). Evolution of life histories in response to age-specific mortality factors. The American Naturalist, 113(4):531-550.

140. Mittelbach, G. G. (1981). Foraging efficiency and body size: a study of optimal diet and habitat use by bluegills. Ecology, 62(5):1370-1386.

141. Møller, A. P. (1990). Effects of parasitism by a haematophagous mite on reproduction in the barn swallow. Ecology, 71(6):2345-2357.

142. Morris, D. W. (2003). Toward an ecological synthesis: a case for habitat selection. Oecologia, 136(1):1-13.

143. Mossman, H. W. (1987). Vertebrate fetal membranes. Rutgers University Press, New Brunswick.

144. Mousseau, T. A. and Fox, C. W. (1998a). Maternal effects as adaptations. Oxford University 
Press, Oxford.

145. Mousseau, T. A. and Fox, C. W. (1998b). The adaptive significance of maternal effects. Trends in Ecology 8 Evolution, 13(10):403-407.

146. Noren, S. R., Redfern, J. V., and Edwards, E. F. (2011). Pregnancy is a drag: hydrodynamics, kinematics and performance in pre- and post-parturition bottlenose dolphins (Tursiops truncatus). Journal of Experimental Biology, 214(24):4151-4159.

147. Olivera-Tlahuel, C., Moreno-Mendoza, N. A., Villagrán-Santa Cruz, M., and Zúñiga-Vega, J. J. (2019). Placental structures and their association with matrotrophy and superfetation in poeciliid fishes. Acta Zoologica, 100:167-181.

148. Ostrovsky, A. N., Lidgard, S., Gordon, D. P., Schwaha, T., Genikhovich, G., and Ereskovsky, A. V. (2016). Matrotrophy and placentation in invertebrates: a new paradigm. Biological Reviews of the Cambridge Philosophical Society, 91(3):673-711.

149. Parker, G. A. and Begon, M. (1986). Optimal egg size and clutch size: effects of environment and maternal phenotype. The American Naturalist, 128(4):573-592.

150. Penaluna, B. E., Dunham, J. B., and Noakes, D. L. (2016). Instream cover and shade mediate avian predation on trout in semi-natural streams. Ecology of Freshwater Fish, 25(3):405-411.

151. Petterson, J. A., Slepetis, R., Ehrhardt, R. A., Dunshea, F. R., and Bell, A. W. (1994). Pregnancy but not moderate undernutrition attenuates insulin suppression of fat mobilization in sheep. Journal of Nutrition, 124(12):2431-2436.

152. Pianka, E. R. and Parker, W. S. (1975). Age-specific reproductive tactics. The American Naturalist, 109(968):453-464.

153. Pichler, C. and Schiemer, F. (2008). Ecology of fishes of Quebrada Negra, Costa Rica, a first order neotropical lowland stream. Stapfia, 88:495-505.

154. Piet, G. and Guruge, W. A. H. P. (1997). Diel variation in feeding and vertical distribution of ten co-occurring fish species: consequences for resource partitioning. Environmental Biology of Fishes, 50:293-307.

155. Pires, M. N., Arendt, J., and Reznick, D. N. (2010). The evolution of placentas and superfetation in the fish genus Poecilia (Cyprinodontiformes: Poeciliidae: Subgenera Micropoecilia and Acanthophacelus). Biological Journal of the Linnean Society, 99(4):784-796.

156. Pires, M. N., Bassar, R. D., McBride, K. E., Regus, J. U., Garland, T., and Reznick, D. N. (2011). Why do placentas evolve? An evaluation of the life-history facilitation hypothesis in the fish genus Poeciliopsis. Functional Ecology, 25(4):757-768.

157. Pires, M. N., McBride, K. E., and Reznick, D. N. (2007). Interpopulation variation in lifehistory traits of Poeciliopsis prolifica: implications for the study of placental evolution. Journal of Experimental Zoology, 307:113-125.

158. Plath, M., Riesch, R., Culumber, Z., Streit, B., and Tobler, M. (2011). Giant water bug (Belostoma sp.) predation on a cave fish (Poecilia mexicana): effects of female body size and gestational state. Evolutionary Ecology Research, 13(2):133-144.

159. Plaut, I. (2002). Does pregnancy affect swimming performance of female Mosquitofish, Gambusia affinis? Functional Ecology, 16:290-295.

160. Plummer, M. (2003). JAGS: a program for analysis of Bayesian graphical models using Gibbs sampling.

161. Pollux, B. J. A., Meredith, R. W., Springer, M. S., Garland, T., and Reznick, D. N. (2014). The evolution of the placenta drives a shift in sexual selection in livebearing fish. Nature, 
513(7517):233-236.

162. Pollux, B. J. A., Pires, M. N., Banet, A. I., and Reznick, D. N. (2009). Evolution of placentas in the fish family Poeciliidae: an empirical study of macroevolution. Annual Review of Ecology, Evolution, and Systematics, 40:271-289.

163. Pollux, B. J. A. and Reznick, D. N. (2011). Matrotrophy limits a female's ability to adaptively adjust offspring size and fecundity in fluctuating environments. Functional Ecology, 25:747-756.

164. Pollux, B. J. A., Verberk, W. C. E. P., Dorenbosch, M., Cocheret de la Morinière, E., Nagelkerken, I., and Van der Velde, G. (2007). Habitat selection during settlement of three Caribbean coral reef fishes: indications fordirected settlement to seagrass beds and mangroves. Limnology and Oceanography, 52(2):903-907.

165. Poulin, R. (2006). Global warming and temperature-mediated increases in cercarial emergence in trematode parasites. Parasitology, 132(1):143-151.

166. Power, M. E. (1984). Depth distributions of armored catfish: predator-induced resource avoidance? Ecology, 65(2):523-528.

167. Prentice, A. M. and Prentice, A. (1988). Energy costs of lactation. Annual Review of Nutrition, 8(1):63-79.

168. Quicazan-Rubio, E. M., van Leeuwen, J. L., van Manen, K., Fleuren, M., Pollux, B. J. A., and Stamhuis, E. J. (2019). Coasting in live-bearing fish: the drag penalty of being pregnant. Journal of the Royal Society Interface, 16:1-10.

169. R Core Team (2020). R: a language and environment for statistical computing.

170. Reznick, D. N. (1982a). Genetic determination of offspring size in the guppy (Poecilia reticulata). The American Naturalist, 120(2):181-188.

171. Reznick, D. N. (1982b). The impact of predation on life history evolution in Trinidadian guppies: genetic basis of observed life history patterns. Evolution, 36(6):1236-1250.

172. Reznick, D. N. (1983). The structure of guppy life histories: the tradeoff between growth and reproduction. Ecology, 64(4):862-873.

173. Reznick, D. N., Bryant, M. J., Roff, D., Ghalambor, C. K., and Chalambor, D. E. (2004). Effects of extrinsic mortality on the evolution of senescence in guppies. Nature, 431:1095-1099.

174. Reznick, D. N., Bryga, H., and Endler, J. A. (1990). Experimentally induced life-history evolution in a natural population. Nature, 346(6282):357-359.

175. Reznick, D. N. and Bryga, H. A. (1996). Life-history evolution in guppies (Poecilia reticulata: Poeciliidae). V. Genetic basis of parallelism in life histories. The American Naturalist, 147(3):339359.

176. Reznick, D. N., Butler IV, M. J., Rodd, F. H., and Ross, P. (1996a). Life-history evolution in guppies (Poecilia reticulata) VI. Differential mortality as a mechanism for natural selection. Evolution, 50(4):1651-1660.

177. Reznick, D. N., Callahan, H., and Llauredo, R. (1996b). Maternal effects on offspring quality in Poeciliid fishes. American Zoologist, 36(2):147-156.

178. Reznick, D. N. and Endler, J. A. (1982). The impact of predation on life history evolution in Trinidadian guppies (Poecilia reticulata). Evolution, 36(1):160-177.

179. Reznick, D. N., Mateos, M., and Springer, M. S. (2002). Independent origins and rapid evolution of the placenta in the fish genus Poeciliopsis. Science, 298(5595):1018-1020.

180. Reznick, D. N., Meredith, R., and Collette, B. B. (2007). Independent evolution of complex life 
history adaptations in two families of fishes, live-bearing halfbeaks (Zenarchopteridae, Beloniformes) and Poeciliidae (Cyprinodontiformes). Evolution, 61(11):2570-2583.

181. Reznick, D. N. and Miles, D. B. (1989). A review of life history patterns in poeciliid fishes. In Meffe, G. K. and Snelson, F. F., editors, Ecology 83 Evolution of Livebearing Fishes (Poeciliidae), pages 125-148. PrenticeHall, New Jersey.

182. Reznick, D. N. and Ricklefs, R. E. (2009). Darwin's bridge between microevolution and macroevolution. Nature, 457(7231):837-842.

183. Richner, H. (1998). Host-parasite interactions and life-history evolution. Zoology, 101(4):333-344.

184. Robertson, G. N., Lindsey, B. W., Dumbarton, T. C., Croll, R. P., and Smith, F. M. (2008). The contribution of the swimbladder to buoyancy in the adult zebrafish (Danio rerio): a morphometric analysis. Journal of Morphology, 269(6):666-673.

185. Rodewald, A. D. and Foster, S. A. (1998). Effects of gravidity on habitat use and antipredator behaviour in three-spined sticklebacks. Journal of Fish Biology, 52(5):973-984.

186. Roff, D. (1992). Evolution of life histories. Springer US.

187. Rolland, J., Silvestro, D., Litsios, G., Faye, L., and Salamin, N. (2018). Clownfishes evolution below and above the species level. Proceedings of the Royal Society B: Biological Sciences, 285(1873):1-9.

188. Rome, L. C., Funke, R. P., McNeill Alexander, R., Lutz, G., Aldridge, H., Scott, F., and Freadman, M. (1988). Why animals have different muscle fibre types. Nature, 335:824-827.

189. Rosenzweig, M. L. (1981). A theory of habitat selection. Ecology, 62(2):327-335.

190. Rosenzweig, M. L. (1991). Habitat selection and population interactions: the search for mechanism. The American Naturalist, 137:S5-S28.

191. Sainmont, J., Thygesen, U. H., and Visser, A. W. (2013). Diel vertical migration arising in a habitat selection game. Theoretical Ecology, 6(2):241-251.

192. Schielzeth, H. (2010). Simple means to improve the interpretability of regression coefficients. Methods in Ecology and Evolution, 1(2):103-113.

193. Schindler, A. D. W. (1977). Evolution of phosphorus limitation in lakes. Science, 195(4275):260262.

194. Schlosser, I. J. (1988). Predation risk and habitat selection by two size classes of a stream cyprinid: experimental test of a hypothesis. Oikos, 52(1):36-40.

195. Schrader, M. and Travis, J. (2005). Population differences in pre- and post-fertilization offspring provisioning in the least killifish, Heterandria formosa. Copeia, 2005(3):649-656.

196. Seigel, R. A., Huggins, M. M., and Ford, N. B. (1987). Reduction in locomotor ability as a cost of reproduction in gravid snakes. Oecologia, 73(4):481-485.

197. Sempeski, P. and Gaudin, P. (1995). Size-related changes in diel distribution of young grayling (Thymallus thymallus). Canadian Journal of Fisheries and Aquatic Sciences, 52:1842-1848.

198. Sheldon, B. C. and Verhulst, S. (1996). Ecological immunology: costly parasite defences and trade-offs in evolutionary ecology. Trends in Ecology \& Evolution, 11(8):317-321.

199. Shine, R. (1992). Relative clutch mass and body shape in lizards and snakes: is reproductive investment constrained or optimized? Evolution, 46(3):828-833.

200. Sibly, R. and McFarland, D. (1976). On the fitness of behavior sequences. The American Naturalist, 110(974):601-617. 
201. Sibly, R. M. and Calow, P. (1983). An integrated approach to life cycle evolution using selective landscapes. Journal of Theoretical Biology, 102:527-547.

202. Sih, A. (1980). Optimal behavior: can foragers balance two conflicting demands? Science, 210(4473):1041-1043.

203. Sih, A., Bell, A. M., Chadwick Johnson, J., and Ziemba, R. E. (2004). Behavioral syndromes: an integrative overview. Quarterly Review of Biology, 79(3):241-277.

204. Skalski, G. T. and Gilliam, J. F. (2002). Feeding under predation hazard: testing models of adaptive behavior with stream fish. The American Naturalist, 160(2):158-172.

205. Sogard, S. M. and Olla, B. L. (2002). Contrasts in the capacity and underlying mechanisms for compensatory growth in two pelagic marine fishes. Marine Ecology Progress Series, 243:165-177.

206. Stearns, S. C. (1992). The evolution of life histories, volume 249. Oxford University Press, Oxford.

207. Swing, C. K. (1992). Influences on the instream distribution of Astyanax fasciatus in a small river in Costa Rica. PhD thesis.

208. Tessier, A. J., Henry, L. L., Goulden, C. E., and Durand, M. W. (1983). Starvation in Daphnia: energy reserves and reprodutive allocation. Limnololgy and Oceanography, 28(4):667-676.

209. Thibault, R. E. and Schultz, R. J. (1978). Reproductive adaptations among viviparous fishes (Cyprinodontiformes: Poeciliidae). Evolution, 32(2):320-333.

210. Tobler, M., Plath, M., Burmeister, H., and Schlupp, I. (2006). Black spots and female association preferences in a sexual/asexual mating complex (Poecilia, Poeciliidae, Teleostei). Behavioral Ecology and Sociobiology, 60(2):159-165.

211. Tobler, M. and Schlupp, I. (2008). Influence of black spot disease on shoaling behaviour in female western mosquitofish, Gambusia affinis (Poeciliidae, Teleostei). Environmental Biology of Fishes, $81(1): 29-34$.

212. Tobler, M., Schlupp, I., García de León, F. J., Glaubrecht, M., and Plath, M. (2007). Extreme habitats as refuge from parasite infections? Evidence from an extremophile fish. Acta Oecologica, 31(3):270-275.

213. Tobler, M., Wahli, T., and Schlupp, I. (2005). Comparison of parasite communities in native and introduced populations of sexual and asexual mollies of the genus Poecilia. Journal of Fish Biology, 67(4):1072-1082.

214. Torrico, F., Alonso-Vega, C., Suarez, E., Rodriguez, P., Torrico, M. C., Dramaix, M., Truyens, C., and Carlier, Y. (2004). Maternal Trypanosoma cruzi infection, pregnancy outcome, morbidity, and mortality of congenitally infected and non-infected newborns in Bolivia. American Journal of Tropical Medicine and Hygiene, 70(2):201-209.

215. Travis, J., Farr, J. A., Henrich, S., and Cheong, R. T. (1987). Testing theories of clutch overlap with the reproductive ecology of Heterandria formosa. Ecology, 68(3):611-623.

216. Trexler, J. C. (1997). Resource availability and plasticity in offspring provisioning: embryo nourishment in sailfin mollies. Ecology, 78(5):1370-1381.

217. Trexler, J. C. and DeAngelis, D. L. (2003). Resource allocation in offspring provisioning: an evaluation of the conditions favoring the evolution of matrotrophy. The American Naturalist, $162(5): 574-585$.

218. Trexler, J. C. and DeAngelis, D. L. (2010). Modeling the evolution of complex reproductive adaptations in Poeciliid fishes: matrotrophy and superfetation. In Uribe, M. C. and Grier, H. J., editors, Viviparous fishes II, pages 231-240. New Life Publications. 
219. Trexler, J. C., Tempe, R. C., and Travis, J. (1994). Size-selective predation of sailfin mollies by two species of heron. Oikos, 69(2):250-258.

220. Trivers, R. L. (1974). Parent-offspring conflict. American Zoologist, 14:249-264.

221. Turcotte, M. M., Pires, M. N., Vrijenhoek, R. C., and Reznick, D. N. (2008). Pre- and postfertilization maternal provisioning in livebearing fish species and their hybrids (Poeciliidae: Poeciliopsis). Functional Ecology, 22(6):1118-1124.

222. Turner, C. L. (1937). Reproductive cycles and superfetation in poeciliid fishes. Biological Bulletin, $72(2): 145-164$.

223. Turner, C. L. (1940). Pseudoamnion, pseudochorion, and follicular pseudoplacenta in poeciliid fishes. Journal of Morphology, 67(1):59-89.

224. van der Vinne, V., Tachinardi, P., Riede, S. J., Akkerman, J., Scheepe, J., Daan, S., and Hut, R. A. (2019). Maximising survival by shifting the daily timing of activity. Ecology Letters, 22:2097-2102.

225. van Noordwijk, A. J. and de Jong, G. (1986). Acquisition and allocation of resources: their influence on variation in life history tactics. The American Naturalist, 128(1):137-142.

226. Veggetti, A., Mascarello, F., Scapolo, P. A., Rowlerson, A., and Candia Carnevali, M. D. (1993). Muscle growth and myosin isoform transitions during development of a small teleost fish, Poecilia reticulata (Peters) (Atheriniformes, Poeciliidae): a histochemical, immunohistochemical, ultrastructural and morphometric study. Anatomy and Embryology, 187:353-361.

227. Voesenek, C. J., Li, G., Muijres, F. T., and van Leeuwen, J. L. (2020). Experimental-numerical method for calculating bending moments in swimming fish shows that fish larvae control undulatory swimming with simple actuation. PLoS Biology, 18(7):1-24.

228. Wake, M. H. (2015). Fetal adaptations for viviparity in amphibians. Journal of Morphology, 276:941-960.

229. Wakeling, J. M. (2006). Fast-start mechanics. In Shadwick, R. E. and Lauder, G. V., editors, Fish Physiology: fish biomechanics, volume 23, chapter 9, pages 333-368. Academic Press.

230. Walker, J. A., Chalambor, C. K., Griset, O. L., McKenney, D., and Reznick, D. N. (2005). Do faster starts increase the probability of evading predators? Functional Ecology, 19:808-815.

231. Wallace, R. A. and Selman, K. (1981). Cellular and dynamic aspects of oocyte growth in teleosts. American Zoologist, 21:325-343.

232. Werner, E. and Hall, D. (1988). Ontogenetic habitat shifts in bluegill: the foraging rate-predation risk trade-off. Ecology, 69(5):1352-1366.

233. Werner, E. E. (1984). The ontogenetic niche and species interaction. Annual Review of Ecology and Systematics, 15:393-425.

234. Whitfield, A. K. and Cyrus, D. P. (1978). Feeding succession and zonation of aquatic birds at false bay, Lake St Lucia. Ostrich, 49(1):8-15.

235. Wooding, P. and Burton, G. (2008). Comparative placentation: structures, functions, and evolution. Springer Verlag, Berlin.

236. Wourms, J. P. (1981). Viviparity: the maternal-fetal relationship in fishes. American Zoologist, 21(2):473-515.

237. Zeh, D. W. and Zeh, J. A. (2000). Reproductive mode and speciation: the viviparity-driven conflict hypothesis. Bioessays, 22:938-946.

238. Zera, A. J. and Harshman, L. G. (2001). The physiology of life history trade-offs in animals. 
Annual Review of Ecology and Systematics, 32:95-126.

239. Zúñiga-Vega, J. J., Macías-Garcia, C., and Johnson, J. B. (2010). Hypotheses to explain the evolution of superfetation in vivparous fishes. In Uribe, M. C. and Grier, H. J., editors, Viviparous fishes II, pages 241-253. New Life Publications, Homestead.

240. Zúñiga-Vega, J. J., Reznick, D. N., and Johnson, J. B. (2007). Habitat predicts reproductive superfetation and body shape in the livebearing fish Poeciliopsis turrubarensis. Oikos, 116(6):9951005. 
Summaries 


\section{Summary}

Understanding how ecology influences the evolution of complex life-history traits is of fundamental interest to ecologists and evolutionary biologists alike. The placenta is an excellent example of a complex life-history trait that has evolved repeatedly - and continues to evolve to date - throughout the animal kingdom. In the live-bearing fish family Poeciliidae alone, the placenta evolved at least nine times independently. This repeated evolution and ongoing elaboration appears to point towards an adaptive advantage to specific environmental conditions; however, this potential benefit is currently insufficiently understood. In this thesis, I attempt to shed light on the causes and consequences of placental evolution by drawing on insights from the live-bearing fish family Poeciliidae.

In Chapter 2, we studied maternal causes and consequences of embryo provisioning during gestation in the placental live-bearing fish species Poeciliopsis retropinna from Costa Rica. Specifically, we examined how maternal traits (i.e. body fat, lean mass, and length) relate to pre- (i.e. allocation to the egg prior to fertilization) and post-fertilization (i.e. allocation to the embryo during pregnancy) maternal provisioning and how this ultimately affects offspring size and body composition at birth. We showed that maternal traits strongly correlate with embryo size and body composition throughout pregnancy, though different maternal traits are differently related to pre- and post-fertilization maternal provisioning. From our results, we conclude that (i) embryo size and offspring size at birth are plastic phenotypic traits that are predicted by the maternal phenotype, and (ii) maternal provisioning is not constant throughout pregnancy, but depends on the developmental stage of the embryos.

In Chapter 3, we studied 28 natural populations of the placental live-bearing fish species $P$. retropinna in Costa Rica to test a key prediction of the locomotor cost hypothesis, which is that the placenta evolves in high 'performance-demanding' environments by gradually shifting maternal investment from pre- to post-fertilization, thereby reducing the reproductive burden experienced by females during pregnancy and improving their locomotor performance. We confirmed two key predictions of this hypothesis, which are that (i) placentas evolve in natural populations in response to high predation conditions and (ii) that this significantly reduces a female's reproductive burden during pregnancy. This chapter provides the first evidence for an adaptive (ecological) explanation for why the placenta evolves in natural populations. Moreover, it reveals an unexpected fundamental feature of placental animals, which is that an increase in the degree of placentation can lead to a lower reproductive burden without any apparent reproductive cost: i.e. without sacrificing either fecundity or offspring size and quality at birth.

In Chapter 4, we empirically tested the idea that the evolution of placentation and superfetation facilitates a life in 'performance-demanding' (e.g. high-flow velocity) environments by reducing the reproductive burden of females during pregnancy, and hence, improving 
locomotor performance. If true, we predicted placental species and/or species with superfetation to inhabit higher 'performance-demanding' (micro)habitats compared to closely related non-placental species or species without superfetation. For this, we used underwater visual census to study diurnal and ontogenetic microhabitat selection in Costa Rican rivers by five sympatric live-bearing fish species (family Poeciliidae) that differ in the absence/presence of placentation and superfetation. Consistent with our predictions, we observed significant interspecific differences in daytime microhabitat use: species with placentation and superfetation were found in deeper and faster-flowing parts of the river, species that lacked both adaptations were confined to shallow slow-flowing areas, and species with one adaptation (i.e. only superfetation) inhabited intermediate areas. This interspecific daytime microhabitat use was strongest in reproductive adults, intermediate in immatures, and absent in juveniles, suggesting that ontogeny influences species-specific microhabitat use. Finally, at night, all fishes, regardless of the species or age-class, congregated in shallow slow-flowing waters to rest (sleep) on the river bottom. Our results suggest that placentation and superfetation may be hitherto unrecognized reproductive features that help to understand differences in ontogenetic and diurnal microhabitat preferences between sympatric live-bearing fish species living in environments characterized by large flow variation.

In Chapter 5, we examined the consequences of a trematode infestation (black spot disease, BSD) in shaping life-history and behavior (boldness trials in the field) in the placental livebearing fish species $P$. retropinna from Costa Rica. We proposed that the intimate link the placenta forms between the mother and fetus poses a risk, because parasite infestation may have unfavorable consequences for fetal development. For example, maternal parasite infestation can affect fetal growth in two non-mutually exclusive ways: (i) directly, through infestation of the developing fetus by parasites that can cross the placental barrier; and (ii) indirectly, through the modification of maternal physiology or metabolism to such an extent that it interferes with fetal development. We showed profound variation in parasite infestation among females within populations associated with maternal size and body condition (expressed as maternal fat reserves). Moreover, we found that heavily parasitized females produced smaller and worse-conditioned offspring at birth, possibly because a costly immune response during pregnancy may limit the energy available to (i) nourish developing embryos or (ii) form a well-functioning placenta (indirectly affecting embryo nourishment). However, the infestation rate did not affect an individual's boldness behavior in the field. Our findings show that in placental live-bearing fish parasite infestation leads to reduced embryo provisioning during pregnancy, resulting in a smaller offspring size and quality at birth potentially with negative implications for offspring fitness.

In Chapter 6, we conducted a 7-week laboratory experiment to examine to what extent maternal food limitation during pregnancy affects offspring size and quality (i.e. body fat) at birth, as well as growth and locomotor performance of offspring after birth in 
the placental live-bearing fish species Phalloptychus januarius. We showed that maternal food restriction resulted in a decrease in maternal wet mass throughout the experiment, leading to a reduced resource allocation to $\mathrm{F}_{1}$-offspring size and body fat, which can be compensated only in the long-term (i.e. when adult). Although this did not impact the fast-start escape performance of $\mathrm{F}_{1}$-offspring immediately after birth, it negatively affected the postnatal development of locomotor performance during feeding, and thus, body condition and presumably fitness after birth. The negative effects of maternal food restriction on offspring size still occurred in the $\mathrm{F}_{2}$-generation at birth, but disappeared one week after birth. This chapter suggests that placentotrophy in poeciliids is likely a maladaptive strategy in fluctuating resource environments, because sudden reductions in maternal food availability during pregnancy result in smaller offspring with a lower locomotor performance during early life.

Finally, in Chapter 7, I put the findings of this thesis into a wider scientific context. First, I restate the different hypotheses for the evolution of the placenta together with the currently available evidence for each. Second, because superfetation is commonly found in Poeciliidae and thought to co-evolve with the evolution of the placenta, I developed an R-package to simulate and graphically illustrate the potential advantages of having superfetation, as well as both, superfetation and placentation. The simulation shows that the co-evolution of placentation and superfetation might be favored by the additive effects of both traits on the reproductive burden of females during pregnancy. Third, I discuss the potential disadvantages of having a placenta in response to various adverse environmental conditions. I conclude that although having a placenta offers a selective advantage to females in high 'performance-demanding' environments by improving swimming performance, the intimate link the placenta forms between mother and fetus also poses a risk: maternal exposure to adverse environmental conditions (e.g. malnutrition, parasite infestation) is likely to have unfavorable consequences for fetal development. Finally, I present an outlook on future research by addressing possible steps and challenges to deepen and expand the knowledge on the causes and consequences of placental evolution. 


\section{Zusammenfassung}

Das Verständnis, wie ökologische Faktoren die Evolution von komplexen Merkmalen beeinflusst, ist von fundamentalem Interesse für Ökologen und Evolutionsbiologen. Die Gebärmutter ist ein ideales Beispiel für ein komplexes Merkmal, das im gesamten Tierreich mehrmals unabhängig voneinander evolviert ist und heutzutage immer noch evolviert. Allein in der lebendgebärenden Fischfamilie Poeciliidae ist die Gebärmutter mindestens neun Mal unabhängig voneinander evolviert. Diese wiederholte Evolution und fortlaufende Elaboration der Gebärmutter deutet auf einen adaptiven Vorteil unter bestimmten Umweltbedingungen, jedoch ist dieser noch unzureichend erforscht und verstanden. In dieser Dissertation versuche ich mit Hilfe der Erforschung von lebendgebärenden Fischen der Familie Poeciliidae über die Ursachen und Konsequenzen der Gebärmutter-Evolution Aufschluss zu geben.

In Kapitel 2 studierten wir mütterliche Ursachen und Konsequenzen der embryonalen Nährstoffversorgung während der Schwangerschaft in der lebendgebärenden Fischart Poeciliopsis retropinna von Costa Rica. Im Besonderen untersuchten wir wie mütterliche Merkmale (d.h. Körperfett, Magermasse, und Länge) mit dem Nährstoffinvestment in die Eier vor der Befruchtung und der Nährstoffversorgung der Embryonen nach der Befruchtung während der Schwangerschaft verknüpft sind. Zudem untersuchten wir wie dies schlussendlich die Grösse und Körperkomposition der Nachkommen bei der Geburt beeinflusst. Wir haben gezeigt, dass mütterliche Merkmale mit der Grösse und Körperkomposition der Embryonen während der Schwangerschaft stark korreliert. Jedoch korrelieren die verschiedenen mütterlichen Merkmale unterschiedlich mit der Nährstoffversorgung vor und nach der Befruchtung der Eier. Anhand von unseren Resultaten folgern wir, dass (i) die Grösse von Embryonen und Neugeborenen plastische phänotypische Merkmale, die mit dem mütterlichen Phänotyp korrelieren, sind, und (ii) die mütterliche Nährstoffversorgung während der Schwangerschaft nicht durchgehend konstant ist, sondern vielmehr vom Entwicklungsstadium der Embryonen abhängt.

In Kapitel 3 studierten wir 28 natürliche Populationen der plazental lebendgebärenden Fischart P. retropinna von Costa Rica um eines der Hauptpostulate der 'BeweglichkeitsKosten Hypothese' zu testen. Dieses postuliert, dass die Gebärmutter in einer körperlich anspruchsvollen Umgebung evolviert, nämlich durch die kontinuierliche Verlagerung der mütterlichen Nährstoffversorgung während der Schwangerschaft von den unbefruchteten Eiern zu den Embryonen. Dies reduziert schliesslich die körperliche Belastung während der Schwangerschaft und fördert die Beweglichkeit. Wir bestätigten zwei Hauptpostulate dieser Hypothese: (i) die Gebärmutter evolviert in natürlichen Populationen mit hoher Prädationsgefahr, was folglich (ii) die körperliche Belastung während der Schwangerschaft signifikant reduziert. Dieses Kapitel liefert den ersten Beweis für eine adaptive (ökologische) Erklärung für die Evolution der Gebärmutter in natürlichen Populationen. 
Zudem zeigt es ein unerwartetes Merkmal von Plazentatieren auf, nämlich das eine verstärkte plazentale Nährstoffversorgung der Embryonen zu einer reduzierten körperlichen Belastung während der Schwangerschaft ohne offensichtliche Fortpflanzungskosten führt: Ohne Beeinträchtigung der mütterlichen Fruchtbarkeit oder der Grösse und Qualität der Nachkommen.

In Kapitel 4 testeten wir empirisch die Idee, dass die Evolution der Gebärmutter und Superfetation ein Leben in einer körperlich anspruchsvollen Umgebung (z.B. hohe Wasserfliessgeschwindigkeit) ermöglicht. Dies als Konsequenz einer Reduktion der körperlichen Belastung während der Schwangerschaft und somit verbesserter Beweglichkeit. Falls korrekt, postulierten wir, dass Arten mit einer Gebärmutter und/oder der Fähigkeit zur Superfetation in der Lage sind körperlich anspruchsvollere (Mikro-)Habitate zu nutzen verglichen mit nahverwandten Arten ohne Gebärmutter oder der Fähigkeit zur Superfetation. Um dies zu testen machten wir Gebrauch von visuellen Unterwasserzählungen um die diurnale und ontogenetische Mikrohabitat Selektion von fünf sympatrischen lebendgebärenden Fischarten (Familie Poeciliidae) in costa-ricanischen Flüssen zu studieren. In Übereinstimmung mit unseren Postulaten haben wir signifikante inter-spezifische Unterschiede in der tageszeitlichen Mikrohabitat Selektion beobachtet: Arten mit einer Gebärmutter und der Fähigkeit zur Superfetation wurden in tieferen und schneller-fliessenden Flussbereichen entdeckt, Arten ohne die beiden Adaptationen waren begrenzt in seichten, langsam-fliessenden Bereichen, und Arten mit nur einer Adaptation (d.h. Superfetation) nutzen intermediäre Flussbereiche. Diese inter-spezifische tageszeitliche Mikrohabitat Selektion war am stärksten in reproduzierenden Alttieren, intermediär in noch nicht voll ausgereiften Tieren, und abwesend in Jungtieren, was auf einen Einfluss der Ontogenie auf die artspezifische Mikrohabitat Selektion schliessen lässt. Letztendlich, während der Nacht, versammelten sich alle Fische unabhängig von der Art oder Alter in den seichten, langsam-fliessenden Flussbereichen um sich auf dem Flussgrund zu erholen (d.h. um zu schlafen). Unsere Resultate lassen vermuten, dass die Gebärmutter und Superfetation bislang unerkannte Fortpflanzungsmerkmale darstellen, welche helfen Unterschiede in den ontogenetischen und diurnalen Mikrohabitat Präferenzen zwischen sympatrischen lebendgebärenden Fischarten zu verstehen.

In Kapitel 5 erforschten wir die Konsequenzen einer Trematoden Infektion (Schwarzpunkt Krankheit, BSD) für die life-history und Verhalten (Dreistigkeitstests im Feld) der plazental lebendgebärenden Fischart P. retropinna von Costa Rica. Wir postulierten, dass die intime plazentale Verknüpfung zwischen Mutter und Fetus ein Risiko birgt, weil eine Parasiteninfektion möglicherweise negative Konsequenzen für die fetale Entwicklung hat. Zum Beispiel kann eine mütterliche Parasiteninfektion die fetale Entwicklung über zwei nicht gegenseitig ausschliessenden Wege beeinflussen: (i) direkt, über die Infektion der sich entwickelnden Embryonen durch Parasiten, die die plazentale Barriere überqueren können, und (ii) indirekt, durch die Modifikation der mütterlichen Physiologie oder des Metabolismus, sodass die fetale Entwicklung beeinträchtigt wird. Wir zeigten beträchtliche Vari- 
ation in der Parasiteninfektion zwischen einzelnen Weibchen innerhalb einer Population, die mit der Grösse und Kondition (ausgedrückt als Köperfett) des mütterlichen Körpers korrelierte. Zudem fanden wir, dass stark parasitierte Weibchen kleinere und schlechter-konditionierte Nachkommen produzieren, wahrscheinlich, weil eine kostspielige Immunreaktion während der Schwangerschaft die Energie limitiert, die verfügbar ist um (i) die sich entwickelnden Embryonen zu ernähren, oder (ii) eine funktionsfähige Gebärmutter zu bilden (indirekter Effekt auf die Nährstoffversorgung der Embryonen). Jedoch hatte die Infektionsrate keinen Effekt auf das individuelle Dreistigkeitsverhalten im Feld. Unsere Resultate zeigen, dass eine Parasiteninfektion in lebendgebärenden Fischen zu einer reduzierten embryonalen Nährstoffversorgung während der Schwangerschaft, und folglich zu kleineren, geringwertigeren Nachkommen mit potentiell negativen Implikationen für deren Fitness führt.

In Kapitel 6 führten wir ein 7-Wochen Laborexperiment durch um die Auswirkungen von mütterlichem Nahrungsmangel während der Schwangerschaft auf die Grösse und Qualität (d.h. Körperfett) der Nachkommen bei der Geburt, sowie Wachstum und LokomotionsFähigkeit nach der Geburt in der plazental lebendgebärenden Fischart Phalloptychus januarius zu studieren. Wir haben gezeigt, dass mütterlicher Nahrungsmangel während der Schwangerschaft das mütterliche Körper-Nassgewicht während des Experiments reduziert, was folglich zu einer reduzierten Nährstoffinvestition in die Grösse und Körperfett der $\mathrm{F}_{1^{-}}$ Nachkommen führt. Dies kann schliesslich nur über eine längere Zeitspanne kompensiert werden (d.h. wenn ausgewachsen). Obwohl dies keine negativen Auswirkungen auf die Schnell-Start Fähigkeit der Nachkommen bei der Geburt hat, hat es negative Implikationen für die postnatale Entwicklung der Lokomotions-Fähigkeit während der Nahrungsaufnahme, und somit Körperkondition und möglicherweise Fitness nach der Geburt. Diese negativen Effekte des mütterlichen Nahrungsmangels auf die Grösse kommt in der $\mathrm{F}_{2^{-}}$ Generation immer noch vor, aber ist eine Woche nach Geburt verschwunden. Dieses Kapitel legt nahe, dass das Besitzen einer Gebärmutter eine maladaptive Strategie in einer Umwelt mit fluktuierenden Ressourcen ist, weil eine plötzliche Reduktion im Nährstoffangebot während der Schwangerschaft die Produktion von kleineren Nachkommen mit einer schlechteren Lokomotions-Fähigkeit in den frühen Lebensstadien verursacht.

Schließlich ordne ich in Kapitel 7 die Ergebnisse dieser Dissertation in den wissenschaftlichen Kontext ein. Zuerst formuliere ich erneut die verschiedenen Hypothesen zur Evolution der Gebärmutter mit den zurzeit vorhandenen Evidenzen. Zweitens, da Superfetation häufig in der Familie Poeciliidae beobachtet wird und eine Co-Evolution zwischen der Gebärmutter und Superfetation postuliert wird, entwarf ich ein R-package um die potentiellen Vorteile von Superfetation, sowie von beiden, Gebärmutter and Superfetation, zu simulieren und graphisch zu illustrieren. Die Simulation zeigt, dass die CoEvolution zwischen der Gebärmutter und Superfetation durch deren additiven Effekte auf die körperlichen Belastung während der Schwangerschaft wahrscheinlich begünstigt wird. Drittens diskutiere ich die potentiellen Nachteile einer Gebärmutter unter verschiedenen 
adversen Umweltbedingungen. Ich komme zu dem Schluss, dass obwohl der Besitz einer Gebärmutter einen adaptiven Vorteil in einer körperlich anspruchsvollen Umgebung aufgrund einer verbesserten Beweglichkeit hat, die intime plazentale Verknüpfung zwischen Mutter und Fetus auch ein Risiko birgt: wenn eine Mutter adversen Umweltbedingungen (z.B. Unterernährung, Parasiteninfektion) ausgesetzt ist, hat dies möglicherweise negative Konsequenzen für die fetale Entwicklung. Zum Schluss präsentiere ich einen Ausblick auf zukünftige Forschung indem ich die Schritte und Herausforderungen thematisiere, die notwendig sind um das Wissen über die Ursachen und Konsequenzen der GebärmutterEvolution zu vertiefen. 


\section{Acknowledgements}

$\mathrm{A} \mathrm{PhD}$ project is clearly a team work. Therefore, the research presented in this thesis could not have been done without the help and dedication of many people. Here, I would like to devote a few words to thank all these people. Also, I would like to apologize to and thank all those who I might have forgotten.

First of all, I want to express my deep gratitude to my daily supervisor Bart. Thank you for believing in me and for giving me the opportunity to perform research with you. You always took the time to address my questions and your insightful suggestions and advice helped me to develop as a scientist. The past four years have been intense at times, but your enthusiasm for live-bearing fish made it easy for me to greatly profit from your knowledge. Not only have I learned a lot, but you also convinced me that live-bearing fish are very cool and unique model systems. I have very enjoyed the time with you at Wageningen University ( WUR), but our yearly field trips to Costa Rica were clearly the highlight. Together with Andrew we developed to a very effective and successful research unit. I will never forget the amazing moments wading through the jungle streams of Costa Rica hunting for live-bearing fish, reptiles, spiders, and amphibians. Whether we were enjoying a refreshing batido in Buenos Aires, struggling through the dirty (probably toxic) Rio Sucio, or snorkeling in the crystal-clear Rio Gamba, it was always an incredible adventure. Also, I would like to express my deep gratitude to my promotor Johan. Thank you for giving me the opportunity to work at the Experimental Zoology Group (EZO). I greatly appreciated your guidance, suggestions, and advice that have very much improved my work. Despite your full agenda, you always tried to be available for an interesting discussion and you always appreciated my statistical input.

My sincere thanks also go to our collaborators from other universities. From the University of Hull (UK), I would like to thank Andrew. Thank you very much for all your input on our research projects and for the amazing time with you in Costa Rica. I was always impressed by your very fast replies and your incredibly detailed comments and suggestions. I wish you all the best and good success for the future. I hope you can continue working with live-bearing fish. Also, I would like to thank David from the University of California Riverside (USA). I am amazed by your knowledge and I greatly appreciated your feedback on our manuscripts. 
Many thanks go, of course, also to the staff of EZO. Annemarie, you are the best secretary in can think of. You are the reason why I never had problems with my contract, reimbursements, or any other administrative issues. You always take care of your 'children', which gives working at EZO the feeling of being in a family. Henk and Leonardo, thank you very much for providing IT support. Your technical knowledge is essential for the group and has also helped me throughout my project. Remco, thank you very much for building experimental set ups. I just had to show you a sketch on paper and the realization arrived in my office a few days later. Karen, I always enjoyed spending time with you in the lab. Thank you very much for your support and help during the fish dissections.

Dear Myrthe and Pim, my paranymphs, thank you for all the support both in work and in personal matters. It is a honor for me that you are my paranymphs (despite Amsterdam vs. Rotterdam ;-)). Myrthe, you are such a great person and the best office mate I can think of. I have very much enjoyed the time with you in Costa Rica, where we got to know each other better (e.g. by wading through the dirty Rio Sucio). I was always happy that you have worked on a similar project as me, so that we were able to help each other. Thank you very much for all the great moments, discussions, and the amazing parties at your place in Amsterdam (maybe at some point even in your own house). I wish you and Joeri all the best and good success for the future. Pim, you are such an incredibly helpful person, it was always a pleasure for me to give you statistical advice. You work on so many projects simultaneously, but still, you are always available if somebody needs your help, support, or just an after-work beer. I very much appreciate that, and I am sure the complete group too. You were clearly the main reason why all the PhD events were so amazing. From all the funny memories I have, you were either the trigger or at least a contributor. Thank you very much for all the great moments and I hope we can repeat our pub crawls through Arnhem. I wish you all the best and good success for the future.

I am also grateful to all assistant professors, researchers, and teachers of EZO for their input and comments during my lunch break presentations and coffee breaks. Special thanks to Martin, Florian, Sander G., and Sander K., I have greatly appreciated your feedback. I hope I could have given back something by providing statistical support. Anneke, Ellen, Guillermo, and Steffen welcome in the group! Many thanks also to the amazing staff of Carus, Emily, Menno, Sander, Truus, and Wian for taking care of our live-bearing fish and building experimental set ups.

During my project, I had the opportunity to supervise and guide my students Job and Judith. Thank you very much for your support. It was a pleasure for me to supervise you and I learned a lot while working with you. I wish you all the best!

I would also like to say special thanks to all the other wonderful people that I have met 
during the last four years at Zodiac: my past and current office mates (Diego, Henri, Mike, Myrthe, and Tiffany) who were always available for interesting discussions and who made the working in the live-bearing fish office awesome; my great colleagues from the offices next door (Antoine, Cees Megatron, Gauthier, Julian, Lana, Noraly, Pim, Pulkit, Uroš, and Wouter), as well as my colleagues from the Cell Biology and Immunology Group (Adrià, Annelieke, Carmen, Esther, Jules, Julia, Mark, Marloes, Mirelle, Mojtaba, Olaf, Paulina, Sem, and Sylvia). Special thanks to Annelieke, Esther, and Pim who have helped making my shoulder great again. Because of all these wonderful people, the last four years consisted not only of work, but also of a lot of fun. Thank you very much for organizing all kind of events, including PhD-weekends, Friday afternoon drinks, dinners, and many other parties. You made the work enjoyable and I will never forget all the fun we had on all these occasions. I wish you all the best and good success for the future and I hope that we will continue our contact.

My life in the Netherlands (without any vertical landscape variation) would not have been satisfying without the support team Arnhem. Julian, I was very happy to hear that I am not the only one from EZO that lives in Arnhem. You have not only introduced me to Arnhem, but also to your friends and family, which has enormously facilitated my establishment in the Netherlands. Annika, Iris, Julian, Murat, Roel, and Sophie, thank you so much for all the awesome BBQ's, home parties, Beugel hangovers, and drinks in probably every other bar that exists in Arnhem. I will never forget the great time with you and I am sure we will keep in touch.

Last but not least, I would like to thank my family and friends. Liebe Mutter, Vater, und Neni. Ihr habt immer an mich geglaubt, wart immer für mich da, und habt mich bei allem unterstützt. Ich will mich ganz herzlich für eure Wärme und Liebe bedanken und hoffe dass ich euch mit meinem Doktor Titel stolz machen kann. Petri Heil und Waidmanns Heil, ich wünsche uns und unser Jagdgruppe viele weitere Fische, Hirsche, Rehe, Gämse, Wildschweine, und eine tolle Zeit auf unserem Maiensäss. Liebe Gotta Manuela, Götti Kläpf und Silvio, ich danke euch ganz herzlich für all eure Unterstützung in den vergangenen Jahren. Caroline, ma petite chérie, our relationship has started at the same time as my $\mathrm{PhD}$. With ongoing project, our physical distance has increased from 0 to $10,000 \mathrm{~km}$, but our feelings for one another remained the same. I always knew that you are too perfect to care about the distance (but do not take that as a permission to go even further away...). I would not have survived the last four years without your support. Thank you very much for all your love and I hope that we will spend many more wonderful years together.

Detlef und Slavica, vielen herzlichen dank für eure Unterstützung und die alljährliche, wunderschöne Zeit in Bocke. Ich habe dies immer sehr geschätzt und es ist mir immer wieder ein Vergnügen. Armon, Crazy, Dirty Harry, Dom, Gian, Halb Meter, Lettla, Luki, Lumpi, Marc Power Tyron, Mauro, Noni, Pipo, Schaller, Thomas, 
Tschingg, Urs, sowie viele weitere Mitglieder der Knabengesellschaft und Bürger des Weissen Kreuzes Andeer. Dank euch viel es mir leicht während meinen Ferien in der Heimat abzuschalten. Nach einem Wochendende mit euch (und den dazugehörigen Knäll) fühlte ich mich wie 'neu geboren'. Hauptsach as knallt... Ich danke euch ganz herzlich für eure Unterstützung, all das Bier, Corretto Grappa, und Enzian. 


\section{Personalia}

\section{Curriculum vitae}

Andres Hagmayer was born on the $14^{\text {th }}$ of June 1991 in Chur (Switzerland). He grew up in the small village Andeer (Switzerland) in the middle of the Swiss Alps. By spending a lot of time in nature, he got fascinated by biological processes already at a young age. He decided to deepen his knowledge in biology during the gymnasium programme at the Kantonsschule in Chur (Switzerland), after which he received the highest award for an gymnasium thesis at the national contest 'Schweizer Jugend

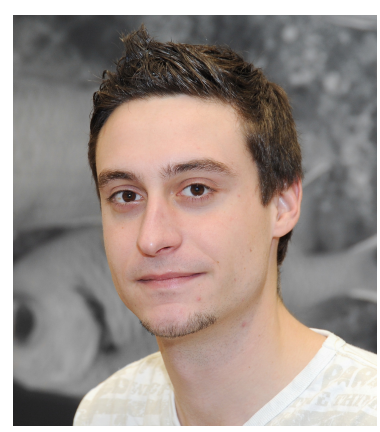
Forscht'.

After a year as a soldier in the Swiss army, he enrolled in the Biology B.Sc. programme at the the University of Zurich (Switzerland), where he obtained his B.Sc. in 2014. During his B.Sc. studies, he became specifically interested in evolutionary biology. Driven by this interest, Andres continued in 2014 with the M.Sc. programme Systematics and Evolution at the the University of Zurich (Switzerland). He specialized in ecological genetics of metabolism by studying a population of snow voles (Chionomys nivalis) in the Swiss Alps. His M.Sc. thesis entitled 'Selective forces and evolutionary potential of metabolism in a wild population of snow voles (Chionomys nivalis)' (supervised by Dr Erik Postma and Dr Timothée Bonnet) was awarded with the highest grade, and later published in a revised form as a peer-reviewed paper in the Journal of Evolutionary Biology.

For his PhD thesis entitled 'Benefits and drawbacks of the placenta in live-bearing fish' (supervised by Dr Bart J.A. Pollux and Prof. Dr Johan L. van Leeuwen) at the Experimental Zoology Group (EZO), Wageningen University \& Research, Wageningen (The Netherlands), Andres focused from 2016 onwards on life-history evolution in placental livebearing fish. The aim of this project, which was funded by the Netherlands Organization for Scientific Research (NWO), was to understand the obscure origin and elaboration of the placenta. The project took place in close collaboration with Dr Andrew I. Furness (University of Hull, UK), and included extensive ecological field work in Costa Rica for one month per year. He finished his thesis within four years, whereas all research chapters of the thesis are published in, or submitted to, peer-reviewed renowned (Q1) journals. 


\section{List of publications}

\section{Peer-reviewed scientific publications}

1. Furness, A. I., Hagmayer, A., and Pollux, B. J. A. (2020). Size-dependent male mating tactics and their morphological correlates in Poecilia gillii. Biological Journal of the Linnean Society, pages 1-18

2. Hagmayer, A., Furness, A. I., and Pollux, B. J. A. (2020b). Parasite infestation influences life history but not boldness behavior in placental live-bearing fish. Oecologia, 194:635-648

3. Dekker, M. L., Hagmayer, A., Leon-Kloosterziel, K. M., Furness, A. I., and Pollux, B. J. A. (2020). High degree of multiple paternity and reproductive skew in the highly fecund live-bearing fish Poecilia gillii (Family Poeciliidae). Frontiers in Ecology and Evolution, 8:1-14

4. Hagmayer, A., Camenisch, G., Canale, C., Postma, E., and Bonnet, T. (2020a). Limited mass-independent individual variation in resting metabolic rate in a wild population of snow voles (Chionomys nivalis). Journal of Evolutionary Biology, 33(5):608-618

5. Hagmayer, A., Furness, A. I., Reznick, D. N., Dekker, M. L., and Pollux, B. J. A. (2020c). Predation risk shapes the degree of placentation in natural populations of live-bearing fish. Ecology Letters, 23(5):831-840

6. Hagmayer, A., Furness, A. I., Reznick, D. N., and Pollux, B. J. A. (2018). Maternal size and body condition predict the amount of post-fertilization maternal provisioning in matrotrophic fish. Ecology and Evolution, 8(24):12386-12396

\section{Conferences and seminars}

1. Pollux, B.J.A., and Hagmayer, A. The evolution of placentas in the live-bearing fish Poeciliopsis retropinna from Costa Rica. Virtual Fish Lab Meeting, 30 October 2020, Department of Biology, University of Oklahoma, USA. (talk)

2. Hagmayer, A., Furness, A.I., Reznick, D.N., and Pollux, B.J.A. Predation risk drives the evolution of the placenta in the livebearing placental fish Poeciliopsis retropinna. The 2019 Congress of the European Society for Evolutionary Biology (ESEB), 19-22 August 2019, Turku, Finland. (talk)

3. Hagmayer, A. Predation risk drives the evolution of the placenta in natural populations of live bearing fish. Masterclass at Wageningen University with Prof. David Reznick, 27 May 2019, Wageningen, The Netherlands. (talk)

4. Hagmayer, A., and Pollux, B.J.A. Placental fish as a model system to study prenatal stress effects on offspring development. Masterclass at the University of Amsterdam with Prof. Tracy Bale, 6 February 2019, Amsterdam, The Netherlands. (talk)

5. Pollux, B.J.A., Hagmayer, A., and Furness, A.I. Why do placentas evolve in livebearing fishes? 1st NWO life sciences congress (Life2019), 28-29 May 2019, Bunnik, 
The Netherlands. (talk)

6. Hagmayer, A., Furness, A.I., Reznick, D.N., and Pollux, B.J.A. Sources of placental variation on the micro-evolutionary level. Behavioral Ecology Seminar, 30 October 2018, Wageningen, The Netherlands. (talk)

7. Guernsey, M., Hagmayer, A., Reznick, D.N., Pollux, B.J.A., and Baker, J. Convergence and divergence: the story of placenta evolution as told by Poeciliopsis fishes. International Federation of Placenta Associations (IFPA), 21-24 September 2018, Tokyo, Japan. (talk)

8. Hagmayer, A., Furness, A.I., Reznick, D.N., and Pollux, B.J.A. Maternal effects in a placental live-bearing fish. Netherlands Annual Ecology Meeting (NAEM), 13-14 February 2018, Lunteren, The Netherlands. (talk)

9. Hagmayer, A., Furness, A.I., Reznick, D.N., and Pollux, B.J.A. Maternal effects in a placental live-bearing fish. WIAS Science Day, 5 February 2018, Wageningen, The Netherlands. (talk)

10. Hagmayer, A., Bonnet, T., and Postma, E. Metabolism, body mass and fitness in a wild population of snow voles (Chionomys nivalis). The Swiss conference on organismic biology (Biology15), 12-13 February 2015, Dübendorf, Switzerland. (poster) 


\section{Training and supervision plan}

With the training and education activities listed below Andres Hagmayer has complied with the requirements set by the Graduate School of the Wageningen Institute of Animal Sciences (WIAS) which comprises of a minimum total of 30 ECTS (1 ECTS equals a study load of 28 hours).

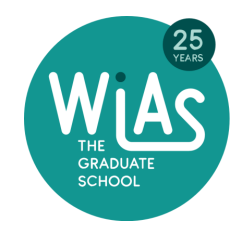

\begin{tabular}{lcc}
\hline Activity & Year & ECTS \\
\hline The basic package & & $\mathbf{1 . 8}$ \\
\hline WIAS Introduction Day, WUR & 2016 & \\
Course on Philosophy of Science and/or Ethics, WUR & 2017 &
\end{tabular}

Disciplinary competences

Laboratory Animal Sciences, WUR 2017

PhD Research Proposal, WUR 2017

Advancing in Statistical Modelling for Evolutionary Biologists and Ecolo- 2017 gists using $\mathrm{R}, U K$

Getting started with ASReml, WUR 2017

Introduction to Bayesian Hierarchical Modelling using R, UK 2018

Applied Bayesian Modelling for Ecologists and Epidemiologists, UK 2020

Professional competences

Reviewing a Scientific Paper, WUR

2017

The Essentials of Scientific Writing and Presenting, WUR 2018

Teaching and Supervising Thesis Students, WUR 2019

The Final Touch, WUR 2019

Efficient Writing Strategies, WUR 2019

WIAS course High Impact Writing in Science, WUR 2019

Adobe InDesign Essential Training, WUR 2020

Presentation skills

4

WIAS Science Day, Wageningen, The Netherlands (oral presentation) 2018

Netherlands Annual Ecology Meeting (NAEM), Lunteren, The Netherlands 2018

(oral presentation)

The 2019 Congress of the European Society for Evolutionary Biology 2019 (ESEB), Turku, Finland (oral presentation)

Evolution 2020 (cancelled due to COVID-19), Cleveland, USA (oral presen- 2020

tation abstract accepted)

Teaching competences 

This work is part of the VIDI grant 'The evolution of the fish placenta: a microevolutionary study of conflict and adaptation' with project number 864.14.008, which is financed by the Netherlands Organisation for Scientific Research (NWO).

Financial support from the Experimental Zoology Group (Wageningen University) for printing this thesis is gratefully acknowledged.

\section{Quotes on the artworks accompanying the chapter title-pages}

Chapter 1: Image modified from:

https://nl.pinterest.com/pin/134334001365295025/

Chapter 2: Photo credit Poeciliopsis retropinna: Andrew Furness; photo credit Poeciliopsis retropinna embryo development: Andres Hagmayer

Chapter 3: Bass image modified from: https://www.mossyoak.com/our-obsession/ blogs/fishing/an-expert-guide-to-winter-bass-fishing; photo credit Poeciliopsis retropinna: Andrew Furness

Chapter 4: Heron image modified from: https://www.alamy.com/stock-photo/ heron-eating-fish.html; photo credit Poeciliopsis retropinna: Andrew Furness

Chapter 5: Photo credit Poeciliopsis retropinna: Andrew Furness

Chapter 6: Photo credit Phallopthychus januarius: Mike Fleuren; Artemia salina image modified from: http://www.kakerlakenparade.de/artemia.html

Chapter 7: Image modified from:

https://nl.pinterest.com/pin/134334001365295025/

\section{Layout and typesetting}

by Andres Hagmayer,

Texmaker, v. 5.0.3

\section{Printing}

by Proefschriftmaken, The Netherlands, on $115 \mathrm{~g}$ silk paper.

Design of artworks accompanying the chapter title-pages by Andres Hagmayer

\section{Design of cover}

by Stefanie van den Herik, Master of Communication \& Information Sciences (CIS), SVDHMEDIA, https://svdhmedia.com/ 

So 